\title{
Contribuições em inferência e modelagem de valores extremos
}

\author{
Eliane Cantinho Pinheiro
}

TESE APRESENTADA

$\mathrm{AO}$

Instituto De MATEMÁTiCA E EstatístiCA

DA

UNIVERSIDAdE DE SÃo PAUlo

PARA

OBTENÇÃO DO TÍTULO

DE

DOUTOR EM CIÊNCIAS

\author{
Programa: Estatística \\ Orientador: Prof ${ }^{\mathrm{a}}$. Dr ${ }^{\mathrm{a}}$. Silvia Lopes de Paula Ferrari
}

Durante o desenvolvimento deste trabalho a autora recebeu auxílio financeiro da CAPES e do CNPQ.

São Paulo, 17 de junho de 2014 
"O que é impossível aos homens, é possível a Deus."

Jesus

"Tudo o que fizeres, faze-o na medida do teu talento, e terás louvado a Deus."

Sto Agostinho

"Eu não vejo graça em outras coisas como eu vejo em cantar."

Elis Regina

Dedico este trabalho a todos os que me instruíram na Verdade, a todos os que me indicaram boas leituras, a todos os que me incentivaram, a todos os que me fizeram crescer e a todos os que tornaram meu caminho mais suave. 


\section{Agradecimentos}

A Deus pelo dom da vida e pelo dom de amá-la.

Ao meu marido pelo incentivo e parceria.

Aos meus filhos por alegrarem minha vida.

Aos meus pais por sua dedicação.

À minha sogra por seu exemplo de fé.

Ao meu sogro por seu exemplo de estudante perene.

À professora Silvia pelo entusiasmo contagiante e disponibilidade.

À CAPES e ao CNPQ pelo apoio financeiro.

Aos participantes da banca examinadora. 


\section{Resumo}

A teoria do valor extremo é aplicada em áreas de pesquisa tais como hidrologia, estudos de poluição, engenharia de materiais, controle de tráfego e economia. A distribuição valor extremo ou Gumbel é amplamente utilizada na modelagem de valores extremos de fenômenos da natureza e no contexto de análise de sobrevivência para modelar o logaritmo do tempo de vida. A modelagem de valores extremos de fenômenos da natureza tais como velocidade de vento, nível da água de rio ou mar, altura de onda ou umidade é importante em estatística ambiental pois o conhecimento de valores extremos de tais eventos é crucial na prevenção de catátrofes. Ultimamente esta teoria é de particular interesse pois fenômenos extremos da natureza têm sido mais comuns e intensos.

A maioria dos artigos sobre teoria do valor extremo para modelagem de dados considera amostras de tamanho moderado ou grande. A distribuição Gumbel é frequentemente incluída nas análises mas a qualidade do ajuste pode ser pobre em função de presença de ouliers.

Investigamos modelagem estatística de eventos extremos com base na teoria de valores extremos. Consideramos um modelo de regressão valor extremo introduzido por Barreto-Souza \& Vasconcellos (2011). Os autores trataram da questão de corrigir o viés do estimador de máxima verossimilhança para pequenas amostras. Nosso primeiro objetivo é deduzir ajustes para testes de hipótese nesta classe de modelos. Derivamos a estatística da razão de verossimilhanças ajustada de Skovgaard (2001) e cinco ajustes da estatística da razão de verossimilhanças sinalizada, que foram propostos por Barndorff-Nielsen $(1986,1991)$, DiCiccio \& Martin (1993), Skovgaard (1996), Severini (1999) e Fraser et al. (1999). As estatísticas ajustadas são aproximadamente distribuídas como uma distribuição $\chi^{2}$ e normal padrão com alto grau de acurácia. Os termos dos ajustes têm formas compactas simples que podem ser facilmente implementadas em softwares disponíveis. Comparamos a performance do teste da razão de verossimilhanças, do teste da razão de verossimilanças sinalizada e dos testes ajustados obtidos neste trabalho em amostras pequenas. Ilustramos uma aplicação dos testes usuais e suas versões modificadas em conjuntos de dados reais. As distribuições das estatísticas ajustadas são mais próximas das respectivas distribuições limites comparadas com as distribuições das estatísticas usuais quando o tamanho da amostra é relativamente pequeno. Os resultados de simulação indicaram que as estatísticas ajustadas são recomendadas para inferência em modelo de regressão valor extremo quando o tamanho da amostra é moderado ou pequeno.

Parcimônia é importante quando os dados são escassos, mas flexibilidade também é crucial pois um ajuste pobre pode levar a uma conclusão completamente errada. Uma revisão da literatura foi feita para listar as distribuições que são generalizações da distribuição Gumbel. Nosso segundo objetivo é avaliar a parcimônia e flexibilidade destas distribuições. Com este propósito, comparamos tais distribuições através de momentos, coeficientes de assimetria e de curtose e índice da cauda. As famílias mais amplas obtidas 
pela inclusão de parâmetros adicionais, que têm a distribuição Gumbel como caso particular, apresentam assimetria e curtose flexíveis enquanto a distribuição Gumbel apresenta tais características constantes. Dentre estas distribuições, a distribuição valor extremo generalizada é a única com índice da cauda que pode ser qualquer número real positivo enquanto os índices da cauda das outras distribuições são zero. Observamos que algumas generalizações da distribuição Gumbel estudadas na literatura são não identificáveis. Portanto, para estes modelos a interpretação e estimação de parâmetros individuais não é factível. Selecionamos as distribuições identificáveis e as ajustamos a um conjunto de dados simulado e a um conjunto de dados reais de velocidade de vento. Como esperado, tais distribuições se ajustaram bastante bem ao conjunto de dados simulados de uma distribuição Gumbel. A distribuição valor extremo generalizada e a mistura de duas distribuições Gumbel produziram melhores ajustes aos dados do que as outras distribuições na presença não desprezível de observações discrepantes que não podem ser acomodadas pela distribuição Gumbel e, portanto, sugerimos que tais distribuições devem ser utilizadas neste contexto.

Palavras-chave: Ajustes para pequenas amostras; Generalizações da distribuição Gumbel; Generalizações da distribuição valor extremo; Modelos não lineares; Regressão valor extremo; Teste da razão de verossimilhanças; Teste da razão de verossimilhanças sinalizada. 


\section{Abstract}

The extreme value theory is applied in research fields such as hydrology, pollution studies, materials engineering, traffic management, economics and finance. The Gumbel distribution is widely used in statistical modeling of extreme values of a natural process such as rainfall and wind. Also, the Gumbel distribution is important in the context of survival analysis for modeling lifetime in logarithmic scale. The statistical modeling of extreme values of a natural process such as wind or humidity is important in environmental statistics; for example, understanding extreme wind speed is crucial in catastrophe/disaster protection. Lately this is of particular interest as extreme natural phenomena/episodes are more common and intense.

The majority of papers on extreme value theory for modeling extreme data is supported by moderate or large sample sizes. The Gumbel distribution is often considered but the resulting fit may be poor in the presence of ouliers since its skewness and kurtosis are constant.

We deal with statistical modeling of extreme events data based on extreme value theory. We consider a general extreme-value regression model family introduced by Barreto-Souza \& Vasconcellos (2011). The authors addressed the issue of correcting the bias of the maximum likelihood estimators in small samples. Here, our first goal is to derive hypothesis test adjustments in this class of models. We derive Skovgaard's adjusted likelihood ratio statistics (Skovgaard, 2001) and five adjusted signed likelihood ratio statistics, which have been proposed by Barndorff-Nielsen (1986, 1991), DiCiccio \& Martin (1993), Skovgaard (1996), Severini (1999) and Fraser et al. (1999). The adjusted statistics are approximately distributed as $\chi^{2}$ and standard normal with high accuracy. The adjustment terms have simple compact forms which may be easily implemented by readily available software. We compare the finite sample performance of the likelihood ratio test, the signed likelihood ratio test and the adjusted tests obtained in this work. We illustrate the application of the usual tests and their modified versions in real datasets. The adjusted statistics are closer to the respective limiting distribution compared to the usual ones when the sample size is relatively small. Simulation results indicate that the adjusted statistics can be recommended for inference in extreme value regression model with small or moderate sample size.

Parsimony is important when data are scarce, but flexibility is also crucial since a poor fit may lead to a completely wrong conclusion. A literature review was conducted to list distributions which nest the Gumbel distribution. Our second goal is to evaluate their parsimony and flexibility. For this purpose, we compare such distributions regarding moments, skewness, kurtosis and tail index. The larger families obtained by introducing additional parameters, which have Gumbel embedded in, present flexible skewness and kurtosis while the Gumbel distribution skewness and kurtosis are constant. Among these distributions the generalized extreme value is the only one with tail index that can be any positive real number while the tail indeces of the other distributions investigated here are zero. We notice that some generalizations of the Gumbel 
distribution studied in the literature are not indetifiable. Hence, for these models meaningful interpretation and estimation of individual parameters are not feasible. We select the identifiable distributions and fit them to a simulated dataset and to real wind speed data. As expected, such distributions fit the Gumbel simulated data quite well. The generalized extreme value distribution and the two-component extreme value distribution fit the data better than the others in the non-negligible presence of outliers that cannot be accommodated by the Gumbel distribution, and therefore we suggest them to be applied in this context.

Keywords: Generalized extreme-value distributions; Extreme-value regression; Generalized Gumbel distributions; Likelihood ratio test; Nonlinear models; Signed likelihood ratio test; Small-sample adjustments; 


\section{Sumário}

Lista de Figuras

Lista de Tabelas

1 Introdução 1

2 Razão de verossimilhanças $\quad 9$

2.1 Modelo de regressão valor extremo máximo . . . . . . . . . . . . . . . . . . . . . . 9

2.2 Ajuste de Skovgaard - valor extremo máximo . . . . . . . . . . . . . . . 13

2.3 Modelo de regressão valor extremo mínimo . . . . . . . . . . . . . . . . . . 15

2.4 Simulações . . . . . . . . . . . . . . . . . . . . . . . . . . . . . . . . 19

2.4.1 modelo linear com dispersão constante . . . . . . . . . . . . . . . . . . 19

2.4.2 modelo linear com dispersão variável . . . . . . . . . . . . . . . . . . . 21

2.4.3 modelo não linear com dispersão constante . . . . . . . . . . . . . . . . . . 28

2.4.4 modelo não linear com dispersão variável . . . . . . . . . . . . . . . . . . . . 29

2.5 Aplicações . . . . . . . . . . . . . . . . . . . . . . . . . . 31

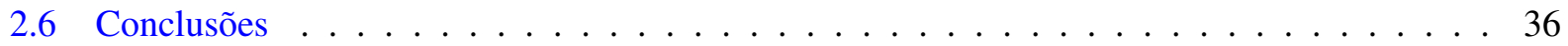

3 Razão de verossimilhanças sinalizada $\quad 41$

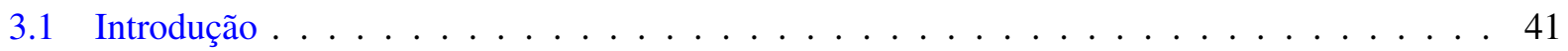

3.2 Ajuste de Barndorff-Nielsen . . . . . . . . . . . . . . . . . . . . 42

3.3 Aproximações para Barndorff-Nielsen . . . . . . . . . . . . . . . . . . . . 44

3.3.1 Ajuste de DiCiccio \& Martin (1993) . . . . . . . . . . . . . . . . . 44

3.3 .2 Ajuste de Skovgaard . . . . . . . . . . . . . . . . . . . . . 47

3.3 .3 Ajuste de Severini . . . . . . . . . . . . . . . . . . . . 48

3.3 .4 Ajuste de Fraser, Reid \& $\mathrm{Wu} \ldots \ldots . . \ldots$. . . . . . . . . . . . . 49

3.4 Simulações . . . . . . . . . . . . . . . . . . . . . . . . . . . . 49

3.4.1 modelo linear com dispersão constante . . . . . . . . . . . . . . . . 50

3.4.2 modelo linear com dispersão variável . . . . . . . . . . . . . . . . . 51

3.4.3 modelo não linear com dispersão constante . . . . . . . . . . . . . . . . . . 53

3.5 Aplicações . . . . . . . . . . . . . . . . . . . . . . 54

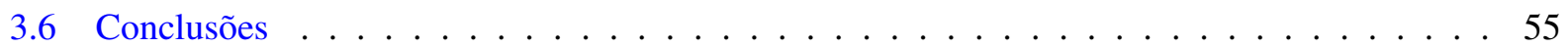


4 Generalizações da distribuição Gumbel $\quad 57$

4.1 Introdução . . . . . . . . . . . . . . . . . . . . . . . . . . 57

4.2 Generalizações da distribuição Gumbel . . . . . . . . . . . . . . . . . . . . 59

4.2 .1 Distribuição valor extremo generalizada . . . . . . . . . . . . . . . . . 59

4.2.2 Distribuição Gumbel exponencializada . . . . . . . . . . . . . . . . . . . . 62

4.2 .3 Distribuição valor extremo transmutada . . . . . . . . . . . . . . . . 64

4.2 .4 Distribuição Kumaraswamy Gumbel . . . . . . . . . . . . . . . . . . . . . . . 67

4.2 .5 Distribuição Gumbel generalizada . . . . . . . . . . . . . . . . . . . . . . 68

4.2.6 Distribuição exponencial-gama com três parâmetros . . . . . . . . . . . . 70

4.2 .7 Distribuição exponencial-gama . . . . . . . . . . . . . . . . . 71

4.2.8 Distribuição logística generalizada tipo IV . . . . . . . . . . . . . . . . . . 73

4.2.9 Distribuição Gumbel exponencializada generalizada . . . . . . . . . . . . . 75

4.2 .10 Distribuição beta Gumbel . . . . . . . . . . . . . . . . . . . . 75

4.2.11 Distribuição Kummer beta generalizada Gumbel . . . . . . . . . . . . . 76

4.2.12 Mistura de dois componentes de distribuição valor extremo máximo . . . . . . . . 77

4.3 Indice da cauda . . . . . . . . . . . . . . . . . . . . . . . 80

4.4 Medidas de qualidade de ajuste . . . . . . . . . . . . . . . . . . . . . . 81

4.5 Aplicações . . . . . . . . . . . . . . . . . . . . . . 84

4.5.1 Amostra simulada de distribuição Gumbel . . . . . . . . . . . . . . . . . . . . 85

4.5.2 Velocidade máxima mensal do vento em West Palm Beach de 1984-2012 . . . . . . 88

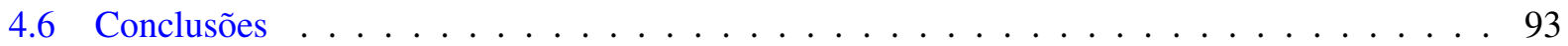

5 Conclusão $\quad 97$

A Escore e Informação - valor extremo máximo 99

B Cálculo de $q$ e $\Upsilon$ - valor extremo máximo $\quad 103$

$\begin{array}{lll}\text { C Cálculo de esperanças das matrizes - valor extremo máximo } & 107\end{array}$

$\begin{array}{ll}\text { D Distribuição das estatísticas } & 113\end{array}$

E Ajustes da razão de verossimilhanças sinalizada $\quad 119$

E.1 Barndorff-Nielsen (1986) . . . . . . . . . . . . . . . . . . . . . . . . . . 119

E.2 DiCiccio \& Martin (1993) . . . . . . . . . . . . . . . . . . . . 122

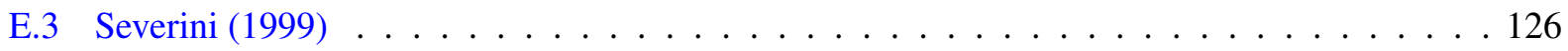

E.4 Fraser, Reid \& Wu (1999) . . . . . . . . . . . . . . . . . . . . . . . . . 127

F Generalizações da distribuição Gumbel 129

F.1 Distribuição valor extremo generalizada . . . . . . . . . . . . . . . . . . . . . . . . . . 129

F.2 Distribuição Gumbel exponencializada . . . . . . . . . . . . . . . . . . . . . 132

F.3 Distribuição valor extremo transmutada . . . . . . . . . . . . . . . . . 132 
F.4 Distribuição valor extremo máximo tipo I ou Gumbel generalizada . . . . . . . . . . . 136

F.5 Distribuição exponencial-gama com três parâmetros . . . . . . . . . . . . . . 137

F.6 Distribuição exponencial-gama . . . . . . . . . . . . . . . . . . . . . . . 139

F.7 Distribuição logística generalizada tipo IV . . . . . . . . . . . . . . . . . . . 139

F.8 Mistura de dois componentes de distribuição valor extremo máximo . . . . . . . . . . . . 142

G Índice da cauda $\quad 143$

G.1 Distribuição valor extremo generalizada . . . . . . . . . . . . . . . . . . . . . 143

G.2 Distribuiç̧ão Gumbel exponencializada . . . . . . . . . . . . . . . . . . . . . . . 144

G.3 Distribuição valor extremo transmutada . . . . . . . . . . . . . . . . . . 144

G.4 Distribuição valor extremo máximo tipo I ou Gumbel generalizada com três parâmetros . . 145

G.5 Distribuição exponencial-gama com três parâmetros ～. . . . . . . . . . . . . . . . . 145

G.6 Distribuição logística generalizada tipo IV . . . . . . . . . . . . . . . . . . . . 145

G.7 Mistura de dois componentes de distribuição valor extremo máximo . . . . . . . . . . . 145

$\begin{array}{ll}\text { Referências Bibliográficas } & 149\end{array}$ 


\section{Lista de Figuras}

2.1 Discrepâncias relativas de quantis; modelo $1 . \ldots \ldots \ldots \ldots \ldots$. . . . . . . . 21

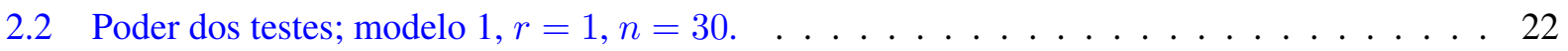

2.3 Discrepâncias relativas de quantis; modelo 2 , localização. . . . . . . . . . . . . . . . . . 24

2.4 Discrepâncias relativas de quantis; modelo 2.1, dispersão . . . . . . . . . . . . . . . . 26

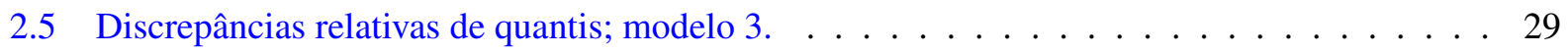

2.6 Discrepâncias relativas de quantis; modelo 4, dispersão. . . . . . . . . . . . . . . . . . 30

2.7 Intervalos de confiança com coeficiente de confiança de $95 \%$; comprimentos dos intervalos entre parênteses - trigo. . . . . . . . . . . . . . . . . 33

3.1 Discrepâncias relativas de valor-p, modelo $1 . \ldots \ldots \ldots$. . . . . . . . . 51

3.2 Discrepâncias relativas de valor-p; modelo 2 , localização. . . . . . . . . . . . . . . . 53

3.3 Discrepâncias relativas de valor-p; modelo $3 . \ldots \ldots \ldots$. . . . . . . . . . . 54

4.1 Função densidade de probabilidade de distribuição $\operatorname{Gumbel}(\mu, \sigma)$. . . . . . . . . . . . 58

4.2 Função densidade de probabilidade de distribuição valor extremo generalizada $\operatorname{GEV}(0,1, \alpha) . \quad 61$

4.3 Gráficos dos coeficientes de assimetria e de curtose da distribuição valor extremo generali-

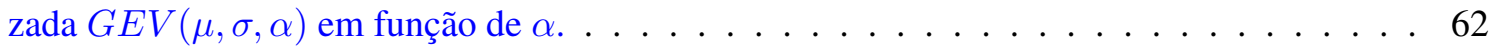

4.4 Função densidade de probabilidade da distribuição Gumbel exponencializada $\operatorname{EGu}(0,1, \alpha) . \quad 63$

4.5 Gráficos dos coeficientes de assimetria e de curtose da distribuição Gumbel exponencializada $E G u(\mu, \sigma, \alpha)$ em função de $\alpha \ldots \ldots \ldots \ldots$. . . . . . . . . . . . . 64

4.6 Função densidade de probabilidade da distribuição $\operatorname{TEV}(0,1, \alpha) \ldots \ldots$. . . . . . . . . 66

4.7 Gráficos dos coeficientes de assimetria e de curtose da distribuição valor extremo transmu-

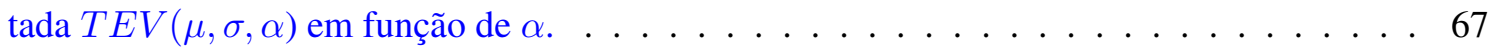

4.8 Função densidade de probabilidade de distribuição valor extremo máximo tipo I ou Gumbel generalizada com três parâmetros $G G u 3(0,1, \alpha)$. . . . . . . . . . . . . . . . . . . . 69

4.9 Gráficos dos coeficientes de assimetria e de curtose da distribuição Gumbel generalizada com três parâmetros $G G u 3(\mu, \sigma, \alpha)$ em função de $\alpha . \ldots$. . . . . . . . . . . . . . 70

4.10 Função densidade de probabilidade da distribuição $\operatorname{Exp} \operatorname{Gama3}(0,1, \alpha)$. . . . . . . . 71

4.11 Gráficos dos coeficientes de assimetria e de curtose da distribuição exponencial-gama com três parâmetros ExpGama3 $(\mu, \sigma, \alpha)$ em função de $\alpha \ldots \ldots \ldots \ldots$. . . . . . . . . 72

4.12 Função densidade de probabilidade da distribuição $\operatorname{GLIV}(0,1, \alpha, \beta) \ldots \ldots \ldots$ 
4.13 Gráficos dos coeficientes de assimetria e de curtose da distribuição logística generalizada tipo IV $G L I V(\mu, \sigma, \alpha, \beta)$ em função de $\alpha$ e $\beta \ldots \ldots \ldots \ldots \ldots$

4.14 Função densidade de probabilidade da distribição $\operatorname{TCEV}(0,1,10,5, \alpha)$. . . . . . . . 78

4.15 Gráficos dos coeficientes de assimetria e de curtose da mistura de dois componentes de distribuição Gumbel TCEV $(0,1,10,5, \alpha)$. . . . . . . . . . . . . . . . . . . . . 79

4.16 Gráfico de observação versus índice e boxplot ajustado de amostra simulada de distribuição

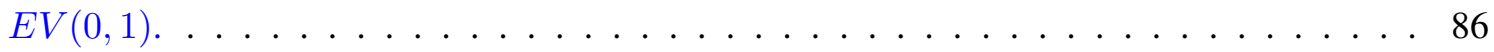

4.17 Histograma de amostra simulada de distribuição $E V(0,1)$ e densidades ajustadas. . . . . . 87

4.18 QQplots - amostra simulada de distribuição $E V(0,1) ， \ldots \ldots$. . . . . . . . . . . . . . . 89

4.19 Logaritmo da verossimilhança perfilada - amostra simulada de distribuição $E V(0,1)$. . . . 90

4.20 Diagrama de dispersão e boxplot ajustado da velocidade máxima mensal do vento (milhas/hora) em West Palm Beach/Flórida de 1984 a 2012. . . . . . . . . . . . . . . . . . . . 91

4.21 Histograma e densidades ajustadas da velocidade máxima mensal do vento (milhas/hora)

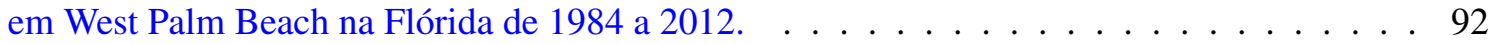

4.22 QQplots - velocidade máxima mensal do vento (milhas/hora) em West Palm Beach na Flórida de 1984 a $2012 \ldots \ldots \ldots$. . . . . . . . . . . . . . . . . . . . . . 94

4.23 Logaritmo da verossimilhança perfilada - velocidade máxima mensal do vento (milhas/hora) em West Palm Beach na Flórida de 1984 a 2012 . . . . . . . . . . . . . . . . . . . . . 95 


\section{Lista de Tabelas}

2.1 Taxas de rejeição nula $(\%) ;$ modelo $1 \ldots \ldots \ldots \ldots \ldots$

2.2 Taxas de rejeição não nula $(\%)$; modelo $1, n=30, \alpha=10 \% \ldots \ldots \ldots 22$

2.3 Taxas de rejeição nula $(\%)$; modelo 2 , localização . . . . . . . . . . . . . . . . 23

2.4 Taxas de rejeição não nula (\%); modelo 2 , localização, $n=40, \alpha=10 \% \ldots \ldots$

2.5 Taxas de rejeição nula $(\%)$; modelo 2.1 , dispersão . . . . . . . . . . . . . . . . 25

2.6 Taxas de rejeição não nula (\%); modelo 2.1 , dispersão, $n=50, \alpha=10 \%$. . . . . . . . 27

2.7 Taxas de rejeição nula $(\%) ;$ modelo $3 \ldots \ldots \ldots \ldots$. . . . . . . . . . 28

2.8 Taxas de rejeição não nula $(\%)$; modelo $3, n=15, \alpha=10 \% \ldots \ldots \ldots$

2.9 Taxas de rejeição nula $(\%)$; modelo 4 , dispersão . . . . . . . . . . . . . . . . . . 30

2.10 Taxas de rejeição não nula (\%); modelo 4 , dispersão, $n=40, \alpha=10 \%$. . . . . . . . . 31

2.11 Peso de hastes de trigo seco. . . . . . . . . . . . . . . . . . 31

2.12 Estimativas dos parâmetros e erros padrão: modelo I - trigo . . . . . . . . . . . . . . 32

2.13 Intervalos de confiança com coeficiente de confiança de $95 \%$ - trigo . . . . . . . . . 32

2.14 Resultados dos testes da hipótese nula $\mathcal{H}_{0}: \beta_{2}=\beta_{c}$ contra $\mathcal{H}_{1}: \beta_{2} \neq \beta_{c}$-trigo . . . . . 34

2.15 Nível do mar em Fremantle, Austrália. . . . . . . . . . . . . . . . . . . 37

2.16 Resultados dos testes da hipótese nula $\mathcal{H}_{0}: \gamma_{3}=0$ contra $\mathcal{H}_{1}: \gamma_{3} \neq 0$ - 'modelo I' - fremantle 38

2.17 Resultados dos testes da hipótese nula $\mathcal{H}_{0}: \gamma_{2}=0$ contra $\mathcal{H}_{1}: \gamma_{2} \neq 0$ - 'modelo II' -

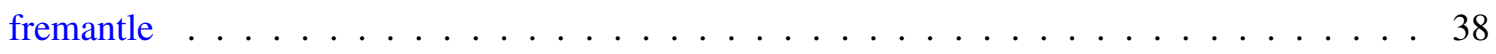

2.18 Resultados dos testes da hipótese nula $\mathcal{H}_{0}: \beta_{3}=0$ contra $\mathcal{H}_{1}: \beta_{3} \neq 0$ - 'modelo III' -

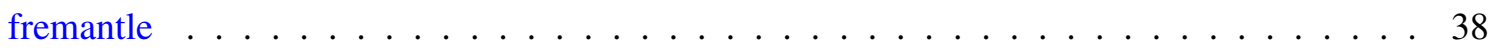

2.19 Resultados dos testes da hipótese nula $\mathcal{H}_{0}: \gamma_{1}=0$ contra $\mathcal{H}_{1}: \gamma_{1} \neq 0$ - 'modelo IV' -

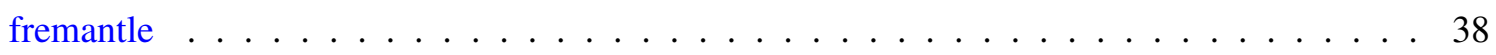

2.20 Resultados dos testes de hipótese - fremantle . . . . . . . . . . . . . . . 38

2.21 Resultado do teste da hipótese nula $\mathcal{H}_{0}: \beta_{1}=0$ contra $\mathcal{H}_{1}: \beta_{1} \neq 0$ - decatlo . . . . . 39

3.1 Taxas de rejeição nula $(\%) ;$ modelo $1 \ldots \ldots \ldots \ldots \ldots \ldots$

3.2 Taxas de rejeição não nula $(\%)$; modelo $1, n=20, \alpha=10 \% \ldots \ldots$. . . . . . . 52

3.3 Taxas de rejeição nula $(\%)$; modelo 2 , localização . . . . . . . . . . . . . . . . . 52

3.4 Taxas de rejeição não nula (\%); modelo 2 , localização, $n=40, \alpha=10 \% \ldots 53$

3.5 Taxas de rejeição nula $(\%)$ modelo $3 \ldots \ldots \ldots \ldots$. . . . . . . . . . . 54

3.6 Taxas de rejeição não nula (\%); modelo 3, localização, $n=15, \alpha=10 \% \ldots \ldots 5$

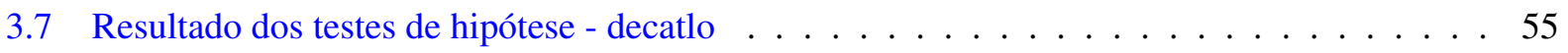


4.1 Diagrama das generalizações da distribuição Gumbel . . . . . . . . . . . . . . . 80

4.2 Índice da cauda de distribuições . . . . . . . . . . . . . . . . . . 81

4.3 Características das generalizações da distribuição Gumbel . . . . . . . . . . . . 85

4.4 Estimativas dos parâmetros, erros padrão entre parênteses e quantis; Amostra simulada de

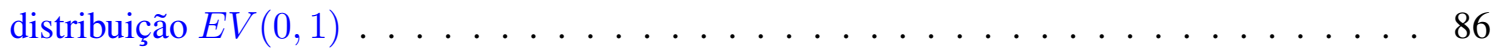

4.5 Bondade de ajuste; Amostra simulada de distribuição $E V(0,1) \ldots \ldots$. . . . . . . . 88

4.6 Estimativas dos parâmetros e erros padrão (entre parênteses); velocidade máxima mensal do vento - West Palm Beach - 1984 a 2012 . . . . . . . . . . . . . . . . . . . . . . . . . 91

4.7 Bondade do ajuste; velocidade máxima mensal do vento - West Palm Beach - 1984 a 2012 . 93 


\section{Capítulo 1}

\section{Introdução}

Os primeiros problemas relacionados a valores extremos surgem das inundações. Sua importância econômica era clara desde que surgiu a economia agrária, exclusivamente baseada no fluxo de água e hidrovias, que eram o principal sistema de comunicação. Tal importância cresceu na economia industrial com a construção de usinas hidrelétricas e reservatórios para irrigação e luta contra a erosão. Além disso, uma previsão adequada de futuras inundações pode preservar vidas e propriedades. A importância social do controle do fluxo de água incentivou o estudo deste tema.

A natureza estatística deste problema fez com que os procedimentos empíricos usados inicialmente fossem substituídos por métodos derivados da teoria dos valores extremos.

A teoria dos valores extremos tem atraído a atenção de muitos cientistas que trabalham em diferentes áreas. Problemas relacionados com valores extremos existem em astronomia, engenharia naval, engenharia eólica, engenharia civil, metalurgia, geologia, oceanografia, meteorologia, predição de tráfego em telecomunicações, estatística populacional, indústria de seguros, avaliação de risco nos mercados financeiros, gestão estratégica, processamento de dados biomédicos, nutrição, etc. Em Kotz \& Nadajarah (2000) encontram-se algumas aplicações como, por exemplo, terremotos, corrida de cavalos, tempestades, filas de supermercados, correntes marítimas, velocidade do vento e recordes em corridas. Recentemente, desastres naturais como o furacão Katrina, o terremoto no Japão, os deslizamentos de terra e as inundações devidas à forte chuva na Austrália, Paquistão e Brasil, além de catástrofes causadas pelo próprio ser humano como vazamentos de óleo ou crises financeiras ressaltaram a necessidade de acurácia nas previsões destes fenômenos complexos.

O objetivo da teoria estatística do valor extremo é analisar valores extremos observados e prever possíveis valores ainda mais extremos, é fazer inferência para eventos cujas probabilidades são menores do que a probabilidade de qualquer evento observado anteriormente. Fenômenos em que a probabilidade de um valor extremo é relativamente alta são caracterizados por distribuições com caudas pesadas. Neste sentido podemos dizer que a teoria estatística do valor extremo difere da teoria estatística clássica que prioriza os momentos centrais, médias e a distribuição normal, que tem caudas leves. Isto exige o uso de modelos particulares, baseados na matemática de variação regular, que permite uma extrapolação adequada, mas também implica que o uso de amostra pequena é inevitável em muitas situações, porque a quantidade de dados relevantes para inferência na cauda da distribuição pode ser limitada, mesmo quando grandes quantidades de dados estão disponíveis para inferência na parte central da distribuição. Em muitas aplicações a inferência para modelos do valor extremo é baseada em procedimentos padrão de primeira ordem baseados 
em verossimilhança sendo natural perguntar se aprimoramentos são necessários.

$\mathrm{Na}$ ausência de diretrizes empíricas ou físicas para formular uma regra de extrapolação, modelos são deduzidos da teoria assintótica. No caso mais simples isto funciona como segue. Sejam $X_{1}, X_{2}, \ldots$ uma sequência de variáveis aleatórias independentes com mesma distribuição $F$ e seja

$$
M_{n}=\max \left\{X_{1}, X_{2}, \ldots, X_{n}\right\}
$$

o valor máximo de $n$ observações. Na prática o comportamento de $X_{i}$ não é conhecido, logo não é possível calcular exatamente o comportamento de $M_{n}$. Contudo, sob condições adequadas, deduz-se o comportamento aproximado de $M_{n}$ para valores grandes de $n$, a partir da teoria do limite quando $n \rightarrow \infty$, gerando uma família de modelos que podem ser calibrados pelos dados observados de $M_{n}$. Esta abordagem é chamada de paradigma do valor extremo, pois envolve um princípio para extrapolação de modelos baseado no uso de limites como aproximação de níveis finitos. É similar à prática de aproximar a distribuição da média amostral pela distribuição normal, com base no Teorema Limite Central.

Se a distribuição exata da estatística $X_{i}$ é conhecida, a distribuição correspondente de $M_{n}$ pode ser calculada exatamente para todos os valores de $n$ :

$$
\begin{aligned}
\operatorname{Pr}\left\{M_{n} \leq x\right\} & =\operatorname{Pr}\left\{X_{1} \leq x, \ldots, X_{n} \leq x\right\} \\
& =\operatorname{Pr}\left\{X_{1} \leq x\right\} \times \ldots \times \operatorname{Pr}\left\{X_{n} \leq x\right\} \\
& =F(x)^{n} .
\end{aligned}
$$

Podemos observar que para $x<x_{+}$, em que $x_{+}$é o menor valor tal que $F(x)=1, F(x)^{n} \rightarrow 0$ quando $n \rightarrow \infty$. Assim a distribuição de $M_{n}$ é degenerada em $x_{+}$. Para contornar este problema, define-se uma normalização linear da variável $M_{n}$ :

$$
M_{n}^{*}=\frac{M_{n}-b_{n}}{a_{n}}
$$

em que $a_{n}$ e $b_{n}$ são sequências de constantes apropriadas para estabilizar os parâmetros de localização e escala de $M_{n}^{*}$ conforme $n$ aumenta e evitar que se tenha uma distribuição degenerada. Se existem sequências de constantes $a_{n}>0$ e $b_{n}$ tais que $\operatorname{Pr}\left\{M_{n}^{*} \leq x\right\} \rightarrow G(x)$, quando $n \rightarrow \infty$, em que $G(x)$ é uma função de distribuição não degenerada $G$ então diz-se que $F$ está no domínio de atração de $G$ e escreve-se que $F \in \mathcal{D}_{\mathcal{M}}(G)$. As distribuições limite possíveis para $M_{n}^{*}$ são dadas no Teorema 1 (Gnedenko, 1943).

Teorema 1. Se existem sequências de constantes $a_{n}>0$ e $b_{n}$ tais que

$$
\operatorname{Pr}\left\{M_{n}^{*} \leq x\right\} \rightarrow G(x), \quad \text { quando } n \rightarrow \infty,
$$

em que $G(x)$ é uma função de distribuição não degenerada $G$, então $G$ pertence à família de distribuições GEV (valor extremo generalizada)

$$
G(x)=\exp \left(-\left[1+\xi\left(\frac{x-\mu}{\sigma}\right)\right]^{-1 / \xi}\right)
$$


definida em $\{x: 1+\xi(x-\mu) / \sigma>0\}$, em que $-\infty<\mu<\infty, \sigma>0 \quad$ e $\quad-\infty<\xi<\infty$.

A distribuição de valor extremo generalizada tem três parâmetros: $\mu$, parâmetro de localização, $\sigma$, parâmetro de escala e $\xi$, parâmetro de forma. A distribuição de valor extremo generalizada também é chamada de distribuição de valor extremo tipo von Mises ou distribuição tipo von Mises-Jenkinson. Para $\xi=0$ (interpretado como o limite de (1.2) quando $\xi \rightarrow 0), \xi>0$ e $\xi<0$ obtemos, respectivamente:

$$
\begin{cases}G(x)=\exp \left(-\exp \left(-\frac{x-\mu}{\sigma}\right)\right), & -\infty<x<\infty \text { se } \xi \rightarrow 0 ; \\ G(x)=\exp \left(-\left[1+\xi\left(\frac{x-\mu}{\sigma}\right)\right]^{-1 / \xi}\right), & \begin{cases}\mu-\sigma / \xi<x<\infty & \text { se } \xi>0 ; \\ -\infty<x<\mu-\sigma / \xi & \text { se } \xi<0 .\end{cases} \end{cases}
$$

Podemos escrever $1+\xi((x-\mu) / \sigma)=(\xi x-(\xi \mu-\sigma)) / \sigma$ e definir a variável aleatória $y=\xi x$ e seu parâmetro de posição $\mu^{*}=(\xi \mu-\sigma)$. Desta forma, as três classes de distribuição acima podem ser escritas como

$$
\left\{\begin{array}{l}
\mathrm{I}: G(x)=\exp \left(-\exp \left(-\frac{x-\mu}{\sigma}\right)\right),-\infty<x<\infty ; \\
\mathrm{II}: G(x)= \begin{cases}0, & x \leq \mu \\
\exp \left(-\left(\frac{x-\mu}{\sigma}\right)^{-1 / \xi}\right), & x>\mu ;\end{cases} \\
\text { III }: G(x)= \begin{cases}\exp \left(-\left(-\frac{x-\mu}{\sigma}\right)^{1 / \xi}\right), & x<\mu \\
1, & x \geq \mu,\end{cases}
\end{array}\right.
$$

em que $\sigma>0$ e $\xi>0$. As três classes de distribuição são chamadas de distribuição valor extremo de tipos I, II e III, também chamadas de famílias Gumbel, Fréchet e Weibull, respectivamente. Observe que as distribuições das famílias Fréchet e Weibull são relacionadas pela simples troca de sinal e que ambas podem ser transformadas numa distribuição da família Gumbel. Isto é, se a variável aleatória $X$ tem distribuição valor extremo tipo II (família Fréchet) então $Z=\ln ((X-\mu) / \sigma)$ tem distribuição Gumbel quando $\xi \rightarrow 0$ e se $X$ tem distribuição tipo III (família Weibull) então $Z=-\ln ((\mu-X) / \sigma)$ tem distribuição Gumbel quando $\xi \rightarrow 0$. As distribuições do tipo I são as mais utilizadas em problemas de valor extremo, também são chamadas de exponencial dupla e alguns autores a chamam de $a$ distribuição do valor extremo. Observe ainda que se $X$ tem distribuição tipo III (família Weibull) então $-X$ tem distribuição Weibull. Embora estas distribuições sejam conhecidas como valor extremo, elas não representam distribuições de todos os tipos de valor extremo (por exemplo, em amostras de tamanho finito) e elas podem ser usadas empiricamente (sem um modelo de valor extremo). Podemos dizer que os três tipos de distribuição do valor extremo são os únicos limites possíveis para a distribuição do valor extremo normalizado $M_{n}^{*}$, independentemente da distribuição da população. Interpretando o limite do Teorema 1 como uma aproximação para valores grandes de $n$, podemos escrever que

$$
\operatorname{Pr}\left\{\frac{M_{n}-b_{n}}{a_{n}} \leq x\right\} \approx G(x)
$$


que é equivalente a

$$
\operatorname{Pr}\left\{M_{n} \leq x\right\} \approx G\left(\frac{x-b_{n}}{a_{n}}\right)=G^{*}(x),
$$

e $G^{*}(x)$ também pertence à família GEV. Isto é, se o Teorema1 permite a aproximação da distribuição de $M_{n}^{*}$ por um membro da família GEV, a distribuição de $M_{n}$ também pode ser aproximada por uma distribuição da família GEV.

Em algumas aplicações o interesse é modelar valores muito pequenos em vez de valores muito grandes. Este não é o caso de problemas na área ambiental, mas modelos de falhas de sistemas podem ser definidos de maneira que o tempo de falha do sistema é o tempo mínimo de falha de seus $n$ componentes. O tempo mínimo de vida do sistema é, então, $\widetilde{M}_{n}=\min \left\{X_{1}, \ldots, X_{n}\right\}$, em que $X_{i}$ é o tempo de vida do componente $i$. Estes sistemas são também chamados de produto do tipo "ligação do mais fraco", isto é, um produto é uma unidade composta de muitas partes, cada uma com um tempo de falha de uma mesma distribuição (limitada inferiormente), e a unidade falha quando uma parte falha. Por exemplo, a vida de um capacitor é pensada como determinada pelo dielétrico mais fraco (com menor tempo de vida) dentre os dielétricos que o compõem. Outro exemplo é o conjunto de dados apresentado em Smith \& Naylor (1987) com as medidas de resistência de fibras de vidro. A distribuição do valor extremo mínimo descreve adequadamente certos tipos de fenômenos como temperatura mínima, chuvas durante períodos de seca, resistência de materiais elétricos, certos tipos de dados de sobrevivência, por exemplo, mortalidade humana dos idosos. A modelagem do tempo mínimo de vida é conhecida como modelagem de confiabilidade. A distribuição do valor extremo máximo raramante é usada para analisar dados de tempo de vida ou falha e tem sido usada para alguns tipos de observações máximas, por exemplo, para altura de inundação, velocidades extrema de ventos e a idade do falecido mais idoso de cada ano em uma comunidade.

Assumindo que $X_{1}, X_{2}, \ldots$ são independentes e identicamente distribuídas, argumentos análogos aos usados em relação ao máximo $M_{n}$ são válidos para a distribuição limite do mínimo $\widetilde{M}_{n}$. Se $Y_{i}=-X_{i}$, para $i=1, \ldots, n$, então, o menor valor de $X_{i}$ corresponde ao maior valor de $Y_{i}$ e se $\widetilde{M}_{n}=\min \left\{X_{1}, \ldots, X_{n}\right\}$ e $M_{n}=\max \left\{Y_{1}, \ldots, Y_{n}\right\}$ temos que $\widetilde{M}_{n}=-M_{n}$ portanto

$$
\begin{aligned}
\operatorname{Pr}\left\{\widetilde{M}_{n} \leq x\right\} & =\operatorname{Pr}\left\{-M_{n} \leq x\right\} \\
& =\operatorname{Pr}\left\{M_{n} \geq-x\right\} \\
& =1-\operatorname{Pr}\left\{M_{n} \leq-x\right\} \\
& \approx 1-\exp \left(-1\left[\xi\left(\frac{-x-\mu}{\sigma}\right)\right]^{-1 / \xi}\right) \\
& =1-\exp \left(-1\left[-\xi\left(\frac{x-\tilde{\mu}}{\sigma}\right)\right]^{-1 / \xi}\right),
\end{aligned}
$$

em que $\{x: 1-\xi(x-\widetilde{\mu}) / \sigma>0\}$ e $\widetilde{\mu}=-\mu$. Esta é a distribuição GEV para o mínimo. Pode-se usar esta característica das distribuições de máximo e mínimo para ajustar um modelo GEV para o máximo de $-x_{1}, \ldots,-x_{n}$ quando $x_{1}, \ldots, x_{n}$ são obtidos de uma distribuição GEV para o mínimo. Os estimadores de máxima verossimilhança são correspondentes, isto é, $\widehat{\widetilde{\mu}}=-\widehat{\mu}$. A versão análoga ao Teorema 1 para a 
distribuição do mínimo é dada a seguir.

Teorema 2. Se existe uma sequência de constantes $a_{n}>0$ e $b_{n}$ tais que

$$
\operatorname{Pr}\left\{\frac{\widetilde{M}_{n}-b_{n}}{a_{n}} \leq x\right\} \rightarrow \widetilde{G}(x) \text { quando } n \rightarrow \infty
$$

para uma função de distribuição não degenerada $\widetilde{G}$, então $\widetilde{G}$ pertence à família de distribuições GEV (valor extremo generalizada) para o mínimo

$$
\widetilde{G}(x)=1-\exp \left(-\left[1-\xi\left(\frac{x-\widetilde{\mu}}{\sigma}\right)\right]^{-1 / \xi}\right),
$$

definida em $\{x: 1-\xi(x-\widetilde{\mu}) / \sigma>0\}$, em que $-\infty<\mu<\infty, \sigma>0$ e $-\infty<\xi<\infty$.

Quando $\xi \rightarrow 0$ então $\widetilde{G}(x) \rightarrow 1-\exp \{-\exp ((x-\widetilde{\mu}) / \sigma)\}$, que é uma distribuição do valor extremo mínimo que, assim como a distribuição do valor extremo máximo, também é chamada de família GEV tipo I ou Gumbel.

Em muitas situações a característica de interesse da unidade experimental pode ser afetada por um ou mais fatores/variáveis explicativas. A omissão de uma variável explicativa importante na análise resultará em um sério desvio na estimação dos parâmetros de interesse. Para incorporar as variáveis explicativas na análise, normalmente utiliza-se um modelo de regressão.

O modelo de regressão valor extremo pode ser definido de forma geral como segue.

Sejam $Y_{1}, \ldots, Y_{n}$ variáveis aleatórias independentes, em que cada $Y_{t}, t=1, \ldots, n$, tem distribuição valor extremo tipo I ou Gumbel do máximo ou do mínimo com parâmetros $\mu_{t}$ e $\sigma_{t}\left(Y_{t} \sim V E\left(\mu_{t}, \sigma_{t}\right)\right)$ com função distribuição e densidade

$$
\begin{gathered}
F_{V E_{\text {max }}}\left(y_{t} ; \mu_{t}, \sigma_{t}\right)=\exp \left(-\exp \left(-\frac{y_{t}-\mu_{t}}{\sigma_{t}}\right)\right), \quad y_{t} \in \mathbb{R}, \\
f_{V E_{\text {max }}}\left(y_{t} ; \mu_{t}, \sigma_{t}\right)=\frac{1}{\sigma_{t}} \exp \left(\left(-\frac{y_{t}-\mu_{t}}{\sigma_{t}}\right)-\exp \left(-\frac{y_{t}-\mu_{t}}{\sigma_{t}}\right)\right), \quad y_{t} \in \mathbb{R},
\end{gathered}
$$

ou

$$
\begin{gathered}
F_{V E_{\text {min }}}\left(y_{t} ; \mu_{t}, \sigma_{t}\right)=1-\exp \left(-\exp \left(\frac{y_{t}-\mu_{t}}{\sigma_{t}}\right)\right), \quad y_{t} \in \mathbb{R}, \\
f_{V E_{\text {min }}}\left(y_{t} ; \mu_{t}, \sigma_{t}\right)=\frac{1}{\sigma_{t}} \exp \left(\left(\frac{y_{t}-\mu_{t}}{\sigma_{t}}\right)-\exp \left(\frac{y_{t}-\mu_{t}}{\sigma_{t}}\right)\right), \quad y_{t} \in \mathbb{R},
\end{gathered}
$$

respectivamente, em que $\mu_{t} \in \mathbb{R}$ e $\sigma_{t}>0$ são os parâmetros de localização e dispersão, respectivamente.

O modelo de regressão valor extremo é definido por (1.5) ou (1.6) e por dois componentes sistemáticos dados por

$$
g\left(\mu_{t}\right)=\eta_{t}=\eta\left(x_{t}, \beta\right)
$$


$\mathrm{e}$

$$
h\left(\sigma_{t}\right)=\delta_{t}=\delta\left(z_{t}, \gamma\right),
$$

em que $\beta=\left(\beta_{1}, \ldots, \beta_{k}\right)^{\top}$ e $\gamma=\left(\gamma_{1}, \ldots, \gamma_{m}\right)^{\top}$ são vetores de parâmetros de regressão desconhecidos $\left(\beta \in \mathbb{R}^{k}\right.$ e $\left.\gamma \in \mathbb{R}^{m}\right)(k<n$ e $m<n)$ referentes a localização e escala, respectivamente. Além disso, $x_{t} \mathrm{e}$ $z_{t}$ são observações em $c_{1}$ e $c_{2}$ covariadas $\left(c_{1}\right.$ e $c_{2} \in \mathbb{R}$ ), respectivamente. Aqui, $\eta(\cdot, \cdot)$ e $\delta(\cdot, \cdot)$ são funções contínuas, duas vezes diferenciáveis no segundo argumento (possivelmente não linear). Finalmente, $g(\cdot)$ e $h(\cdot)$ são funções de ligação conhecidas monótonas e duas vezes diferenciáveis com domínios $\mathbb{R}$ e $\mathbb{R}^{+}$, respectivamente.

Barreto-Souza \& Vasconcellos (2011), Gokarna \& Tsokos (2009) e Branko \& Tsokos (2009) estudaram este modelo com a distribuição (1.5). Barreto-Souza \& Vasconcellos (2011) chamam-na de valor extremo, Gumbel e log-Weibull, Gokarna \& Tsokos (2009) chamam-na de Gumbel e Branko \& Tsokos (2009) de Gumbel e exponencial dupla. Paula \& Rojas (1997), Ferreira da Silva et al. (2008) e Chan et al. (2008) estudaram este modelo com a distribuição (1.6). Paula \& Rojas (1997) e Ferreira da Silva et al. (2008) Ferreira da Silva et al. (2008) chamam esta distribuição de valor extremo e Chan et al. (2008) de valor extremo Tipo I e Gumbel . Casos particulares deste modelo têm nomes particulares ou o nome do modelo geral.

Muita teoria tem sido desenvolvida para esta classe de modelos. Chan et al. (2008) desenvolveram estimação pontual e intervalar para um modelo de regressão valor extremo mínimo linear simples com censura do tipo II (neste caso a função de ligação $h$ é a identidade e a função de regressão $\eta$ é linear). Barreto-Souza \& Vasconcellos (2011) propuseram um modelo de regressão valor extremo máximo geral em que os parâmetros de localização e dispersão são funções possivelmente não-lineares dos parâmetros de regressão. Este é baseado no modelo generalizado não-linear (GNLMs) com covariadas no parâmetro de dispersão proposto por Cordeiro \& Udo (2008), que é uma generalização de modelos lineares generalizados (GLMs) introduzidos por Nelder \& Wedderburn (1972) e GNLMs introduzidos por Cordeiro \& Paula (1989).

A inferência em modelo de regressão valor extremo para grandes amostras está baseada em teoria assintótica. Contudo, a inferência pode ser incorreta se a amostra é pequena. Por exemplo, o teste da razão de verossimilhanças assintótico pode ser bastante distorcido se a amostra não for suficientemente grande. Com o objetivo de melhorar a aproximação da distribuição da estatística do teste pela distribuição de referência, uma distribuição $\chi^{2}$, a estratégia usual é substituir a estatística da razão de verossimilhanças por sua versão corrigida de Bartlett (Lawley, 1956). Se a estatística escore é usada, uma estratégia similar pode ser utilizada, isto é, a estatística de escore pode ser substituída por sua versão corrigida de tipo-Bartlett (Cordeiro \& Ferrari, 1991). As correções de Bartlett e as de tipo-Bartlett dependem de alguns cumulantes de derivadas do logaritmo da verossimilhança e sua obtenção, para a maioria dos modelos, é trabalhosa ou mesmo inviável. Um caso mais simples é quando o parâmetro de dispersão é constante e globalmente ortogonal aos parâmetros da regressão como em modelos lineares generalizados; ver Cordeiro (1987) e Cribari-Neto \& Ferrari (1995). Se o parâmetro de dispersão é modelado, mas os parâmetros de regressão são globalmente ortogonais aos parâmetros restantes, a dedução das correções de Bartlett e tipo-Bartlett é factível. No modelo de regressão valor extremo, no entanto, os parâmetros de regressão não são globalmente ortogonais aos parâmetros de dispersão, isto é, a matriz de informação de Fisher não é diagonal em blocos. 
Por esta razão busca-se, neste trabalho, um ajuste para a estatística da razão de verossimilhanças que seja mais facilmente calculado no modelo de regressão valor extremo.

Considerando ainda a situação de amostras pequenas, obtemos ajustes para a estatística da razão de verossimilhanças sinalizada $(R)$. Esta é utilizada quando o interesse é testar um parâmetro unidimensional, possivelmente na presença de parâmetros de perturbação. Com este teste é possível testar hipóteses unilaterais. A distribuição assintótica de $R$ sob a hipótese nula é normal padrão com erro de ordem $O\left(n^{-1 / 2}\right)$. Barndorff-Nielsen (1986) propôs um ajuste para a estatística da razão de verossimlihanças sinalizada $\left(R^{*}\right)$ cuja ordem de aproximação pela normal padrão é $O\left(n^{-3 / 2}\right)$. No entanto, tal aproximação depende de uma estatística ancilar que nem sempre é fácil de ser definida em função da complexidade do modelo. Para contornar esta dificuldade, algumas aproximações para $R^{*}$ foram propostas. Algumas propostas de aproximação para o ajuste da estatística $R^{*}$ foram feitas por DiCiccio \& Martin (1993), Skovgaard (1996) e Severini (1999), cujas ordens de aproximação são $O\left(n^{-1}\right)$ e oor Fraser et al. (1999), cuja ordem de aproximação é $O\left(n^{-3 / 2}\right)$. Os ajustes obtidos neste trabalho não contemplam o caso de dados censurados.

No final do século 19 uma crescente coleção de dados, tabelas e publicações dos governos, intituições privadas e agências demográficas, de ciências sociais, medicina, biologia, economia e seguros revelaram que a distribuição normal nem sempre é adequada para descrever fenômenos em situações do mundo real. Desde que a coleta e modelagem de dados desempenha um papel fundamental em pesquisa científica, as distribuições de probabilidade são essenciais em análise estatística. Apesar da importância e ampla utilização da distribuição normal clássica, instados pela necessidade de obter melhor ajuste da distribuição aos dados, os pesquisadores se debruçaram sobre a tarefa de propor novas distribuições para a variedade de tipos de dados que surgiam. Como consequência, recentemente um visível progresso foi feito em relação a novas distribuições e suas aplicações em áreas como engenharia, ecologia, economia e ciências biomédicas. Uma ampla gama de distribuições paramétricas é essencial dentre as ferramentas estatísticas pois permitem modelar características como assimetria, caudas pesadas e, em alguns casos, multimodalidade.

Há na literatura vários trabalhos que propõem generalizações de distribuições clássicas amplamente utilizadas. Tais generalizações pretendem introduzir mais flexibilidade (assimetria, curtose, multimodalidade e diversidade de formas) às distribuições visando obter melhores ajustes aos conjuntos de dados menos típicos, isto é, com as características mencionadas mais acentuadas. Por exemplo, há vários trabalhos no sentido de obter distribuições com mais assimetria. Uma história recente das distribuições contínuas assimétricas em geral é apresentada em Kotz \& Vicari (2005) e Arellano-Valle et al. (2006).

Várias destas generalizações têm como caso particular a distribuição Gumbel. A distribuição Gumbel é assimétrica e curtótica mas suas medidas de assimetria e curtose são constantes. Por outro lado, as generalizações que a incluem têm assimetria e curtose flexíveis o que possibilita um melhor ajuste a conjuntos de dados com caudas direitas mais pesadas.

Os objetivos desta tese são:

i. obter um ajuste para o teste da razão de verossimilhanças baseado no trabalho de Skovgaard (2001) para o modelo de regressão valor extremo com dispersão variável em que se permite não linearidade na especificação tanto da média quanto da dispersão;

ii. obter ajustes para o teste da razão de verossimilhanças sinalizada propostos por Barndorff-Nielsen 
(1983), DiCiccio \& Martin (1993), Skovgaard (1996), Severini (1999) e Fraser et al. (1999) para o modelo de regressão valor extremo;

iii. comparar a performance de diferentes testes, para pequenas amostras, através de simulação;

iv. apresentar aplicações em conjuntos de dados reais;

v. relacionar algumas propriedades das principais distribuições que têm como caso particular ou limite a distribuição valor extremo máximo ou Gumbel;

vi. confrontar a qualidade de ajuste das distribuições estudadas a conjuntos de dados de velocidade de vento com presença de valores discrepantes para procurar identificar quais distribuições são úteis nesta situação.

A tese se desenvolve como segue. No Capítulo 2 apresentamos o modelo de regressão valor extremo máximo, a função escore e a matriz de informação, obtemos um ajuste para o teste da razão de verossimilhanças baseado no trabalho de Skovgaard (2001) para o modelo de regressão valor extremo máximo com dispersão variável em que se permite não linearidade na especificação tanto da média quanto da dispersão, apresentamos alguns resultados de simulação, comparando a performance, para pequenas amostras, do teste da razão de verossimilhança original e do teste da razão de verossimilhanças ajustado e apresentamos aplicações a conjunto reais de dados. Algumas conclusões são apresentadas. Os resultados são ainda estendidos para o modelo de regressão valor extremo mínimo.

No Capítulo 3 obtemos ajustes para o teste da razão de verossimilhanças sinalizada propostos por Barndorff-Nielsen (1983), DiCiccio \& Martin (1993), Skovgaard (1996), Severini (1999) e Fraser et al. (1999) para o modelo de regressão valor extremo mencionado acima, apresentamos alguns resultados de simulação que visam avaliar a eficácia dos ajustes obtidos e da estatística usual, para compará-los segundo a probabilidade de erro de tipo I. Aplicações a conjuntos reais de dados e conclusões são apresentadas.

No Capítulo 4 apresentamos uma revisão das distribuições que têm como caso particular ou limite a distribuição valor extremo ou Gumbel. Listamos algumas propriedades que podem diferenciá-las e servir como orientação no momento de eleger um modelo para um conjunto de dados. Comparamos a qualidade de ajuste das distribuições estudadas a dados que exibem valores discrepantes. Apresentamos a conclusão de nossas observações.

Utilizamos as linguagens matriciais de programação Ox (Doornik, 2009), R (R Development Core Team, 2013) e Mathematica (Wolfram Research, 2012). 


\section{Capítulo 2}

\section{Ajuste de Skovgaard para o teste da razão de verossimilhanças em modelos de regressão valor extremo}

\subsection{Modelo de regressão valor extremo máximo}

Sejam $y_{1}, \ldots, y_{n}$ variáveis aleatórias independentes, em que cada $y_{t}, t=1, \ldots, n$, tem distribuição valor extremo máximo com parâmetros $\mu_{t}$ e $\sigma_{t}\left(y_{t} \sim V E\left(\mu_{t}, \sigma_{t}\right)\right)$ com função densidade

$$
f_{V E_{\text {max }}}\left(y_{t} ; \mu_{t}, \sigma_{t}\right)=\frac{1}{\sigma_{t}} \exp \left(\left(-\frac{y_{t}-\mu_{t}}{\sigma_{t}}\right)-\exp \left(-\frac{y_{t}-\mu_{t}}{\sigma_{t}}\right)\right), \quad y_{t} \in \mathbb{R}
$$

em que $\mu_{t} \in \mathbb{R}$ e $\sigma_{t}>0$ são os parâmetros de localização e dispersão, respectivamente. A média, mediana e a variância de $y_{t}$ são

$$
\begin{gathered}
\mathrm{E}\left(y_{t}\right)=\mu_{t}+\mathcal{E} \sigma_{t}, \\
m\left(y_{t}\right)=\mu_{t}-\sigma_{t} \ln \ln 2
\end{gathered}
$$

e

$$
\operatorname{var}\left(y_{t}\right)=\frac{\pi^{2}}{6} \sigma_{t}^{2}
$$

respectivamente, onde $\mathcal{E}$ é a constante de Euler; $\mathcal{E} \approx 0,5772$ (ver Apêndice F.1).

O quantil $y_{t, p}$, tal que $F\left(y_{t, p}\right)=p$, obtido invertendo-se a função de distribuição acumulada dada em (1.4), é dado por

$$
y_{t, p}=\mu_{t}-\sigma_{t} \ln (-\ln (p))
$$

A assimetria e a curtose da valor extremo máximo ou Gumbel não dependem de parâmetros e são iguais a $(12 \sqrt{(6)} \zeta(3)) / \pi^{3} \approx 1,139547$ e 5,4, respectivamente, em que $\zeta(s)=\sum_{k=1}^{\infty} k^{-s}$, Re $s>1$ é a função zeta de Riemann e $\zeta(3) \approx 1,2$ (ver Apêndice F.1).

O modelo de regressão valor extremo máximo com covariadas para a localização e a dispersão é definido por (2.1) e por dois componentes sistemáticos dados por

$$
g\left(\mu_{t}\right)=\eta_{t}=\eta\left(x_{t}, \beta\right)
$$

e

$$
h\left(\sigma_{t}\right)=\delta_{t}=\delta\left(z_{t}, \gamma\right)
$$


onde $\beta=\left(\beta_{1}, \ldots, \beta_{k}\right)^{\top}$ e $\gamma=\left(\gamma_{1}, \ldots, \gamma_{m}\right)^{\top}$ são vetores de parâmetros de regressão desconhecidos $(\beta \in$ $\mathbb{R}^{k}$ e $\left.\gamma \in \mathbb{R}^{m}\right)$ e $x_{t}$ e $z_{t}$ são observações em $c_{1}$ e $c_{2}$ covariadas $\left(c_{1} \leq k<n\right.$ e $\left.c_{2} \leq m<n\right)$. Aqui, $\eta(\cdot, \cdot)$ e $\delta(\cdot, \cdot)$ são funções contínuas, duas vezes diferenciáveis no segundo argumento (possivelmente não lineares). Finalmente, $g(\cdot)$ e $h(\cdot)$ são funções de ligação conhecidas monótonas e duas vezes diferenciáveis com domínios $\mathbb{R}$ e $\mathbb{R}^{+}$, respectivamente. Seja $X$ a matriz de derivadas de $\eta=\left(\eta_{1}, \ldots, \eta_{n}\right)^{\top}$ com respeito a $\beta^{\top}$. Analogamente, seja $Z$ a matriz de derivadas de $\delta=\left(\delta_{1}, \ldots, \delta_{n}\right)^{\top}$ com respeito a $\gamma^{\top}$. Assume-se que $\operatorname{posto}(X)=k$ e posto $(Z)=m$ para todo $\beta$ e $\gamma$. Uma possível escolha para a função de ligação para a dispersão é $h(\sigma)=\ln (\sigma)$.

Seja $\theta=\left(\beta^{\top}, \gamma^{\top}\right)^{\top}$ o vetor de parâmetros desconhecidos do modelo de regressão (2.1)-(2.3). No que segue, $\nu=\left(\nu_{1}, \ldots, \nu_{r}\right)^{\top}$ representa o vetor de parâmetros de interesse e $\psi=\left(\psi_{1}, \ldots, \psi_{s}\right)^{\top}$ é o vetor de parâmetros de perturbação (note que $r+s=k+m$ ). Consideraremos na Seção 2.2 um teste baseado na função de verossimilhança da hipótese nula $\mathcal{H}_{0}: \nu=\nu_{0}$ onde $\nu_{0}$ é um vetor fixo de dimensão $r$. Tal teste pode ser invertido para obter intervalo de confiança para $\nu$.

Seja $\ell(\theta)$ o logaritmo da função de verossimilhança do modelo definido por (2.1)-(2.3) dado o vetor de observações $y=\left(y_{1}, \ldots, y_{n}\right)^{\top}$. Podemos escrever o logaritmo da função de verossimilhança como

$$
\ell(\theta)=\sum_{t=1}^{n} \ell_{t}\left(\mu_{t}, \sigma_{t}\right)
$$

e temos, por (2.1), que

$$
\begin{aligned}
\ell_{t}\left(\mu_{t}, \sigma_{t}\right) & =\ln f\left(y_{t} ; \mu_{t}, \sigma_{t}\right) \\
& =-\ln \sigma_{t}-\frac{y_{t}-\mu_{t}}{\sigma_{t}}-\exp \left(-\frac{y_{t}-\mu_{t}}{\sigma_{t}}\right)
\end{aligned}
$$

com $\mu_{t}$ e $\sigma_{t}$ definidos de tal forma que (2.2) e (2.3) sejam válidos. Portanto, $\ell(\theta)$ pode ser escrito como

$$
\ell(\theta)=\iota^{\top}\left[-\Phi^{*}-\mathcal{Z}-\mathcal{Z}^{\dagger}\right] \iota
$$

onde $\iota$ é o vetor coluna de uns de dimensão $n, \Phi^{*}=\operatorname{diag}\left\{\ln \sigma_{1}, \ldots, \ln \sigma_{n}\right\}, \mathcal{Z}=\operatorname{diag}\left\{z_{1}, \ldots, z_{n}\right\}$, $\mathcal{Z}^{\dagger}=\operatorname{diag}\left\{\exp \left(-z_{1}\right), \ldots, \exp \left(-z_{n}\right)\right\}$ e $z_{t}=\left(y_{t}-\mu_{t}\right) / \sigma_{t}$.

A função escore, obtida diferenciando-se o logaritmo da função de verossimilhança com respeito aos parâmetros desconhecidos, é dada por

$$
U=\left(U_{\beta}(\beta, \gamma)^{\top}, U_{\gamma}(\beta, \gamma)^{\top}\right)^{\top}
$$

em que

$$
U_{\beta}(\beta, \gamma)=X^{\top} \Phi^{-1} T\left(\mathcal{I}-\mathcal{Z}^{\dagger}\right) \iota
$$

$\mathrm{e}$

$$
U_{\gamma}(\beta, \gamma)=Z^{\top} \Phi^{-1} H\left(-\mathcal{I}+\mathcal{Z}-\mathcal{Z} \mathcal{Z}^{\dagger}\right) \iota
$$

onde $\mathcal{I}$ é a matriz identidade $n \times n, \Phi=\operatorname{diag}\left\{\sigma_{1} \ldots, \sigma_{n}\right\}, T=\operatorname{diag}\left\{1 / g^{\prime}\left(\mu_{1}\right) \ldots, 1 / g^{\prime}\left(\mu_{n}\right)\right\}, H=$ 
$\operatorname{diag}\left\{1 / h^{\prime}\left(\sigma_{1}\right) \ldots, 1 / h^{\prime}\left(\sigma_{n}\right)\right\}$,

A matriz de informação observada, negativo da matriz hessiana (segunda derivada) do logaritmo da função de verossimilhança em relação aos parâmetros desconhecidos, é dada por

$$
J=\left[\begin{array}{cc}
J_{\beta \beta} & J_{\beta \gamma} \\
J_{\gamma \beta} & J_{\gamma \gamma}
\end{array}\right]
$$

com

$$
\begin{gathered}
J_{\beta \beta}=X^{\top} \Phi^{-1} T\left(\mathcal{Z}^{\dagger} \Phi^{-1}+\left(\mathcal{I}-\mathcal{Z}^{\dagger}\right) S T\right) T X-\left[\iota^{\top}\left(\mathcal{I}-\mathcal{Z}^{\dagger}\right) T \Phi^{-1}\right][\dot{X}], \\
J_{\beta \gamma}=J_{\gamma \beta}^{\top}=X^{\top} \Phi^{-1} T\left(\mathcal{I}-\mathcal{Z}^{\dagger}+\mathcal{Z} \mathcal{Z}^{\dagger}\right) H \Phi^{-1} Z
\end{gathered}
$$

e

$$
\begin{aligned}
J_{\gamma \gamma}= & Z^{\top} \Phi^{-1} H\left(\left(-\mathcal{I}+2 \mathcal{Z}-2 \mathcal{Z} \mathcal{Z}^{\dagger}+\mathcal{Z}^{2} \mathcal{Z}^{\dagger}\right) \Phi^{-1}+\left(-\mathcal{I}+\mathcal{Z}-\mathcal{Z Z}^{\dagger}\right) Q H\right) H Z+ \\
& {\left[\iota^{\top}\left(\mathcal{I}-\mathcal{Z}+\mathcal{Z} \mathcal{Z}^{\dagger}\right) H \Phi^{-1}\right][\dot{Z}] . }
\end{aligned}
$$

onde $S=\operatorname{diag}\left\{g^{\prime \prime}\left(\mu_{1}\right), \ldots, g^{\prime \prime}\left(\mu_{n}\right)\right\}, Q=\operatorname{diag}\left\{h^{\prime \prime}\left(\sigma_{1}\right), \ldots, h^{\prime \prime}\left(\sigma_{n}\right)\right\}, \dot{X}=\partial^{2} \eta / \partial \beta \partial \beta^{\top}$ e $\dot{Z}=$ $\partial^{2} \delta / \partial \gamma \partial \gamma^{\top}$ representando $n \times k \times k$ e $n \times m \times m$ arrays respectivamente. Aqui, [.] [.] representa o produto 'colchete' de uma matriz por um array, como definido por Wei (1998, p. 188). Mais especificamente, se $A$ é uma matriz $m \times n$ e $B$ é um array $n \times p \times q$, então $C=[A][B]$ é chamado o produto 'colchete' de $A$ por $B$, que é um array $m \times p \times q$ com elementos $C_{t i j}=\sum_{k=1}^{n} A_{t k} B_{k i j}$ (ver Apêndice A).

A matriz de informação de Fisher, que é a esperança da matriz de informação observada, é dada por

$$
I=\left[\begin{array}{cc}
I_{\beta \beta} & I_{\beta \gamma} \\
I_{\gamma \beta} & I_{\gamma \gamma}
\end{array}\right]
$$

com

$$
\begin{gathered}
I_{\beta \beta}=X^{\top} \Phi^{-1} T^{2} \Phi^{-1} X \\
I_{\beta \gamma}=I_{\gamma \beta}^{\top}=(\mathcal{E}-1) X^{\top} \Phi^{-1} T H \Phi^{-1} Z
\end{gathered}
$$

$\mathrm{e}$

$$
I_{\gamma \gamma}=\left(1+\Gamma^{(2)}(2)\right) Z^{\top} \Phi^{-1} H^{2} \Phi^{-1} Z
$$

em que $\Gamma(\cdot)^{(2)}$ é a segunda derivada da função Gamma, $\Gamma(x)=\int_{0}^{\infty} e^{-t} t^{x-1} d t$ e Re $x>0$ (ver Apêndice A).

Sob condições de regularidade, $\ell(\theta)$ é diferenciável em $\theta$, e o estimador de máxima verossimilhança $\widehat{\theta}$ existe e é tal que

$$
U(\widehat{\theta})=0
$$


e

$$
\ell(\widehat{\theta}) \geq \ell(\theta), \quad \forall \theta \in \Theta
$$

Lehmann \& Casella (1998, cap. 6) mostram que, sob condições de regularidade, a raiz da equação (2.18), se única, tem as seguintes propriedades:

i - é consistente;

ii - é assintoticamente eficiente, isto é, dentre os estimadores consistentes de $\theta$, é o que possui variância assintótica mínima;

iii - é invariante sob transformações biunívocas, isto é, se $\widehat{\theta}$ é o estimador de máxima verossimilhança de $\theta$ e $g$ é uma função bijetora de $\theta$, então $g(\widehat{\theta})$ é o estimador de máxima verossimilhança de $g(\theta)$;

iv - tem distribuição assintótica normal $(k+m)$-variada, de tal forma que $\widehat{\theta} \stackrel{a}{\sim} \mathcal{N}_{k+m}\left(\theta, I(\theta)^{-1}\right)$;

v - geralmente, é viesado mas assintoticamente não-viesado.

Como, neste caso, não é possível expressar os estimadores de máxima verossimilhança em uma forma fechada, é necessário utilizar técnicas iterativas de otimização e os estimadores de máxima verossimilhança de $\beta$ e $\gamma$ são obtidos numericamente maximizando-se o logaritmo da função de verossimilhança usando um algorithmo de otimização não linear, como por exemplo um algoritmo de Newton (Newton-Raphson, Fisher's scoring, BHHH, etc.) ou um algoritmo quasi-Newton (BFGS, etc.).

Para obter os estimadores de máxima verossimilhança dos parâmetros pode-se usar os softwares disponíveis. Sugerimos o site http: / / www.ral.ucar.edu/\$ thicksim\$ericg/softextreme.php, onde há uma relação de funções para análise de valor extremo em diferentes softwares.

No software R (R Development Core Team, 2013) temos os seguintes pacotes: (ver http://cran.r-project.org/web/packages/available_packages_by_name.html)

- pacote gamlss : função gamlss - ajuste pelo estimador de máxima verossimilhança (penalizada) para modelo de regressão aditivo generalizado. É possível modelar os parâmetros de localização, escala e forma com variável resposta com distribuição Gumbel do valor extremo mínimo, com modelo linear/não linear paramétricos e/ou funções aditivas não paramétricas e/ou termos de efeito aleatório. É possível fixar parâmetros. Exemplos podem ser encontrados em: http: //www . jstat soft.org/ v23/i07/supp/7.

- pacote ismev : funções gum.fit e gev.fit - ajuste pelo estimador de máxima verossimilhança para a distribuição Gumbel do valor extremo máximo e valor extremo generalizado, respectivamente, incluindo modelo linear generalizado para cada parâmetro. Funções e conjuntos de dados utilizados nos exemplos apresentados em Coles (2001).

- pacote evd : função fgev - ajuste pelo estimador de máxima verossimilhança para a distribuição valor extremo generalizada do máximo $\left(G(x)=\exp \left[-(1+s(z-a) / b)^{-1 / s}\right]\right)$, permitindo modelo linear para o parâmetro de localização e parâmetros fixos. Como caso particular, se o parâmetro s=0 (shape), temos um ajuste pela distribuição Gumbel. 
- pacote SpatialExtremes : função fitspatgev - ajuste pelo estimador de máxima verossimilhança para modelo de regressão valor extremo linear nos parâmetros de localização, escala e forma. Não é possível fixar parâmetros.

\subsection{Ajuste de Skovgaard - valor extremo máximo}

A estatística da razão de verossimilhanças para testar $\mathcal{H}_{0}: \nu=\nu_{0}$ pode ser escrita como

$$
w=2\left\{\ell(\widehat{\nu}, \widehat{\psi})-\ell\left(\nu_{0}, \widetilde{\psi}\right)\right\}
$$

Aqui, $\left(\widehat{\nu}^{\top}, \widehat{\psi}^{\top}\right)$ é o estimador de máxima verossimilhança de $\left(\nu^{\top}, \psi^{\top}\right)$ e $\left(\nu_{0}^{\top}, \widetilde{\psi}^{\top}\right)$ é o estimador de máxima verossimilhança restrito de $\left(\nu^{\top}, \psi^{\top}\right)$ sob $\mathcal{H}_{0}$, com $\left(\nu^{\top}, \psi^{\top}\right)$ tal como definido na Seção 2.1. Em grandes amostras, $w$ é aproximadamente distribuído como $\chi_{r}^{2}$ sob $\mathcal{H}_{0}$ com erro de ordem $n^{-1}$. Contudo, sabe-se que o teste da razão de verossimilhanças pode ter tamanho substancialmente distorcido se a amostra não for suficientemente grande para garantir uma boa aproximação da distribuição nula de $w$ pela distribuição $\chi^{2}$ de referência. O objetivo deste capítulo é obter uma estatística de razão de verossimilhanças ajustada cuja distribuição sob $\mathcal{H}_{0}$ seja bem aproximada por uma distribuição $\chi^{2}$ mesmo em amostras pequenas ou de tamanho moderado.

Skovgaard (2001) deduziu uma estatística de razão de verossimilhanças ajustada dada por

$$
w^{*}=w-2 \ln \xi
$$

onde

$$
\xi=\frac{\left\{|\widetilde{I}||\widehat{I}|\left|\widetilde{J}_{\psi \psi}\right|\right\}^{1 / 2}}{|\bar{\Upsilon}|\left|\left[\widetilde{I} \bar{\Upsilon}^{-1} \widehat{J} \widehat{I}^{-1} \bar{\Upsilon}\right]_{\psi \psi}\right|^{1 / 2}} \frac{\left\{\widetilde{U}^{\top} \bar{\Upsilon}^{-1} \widehat{I} \widehat{J}^{-1} \bar{\Upsilon} \widetilde{I}^{-1} \widetilde{U}\right\}^{r / 2}}{w^{r / 2-1} \widetilde{U}^{\top} \bar{\Upsilon}^{-1} \bar{q}}
$$

e $\bar{q}$ e $\bar{\Upsilon}$ são obtidos a partir de

$$
q\left(\omega_{1}, \omega\right)=\mathrm{E}_{\omega_{1}}\left[U\left(\omega_{1}\right)\left(\ell\left(\omega_{1}\right)-\ell(\omega)\right)\right]
$$

$\mathrm{e}$

$$
\Upsilon\left(\omega_{1}, \omega\right)=\mathrm{E}_{\omega_{1}}\left[U\left(\omega_{1}\right) U^{\top}(\omega)\right]
$$

substituindo $\omega_{1}$ pelo estimador de máxima verossimilhança de $\omega(\widehat{\omega})$ e $\omega$ pelo estimador de máxima verossimilhança de $\omega$ com restrição sob $\mathcal{H}_{0}(\widetilde{\omega})$, respectivamente, depois que os valores esperados são calculados, isto é, $\bar{q}=q(\widehat{\theta}, \widetilde{\theta})$ e $\bar{\Upsilon}=\Upsilon(\widehat{\theta}, \widetilde{\theta})$, em que $\theta$ é o vetor de parâmetros desconhecidos do modelo. O subescrito indica que a esperança matemática é calculada considerando $\omega_{1}$ como o verdadeiro parâmetro. Uma matriz $A_{\psi \psi}$ é uma matriz formada a partir da matriz $A,(r+s) \times(r+s)$, excluindo as linhas e colunas correspondentes ao parâmetro de interesse. Assim $J_{\psi \psi}$ é a matriz de informação observada $s \times s$ correspondente a $\psi$. Além disso, $\quad \hat{r}$ e $\sim$ indicam avaliação no estimador de máxima verossimilhança de $\omega(\widehat{\omega})$ e com restrição sob $\mathcal{H}_{0}(\widetilde{\omega})$ respectivamente. Por exemplo, $\widehat{I}=I(\widehat{\omega}), \widetilde{I}=I(\widetilde{\omega})$ e $\widehat{J}=J(\widehat{\omega})$.

Note que $\bar{q}$ é um vetor de dimensão $(r+s)$ e $\bar{\Upsilon}$ é uma matriz de dimensão $(r+s) \times(r+s)$. Sob $\mathcal{H}_{0}, w^{*}$ 
é aproximadamente distribuída como $\chi_{r}^{2}$ com alto grau de acurácia (Skovgaard, 2001, p. 7). A estatística

$$
w^{* *}=w\left(1-\frac{1}{w} \ln \xi\right)^{2}
$$

é assintoticamente equivalente a $w^{*}$. Como assinalado por Skovgaard, a versão $w^{*}$ é a que surge a partir do desenvolvimento teórico. Através de simulações, Skovgaard mostrou que $w^{*}$ tem uma performance um pouco melhor que $w^{* *}$ em alguns casos. Ferrari \& Pinheiro (2011) concluíram que, no modelo de regressão beta, as taxas de rejeição da hipótese nula dos testes que usam as estatísticas ajustadas são próximas dos respectivos níveis nominais, com uma pequena vantagem para o teste baseado em $w^{*}$. Contudo, diferentemente de $w^{*}, w^{* *}$ é sempre não negativa. A estatística $R^{*}$ (Barndorff-Nielsen, 1986, 1991) é uma versão modificada da estatística da razão de verossimilhanças que tem distribuição normal sob a hipótese nula com erro de ordem $n^{-3 / 2}$, enquanto o erro desta aproximação é $n^{-1 / 2}$ quando a estatística não ajustada é usada. Estas informações podem ser conferidas em Skovgaard (2001, Seção 5.7), onde são apresentadas com mais detalhes. Note-se que, quando $r=1$, a estatística $w^{* *}$ se reduz a $R^{* 2}$. Assim como $w$, as estatísticas ajustadas são invariantes sob reparametrização da forma $(\nu, \psi) \longrightarrow(\nu, \zeta(\nu, \psi))$.

Para obter a estatística da razão de verossimilhanças ajustada $w^{*}$ no modelo de regressão valor extremo máximo (2.1)-(2.3), é necessário obter o vetor $q$ e a matriz $\Upsilon$. A obtenção detalhada dos resultados é dada no Apêndice B. Temos que

$$
\bar{q}=\left[\begin{array}{c}
X_{1}^{\top} \Phi_{1}^{-1} T_{1} C\left(\mathcal{I}-M D^{\dagger}\right) \iota \\
Z_{1}^{\top} \Phi_{1}^{-1} H_{1}\left(C\left(\mathcal{E} \mathcal{I}+N D^{\dagger}\right)-\mathcal{I}\right) \iota
\end{array}\right]
$$

$\mathrm{e}$

$$
\bar{\Upsilon}=\left[\begin{array}{cc}
X_{1}^{\top} \Phi_{1}^{-1} T_{1} C M D^{\dagger} T \Phi^{-1} X & X_{1}^{\top} \Phi_{1}^{-1} T_{1} C\left\{\mathcal{I}+D^{\dagger}(-M-C N+M D)\right\} H \Phi^{-1} Z \\
-Z_{1}^{\top} \Phi_{1}^{-1} H_{1} C N D^{\dagger} T \Phi^{-1} X & Z_{1}^{\top} \Phi_{1}^{-1} H_{1} C\left\{\mathcal{E} \mathcal{I}+D^{\dagger}(N+C P-N D)\right\} H \Phi^{-1} Z
\end{array}\right],
$$

em que $C=\operatorname{diag}\left\{\sigma_{11} / \sigma_{1}, \ldots, \sigma_{1 n} / \sigma_{n}\right\}, D=\operatorname{diag}\left\{\left(\mu_{11}-\mu_{1}\right) / \sigma_{1}, \ldots,\left(\mu_{1 n}-\mu_{n}\right) / \sigma_{n}\right\}, D^{\dagger}=$ $\operatorname{diag}\left\{\exp \left(-\left(\mu_{11}-\mu_{1}\right) / \sigma_{1}\right), \ldots, \exp \left(-\left(\mu_{1 n}-\mu_{n}\right) / \sigma_{n}\right)\right\}, M=\operatorname{diag}\left\{\Gamma\left(1+\sigma_{11} / \sigma_{1}\right), \ldots, \Gamma\left(1+\sigma_{1 n} / \sigma_{n}\right)\right\}$, $N=\operatorname{diag}\left\{\Gamma^{(1)}\left(1+\sigma_{11} / \sigma_{1}\right), \ldots, \Gamma^{(1)}\left(1+\sigma_{1 n} / \sigma_{n}\right)\right\}, P=\operatorname{diag}\left\{\Gamma^{(2)}\left(1+\sigma_{11} / \sigma_{1}\right), \ldots, \Gamma^{(2)}\left(1+\sigma_{1 n} / \sigma_{n}\right)\right\}$, e as outras matrizes foram definidas anteriormente.

$$
\bar{q}=\left[\begin{array}{c}
\widehat{X}^{\top} \widehat{\Phi}^{-1} \widehat{T} \bar{C}\left(\mathcal{I}-\bar{M} \bar{D}^{\dagger}\right) \iota \\
\widehat{Z}^{\top} \widehat{\Phi}^{-1} \widehat{H}\left\{\bar{C}\left(\mathcal{E} \mathcal{I}+\bar{N} \bar{D}^{\dagger}\right)-\mathcal{I}\right\} \iota
\end{array}\right]
$$

$\mathrm{e}$

$$
\bar{\Upsilon}=\left[\begin{array}{cc}
\widehat{X}^{\top} \widehat{\Phi}^{-1} \widehat{T} \bar{M} \bar{M} \bar{D}^{\dagger} \widetilde{T} \widetilde{\Phi}^{-1} \widetilde{X} & \widehat{X}^{\top} \widehat{\Phi}^{-1} \widehat{T} C\left\{\mathcal{I}+\bar{D}^{\dagger}(\bar{M} \bar{D}-\bar{M}-\bar{C} \bar{N})\right\} \widetilde{H} \widetilde{\Phi}^{-1} \widetilde{Z} \\
-\widehat{Z}^{\top} \widehat{\Phi}^{-1} \widehat{H} \bar{C} \bar{N} \bar{D}^{\dagger} \widetilde{T} \widetilde{\Phi}^{-1} \widetilde{X} & \widehat{Z}^{\top} \widehat{\Phi}^{-1} \widehat{H} \bar{C}\left\{\mathcal{E} \mathcal{I}+\bar{D}^{\dagger}(\bar{N}+\bar{C} \bar{P}-\bar{N} \bar{D})\right\} \widetilde{H} \widetilde{\Phi}^{-1} \widetilde{Z}
\end{array}\right]
$$

em que $\bar{C}=\operatorname{diag}\left\{\widehat{\sigma}_{1} / \widetilde{\sigma}_{1}, \ldots, \widehat{\sigma}_{n} / \widetilde{\sigma}_{n}\right\}, \bar{D}=\operatorname{diag}\left\{\left(\widehat{\mu}_{1}-\widetilde{\mu}_{1}\right) / \widetilde{\sigma}_{1}, \ldots,\left(\widehat{\mu}_{n}-\widetilde{\mu}_{n}\right) / \widetilde{\sigma}_{n}\right\}, \bar{D}^{\dagger}=$ $\operatorname{diag}\left\{\exp \left(-\left(\widehat{\mu}_{1}-\widetilde{\mu}_{1}\right) / \widetilde{\sigma}_{1}\right), \ldots, \exp \left(-\left(\widehat{\mu}_{n}-\widetilde{\mu}_{n}\right) / \widetilde{\sigma}_{n}\right)\right\}, \bar{M}=\operatorname{diag}\left\{\Gamma\left(1+\widehat{\sigma}_{1} / \widetilde{\sigma}_{1}\right), \ldots, \Gamma\left(1+\widehat{\sigma}_{n} / \widetilde{\sigma}_{n}\right)\right\}$, 
$\bar{N}=\operatorname{diag}\left\{\Gamma^{(1)}\left(1+\widehat{\sigma}_{1} / \widetilde{\sigma}_{1}\right), \ldots, \Gamma^{(1)}\left(1+\widehat{\sigma}_{n} / \widetilde{\sigma}_{n}\right)\right\}, \bar{P}=\operatorname{diag}\left\{\Gamma^{(2)}\left(1+\widehat{\sigma}_{1} / \widetilde{\sigma}_{1}\right), \ldots, \Gamma^{(2)}\left(1+\widehat{\sigma}_{n} / \widetilde{\sigma}_{n}\right)\right\}, \mathrm{e}$ as outras matrizes foram definidas anteriormente.

Vale ressaltar que a estatística da razão de verossimilhanças ajustada pode ser facilmente calculada com os recursos de software existentes. Estas requerem apenas a estimação dos parâmetros desconhecidos por máxima verossimilhança e álgebra usual de matrizes.

Quando as estimativas de máxima verossimilhança restrita e irrestrita são iguais, temos que $w=0$ e $\bar{q}$ é um vetor nulo, e as fórmulas para as estatísticas ajustadas não fazem sentido. Usando recurso similar ao utilizado por (Severini, 2000, p. 244) faz-se $w^{*}$ igual a $w$ quando $w<0.1$. Em situações práticas, valores de $w$ próximos de zero indicam que a hipótese nula não deve ser rejeitada, isto é, os $p$-valores são claramente grandes. Em tais situações, não há necessidade de ajustes.

Usualmente, testes de hipótese são baseados nas estatísticas de razão de verossimilhanças, Wald e escore. As estatísticas de Wald e escore de Rao são dadas, respectivamente, por

$$
W=\left(\widehat{\nu}-\nu_{0}\right)^{\top}\left(\widehat{I}^{\nu \nu}\right)^{-1}\left(\widehat{\nu}-\nu_{0}\right)
$$

e

$$
S_{R}=\widetilde{U}_{\nu}^{\top} \widetilde{I}^{\nu \nu} \widetilde{U}_{\nu}
$$

Recentemente, Terrell (2002), propôs uma estatística de teste denominada gradiente. Esta estatística é derivada das estatísticas escore e Wald e é dada por

$$
S_{T}=\widetilde{U}_{\nu}^{\top}\left(\widehat{\nu}-\nu_{0}\right)
$$

Note que a estatística gradiente é muito fácil de calcular já que não envolve a matriz de informação, nem observada nem esperada e tampouco envolve inversa de matriz. Lemonte \& Ferrari (2011) compararam, através de simulação, os testes da razão de verossimilhanças, Wald, escore e gradiente no modelo de regressão Birnbaum-Saunders e concluíram que as distribuições das estatísticas gradiente e escore, estão mais próximas da distribuição de referência quando a amostra é pequena ou de tamanho moderado enquanto os testes da razão de verossimilhanças e Wald podem ser bastante liberais neste caso, e ainda, que todos os quatro testes têm comportamento similar em relação ao poder quando são usados os valores críticos estimados. Lemonte \& Ferrari (2012) compararam os poderes locais dos testes baseados nas estatísticas da razão de verossimilhanças, Wald, escore e gradiente e concluiram que não é possível afirmar em geral que um dos testes é mais poderoso, ou ainda, somente em situações particulares podem ser feitas comparações.

\subsection{Modelo de regressão valor extremo mínimo}

Sejam $y_{1}, \ldots, y_{n}$ variáveis aleatórias independentes, em que cada $y_{t}, t=1, \ldots, n$, tem distribuição valor extremo mínimo com parâmetros $\mu_{t}$ e $\sigma_{t}\left(y_{t} \sim V E_{\min }\left(\mu_{t}, \sigma_{t}\right)\right)$ com função densidade

$$
f_{V E_{\min }}\left(y_{t} ; \mu_{t}, \sigma_{t}\right)=\frac{1}{\sigma_{t}} \exp \left(\left(\frac{y_{t}-\mu_{t}}{\sigma_{t}}\right)-\exp \left(\frac{y_{t}-\mu_{t}}{\sigma_{t}}\right)\right), \quad y_{t} \in \mathbb{R}
$$


onde $\mu_{t} \in \mathbb{R}$ and $\sigma_{t}>0$ são os parâmetros de locação e dispersão, respectivamente. A média e a variância de $y_{t}$ são

$$
\mathrm{E}\left(y_{t}\right)=\mu_{t}-\mathcal{E} \sigma_{t}
$$

$\mathrm{e}$

$$
\operatorname{var}\left(y_{t}\right)=\frac{\pi^{2}}{6} \sigma_{t}^{2}
$$

respectivamente, onde $\mathcal{E}$ é a constante de Euler; $\mathcal{E} \approx 0.5772$.

O modelo de regressão valor extremo mínimo com covariadas para a dispersão é definido por (2.28) e por dois componentes sistemáticos dados por (2.2) e (2.3).

Sob as mesmas condições definidas no Capítulo 2.1 obtemos, de forma análoga ao modelo (2.1)-(2.3), que

$$
\begin{gathered}
\ell_{t, \text { min }}\left(\mu_{t}, \sigma_{t}\right)=\ln \left(f\left(y_{t} ; \mu_{t}, \sigma_{t}\right)\right)=-\ln \sigma_{t}+\left(\frac{y_{t}-\mu_{t}}{\sigma_{t}}\right)-\exp \left(\frac{y_{t}-\mu_{t}}{\sigma_{t}}\right) \\
U_{\beta, \text { min }}(\beta, \gamma)=-X^{\top} \Phi^{-1} T\left(\mathcal{I}-\mathcal{Z}^{*}\right) \iota \\
U_{\gamma}(\beta, \gamma)=Z^{\top} \Phi^{-1} H\left(-\mathcal{I}-\mathcal{Z}+\mathcal{Z} \mathcal{Z}^{*}\right) \iota \\
J_{\beta \beta, \text { min }}=X^{\top} \Phi^{-1} T\left(\mathcal{Z}^{*} \Phi^{-1}-\left(\mathcal{I}-\mathcal{Z}^{*}\right) S T\right) T X+\left[\iota^{\top}\left(\mathcal{I}-\mathcal{Z}^{*}\right) T \Phi^{-1}\right][\dot{X}] \\
J_{\beta \gamma \text { min }}=J_{\gamma \beta \min }^{\top}=-X^{\top} \Phi^{-1} T\left(\mathcal{I}-\mathcal{Z}^{*}-\mathcal{Z} \mathcal{Z}^{*}\right) H \Phi^{-1} Z \\
J_{\gamma \gamma, \text { min }}=Z^{\top} \Phi^{-1} H\left(\left(-\mathcal{I}-2 \mathcal{Z}+2 \mathcal{Z} \mathcal{Z}^{*}+\mathcal{Z}^{2} \mathcal{Z}^{*}\right) \Phi^{-1}+\left(-\mathcal{I}-\mathcal{Z}+\mathcal{Z} \mathcal{Z}^{*}\right) Q H\right) H Z+ \\
{\left[\iota^{\top}\left(\mathcal{I}+\mathcal{Z}-\mathcal{Z} \mathcal{Z}^{*}\right) H \Phi^{-1}\right][\dot{Z}]} \\
I_{\beta \beta, \text { min }}=X^{\top} \Phi^{-1} T^{2} \Phi^{-1} X \\
I_{\beta \gamma, \text { min }}=I_{\gamma \beta}^{\top}=-(\mathcal{E}-1) X^{\top} \Phi^{-1} T H \Phi^{-1} Z
\end{gathered}
$$

$\mathrm{e}$

$$
I_{\gamma \gamma, \min }=\left(1+\Gamma^{(2)}(2)\right) Z^{\top} \Phi^{-1} H^{2} \Phi^{-1} Z
$$

em que $\mathcal{Z}^{*}=\operatorname{diag}\left\{\exp \left(\left(y_{1}-\mu_{1}\right) / \sigma_{1}\right), \ldots, \exp \left(\left(y_{n}-\mu_{n}\right) / \sigma_{n}\right)\right\}=\operatorname{diag}\left\{\exp \left(\mathfrak{z}_{1}\right), \ldots, \exp \left(\mathfrak{z}_{n}\right)\right\}$ e $S, Q$, $\dot{X}, \dot{Z}$ e $[\cdot][\cdot]$ são como definidos no Capítulo 2.1 .

Se $y_{t}, t=1, \ldots, n$, tem distribuição valor extremo mínimo com parâmetros $\mu_{t}$ e $\sigma_{t}\left(y_{t} \sim\right.$ $\left.V E_{\min }\left(\mu_{t}, \sigma_{t}\right)\right)$ então $-y_{t}, t=1, \ldots, n$, tem distribuição valor extremo máximo com parâmetros $-\mu_{t}$ e $\sigma_{t}\left(-y_{t} \sim V E_{\max }\left(-\mu_{t}, \sigma_{t}\right)\right)$. 
Temos, por (2.5) e (2.29), que

$$
\begin{aligned}
\ell_{t, \max }\left(-\mu_{t}, \sigma_{t},-y_{t}\right) & =-\ln \sigma_{t}-\frac{-y_{t}-\left(-\mu_{t}\right)}{\sigma_{t}}-\exp \left(-\frac{-y_{t}-\left(-\mu_{t}\right)}{\sigma_{t}}\right) \\
& =-\ln \sigma_{t}+\frac{y_{t}-\mu_{t}}{\sigma_{t}}-\exp \left(\frac{y_{t}-\mu_{t}}{\sigma_{t}}\right) \\
& =\ell_{t, \min }\left(\mu_{t}, \sigma_{t}, y_{t}\right) .
\end{aligned}
$$

Temos, por (2.2), (2.4) e (A.1),

$$
\frac{\partial \ell_{\max }(\beta, \gamma)}{\partial \beta_{i}}\left(\mu_{t}, \sigma_{t}, y_{t}\right)=\sum_{t=1}^{n}\left\{\left(\frac{1}{\sigma_{t}}-\frac{1}{\sigma_{t}} \exp \left(-\frac{y_{t}-\mu_{t}}{\sigma_{t}}\right)\right) \frac{1}{g^{\prime}\left(\mu_{t}\right)} \frac{\partial \eta_{t}}{\partial \beta_{i}}\right\},
$$

(ver Apêndice A). Então

$$
\begin{aligned}
\frac{\partial \ell_{\max }(\beta, \gamma)}{\partial \beta_{i}}\left(-\mu_{t}, \sigma_{t},-y_{t}\right) & =\sum_{t=1}^{n}\left\{\left(\frac{1}{\sigma_{t}}-\frac{1}{\sigma_{t}} \exp \left(-\frac{-y_{t}-\left(-\mu_{t}\right)}{\sigma_{t}}\right)\right) \frac{1}{g^{\prime}\left(-\mu_{t}\right)} \frac{\partial \eta_{t}}{\partial \beta_{i}}\right\} \\
& =\sum_{t=1}^{n}\left\{\left(\frac{1}{\sigma_{t}}-\frac{1}{\sigma_{t}} \exp \left(\frac{y_{t}-\mu_{t}}{\sigma_{t}}\right)\right) \frac{1}{g^{\prime}\left(-\mu_{t}\right)} \frac{\partial \eta_{t}}{\partial \beta_{i}}\right\}
\end{aligned}
$$

Observe que se $g(\mu)=\eta(\beta)$ então $\mu=\left(g^{-1} \circ \eta\right)(\beta)$, então podemos supor, sem perda de generalidade, que $g$ é a função identidade e o preditor é $\eta^{*}=\left(g^{-1} \circ \eta\right)$, neste caso $T=\operatorname{diag}\left\{1 / g^{\prime}\left(\mu_{1}\right) \ldots, 1 / g^{\prime}\left(\mu_{n}\right)\right\}=\mathcal{I}$ e $S=\operatorname{diag}\left\{g^{\prime \prime}\left(\mu_{1}\right), \ldots, g^{\prime \prime}\left(\mu_{n}\right)\right\}=0_{n \times n}$. Se $-\mu=\eta(\beta)$ então $\mu=-\eta(\beta)$,

$$
\begin{aligned}
\frac{\partial \ell_{\max }(\beta, \gamma)}{\partial \beta_{i}}\left(-\mu_{t}, \sigma_{t},-y_{t}\right) & =\sum_{t=1}^{n}\left\{\left(\frac{1}{\sigma_{t}}-\frac{1}{\sigma_{t}} \exp \left(\frac{y_{t}-\mu_{t}}{\sigma_{t}}\right)\right) \frac{\partial \eta_{t}}{\partial \beta_{i}}\right\} \\
& =\sum_{t=1}^{n}\left\{\left(-\frac{1}{\sigma_{t}}+\frac{1}{\sigma_{t}} \exp \left(\frac{y_{t}-\mu_{t}}{\sigma_{t}}\right)\right) \frac{\partial\left(-\eta_{t}\right)}{\partial \beta_{i}}\right\} \\
& =\frac{\partial \ell_{\min }(\beta, \gamma)}{\partial \beta_{i}}\left(\mu_{t}, \sigma_{t}, y_{t}\right) .
\end{aligned}
$$

Então $\hat{\beta}_{\text {max }}(-y)=\hat{\beta}_{\text {min }}(y)$ se definirmos o preditor $\eta^{*}(\beta,-\mu)=-\eta(\beta, \mu)$. Temos ainda que

$$
\begin{aligned}
\mathfrak{z}^{\dagger}(-y)= & \left.\left.\left(\exp \left(-\left(-y_{1}-\left(-\mu_{1}\right)\right)\right) / \sigma_{1}\right), \ldots, \exp \left(-\left(-y_{n}-\left(-\mu_{n}\right)\right)\right) / \sigma_{n}\right)\right) \\
= & \left(\exp \left(\left(y_{1}-\mu_{1}\right) / \sigma_{1}\right) \ldots, \exp \left(-\left(y_{n}-\mu_{n}\right) / \sigma_{n}\right)\right)=\mathfrak{z}^{*}(y), \\
& \mathcal{Z}^{\dagger}(-y)=\operatorname{diag}\left\{\exp \left(\mathfrak{z}_{1}\right), \ldots, \exp \left(\mathfrak{z}_{n}\right)\right\}=\mathcal{Z}^{*}(y)
\end{aligned}
$$

e

$$
X(-y)=\left(\frac{\partial \eta_{i}\left(-\mu_{i}, \sigma_{i}\right)}{\partial \beta_{j}}\right)=-\left(\frac{\partial \eta_{i}\left(\mu_{i}, \sigma_{i}\right)}{\partial \beta_{j}}\right)=-X(y) .
$$

Por (2.8),(2.9), (2.11), (2.12), (2.13), (2.15), (2.16), (2.17) e considerando $g$ a função identidade temos 
que

$$
\begin{aligned}
& U_{\beta, \max }(\beta, \gamma,-y)=X^{\top}(-y) \Phi^{-1} T(-y)\left(\mathcal{I}-\mathcal{Z}^{\dagger}(-y)\right) \iota \\
& =-X^{\top}(y) \Phi^{-1}\left(\mathcal{I}-\mathcal{Z}^{*}(y)\right) \iota \\
& =U_{\beta, \min }(\beta, \gamma, y) \\
& U_{\gamma, \max }(\beta, \gamma,-y)=Z^{\top} \Phi^{-1} H\left(-\mathcal{I}+\mathcal{Z}(-y)-\mathcal{Z}(-y) \mathcal{Z}^{\dagger}(-y)\right) \iota \\
& =Z^{\top} \Phi^{-1} H\left(-\mathcal{I}-\mathcal{Z}(y)+\mathcal{Z}(y) \mathcal{Z}^{*}(y)\right) \iota \\
& =U_{\gamma, \min }(\beta, \gamma, y) \\
& J_{\beta \beta \max }(-y)=X^{\top}(-y) \Phi^{-1} T(-y)\left(\mathcal{Z}^{\dagger}(-y) \Phi^{-1}+\left(\mathcal{I}-\mathcal{Z}^{\dagger}(-y)\right) S T(-y)\right) T(-y) X(-y) \\
& -\left[\iota^{\top}\left(\mathcal{I}-\mathcal{Z}^{\dagger}(-y)\right) T(-y) \Phi^{-1}\right][\dot{X}(-y)] \\
& =-X^{\top}(y) \Phi^{-1} T(-y)\left(\mathcal{Z}^{*}(y) \Phi^{-1}+\left(\mathcal{I}-\mathcal{Z}^{*}(y)\right) S T(-y)\right) T(-y)(-X(y)) \\
& -\left[\iota^{\top}\left(\mathcal{I}-\mathcal{Z}^{*}(y)\right) T(-y) \Phi^{-1}\right][-\dot{X}(y)] \\
& =X^{\top}(y) \Phi^{-1}\left(\mathcal{Z}^{*}(y) \Phi^{-1}+\left(\mathcal{I}-\mathcal{Z}^{*}(y)\right) S\right) X(y) \\
& +\left[\iota^{\top}\left(\mathcal{I}-\mathcal{Z}^{*}(y)\right) \Phi^{-1}\right][\dot{X}(y)] \\
& =J_{\beta \beta \min }(y) \\
& J_{\beta \gamma \max }(-y)=J_{\gamma \beta \max }^{\top}(-y)=X^{\top}(-y) \Phi^{-1} T(-y)\left(\mathcal{I}-\mathcal{Z}^{\dagger}(-y)+\mathcal{Z}(-y) \mathcal{Z}^{\dagger}(-y)\right) H \Phi^{-1} Z \\
& =-X^{\top}(y) \Phi^{-1} T(-y)\left(\mathcal{I}-\mathcal{Z}^{*}(y)-\mathcal{Z}(y) \mathcal{Z}^{*}(y)\right) H \Phi^{-1} Z \\
& =J_{\beta \gamma \min }(y)=J_{\gamma \beta \min }^{\top}(y) \\
& J_{\gamma \gamma \max }(-y)=Z^{\top} \Phi^{-1} H\left(\left(-\mathcal{I}+2 \mathcal{Z}(-y)-2 \mathcal{Z}(-y) \mathcal{Z}^{\dagger}(-y)+\mathcal{Z}^{2}(-y) \mathcal{Z}^{\dagger}(-y)\right) \Phi^{-1}\right. \\
& \left.+\left(-\mathcal{I}+\mathcal{Z}(-y)-\mathcal{Z}(-y) \mathcal{Z}^{\dagger}(-y)\right) Q H\right) H Z \\
& +\left[\iota^{\top}\left(\mathcal{I}-\mathcal{Z}(-y)+\mathcal{Z}(-y) \mathcal{Z}^{\dagger}(-y)\right) H \Phi^{-1}\right][\dot{Z}] \\
& =Z^{\top} \Phi^{-1} H\left(\left(-\mathcal{I}-2 \mathcal{Z}(y)+2 \mathcal{Z}(y) \mathcal{Z}^{*}(y)+\mathcal{Z}^{2}(y) \mathcal{Z}^{*}(y)\right) \Phi^{-1}\right. \\
& \left.+\left(-\mathcal{I}-\mathcal{Z}(y)+\mathcal{Z}(y) \mathcal{Z}^{*}(y)\right) Q H\right) H Z \\
& +\left[\iota^{\top}\left(\mathcal{I}+\mathcal{Z}(y)-\mathcal{Z}(y) \mathcal{Z}^{*}(y)\right) H \Phi^{-1}\right][\dot{Z}] \\
& =J_{\gamma \gamma \min }(y) \text {, } \\
& I_{\beta \beta \max }(-y)=X(-y)^{\top} \Phi^{-1} T(-y)^{2} \Phi^{-1} X(-y)=-X(y)^{\top} \Phi^{-1} T(-y)^{2} \Phi^{-1}(-X(y))=I_{\beta \beta \min }(y)
\end{aligned}
$$




$$
\begin{aligned}
I_{\beta \gamma, \text { max }}(-y)=I_{\gamma \beta, \max }^{\top}(-y) & =(\mathcal{E}-1) X^{\top}(-y) \Phi^{-1} T(-y) H \Phi^{-1} Z \\
& =-(\mathcal{E}-1) X^{\top}(y) \Phi^{-1} H \Phi^{-1} Z \\
& =I_{\beta \gamma, \min }(y)=I_{\gamma \beta, \min }^{\top}(-y) .
\end{aligned}
$$

$\mathrm{e}$

$$
I_{\gamma \gamma, \max }(-y)=\left(1+\Gamma^{(2)}(2)\right) Z^{\top} \Phi^{-1} H^{2} \Phi^{-1} Z=I_{\gamma \gamma, \min }(y),
$$

em que indicamos como função de $y$ somente as quantidades que se alteram quando calculadas em $-y$.

Então podemos concluir que, se definirmos o preditor $\eta^{*}(\beta,-\mu)=-\eta(\beta, \mu)$, então $w_{\max }(-y)=$ $w_{\min }(y), W_{\max }(-y)=W_{\min }(y), S_{R, \max }(-y)=S_{R, \min }(y), S_{T, \max }(-y)=S_{T, \min }(y)$ e $w_{\max }^{*}(-y)=$ $w_{\text {min }}^{*}(y)$.

Assim, se $y_{t} \sim V E_{\min }\left(\mu_{t}, \sigma_{t}\right)$ e $g(\mu)=\eta(\beta)$ então $-y_{t}, t=1, \ldots, n$, tem distribuição valor extremo máximo com parâmetros $-\mu_{t}$ e $\sigma_{t}\left(-y_{t} \sim V E_{\max }\left(-\mu_{t}, \sigma_{t}\right)\right)$, e podemos obter as estatísticas $w, W, S_{R}$, $S_{T}$ e $w^{*}$, para o modelo (2.28)-(2.2)-(2.3), calculando as respectivas fórmulas, apresentadas na Seção 2.2, para $-y$, função de ligação do parâmetro de locação $g^{*}=i d$ e preditor do parâmetro de locação $\eta^{*}(\beta)=$ $\left(-g^{-1} \circ \eta\right)(\beta)$. Como caso particular, para o modelo linear $\eta=X \beta$ e função de ligação identidade, definimos $\eta^{*}(\beta)=-X \beta=(-X) \beta$.

\subsection{Simulações - valor extremo máximo}

Apresentamos a seguir o comportamento do teste da razão de verossimilhanças $(w)$, de Wald $(W)$, escore $\left(S_{R}\right)$, gradiente $\left(S_{T}\right)$ e o teste da razão de verossimilhanças ajustada $\left(w^{*}\right)$ para amostras pequenas e de tamanho moderado, através dos resultados das simulações de Monte Carlo. Os parâmetros são estimados através da maximização do logaritmo da função de verossimilhança utilizando o método de otimização não-linear quasi-Newton BFGS com primeiras derivadas analíticas desenvolvido por Broyden, Fletcher, Goldfarf \& Shanno (veja, por exemplo, Press et al., 1992 ), implementado na linguagem de programação Ox na função MaxBFGS.

Nas Seções 2.4.1, 2.4.2, 2.4.3 e 2.4.4 consideramos os modelos de regressão valor extremo máximo linear e não linear com dispersão constante e variável para cada um deles. O número de réplicas de Monte Carlo usado para comparação de tamanho dos testes foi 10.000 e os níveis nominais dos testes foram $\alpha=10 \%, 5 \%$ e $1 \%$. Se número de elementos da amostra é pequeno ou moderado, os resultados da simulação mostram que os testes têm tamanhos distintos quando a distribuição assintótica $\chi^{2}$ é usada como aproximação. Para avaliar o poder destes testes, deve-se garantir que tenham o tamanho correto sob a hipótese nula. A fim de contornar esta dificuldade, foram simuladas 500.000 amostras de Monte Carlo sob cada hipótese nula estudada, para obter a distribuição empírica das estatísticas e estimar o valor crítico exato para cada teste para o nível nominal escolhido. Foram usados como valores críticos exatos, para avaliar o poder dos testes, os quantis amostrais de ordem $90 \%$ das distribuições empíricas das estatísticas. Todas as simulações foram realizadas com a linguagem de programação Ox (Doornik, 2009). 


\subsubsection{Modelo de regressão valor extremo máximo linear com dispersão constante}

Considera-se o modelo (2.1) com parâmetro de dispersão constante e com componente sistemático da localização dado por

$$
\mu_{t}=\beta_{1}+\beta_{2} x_{t 2}+\beta_{3} x_{t 3}+\beta_{4} x_{t 4}+\beta_{5} x_{t 5},
$$

o qual denominamos de 'modelo 1'. Consideram-se três hipóteses nulas distintas, a saber $\mathcal{H}_{0}: \beta_{2}=0$ $(r=1), \mathcal{H}_{0}: \beta_{2}=\beta_{3}=0(r=2)$ e $\mathcal{H}_{0}: \beta_{2}=\beta_{3}=\beta_{4}=0(r=3)$, a serem testadas contra uma alternativa bilateral. Para o primeiro caso, assume-se $\beta_{1}=1, \beta_{2}=0, \beta_{3}=1, \beta_{4}=6$ e $\beta_{5}=-3$. Para o segundo caso, $\beta_{1}=1, \beta_{2}=\beta_{3}=0, \beta_{4}=6$ e $\beta_{5}=-3$. Finalmente, para o terceiro caso $\beta_{1}=1$, $\beta_{2}=\beta_{3}=\beta_{4}=0$ e $\beta_{5}=-3$. Nas três configurações fizemos $\sigma=0,1$. Consideramos apenas um valor para $\sigma$ pois as distribuições das estatísticas, nas condições acima, não dependem do valor de $\sigma$, conforme demonstrado no Apêndice D. Os valores das covariadas foram obtidos aleatoriamente de uma distribuição $\mathcal{U}(-0,5,0,5)$. Os tamanhos das amostras utilizados são 15, 20, 30 e 40. Os resultados são resumidos nas Tabelas 2.1 e 2.2 e nas Figuras 2.1 e 2.2.

Tabela 2.1: Taxas de rejeição nula (\%); modelo 1

\begin{tabular}{|c|c|c|c|c|c|c|c|c|c|c|c|c|c|c|c|c|}
\hline \multirow[b]{2}{*}{$r$} & \multirow[b]{2}{*}{$n$} & \multicolumn{5}{|c|}{$\alpha=10 \%$} & \multicolumn{5}{|c|}{$\alpha=5 \%$} & \multicolumn{5}{|c|}{$\alpha=1 \%$} \\
\hline & & $w$ & $W$ & $S_{R}$ & $S_{T}$ & $w^{*}$ & $w$ & $W$ & $S_{R}$ & $S_{T}$ & $w^{*}$ & $w$ & $W$ & $S_{R}$ & $S_{T}$ & $w^{*}$ \\
\hline \multirow{4}{*}{1} & 15 & 19,3 & 28,2 & 11,5 & 16,9 & 10,4 & 11,6 & 20,8 & 5,8 & 9,0 & 5,0 & 4,0 & 11,1 & 1,1 & 1,8 & 1,0 \\
\hline & 20 & 16,8 & 22,1 & 11,3 & 15,0 & 9,9 & 10,0 & 15,6 & 5,4 & 7,8 & 4,8 & 3,0 & 7,0 & 0,9 & 1,5 & 0,9 \\
\hline & 30 & 14,9 & 18,9 & 10,9 & 13,8 & 10,6 & 8,5 & 12,0 & 5,3 & 7,1 & 5,4 & 2,4 & 4,6 & 0,9 & 1,4 & 1,2 \\
\hline & 40 & 13,0 & 15,7 & 10,3 & 12,1 & 10,1 & 7,0 & 9,5 & 4,9 & 6,2 & 5,1 & 2,0 & 3,5 & 0,9 & 1,3 & 1,1 \\
\hline \multirow{4}{*}{2} & 15 & 22,9 & 36,5 & 12,7 & 16,4 & 7,7 & 14,3 & 28,7 & 6,2 & 7,9 & 3,5 & 4,8 & 17,1 & 1,1 & 0,8 & 0,6 \\
\hline & 20 & 18,8 & 28,8 & 11,6 & 14,1 & 9,2 & 11,4 & 20,9 & 5,4 & 7,2 & 4,6 & 3,8 & 10,5 & 0,9 & 0,8 & 0,8 \\
\hline & 30 & 16,0 & 23,4 & 10,8 & 13,0 & 10,2 & 9,2 & 15,7 & 5,2 & 6,6 & 5,2 & 2,3 & 6,7 & 0,8 & 0,9 & 0,9 \\
\hline & 40 & 13,9 & 19,0 & 10,2 & 11,7 & 9,8 & 7,6 & 12,0 & 5,0 & 6,1 & 5,2 & 1,9 & 4,5 & 0,7 & 0,9 & 0,9 \\
\hline \multirow{4}{*}{3} & 15 & 23,4 & 42,9 & 9,1 & 12,6 & 6,8 & 14,9 & 34,2 & 3,4 & 4,8 & 3,0 & 5,1 & 21,2 & 0,2 & 0,2 & 0,5 \\
\hline & 20 & 19,8 & 33,8 & 9,8 & 12,3 & 8,7 & 12,1 & 25,1 & 4,4 & 5,4 & 4,4 & 3,8 & 14,0 & 0,5 & 0,4 & 0,8 \\
\hline & 30 & 16,6 & 26,4 & 10,1 & 12,1 & 9,9 & 9,5 & 18,2 & 4,7 & 5,4 & 4,9 & 2,5 & 8,6 & 0,6 & 0,6 & 0,9 \\
\hline & 40 & 14,7 & 21,4 & 10,2 & 11,3 & 10,3 & 8,3 & 14,0 & 4,6 & 5,5 & 5,2 & 2,1 & 5,8 & 0,7 & 0,7 & 0,9 \\
\hline
\end{tabular}

Algumas conclusões interessantes podem ser tiradas da Tabela 2.1. A primeira é que o teste da razão de verossimilhanças e o teste de Wald são notadamente liberais para amostras pequenas. Por exemplo, para $n=15, r=1$ e $\alpha=5 \%$, a taxa de rejeição nula é $11,6 \%$ e $20,8 \%$ para o teste da razão de verossimilhanças e o teste de Wald, respectivamente. O teste gradiente pode ser liberal, mas bem menos que os dois testes mencionados acima. O teste escore é menos liberal e pode ser conservativo em alguns casos. $\mathrm{O}$ teste da razão de verossimilhanças ajustado se comporta melhor que todos os outros em relação ao erro do tipo I na maioria dos casos. Para o caso mencionado acima, a taxa de rejeição nula do teste ajustado é 5,0\% $\left(w^{*}\right)$. Chamamos de discrepância relativa de quantis a diferença entre o quantil exato (estimado por simulação) e o quantil assintótico, dividido pelo quantil assintótico. Quanto mais próxima de zero a discrepância de quantis, melhor é a proximação da distribuição nula exata da estatística do teste e da distribuição assíntota $\chi^{2}$ (ver Ferrari \& Cysneiros, 2008, p. 3050). A Figura 2.1 apresenta gráficos de discrepâncias relativas dos quantis das diferentes estatísticas para teste das hipóteses $\mathcal{H}_{0}: \beta_{2}=0(r=1), \mathcal{H}_{0}: \beta_{2}=\beta_{3}=0(r=2)$ 

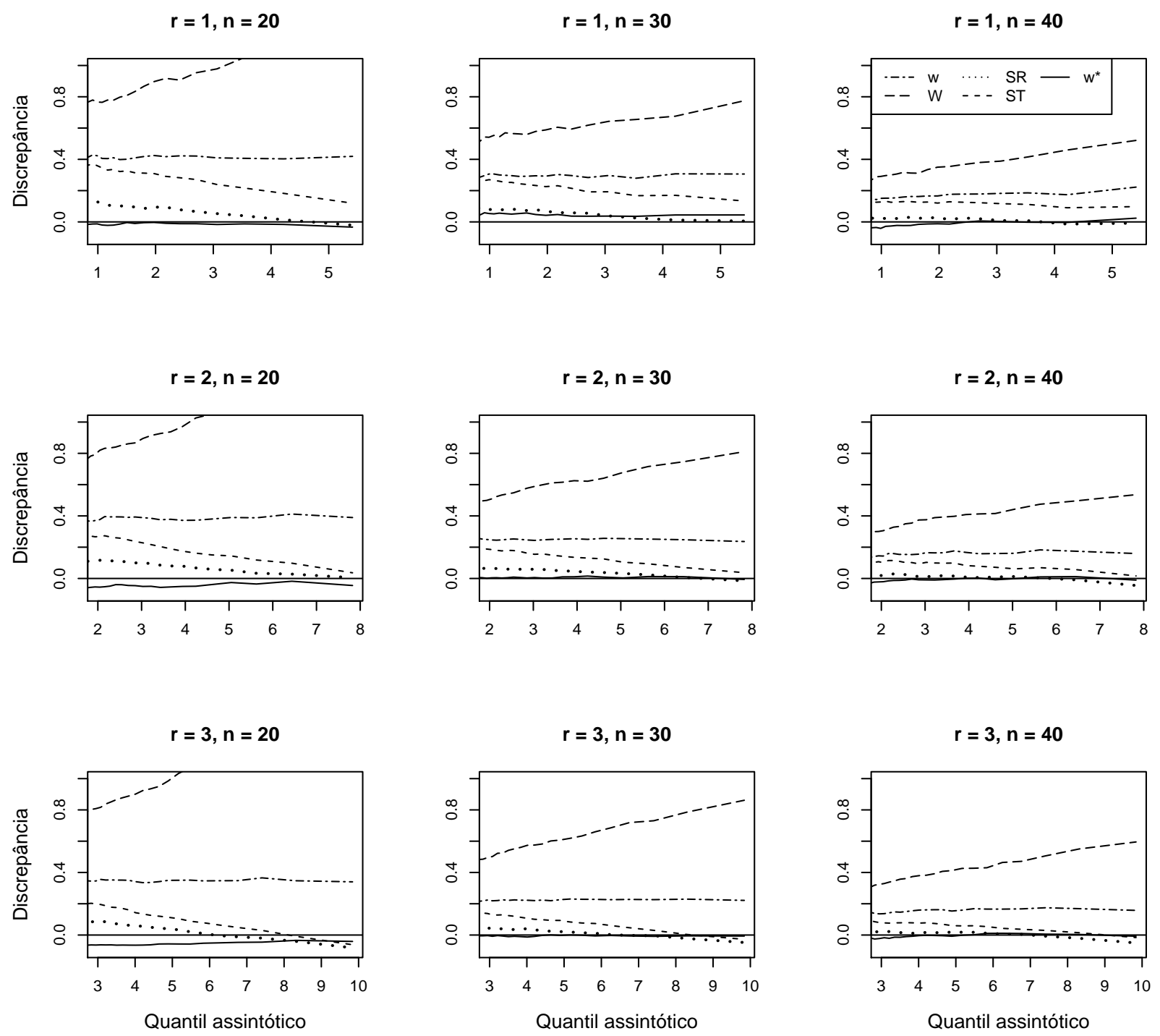

Figura 2.1: Discrepâncias relativas de quantis; modelo 1.

e $\mathcal{H}_{0}: \beta_{2}=\beta_{3}=\beta_{4}=0(r=3)$. Os quantis assintóticos são obtidos de uma distribuição $\chi_{1}^{2}, \chi_{2}^{2}$ e $\chi_{3}^{2}$, quando $r=1, r=2$ e $r=3$, respectivamente. As figuras confirmam a tendência do teste da razão de verossimilhanças usual e Wald de rejeitar a hipótese nula com frequência maior do que o esperado, baseado no nível nominal. O teste de Wald e da razão de verossimilhanças são muito liberais, isto é, apresentam taxas de rejeição nula empíricas bem acima dos níveis nominais. Nota-se que a distribuição da estatística ajustada $w^{*}$ é muito mais próxima da distribuição de referência do que a distribuição de $w$. A melhor concordância entre os quantis verdadeiros e assintóticos é alcançada por $w^{*}$. A distribuição empírica da estatística ajustada está muito próxima das respectivas distribuições limite mesmo quando a amostra é pequena.

Para comparação de poder, as taxas de rejeição foram obtidas sob a hipótese alternativa $\beta_{2}=\epsilon(r=1)$, $\beta_{2}=\beta_{3}=\epsilon(r=2)$ e $\beta_{2}=\beta_{3}=\beta_{4}=\epsilon(r=3)$, para diferentes valores de $\epsilon$ com base em 10.000 amostras de Monte Carlo. A Tabela 2.2 apresenta taxas de rejeição não nula (poder) dos testes ajustados das 
Tabela 2.2: Taxas de rejeição não nula (\%); modelo $1, n=30, \alpha=10 \%$

\begin{tabular}{c|c|cccccccccccc}
\hline \hline$r$ & $\epsilon$ & $-0,3$ & $-0,2$ & $-0,1$ & $-0,05$ & $-0,025$ & $-0,01$ & 0,01 & 0,025 & 0,05 & 0,1 & 0,2 & 0,3 \\
\hline & $w$ & 98,2 & 81,4 & 35,6 & 16,6 & 11,7 & 10,5 & 11,0 & 12,6 & 18,1 & 37,4 & 82,2 & 98,1 \\
& $W$ & 98,4 & 82,1 & 35,9 & 17,1 & 11,8 & 10,8 & 10,9 & 12,6 & 17,8 & 36,3 & 82,0 & 98,3 \\
1 & $S_{R}$ & 97,3 & 78,9 & 33,5 & 16,0 & 11,2 & 10,2 & 11,1 & 13,1 & 18,7 & 38,5 & 81,9 & 97,8 \\
& $S_{T}$ & 98,2 & 81,1 & 35,3 & 16,4 & 11,5 & 10,4 & 11,0 & 12,8 & 18,4 & 37,8 & 82,6 & 98,2 \\
& $w^{*}$ & 98,4 & 81,8 & 36,2 & 16,9 & 11,7 & 10,6 & 10,8 & 12,7 & 18,0 & 36,8 & 82,2 & 98,2 \\
\hline \multirow{4}{*}{2} & $w$ & 99,8 & 92,5 & 44,1 & 18,5 & 12,1 & 10,4 & 10,8 & 12,9 & 20,4 & 46,9 & 93,4 & 99,8 \\
& $W$ & 99,9 & 92,6 & 42,6 & 17,9 & 12,2 & 10,4 & 10,6 & 12,8 & 19,2 & 45,1 & 93,1 & 99,9 \\
& $S_{R}$ & 99,5 & 90,1 & 42,1 & 17,4 & 11,4 & 10,1 & 10,8 & 13,3 & 20,6 & 46,3 & 91,0 & 99,3 \\
& $S_{T}$ & 99,8 & 92,6 & 43,8 & 18,3 & 11,8 & 10,4 & 10,7 & 13,0 & 20,5 & 46,9 & 93,5 & 99,8 \\
& $w^{*}$ & 99,8 & 93,0 & 45,3 & 19,1 & 12,5 & 10,7 & 10,5 & 12,3 & 19,5 & 45,5 & 92,9 & 99,8 \\
\hline & $w$ & 100,0 & 98,9 & 61,3 & 23,4 & 13,1 & 10,9 & 11,3 & 14,1 & 25,3 & 62,7 & 99,0 & 100,0 \\
& $W$ & 100,0 & 99,0 & 58,3 & 22,1 & 13,1 & 10,8 & 11,1 & 13,5 & 23,2 & 59,5 & 99,1 & 100,0 \\
3 & $S_{R}$ & 99,9 & 97,6 & 58,7 & 22,6 & 12,7 & 10,4 & 11,2 & 14,5 & 26,1 & 61,4 & 97,2 & 99,9 \\
& $S_{T}$ & 100,0 & 99,0 & 61,0 & 23,1 & 13,0 & 10,8 & 11,3 & 14,0 & 25,4 & 62,8 & 99,0 & 100,0 \\
& $w^{*}$ & 100,0 & 98,9 & 61,2 & 23,8 & 13,5 & 11,0 & 11,1 & 13,5 & 24,7 & 61,9 & 98,9 & 100,0 \\
\hline \hline
\end{tabular}

três hipóteses nulas para o modelo $1 \operatorname{com} n=30$ e $\alpha=10 \%$. Os poderes dos testes são similares. Em geral não há evidência de que um dos testes seja mais poderoso do que os outros. Podemos confirmar a semelhança dos testes em relação ao poder pela Figura 2.2 observando que as curvas praticamente coincidem.

\subsubsection{Modelo de regressão valor extremo máximo linear com dispersão variável}

\subsubsection{Teste dos coeficientes da regressão do parâmetro de localização}

Considera-se o modelo (2.1) com os componentes sistemáticos para a localização e para a dispersão dados por

$$
\begin{gathered}
\mu_{t}=\beta_{1}+\beta_{2} x_{t 2}+\beta_{3} x_{t 3}+\beta_{4} x_{t 4} \\
\ln \left(\sigma_{t}\right)=\gamma_{1}+\gamma_{2} z_{t 2}+\gamma_{3} z_{t 3}+\gamma_{4} z_{t 4},
\end{gathered}
$$

o qual denominamos de 'modelo 2'.

Consideram-se três hipóteses nulas distintas, a saber: $\mathcal{H}_{0}: \beta_{4}=0(r=1), \mathcal{H}_{0}: \beta_{3}=\beta_{4}=0(r=2)$ e $\mathcal{H}_{0}: \beta_{2}=\beta_{3}=\beta_{4}=0(r=3)$, a serem testadas contra uma alternativa bilateral. Para o primeiro caso, assume-se que $\beta_{1}=1, \beta_{2}=1, \beta_{3}=6$ e $\beta_{4}=0$. Para o segundo caso, $\beta_{1}=1, \beta_{2}=1$ e $\beta_{3}=\beta_{4}=0$. Finalmente, para o terceiro caso $\beta_{1}=1$ e $\beta_{2}=\beta_{3}=\beta_{4}=0$. Em todas as situações consideradas acima, assume-se que $\gamma_{1}=\ln (0,1)=-2,30259, \gamma_{2}=-2, \gamma_{3}=-2$ e $\gamma_{4}=0,1$. Os valores das covariadas foram obtidos aleatoriamente de uma distribuição $\mathcal{U}(-0,5,0,5)$. Os tamanhos das amostras utilizados são 40, 50, 60 e 70. Os resultados são resumidos nas Tabelas 2.3 e 2.4 e na Figura 2.3.

Observa-se, por exemplo, na Tabela 2.3 que para $n=50, r=1$ e $\alpha=10 \%$, a taxa de rejeição nula do teste da razão de verossimilhanças é $15,9 \%$, enquanto a do teste ajustado é $10,1 \%$. Para $r=1$ aa taxas de rejeição nula do teste escore estão muito próximas do nível nominal. Para $r=3$ e $\alpha=5 \%$, a taxa de 


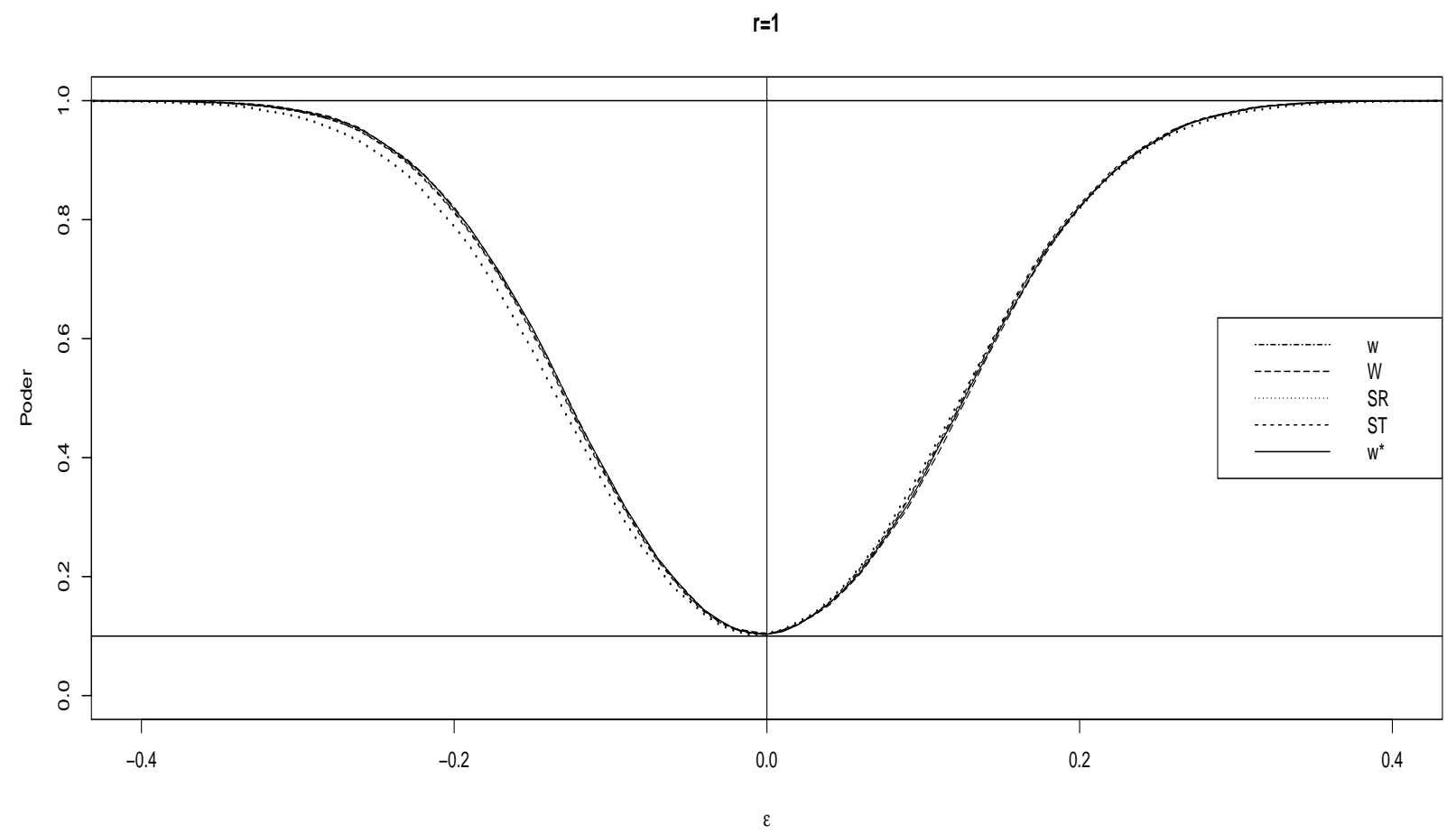

Figura 2.2: Poder dos testes; modelo $1, r=1, n=30$.

Tabela 2.3: Taxas de rejeição nula (\%); modelo 2, localização

\begin{tabular}{c|c|ccccc|cccccc|cccc}
\hline \hline \multirow{7}{*}{$r$} & & \multicolumn{7}{|c|}{$\alpha=10 \%$} & \multicolumn{5}{c|}{$\alpha=5 \%$} & \multicolumn{5}{c}{$\alpha=1 \%$} \\
\cline { 3 - 15 } & $w$ & $W$ & $S_{R}$ & $S_{T}$ & $w^{*}$ & $w$ & $W$ & $S_{R}$ & $S_{T}$ & $w^{*}$ & $w$ & $W$ & $S_{R}$ & $S_{T}$ & $w^{*}$ \\
\hline 1 & 40 & 17,5 & 24,2 & 10,8 & 15,3 & 11,1 & 10,4 & 16,8 & 5,7 & 8,4 & 6,1 & 3,4 & 8,0 & 1,0 & 1,7 & 1,6 \\
& 50 & 15,9 & 20,6 & 10,8 & 14,1 & 10,1 & 8,9 & 13,4 & 5,2 & 7,2 & 5,3 & 2,3 & 5,4 & 0,8 & 1,4 & 1,1 \\
& 60 & 15,9 & 21,9 & 10,6 & 13,9 & 10,7 & 9,3 & 15,3 & 5,1 & 7,0 & 5,7 & 2,4 & 7,0 & 0,8 & 1,2 & 1,2 \\
& 70 & 14,8 & 19,9 & 10,0 & 12,9 & 10,5 & 8,4 & 13,1 & 5,0 & 6,6 & 5,4 & 2,0 & 5,6 & 0,7 & 1,1 & 1,2 \\
\hline \multirow{2}{*}{2} & 40 & 21,7 & 36,9 & 8,3 & 13,7 & 11,8 & 13,7 & 28,6 & 3,7 & 6,4 & 6,6 & 4,5 & 16,6 & 0,6 & 0,9 & 2,0 \\
& 50 & 18,6 & 29,4 & 9,0 & 13,5 & 10,9 & 11,0 & 21,4 & 3,9 & 6,3 & 5,7 & 3,2 & 11,1 & 0,5 & 0,9 & 1,4 \\
& 60 & 17,0 & 26,9 & 9,0 & 12,8 & 10,6 & 9,7 & 19,6 & 3,9 & 5,9 & 5,4 & 2,9 & 9,9 & 0,5 & 0,9 & 1,3 \\
& 70 & 15,8 & 24,5 & 8,8 & 12,4 & 10,3 & 9,0 & 17,1 & 4,0 & 5,6 & 5,2 & 2,3 & 8,0 & 0,6 & 0,9 & 1,2 \\
\hline 3 & 40 & 23,1 & 43,2 & 7,9 & 12,1 & 11,9 & 14,1 & 34,6 & 3,3 & 5,1 & 6,8 & 4,7 & 21,7 & 0,3 & 0,6 & 2,1 \\
& 50 & 19,3 & 34,0 & 8,1 & 12,1 & 10,9 & 11,5 & 25,9 & 3,5 & 5,2 & 5,6 & 3,4 & 14,4 & 0,4 & 0,7 & 1,4 \\
& 60 & 17,2 & 30,4 & 8,1 & 11,4 & 10,1 & 9,9 & 22,3 & 3,6 & 5,4 & 5,2 & 2,8 & 11,6 & 0,5 & 0,8 & 1,3 \\
& 70 & 16,4 & 27,3 & 8,3 & 11,4 & 10,2 & 9,4 & 19,8 & 3,6 & 5,0 & 5,0 & 2,3 & 9,2 & 0,6 & 0,7 & 1,1 \\
\hline \hline
\end{tabular}

rejeição nula do teste gradiente está muito próxima do nível nominal, mesmo para $n=40$.

A Figura 2.3 apresenta gráficos de discrepâncias relativas dos quantis das diferentes estatísticas para teste das hipóteses $\mathcal{H}_{0}: \beta_{2}=0(r=1), \mathcal{H}_{0}: \beta_{2}=\beta_{3}=0(r=2)$ e $\mathcal{H}_{0}: \beta_{2}=\beta_{3}=\beta_{4}=0(r=3)$. Os quantis assintóticos são obtidos de uma distribuição $\chi_{1}^{2}, \chi_{2}^{2}$ e $\chi_{3}^{2}$, respectivamente. A estatística da razão de verossimilhanças usual apresenta o mesmo comportamento da situação anterior, rejeitando a hipótese nula com mais frequência do que o esperado, baseado no nível nominal. Sua distribuição difere visivelmente da distribuição de referência enquanto a distribuição da estatística ajustada é muito mais próxima desta. 

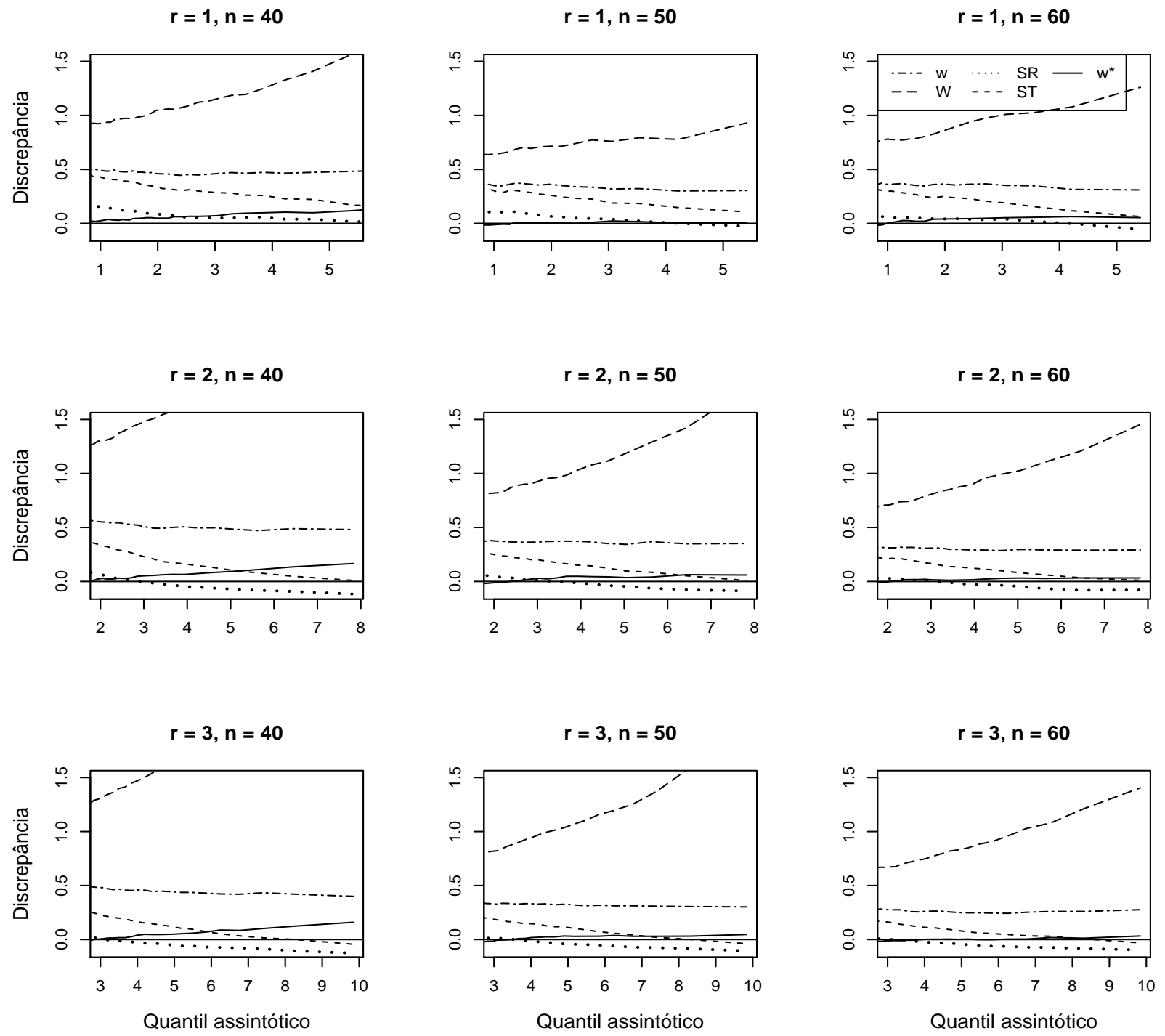

Figura 2.3: Discrepâncias relativas de quantis; modelo 2, localização.

A melhor concordância entre os quantis verdadeiros e assintóticos é alcançada por $w^{*}$. A estatística escore também apresenta um bom desempenho tendo sua distribuição próxima da assíntota para os três tamanhos de amostra apresentados, mas tende a ser conservadora. A estatística gradiente tem um bom desempenho para quantis grandes. Nas três situações as distribuições empíricas da estatística ajustada estão muito próximas das respectivas distribuições limite mesmo quando a amostra é de tamanho moderado, isto é, $n=50$. .

A Tabela 2.4 apresenta taxas de rejeição não nula (poder) dos testes para o modelo 2 com $n=40$ e $\alpha=10 \%$. Aqui, as taxas de rejeição foram obtidas sob as hipóteses alternativas $\beta_{4}=\epsilon(r=1)$, $\beta_{3}=\beta_{4}=\epsilon(r=2)$ e $\beta_{2}=\beta_{3}=\beta_{4}=\epsilon(r=3)$ para diferentes valores de $\epsilon$. Os poderes dos testes são similares, não havendo um teste que tenha melhor desempenho em todas as situações. Não há diferença relevante do poder dos testes do modelo 2 em comparação ao poder dos testes do modelo 1. 
Tabela 2.4: Taxas de rejeição não nula (\%); modelo 2 , localização, $n=40, \alpha=10 \%$

\begin{tabular}{c|c|cccccccccc}
\hline \hline$r$ & $\epsilon$ & $-0,30$ & $-0,20$ & $-0,10$ & $-0,05$ & $-0,03$ & 0,03 & 0,05 & 0,10 & 0,20 & 0,30 \\
\hline \multirow{4}{*}{1} & $w$ & 98,7 & 85,3 & 39,2 & 17,8 & 12,7 & 13,0 & 18,3 & 39,8 & 84,3 & 98,5 \\
& $W$ & 99,0 & 85,1 & 38,6 & 17,9 & 13,1 & 12,2 & 16,9 & 37,4 & 82,9 & 98,5 \\
& $S_{R}$ & 96,7 & 80,9 & 36,1 & 16,0 & 11,7 & 14,0 & 20,3 & 42,4 & 84,8 & 98,0 \\
& $S_{T}$ & 98,7 & 85,3 & 39,2 & 17,7 & 12,5 & 13,1 & 18,5 & 40,5 & 85,0 & 98,7 \\
& $w^{*}$ & 98,8 & 84,4 & 37,4 & 16,5 & 12,1 & 12,9 & 18,2 & 39,7 & 84,3 & 98,6 \\
\hline \multirow{4}{*}{2} & $w$ & 99,9 & 96,6 & 53,1 & 19,9 & 12,7 & 15,6 & 24,3 & 55,7 & 95,2 & 99,8 \\
& $W$ & 99,9 & 94,8 & 44,7 & 16,2 & 11,2 & 16,1 & 24,0 & 54,7 & 95,5 & 99,9 \\
& $S_{R}$ & 99,4 & 95,1 & 54,4 & 22,0 & 13,5 & 14,6 & 21,7 & 48,7 & 88,8 & 98,6 \\
& $S_{T}$ & 99,9 & 97,1 & 55,6 & 21,6 & 13,6 & 14,6 & 22,3 & 53,3 & 95,1 & 99,8 \\
& $w^{*}$ & 99,9 & 97,4 & 56,6 & 22,4 & 14,1 & 13,5 & 20,2 & 49,4 & 93,7 & 99,7 \\
\hline \multirow{4}{*}{3} & $w$ & 100,0 & 99,9 & 90,9 & 41,0 & 19,4 & 21,3 & 39,1 & 85,4 & 99,9 & 100,0 \\
& $W$ & 100,0 & 100,0 & 80,1 & 26,7 & 13,9 & 22,3 & 39,6 & 85,4 & 99,9 & 100,0 \\
& $S_{R}$ & 100,0 & 99,8 & 92,1 & 49,5 & 25,2 & 15,7 & 28,0 & 66,4 & 97,8 & 99,8 \\
& $S_{T}$ & 100,0 & 100,0 & 90,9 & 42,9 & 21,3 & 18,4 & 35,4 & 82,8 & 99,9 & 100,0 \\
& $w^{*}$ & 100,0 & 99,9 & 91,0 & 42,5 & 21,1 & 17,6 & 33,4 & 80,4 & 99,9 & 100,0 \\
\hline \hline
\end{tabular}

\subsubsection{Teste dos coeficientes da regressão do parâmetro de dispersão}

Considera-se o modelo (2.1) com os componentes sistemáticos para a localização e para dispersão dados por

$$
\begin{gathered}
\mu_{t}=\beta_{1}+\beta_{2} x_{t 2}+\beta_{3} x_{t 3}+\beta_{4} x_{t 4}, \\
\ln \left(\sigma_{t}\right)=\gamma_{1}+\sum_{j=2}^{m} \gamma_{j} z_{t j}, \quad m=2,3,4,
\end{gathered}
$$

o qual denominamos de 'modelo 2.1'.

Consideram-se as hipóteses nulas $\mathcal{H}_{0}: \gamma_{2}=\ldots=\gamma_{m}=0$, para $m=2,3$, 4 e $r=1,2,3$, respectivamente, a serem testadas contra alternativas bilaterais. Assume-se $\beta_{1}=1, \beta_{2}=1, \beta_{3}=6, \beta_{4}=1$, $\gamma_{1}=-2,30259$ e $\gamma_{2}=0, \gamma_{2}=\gamma_{3}=0$ e $\gamma_{2}=\gamma_{3}=\gamma_{4}=0$, para $r=1,2,3$, respectivamente. Os valores das covariadas foram obtidos aleatoriamente de uma distribuição $\mathcal{U}(-0,5,0,5)$. Os tamanhos das amostras utilizados são 40, 50, 60, 70, 80 e 90.

Observa-se na Tabela 2.5 que há um ganho não desprezível, nas duas configurações testadas, em relação à probabilidade do erro de tipo I quando se utiliza o teste ajustado, pois é o que se aproxima mais rápido do nível nominal conforme aumenta o tamanho da amostra. Em todas as situações o teste ajustado é o que tem o tamanho mais próximo do nível nominal. O teste escore é conservativo, mas o tamanho está próximo do nível nominal mesmo para amostras pequenas. Nota-se que à medida em que o número de observações cresce há uma melhora significativa em relação à proximidade entre a distribuição de referência e as distribuições das estatísticas, isto é, as distorções de tamanho dos testes são reduzidas, em especial no que se refere ao teste ajustado $w^{*}$. Por exemplo, para $r=1, n=70$ e $\alpha=10 \%$, a probabilidade do erro de tipo I do teste da razão de verossimilhanças usual é $12 \%$, enquanto a do teste ajustado é 10,3\%. Como 
Tabela 2.5: Taxas de rejeição nula (\%); modelo 2.1, dispersão

\begin{tabular}{|c|c|c|c|c|c|c|c|c|c|c|c|c|c|c|c|c|}
\hline \multirow[b]{2}{*}{$r$} & \multirow[b]{2}{*}{$n$} & \multicolumn{5}{|c|}{$\alpha=10 \%$} & \multicolumn{5}{|c|}{$\alpha=5 \%$} & \multicolumn{5}{|c|}{$\alpha=1 \%$} \\
\hline & & $w$ & $W$ & $S_{R}$ & $S_{T}$ & $w^{*}$ & $w$ & $W$ & $S_{R}$ & $S_{T}$ & $w^{*}$ & $w$ & $W$ & $S_{R}$ & $S_{T}$ & $w^{*}$ \\
\hline \multirow{6}{*}{1} & 40 & 14,2 & 20,8 & 8,7 & 13,9 & 12,0 & 8,2 & 13,4 & 4,3 & 7,9 & 6,6 & 2,1 & 5,6 & 0,7 & 1,9 & 1,7 \\
\hline & 50 & 12,8 & 17,9 & 8,6 & 12,6 & 11,0 & 7,1 & 11,2 & 4,2 & 6,8 & 5,8 & 2,0 & 4,1 & 0,8 & 1,8 & 1,4 \\
\hline & 60 & 12,9 & 16,8 & 9,1 & 12,8 & 11,1 & 7,1 & 10,6 & 4,4 & 7,0 & 5,8 & 1,7 & 3,4 & 0,8 & 1,6 & 1,2 \\
\hline & 70 & 12,0 & 15,8 & 8,9 & 11,7 & 10,3 & 6,5 & 9,4 & 4,5 & 6,4 & 5,3 & 1,7 & 3,1 & 1,1 & 1,6 & 1,2 \\
\hline & 80 & 11,5 & 14,3 & 8,9 & 11,3 & 10,1 & 5,9 & 8,1 & 4,3 & 5,7 & 4,9 & 1,5 & 2,3 & 0,9 & 1,4 & 1,2 \\
\hline & 90 & 11,8 & 14,1 & 9,4 & 11,7 & 10,6 & 6,3 & 8,3 & 4,8 & 6,2 & 5,4 & 1,4 & 2,2 & 1,0 & 1,3 & 1,1 \\
\hline \multirow{6}{*}{2} & 40 & 16,6 & 27,4 & 9,0 & 16,0 & 13,8 & 10,1 & 19,2 & 4,4 & 9,4 & 7,9 & 2,9 & 8,6 & 0,7 & 2,4 & 2,3 \\
\hline & 50 & 14,8 & 23,1 & 9,0 & 14,5 & 12,3 & 8,6 & 15,6 & 4,3 & 8,1 & 6,6 & 2,2 & 6,2 & 0,8 & 1,9 & 1,6 \\
\hline & 60 & 14,0 & 21,0 & 8,6 & 13,5 & 11,5 & 7,5 & 13,2 & 4,3 & 7,2 & 6,1 & 2,0 & 4,8 & 0,8 & 1,8 & 1,4 \\
\hline & 70 & 13,1 & 19,0 & 9,0 & 12,7 & 11,3 & 7,4 & 11,8 & 4,6 & 7,1 & 6,1 & 1,7 & 4,3 & 0,9 & 1,6 & 1,4 \\
\hline & 80 & 12,0 & 16,9 & 8,7 & 11,9 & 10,5 & 6,6 & 10,2 & 4,5 & 6,5 & 5,5 & 1,5 & 3,1 & 0,9 & 1,4 & 1,2 \\
\hline & 90 & 12,4 & 16,5 & 9,0 & 12,2 & 10,8 & 6,4 & 9,9 & 4,5 & 6,2 & 5,4 & 1,5 & 2,8 & 0,8 & 1,3 & 1,0 \\
\hline \multirow{6}{*}{3} & 40 & 19,3 & 36,8 & 8,4 & 18,3 & 17,9 & 11,9 & 28,2 & 3,7 & 10,9 & 11,0 & 3,5 & 15,4 & 0,7 & 2,7 & 3,9 \\
\hline & 50 & 16,1 & 29,0 & 8,3 & 15,3 & 13,7 & 9,5 & 20,7 & 4,1 & 8,6 & 7,9 & 2,7 & 9,4 & 0,7 & 2,2 & 2,2 \\
\hline & 60 & 14,5 & 24,8 & 8,3 & 14,0 & 12,5 & 8,2 & 16,6 & 4,1 & 7,5 & 7,0 & 2,2 & 7,0 & 0,7 & 1,9 & 1,9 \\
\hline & 70 & 14,0 & 22,4 & 8,5 & 13,4 & 12,1 & 7,7 & 14,4 & 4,4 & 7,3 & 6,5 & 2,0 & 6,0 & 0,8 & 1,7 & 1,6 \\
\hline & 80 & 13,1 & 20,4 & 8,8 & 12,8 & 11,4 & 7,2 & 12,8 & 4,4 & 6,9 & 6,0 & 1,9 & 4,7 & 0,9 & 1,7 & 1,5 \\
\hline & 90 & 12,7 & 18,8 & 8,7 & 12,4 & 10,9 & 6,8 & 11,6 & 4,4 & 6,4 & 5,4 & 1,4 & 3,7 & 0,8 & 1,3 & 1,1 \\
\hline
\end{tabular}

esperado os ajustes são menos necessários para amostras grandes. Todavia, note que não é desprezível o ganho obtido para $n=90$ nas três configurações testadas.

A Figura 2.4 apresenta gráficos de discrepâncias relativas dos quantis, das diferentes estatísticas para teste das hipóteses $\mathcal{H}_{0}: \gamma_{2}=0(r=1), \mathcal{H}_{0}: \gamma_{2}=\gamma_{3}=0(r=2)$ e $\mathcal{H}_{0}: \gamma_{2}=\gamma_{3}=\gamma_{4}=0(r=3)$. Da mesma forma que no 'modelo 1', os quantis assintóticos são obtidos de uma distribuição $\chi_{1}^{2}, \chi_{2}^{2}$ e $\chi_{3}^{2}$, respectivamente. Nota-se que no 'modelo 2.1' a distribuição $\chi_{r}^{2}$ não é uma aproximação satisfatória para a distribuição nula de $w$ para diferentes tamanhos de amostra $(n)$. De fato, o teste que usa $w$ é mais liberal do que o teste ajustado em todas as situações apresentadas. A distribuição de referência está razoavelmente próxima da distribuição da estatística ajustada $w^{*}$. Os melhores desempenhos são alcançados pelos testes que se baseiam nas estatísticas $w^{*}$ e escore, com relativa vantagem para o teste escore. Nota-se que à medida que o número de observações cresce há uma melhora significativa em relação à proximidade entre a distribuição de referência e as distribuições das estatísticas, isto é, as distorções de tamanho dos testes são reduzidas, em especial no que se refere à estatística ajustada $w^{*}$. Para $n=70$ a distribuição da estatística ajustada é praticamente igual às das distribuições de referência $\chi_{1}^{2}$ e $\chi_{2}^{2}$. A distribuição da estatística escore está próxima da distribuição de referência, mas o teste que usa $S_{R}$ é conservador, com tamanhos menores que os níveis nominais.

A Tabela 2.6 apresenta taxas de rejeição não nula (poder) dos testes das três hipóteses nulas para o modelo 2.1 com $n=50$ e $\alpha=10 \%$. Aqui, as taxas de rejeição foram obtidas sob a hipótese alternativa $\gamma_{2}=\epsilon(r=1), \gamma_{2}=\gamma_{3}=\epsilon(r=2)$ e $\gamma_{2}=\gamma_{3}=\gamma_{4}=\epsilon(r=3)$ para diferentes valores de $\epsilon$. Os poderes dos testes são similares, com pequena vantagem para o teste ajustado para valores positivos de $\epsilon$. As conclusões obtidas para o 'modelo 2.1', a partir da Tabela 2.6, não se alteram em relação às conclusões para os modelos ' 1 ' e '2'. 

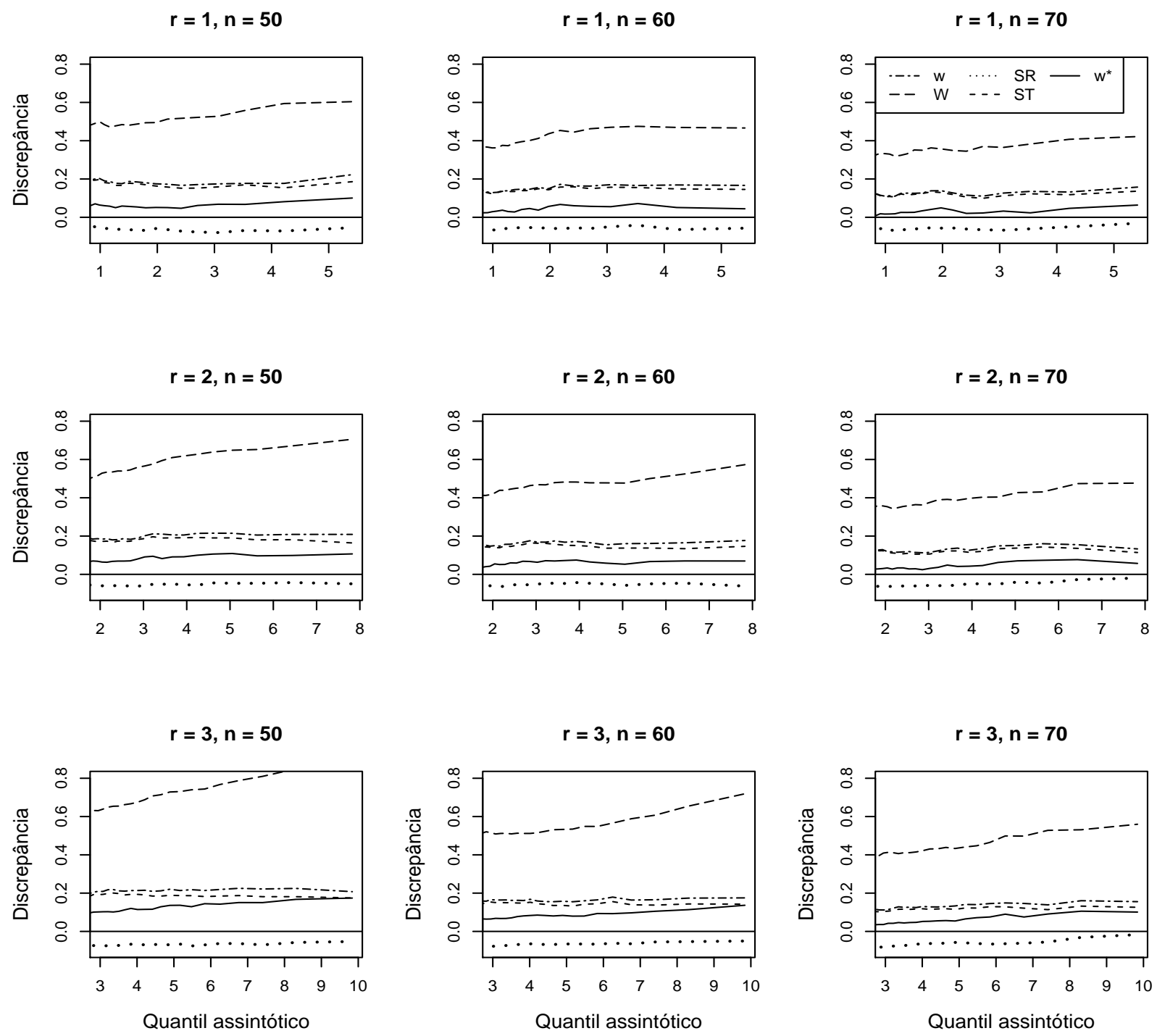

Figura 2.4: Discrepâncias relativas de quantis; modelo 2.1, dispersão.

\subsubsection{Modelo de regressão valor extremo máximo não linear com dispersão constante}

Considera-se o modelo (2.1) com parâmetro de dispersão constante e com componente sistemático da localização dado por

$$
\mu_{t}=\beta_{0}+\beta_{1} x_{t 1}+x_{t 2}^{\beta_{2}}
$$

o qual denominamos de 'modelo 3'.

Neste caso particular, temos que $X$ é uma matriz $n \times 3$, cuja $t$-ésima linha é $\left(1, x_{t 1}, \ln \left(x_{t 2}\right) x_{t 2}^{\beta_{2}}\right), Z=\iota$, $T=\mathcal{I}, H=\mathcal{I}, S$ e $Q$ são matrizes nulas, $\dot{X}=\partial^{2} \eta / \partial \beta \partial \beta^{\top}$ é tal que $\partial^{2} \eta_{t} / \partial \beta_{2} \partial \beta_{2}=\ln \left(x_{t 2}\right)^{2} x_{t 2}^{\beta_{2}}$ para $t=1, \ldots, n$ e zero caso contrário, $\dot{Z}=\partial^{2} \delta / \partial \gamma \partial \gamma^{\top}$ é o array nulo $n \times m \times m$. O produto 'colchete' da matriz $\left[\iota^{\top}\left(\mathcal{I}-\mathcal{Z}^{\dagger}\right) T \Phi^{-1}\right]$ de dimensão $1 \times n$, pelo array $\dot{X}$ de dimensão $n \times 3 \times 3$, é um array $1 \times 3 \times 3$, 
Tabela 2.6: Taxas de rejeição não nula (\%); modelo 2.1 , dispersão, $n=50, \alpha=10 \%$.

\begin{tabular}{c|c|cccccccccc}
\hline \hline $\mathrm{r}$ & $\epsilon$ & $-2,0$ & $-1,5$ & $-1,0$ & $-0,5$ & $-0,3$ & 0,3 & 0,5 & 1,0 & 1,5 & 2,0 \\
\hline \multirow{4}{*}{1} & $w$ & 98,4 & 90,6 & 62,9 & 25,3 & 15,3 & 17,7 & 29,1 & 67,3 & 92,0 & 99,0 \\
& $W$ & 98,7 & 90,6 & 61,2 & 23,7 & 14,5 & 17,6 & 28,8 & 66,9 & 92,5 & 99,2 \\
& $S_{R}$ & 97,8 & 89,5 & 63,4 & 26,7 & 16,0 & 16,9 & 27,6 & 64,4 & 89,9 & 98,3 \\
& $S_{T}$ & 98,5 & 90,8 & 63,3 & 25,7 & 15,7 & 17,5 & 28,8 & 66,9 & 92,0 & 99,0 \\
& $w^{*}$ & 98,8 & 91,8 & 65,4 & 27,9 & 16,9 & 16,0 & 26,8 & 64,5 & 90,9 & 98,8 \\
\hline \multirow{6}{*}{2} & $w$ & 100,0 & 99,6 & 91,3 & 41,8 & 21,5 & 23,7 & 45,8 & 93,0 & 99,9 & 100,0 \\
& $W$ & 100,0 & 99,8 & 90,7 & 39,3 & 20,7 & 21,7 & 42,8 & 92,1 & 99,9 & 100,0 \\
& $S_{R}$ & 100,0 & 99,3 & 89,2 & 41,6 & 21,8 & 23,6 & 44,8 & 91,3 & 99,7 & 100,0 \\
& $S_{T}$ & 100,0 & 99,7 & 91,4 & 42,1 & 21,6 & 23,6 & 45,5 & 92,9 & 99,9 & 100,0 \\
& $w^{*}$ & 100,0 & 99,7 & 91,7 & 43,3 & 22,3 & 22,4 & 43,7 & 92,0 & 99,9 & 100,0 \\
\hline \multirow{6}{*}{3} & $w$ & 100,0 & 99,9 & 94,4 & 45,1 & 22,6 & 23,5 & 47,7 & 95,4 & 99,9 & 100,0 \\
& $W$ & 100,0 & 99,9 & 93,3 & 39,6 & 20,0 & 20,7 & 40,9 & 93,8 & 99,9 & 100,0 \\
& $S_{R}$ & 100,0 & 99,7 & 92,6 & 46,1 & 23,8 & 24,2 & 47,2 & 93,8 & 99,8 & 100,0 \\
& $S_{T}$ & 100,0 & 99,9 & 94,5 & 45,4 & 22,8 & 23,3 & 47,3 & 95,3 & 99,9 & 100,0 \\
& $w^{*}$ & 100,0 & 99,9 & 94,4 & 44,9 & 22,7 & 23,0 & 45,9 & 95,0 & 99,9 & 100,0 \\
\hline \hline
\end{tabular}

isto é, uma matriz $3 \times 3$, cujo elemento $(i, j)$ é

$$
\sum_{t=1}^{n}\left\{\left(\frac{1}{\sigma_{t}}-\frac{1}{\sigma_{t}} \exp \left(-\frac{y_{t}-\mu_{t}}{\sigma_{t}}\right)\right) \frac{1}{g^{\prime}\left(\mu_{t}\right)} \ln \left(x_{t 2}\right)^{2} x_{t 2}^{\beta_{2}}\right\}
$$

se $(i, j)=(3,3)$ e zero caso contrário.

Considera-se a hipótese nula $\mathcal{H}_{0}: \beta_{2}=0(r=1)$ a ser testada contra uma alternativa bilateral. Assumese $\sigma=0,1, \beta_{0}=1, \beta_{1}=1$ e $\beta_{2}=0$. Os valores das covariadas foram obtidos aleatoriamente de uma distribuição $\mathcal{U}(0,1)$. Os tamanhos das amostras utilizados são 15, 2030 e 40. Os resultados são resumidos nas Tabelas 2.7 e 2.8 e na Figura 2.5 .

Tabela 2.7: Taxas de rejeição nula (\%); modelo 3

\begin{tabular}{c|ccccc|ccccc|ccccc}
\hline \hline & \multicolumn{7}{|c|}{$\alpha=10 \%$} & \multicolumn{5}{c|}{$\alpha=5 \%$} & \multicolumn{5}{c}{$\alpha=1 \%$} \\
\cline { 2 - 16 }$n$ & $w$ & $W$ & $S_{R}$ & $S_{T}$ & $w^{*}$ & $w$ & $W$ & $S_{R}$ & $S_{T}$ & $w^{*}$ & $w$ & $W$ & $S_{R}$ & $S_{T}$ & $w^{*}$ \\
\hline 15 & 16,6 & 22,2 & 10,2 & 13,4 & 10,0 & 9,8 & 15,8 & 4,6 & 6,3 & 5,0 & 2,9 & 8,2 & 0,9 & 1,1 & 1,1 \\
20 & 14,1 & 19,2 & 9,5 & 11,9 & 9,9 & 7,7 & 12,7 & 4,5 & 5,7 & 4,8 & 2,2 & 5,8 & 1,1 & 1,0 & 1,1 \\
30 & 12,5 & 16,3 & 9,3 & 11,1 & 9,7 & 6,8 & 10,3 & 4,6 & 5,7 & 5,1 & 1,5 & 3,9 & 1,3 & 1,0 & 0,9 \\
40 & 12,2 & 15,1 & 9,8 & 11,2 & 10,3 & 6,7 & 9,3 & 4,9 & 5,6 & 5,2 & 1,5 & 3,4 & 1,4 & 1,1 & 1,0 \\
\hline \hline
\end{tabular}

A Tabela 2.7 mostra que no 'modelo 3' o comportamento dos testes é similar ao comportamento para o caso linear homoscedástico. Os testes que usam $w$ e $W$ são tipicamente liberais enquanto a taxa de rejeição dos outros testes estão bem mais próximas dos níveis nominais com destaque para para os testes que usam a estatística escore e a ajustada. Por exemplo, para $n=15$ e $\alpha=10 \%$, a taxa de rejeição nula do teste que utiliza $w$ é $16,6 \%$, enquanto a do teste baseado nas estatísticas $w^{*}$ e escore são $10 \%$ e $10,2 \%$, respectivamente. 

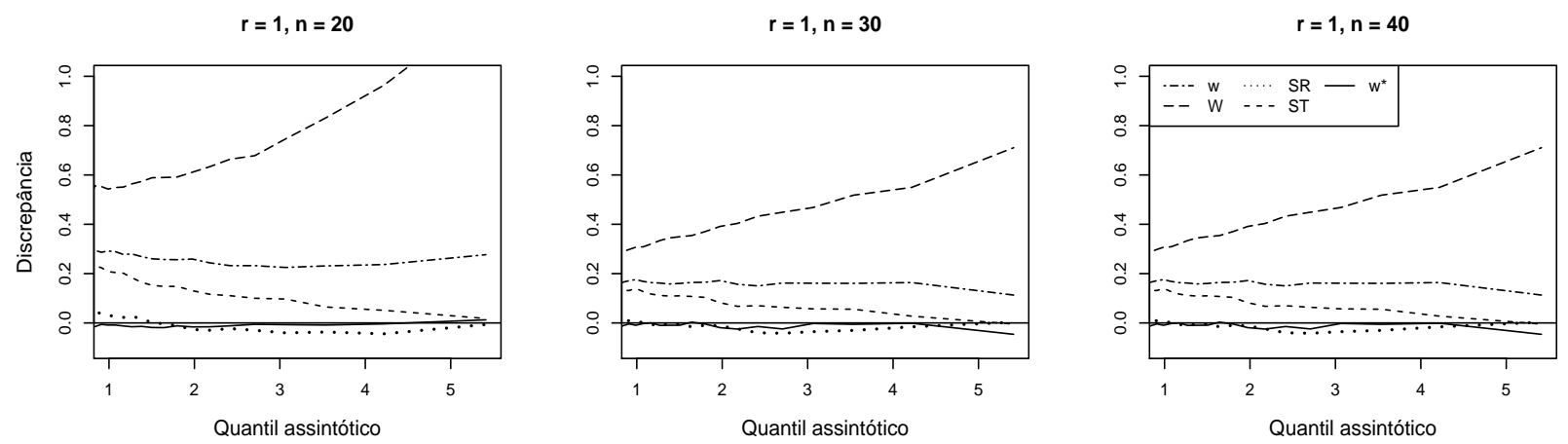

Figura 2.5: Discrepâncias relativas de quantis; modelo 3.

A Figura 2.5 mostra que a distribuição de referência $\chi_{1}^{2}$ não é uma aproximação satisfatória para a distribuição nula de $w$ e de $W$, mas está próxima das distribuições das estatísticas ajustada e escore e, ainda, para $n=20$ são praticamente iguais. A distribuição da estatística gradiente está mais da próxima da distribuição de referência comparando-se com a estatística $w$, e está bem próxima para quantis grandes.

Tabela 2.8: Taxas de rejeição não nula (\%); modelo $3, n=15, \alpha=10 \%$.

\begin{tabular}{c|cccccccccc}
\hline \hline$\epsilon$ & $-0,3$ & $-0,2$ & $-0,1$ & $-0,05$ & $-0,03$ & 0,03 & 0,05 & 0,1 & 0,2 & 0,3 \\
\hline$w$ & 100,0 & 100,0 & 80,4 & 32,8 & 18,3 & 18,0 & 29,6 & 65,8 & 96,0 & 99,4 \\
$W$ & 100,0 & 100,0 & 86,6 & 39,1 & 22,0 & 11,2 & 17,8 & 48,4 & 91,3 & 99,0 \\
$S_{R}$ & 99,9 & 97,0 & 59,3 & 21,3 & 11,7 & 23,3 & 37,7 & 72,4 & 95,8 & 99,1 \\
$S_{T}$ & 100,0 & 99,9 & 74,5 & 27,0 & 15,1 & 21,2 & 34,3 & 71,2 & 97,1 & 99,7 \\
$w^{*}$ & 100,0 & 99,9 & 75,5 & 27,5 & 15,3 & 20,8 & 33,6 & 70,3 & 97,1 & 99,7 \\
\hline \hline
\end{tabular}

A Tabela 2.8 apresenta taxas de rejeição não nula (poder) dos testes para o modelo 3 com $n=15$ e $\alpha=10 \%$. Aqui, as taxas de rejeição foram obtidas sob a hipótese alternativa $\beta_{2}=\epsilon(r=1)$, para diferentes valores de $\epsilon$. Os resultados obtidos para o 'modelo 3', mostrados na Tabela 2.8, revelam a semelhança dos testes em termos de poder, em particular a proximidade entre os poderes dos testes gradiente e ajustado. As conclusões obtidas para o 'modelo 3' não se alteram em relação às conclusões para os modelos ' 1 ' e '2'.

\subsubsection{Modelo de regressão valor extremo máximo não linear com dispersão variável}

Considera-se o modelo (2.1) com os componentes sistemáticos não linear para a localização e linear para dispersão dados por

$$
\mu_{t}=\beta_{0}+\mathrm{e}^{\left(\beta_{1}+\beta_{2} x_{t}\right)}
$$

e

$$
\ln \left(\sigma_{t}\right)=\gamma_{0}+\gamma_{1} z_{t}
$$

o qual denominamos de 'modelo 4'.

Neste caso particular, temos que $X$ é uma matriz $n \times 3$, cuja $t$-ésima linha é $\left(1, \mathrm{e}^{\beta_{1}+\beta_{2} x_{t}}, x_{t} \mathrm{e}^{\beta_{1}+\beta_{2} x_{t}}\right), Z$ é uma matriz $n \times 2$, cuja $t$-ésima linha é $\left(1, z_{t}\right), \Phi=\operatorname{diag}\left\{\sigma_{1} \ldots, \sigma_{n}\right\}, T=\mathcal{I}, H=\operatorname{diag}\left\{\sigma_{1} \ldots, \sigma_{n}\right\}, S$ é a 
matriz nula, $Q=\operatorname{diag}\left\{-1 / \sigma_{1}^{2}, \ldots,-1 / \sigma_{n}^{2}\right\}, \dot{X}=\partial^{2} \eta / \partial \beta \partial \beta^{\top}$ é tal que $\partial^{2} \eta_{t} / \partial \beta_{1} \partial \beta_{1}=\exp \left(\beta_{1}+\beta_{2} x_{t}\right)$, $\partial^{2} \eta_{t} / \partial \beta_{1} \partial \beta_{2}=\partial^{2} \eta_{t} / \partial \beta_{2} \partial \beta_{1}=x_{t} \exp \left(\beta_{1}+\beta_{2} x_{t}\right), \partial^{2} \eta_{t} / \partial \beta_{2} \partial \beta_{2}=x_{t}^{2} \exp \left(\beta_{1}+\beta_{2} x_{t}\right), t=1, \ldots, n \mathrm{e}$ zero caso contrário, $\dot{Z}=\partial^{2} \delta / \partial \gamma \partial \gamma^{\top}$ é o array nulo $n \times 2 \times 2$. O produto 'colchete' da matriz $\left[\iota^{\top}(\mathcal{I}-\right.$ $\left.\left.\mathcal{Z}^{\dagger}\right) T \Phi^{-1}\right]$ de dimensão $1 \times n$ pelo array $\dot{X}$ de dimensão $n \times 3 \times 3$ é a matriz $3 \times 3$, cujos elementos são

$$
\begin{cases}\sum_{t=1}^{n}\left\{\left(\frac{1}{\sigma_{t}}-\frac{1}{\sigma_{t}} \exp \left(-\frac{y_{t}-\mu_{t}}{\sigma_{t}}\right)\right) \frac{1}{g^{\prime}\left(\mu_{t}\right)} \exp \left(\beta_{1}+\beta_{2} x_{t}\right)\right\}, & \text { se }(i, j)=(2,2) \\ \left.\left(\frac{1}{\sigma_{t}}-\frac{1}{\sigma_{t}} \exp \left(-\frac{y_{t}-\mu_{t}}{\sigma_{t}}\right)\right) \frac{1}{g^{\prime}\left(\mu_{t}\right)} x_{t} \exp \left(\beta_{1}+\beta_{2} x_{t}\right)\right\}, & \text { se }(i, j)=(2,3),(3,2) \\ \sum_{t=1}^{n}\left\{\left(\frac{1}{\sigma_{t}}-\frac{1}{\sigma_{t}} \exp \left(-\frac{y_{t}-\mu_{t}}{\sigma_{t}}\right)\right) \frac{1}{g^{\prime}\left(\mu_{t}\right)} x_{t}^{2} \exp \left(\beta_{1}+\beta_{2} x_{t}\right)\right\}, & \text { se }(i, j)=(3,3) \\ 0, & \text { cc. }\end{cases}
$$

Considera-se a hipótese nula $\mathcal{H}_{0}: \gamma_{1}=0(r=1)$, a ser testada contra uma alternativa bilateral. Assume-se $\gamma_{0}=1$ e $\beta_{0}=1, \beta_{1}=1$ e $\beta_{2}=6$. Os valores das covariadas foram obtidos aleatoriamente de uma distribuição $\mathcal{U}(-0,5,0,5)$. Os tamanhos das amostras utilizados são 30, 40, 50 e 60. Os resultados são resumidos nas Tabelas 2.9 e 2.10 e na Figura 2.6.

Tabela 2.9: Taxas de rejeição nula $(\%)$; modelo 4 , dispersão

\begin{tabular}{c|ccccc|cccccc|ccccc}
\hline \hline & \multicolumn{6}{|c|}{$\alpha=10 \%$} & \multicolumn{5}{c|}{$\alpha=5 \%$} & \multicolumn{5}{c}{$\alpha=1 \%$} \\
\cline { 2 - 16 }$n$ & $w$ & $W$ & $S_{R}$ & $S_{T}$ & $w^{*}$ & $w$ & $W$ & $S_{R}$ & $S_{T}$ & $w^{*}$ & $w$ & $W$ & $S_{R}$ & $S_{T}$ & $w^{*}$ \\
\hline 30 & 14,3 & 20,6 & 9,1 & 14,0 & 11,8 & 7,8 & 13,0 & 4,4 & 7,7 & 6,4 & 2,1 & 5,2 & 0,8 & 2,0 & 1,5 \\
40 & 12,5 & 16,8 & 9,0 & 12,5 & 10,7 & 6,9 & 10,3 & 4,5 & 6,7 & 5,7 & 1,7 & 3,7 & 0,8 & 1,6 & 1,3 \\
50 & 11,7 & 15,5 & 8,8 & 11,7 & 10,4 & 6,0 & 9,1 & 4,3 & 6,0 & 5,7 & 1,5 & 2,9 & 0,9 & 1,5 & 1,4 \\
60 & 12,0 & 14,5 & 10,0 & 11,9 & 10,8 & 6,4 & 8,6 & 4,9 & 6,3 & 5,6 & 1,5 & 2,7 & 0,9 & 1,5 & 1,3 \\
\hline \hline
\end{tabular}

A Tabela 2.9 mostra que no 'modelo 4' os comportamentos dos testes são similares aos modelos anteriores. Os testes escore e ajustado são os que melhor se aproximam da distribuição de referência, como é possível observar no caso de $n=40$ e $\alpha=10 \%$, em que as taxas de rejeição para os testes que usam as estatísticas $S_{R}$ e $w^{*}$ são, respectivamente, $9,0 \%$ e $10,7 \%$, enquanto os testes $w, W$ e $S_{T}$ são liberais apresentando taxas de rejeição $12,5 \%, 16,8 \%$ e $12,5 \%$, respectivamente.
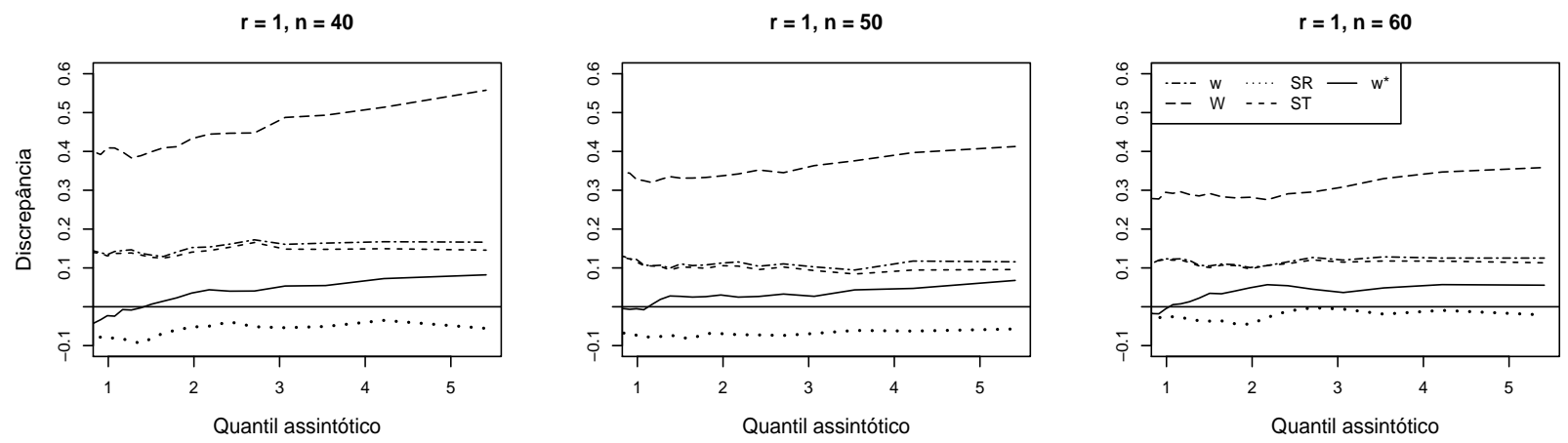

Figura 2.6: Discrepâncias relativas de quantis; modelo 4, dispersão.

A Figura 2.6 mostra que a distribuição $\chi_{1}^{2}$ não é uma aproximação satisfatória para a distribuição nula 
de $w$ e $W$. Por outro lado, a distribuição da estatística ajustada está bem mais próxima da estatística de referência do que a usual. A estatística escore é competitiva tendo sua distribuição próxima da distribuição de referência, especialmente para $n=60$, mas mantém sua característica conservadora de rejeitar a hipótese nula com frequência menor do que o nível nominal.

Tabela 2.10: Taxas de rejeição não nula (\%); modelo 4, dispersão, $n=40, \alpha=10 \%$.

\begin{tabular}{c|cccccccccc}
\hline \hline$\epsilon$ & $-1,5$ & -1 & $-0,5$ & $-0,3$ & $-0,1$ & 0,1 & 0,3 & 0,5 & 1 & 1,5 \\
\hline$w$ & 91,5 & 67,2 & 30,6 & 18,6 & 11,8 & 9,2 & 11,1 & 17,4 & 49,7 & 82,1 \\
$W$ & 91,4 & 66,4 & 29,6 & 18,3 & 11,8 & 9,4 & 10,9 & 17,3 & 48,8 & 82,5 \\
$S_{R}$ & 89,7 & 66,0 & 30,9 & 18,9 & 11,8 & 9,3 & 10,6 & 16,8 & 47,5 & 79,1 \\
$S_{T}$ & 91,5 & 67,3 & 30,7 & 18,7 & 11,8 & 9,2 & 11,1 & 17,2 & 49,4 & 82,0 \\
$w^{*}$ & 87,7 & 59,8 & 23,8 & 14,6 & 10,5 & 10,5 & 15,6 & 24,9 & 60,4 & 88,1 \\
\hline \hline
\end{tabular}

A Tabela 2.10 apresenta taxas de rejeição não nula (poder) dos testes de $\mathcal{H}_{0}: \gamma_{1}=0$ para o modelo 4 com $n=40$ e $\alpha=10 \%$. Aqui, as taxas de rejeição foram obtidas sob a hipóteses alternativa $\gamma_{1}=\epsilon(r=1)$ para diferentes valores de $\epsilon$.

Os resultados obtidos para o 'modelo 4', mostrados na Tabela 2.10, revelam que não há um teste com poder maior em todas as situações, tendo o teste ajustado poder maior para valores positivos de $\epsilon$ e menor para valores negativos de $\epsilon$. As conclusões obtidas para o 'modelo 4' não se alteram em relação às conclusões para os modelos ' 1 ', '2' e ' 3 '.

Em resumo, dentre os cinco testes analisados $w, W, S_{R}, S_{T}$ e $w^{*}$, o teste da razão de verossimilhanças ajustada $w^{*}$ é o que produz, em geral, taxas de rejeição nula mais próximas dos níveis nominais especialmente quando $n$ é pequeno. Em outras palavras, $w^{*}$ é o teste mais confiável no que diz respeito à probabilidade de erro de tipo I. Adicionalmente, os testes são igualmente poderosos quando corrigidos para terem probabilidade de erro de tipo I iguais. Embora a estatística da razão de verossimilhanças ajustada seja mais difícil de se obter o teste que usa $w^{*}$ é menos liberal na maioria dos casos e, por isso, recomendado para aplicações práticas. O teste que usa a estatística escore é conservador com as taxas de rejeição próximas do nível nominal, sendo especialmente competitivo nos testes relativos ao parâmetro de dispersão, portanto, recomendado nestes testes para aplicações práticas.

\subsection{Aplicações}

Nesta seção aplicaremos os resultados desenvolvidos até aqui a três conjuntos reais de dados. O primeiro é apresentado na Tabela 2.11. Este conjunto de dados, aqui denominado trigo, foi analisado anteriormente em Huet et al. (2004), Faivre \& Masle (1988) e Barreto-Souza \& Vasconcellos (2011, p. 8). Considera-se o crescimento do trigo durante o inverno observando a diferença de peso das hastes quando já estão secas. A variável explicativa $(x)$ é medida numa escala de graus-dias cumulativa. Esta medida é uma integral no tempo de todas as temperaturas a que o trigo é submetido, que são maiores que a temperatura mínima na qual o trigo se desenvolve. A temperatura é medida em graus Celsius e o tempo é medido em dias, sendo que o tempo de início é definido pelo estado fisiológico do trigo. Plantas são colhidas a cada semana em $n=18$ áreas de aproximadamente $0,15 \mathrm{~m}^{2}$, escolhidas aleatoriamente. As plantas são pesadas depois de 
Tabela 2.11: Peso de hastes de trigo seco.

\begin{tabular}{c|cccc}
\hline \hline & \multicolumn{4}{|c}{$\operatorname{Peso}(\mathrm{mg})(y)$} \\
Graus-dias $(x)$ & \multicolumn{5}{|c}{} \\
\hline 405,65 & 113,386 & 90,500 & & \\
498,75 & 161,600 & 207,650 & & \\
567,25 & 309,514 & 246,743 & & \\
618,30 & 460,686 & 422,936 & & 1034,000 \\
681,45 & 1047,000 & 972,383 & 1072,022 & 1266,290 \\
681,45 & 1169,767 & 1141,883 & 999,633 & 120 \\
681,45 & 868,662 & 1133,287 & & \\
\hline \hline
\end{tabular}

secas e os valores são fornecidos em miligramas. Para uma descrição detalhada dos dados ver Huet et al. (2004, p. 61).

Barreto-Souza \& Vasconcellos (2011) assumem que o peso da haste quando está seca (y) segue uma distribuição valor extremo máximo (2.1) e consideram um modelo de regressão não linear. O modelo tem o componente sistemático da locação dado por

$$
\mu_{t}=\beta_{0}+\mathrm{e}^{\beta_{1}+\beta_{2} x_{t}}
$$

e especificação da dispersão dada por

$$
\ln \sigma_{t}=\gamma_{1} x_{t}
$$

para $t=1, \ldots, 18$, o qual chamamos de 'modelo I'.

As estimativas de máxima verossimilhança dos parâmetros para o 'modelo I' são, como esperado, as mesmas de Barreto-Souza \& Vasconcellos (2011, p. 9) e são apresentadas na Tabela 2.12 .

Tabela 2.12: Estimativas dos parâmetros e erros padrão: modelo I - trigo

\begin{tabular}{lcccc} 
Parâmetros & $\beta_{0}$ & $\beta_{1}$ & $\beta_{2}$ & $\gamma_{1}$ \\
\hline & & & & \\
Descrição & Intercepto & - & Graus-dias & Graus-dias \\
\hline EMV & 81,6 & $-2,741$ & 0,01404 & 0,00661 \\
EP & 14,7 & 0,928 & 0,00135 & 0,00030 \\
\hline \hline
\end{tabular}

Os intervalos de confiança com coeficiente de confiança de $95 \%$ obtidos pela inversão dos testes da razão de verossimilhanças, Wald, escore, gradiente e razão de verossimilhanças ajustada são apresentados na Tabela 2.13 e na Figura 2.7. Os comprimentos dos intervalos obtidos dos testes da razão de verossimilhanças e de Wald são menores do que os dos intervalos baseados nos outros testes, como é de se esperar já que são os testes mais liberais.

Barreto-Souza \& Vasconcellos (2011, p. 9) obtêm os seguintes intervalos de confiança com base em estimadores de máxima verossimilhança corrigidos por viés: $I C\left(\beta_{0}, 95 \%\right)=(48,4 ; 111,0), I C\left(\beta_{1}, 95 \%\right)=$ 
Tabela 2.13: Intervalos de confiança com coeficiente de confiança de 95\% - trigo

\begin{tabular}{cccccc}
\hline \hline & & & & \\
& $w$ & $W$ & $S_{R}$ & $S_{T}$ & $w^{*}$ \\
\hline$\beta_{0}$ & $(47,6 ; 104,7)$ & $(52,7 ; 110,5)$ & $(31,4 ; 100,5)$ & $(38,6 ; 103,7)$ & $(39,7 ; 104,9)$ \\
$\beta_{1}$ & $(-5,08 ;-1,11)$ & $(-4,56 ;-0,92)$ & $(-7,60 ;-1,20)$ & $(-6,04 ;-1,11)$ & $(-5,49 ;-0,96)$ \\
$\beta_{2}$ & $(0,0117 ; 0,0175)$ & $(0,0114 ; 0,0167)$ & $(0,0118 ; 0,0213)$ & $(0,0117 ; 0,0189)$ & $(0,0115 ; 0,0181)$ \\
$\gamma_{1}$ & $(0,00610 ; 0,00723)$ & $(0,00603 ; 0,00720)$ & $(0,00619 ; 0,00740)$ & $(0,00612 ; 0,00727)$ & $(0,00622 ; 0,00751)$ \\
\hline \hline
\end{tabular}
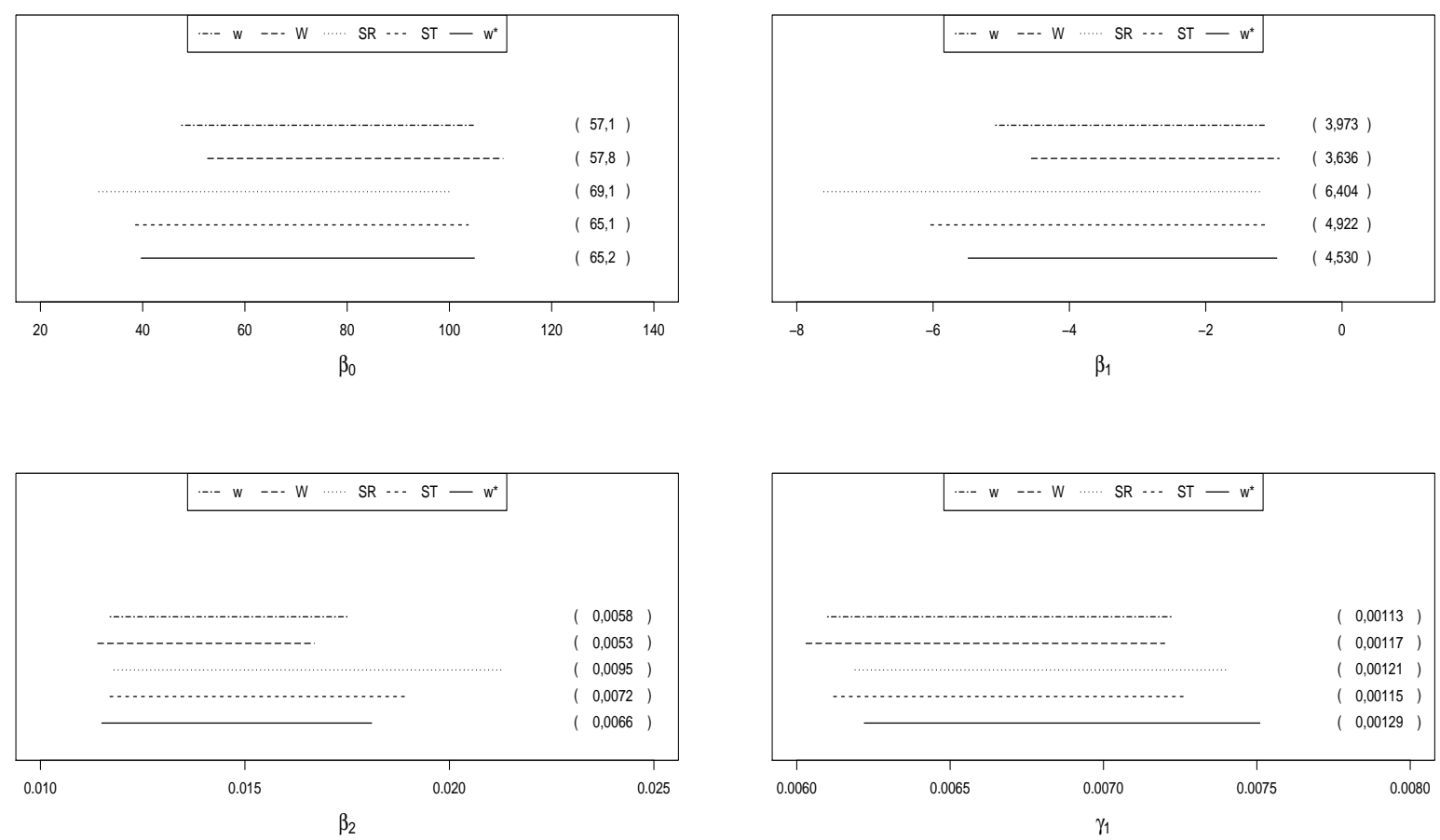

Figura 2.7: Intervalos de confiança com coeficiente de confiança de 95\%; comprimentos dos intervalos entre parênteses - trigo.

$(-4,71 ;-0,67), I C\left(\beta_{2}, 95 \%\right)=(0,0110 ; 0,0169)$ e $I C\left(\gamma_{0}, 95 \%\right)=(0,00622 ; 0,00738)$. A correção de viés do estimador de máxima verossimilhança elimina o viés, isto é, garante que a esperança do estimador corrigido é o parâmetro estimado a menos de um erro de ordem $O\left(n^{-2}\right)$ mas não garante que o estimador corrigido tenha distribuição mais próxima da distribuição normal.

Os resultados dos testes da hipótese nula $\mathcal{H}_{0}: \beta_{2}=\beta_{c}$ contra $\mathcal{H}_{1}: \beta_{2} \neq \beta_{c}$ obtidos para diferentes valores de $\beta_{c}$ para este modelo são apresentados na Tabela 2.14. Podemos observar que a inferência baseada nos cinco testes, ao nível de significância de $1 \%$, não rejeita a hipótese nula $\mathcal{H}_{0}: \beta_{2}=0,011$, que é o limite inferior do intervalo de confiança com $95 \%$ de confiança baseado no estimador corrigido de Barreto-Souza \& Vasconcelos (2011, p. 9). Ao nível de significância de 10\%, o teste baseado na estatística corrigida ( $w^{*}$ ) não rejeita a hipótese nula $\mathcal{H}_{0}: \beta_{2}=0,017$, que não pertence ao intervalo de confiança com $95 \%$ de confiança baseado no estimador de máxima verossimilhança corrigido de Barreto-Souza \& Vasconcellos (2011, p. 9). No entanto, é rejeitada pelo teste baseado na estatística da razão de verossimilhanças assim como pelo teste 


\begin{tabular}{|c|c|c|c|c|c|c|}
\hline$\beta_{c}$ & & $w$ & $W$ & $S_{R}$ & $S_{T}$ & $w^{*}$ \\
\hline \multirow[t]{2}{*}{0,0100} & estatística & 10,3119 & 8,9153 & 8,8549 & 8,7718 & 8,3784 \\
\hline & valor-p & 0,0013 & 0,0028 & 0,0029 & 0,0031 & 0,0038 \\
\hline \multirow[t]{2}{*}{0,0110} & estatística & 6,1776 & 5,0506 & 6,2264 & 5,8526 & 5,1078 \\
\hline & valor-p & 0,0129 & 0,0246 & 0,0126 & 0,0156 & 0,0238 \\
\hline \multirow[t]{2}{*}{0,0120} & estatística & 2.8032 & 2.2767 & 3.1438 & 2.8718 & 2.4440 \\
\hline & valor-p & 0.0941 & 0.1313 & 0.0762 & 0.0901 & 0.1180 \\
\hline \multirow[t]{2}{*}{0,0125} & estatística & 1.5671 & 1.2987 & 1.7906 & 1.6358 & 1.4542 \\
\hline & valor-p & 0.2106 & 0.2544 & 0.1809 & 0.2009 & 0.2279 \\
\hline \multirow[t]{2}{*}{0,0150} & estatística & 0.4469 & 0.4993 & 0.4024 & 0.4129 & 0.3958 \\
\hline & valor-p & 0.5038 & 0.4798 & 0.5259 & 0.5205 & 0.5292 \\
\hline \multirow[t]{2}{*}{0,0170} & estatística & 3,0709 & 4,7682 & 2,0317 & 2,3246 & 2,4406 \\
\hline & valor-p & 0,0797 & 0,0290 & 0,1540 & 0,1273 & 0,1182 \\
\hline \multirow[t]{2}{*}{0,0175} & estatística & 3.8683 & 6.5171 & 2.3838 & 2.7855 & 3.0820 \\
\hline & valor-p & 0.0492 & 0.0107 & 0.1226 & 0.0951 & 0.0792 \\
\hline \multirow[t]{2}{*}{0,0180} & estatística & 4,6775 & 8,5387 & 2,6938 & 3,2065 & 3,7374 \\
\hline & valor-p & 0,0306 & 0,0035 & 0,1007 & 0,0733 & 0,0532 \\
\hline \multirow[t]{2}{*}{0,0190} & estatística & 6,2830 & 13,4000 & 3,1929 & 3,9155 & 5,0476 \\
\hline & valor-p & 0,0122 & 0,0003 & 0,0740 & 0,0478 & 0,0247 \\
\hline \multirow[t]{2}{*}{0,0200} & estatística & 7,8223 & 19,3520 & 3,5517 & 4,4497 & 6,3126 \\
\hline & valor-p & 0,0052 & 0,0000 & 0,0595 & 0,0349 & 0,0120 \\
\hline
\end{tabular}

baseado na estatística de Wald. Ao nível de significância de 5\%, o teste baseado na estatística corrigida $\left(w^{*}\right)$ não rejeita a hipótese nula $\mathcal{H}_{0}: \beta_{2}=0,018$, no entanto, é rejeitada pelo teste baseado na estatística da razão de verossimilhanças assim como pelo teste baseado na estatística de Wald. E ainda, ao nível de significância de $1 \%$, o teste baseado na estatística corrigida não rejeita a hipótese nula $\mathcal{H}_{0}: \beta_{2}=0,02$, que é rejeitada pelo teste baseado na estatística da razão de verossimilhanças assim como pelo teste baseado na estatística de Wald.

O segundo conjunto de dados, chamado aqui de fremantle (Coles, 2001, p. 107), tem 86 linhas e três colunas (ver Tabela 2.15). A segunda coluna contém 86 níveis máximos anuais do mar em Fremantle, Austrália Ocidental, no período 1897-1989. A primeira coluna indica o ano correspondente. A terceira coluna dá valores médios anuais do Índice de Oscilação Sul (SOI) que indica a volatilidade meteorológica. Assumimos que o nível do mar segue uma distribuição valor extremo máximo (2.1) e consideramos alguns modelos de regressão. O primeiro modelo considerado tem componente sistemático da locação dado por

$$
\mu_{t}=\beta_{0}+\beta_{1} x_{t 1}+\beta_{2} x_{t 2}+\beta_{3} x_{t 3}
$$

e especificação da dispersão dada por

$$
\ln \sigma_{t}=\gamma_{0}+\gamma_{1} x_{t 1}+\gamma_{2} x_{t 2}+\gamma_{3} x_{t 3}
$$


para $t=1, \ldots, 86$, o qual chamamos de 'modelo I'. As covariadas são $x_{1}=S O I, x_{2}=$ tempo, $x_{3}=$ tempo $\times S O I$. Os resultados dos testes da hipótese nula $\mathcal{H}_{0}: \gamma_{3}=0$ contra $\mathcal{H}_{1}: \gamma_{3} \neq 0$ são apresentados na Tabela 2.16. Como nenhuma das estatísticas rejeita a hipótese nula, consideramos o modelo com mesmo componente sistemático para a locação e especificação da dispersão dada por

$$
\ln \sigma_{t}=\gamma_{0}+\gamma_{1} x_{t 1}+\gamma_{2} x_{t 2}
$$

para $t=1, \ldots, 86$, o qual chamamos de 'modelo II'. Os resultados dos testes da hipótese nula $\mathcal{H}_{0}: \gamma_{2}=0$ contra $\mathcal{H}_{1}: \gamma_{2} \neq 0$ são apresentados na Tabela 2.17. Como nenhuma das estatísticas rejeita a hipótese nula ao nível de significância de $10 \%$, consideramos o modelo com mesmo componente sistemático para a locação e especificação da dispersão dada por

$$
\ln \sigma_{t}=\gamma_{0}+\gamma_{1} x_{t 1}
$$

para $t=1, \ldots, 86$, o qual chamamos de 'modelo III'. Os resultados dos testes da hipótese nula $\mathcal{H}_{0}: \beta_{3}=0$ contra $\mathcal{H}_{1}: \beta_{3} \neq 0$ são apresentados na Tabela 2.18. Como nenhuma das estatísticas rejeita a hipótese nula , consideramos o modelo com componente sistemático da locação dado por

$$
\mu_{t}=\beta_{0}+\beta_{1} x_{t 1}+\beta_{2} x_{t 2}
$$

e especificação da dispersão dada por

$$
\ln \sigma_{t}=\gamma_{0}+\gamma_{1} x_{t 1}
$$

para $t=1, \ldots, 86$, o qual chamamos de 'modelo IV'. Os resultados dos testes da hipótese nula $\mathcal{H}_{0}: \gamma_{1}=0$ contra $\mathcal{H}_{1}: \gamma_{1} \neq 0$ são apresentados na Tabela 2.19. Ao nível de significância de $10 \%$ apenas o teste escore $\left(S_{R}\right)$ não rejeita a hipótese nula. Aqui, $n$ é grande e, como esperado, não há diferenças significativas entre os valores das estatísticas de teste e entre as decisões baseadas nos testes.

O terceiro conjunto de dados, chamado aqui de "decatlo" (Hand, 1996, p. 304), tem 34 linhas e 11 colunas (ver http: / / www.stat.ncsu.edu/working_groups/sas/sicl/data/ conjunto de dados 357). As colunas contêm as distâncias e tempos de 34 atletas, na Olimpíada de 1988, nas modalidades esportivas do decatlo e, na última coluna, a pontuação final. Decatlo é uma competição de atletismo composta por dez provas. Nos Jogos Olímpicos, é exclusivamente praticada por homens. O equivalente feminino desta prova é o heptatlo, com sete provas. Os atletas inscritos competem num programa de dois dias, que inclui as seguintes modalidades: $1^{\circ}$ dia: 100 metros rasos, salto em distância, arremesso de peso, salto em altura, 400 metros rasos e $2^{\circ}$ dia: 110 metros barreiras, lançamento de disco, salto com vara, lançamento de dardo, 1500 metros. As modalidades podem ser divididas em trajeto e campo. As atividades de trajeto são medidas em unidade de tempo e o atleta almeja o tempo mínimo (100m, 400m, corrida com barreiras, e 1500m). As atividades de campo são medidas em unidade de distância e o atleta deve atingir a máxima distância (salto em distância, arremesso de peso, salto em altura, lançamento de disco, salto com vara e lançamento de dardo).

Assumimos que o resultado em salto em altura segue uma distribuição valor extremo máximo (2.1) e 
consideramos o modelo de regressão com dispersão constante e componente sistemático da locação dado por

$$
\mu_{t}=\beta_{0}+\beta_{1} x_{t 1}+\beta_{2} x_{t 2}+\beta_{3} x_{t 3}+\beta_{4} x_{t 4}+\beta_{5} x_{t 5}
$$

para $t=1, \ldots, 34$. As covariadas são os resultados nas seguintes provas: lançamento de dardo $\left(x_{1}\right)$, salto em distância $\left(x_{2}\right)$, lançamento de disco $\left(x_{3}\right)$, arremesso de peso $\left(x_{4}\right)$ e salto com vara $\left(x_{5}\right)$. Os resultados dos testes da hipótese nula $\mathcal{H}_{0}: \beta_{1}=0$ contra $\mathcal{H}_{1}: \beta_{1} \neq 0$ são apresentados na Tabela 2.21. Ao nível de significância de 5\%, apenas o teste da razão de verossimilhanças $(w)$ e Wald $(W)$ rejeitam a hipótese nula e o teste gradiente $\left(S_{T}\right)$ tem valor-p próximo do nível nominal; Ao nível de significância de $10 \%$ apenas o teste da razão de verossimilhanças corrigido $\left(w^{*}\right)$ não rejeita a hipótese nula e o teste escore $\left(S_{R}\right)$ tem valor-p próximo no nível nominal.

\subsection{Conclusões}

A regressão valor extremo pode ser uma opção apropriada para modelar eventos extremos como inundação, velocidade de vento, altura de onda ou precipitação considerando variáveis explicativas. A inferência nestes modelos baseia-se usualmente em teoria assintótica, que é frequentemente enganosa quando a amostra é pequena. É útil, portanto, deduzir ferramentas de inferência em regressão valor extremo que sejam confiáveis mesmo em amostras pequenas ou de tamanho moderado. Neste trabalho foi seguida a proposta de Skovgaard (2001) para obter um ajuste para a estatística da razão de verossimilhanças que possibilite uma boa aproximação entre a distribuição da estatística de teste e a distribuição assintótica quando a amostra não é grande. O ajuste para a estatística da razão de verossimilhanças foi obtido em modelos de regressão valor extremo lineares e não lineares com precisão variável. Compara-se a acurácia do teste da razão de verossimilhanças usual, teste de Wald, teste escore, teste gradiente e do teste da razão de verossimilhanças ajustada aqui obtido. Os resultados das simulações sugerem que o teste da razão de verossimilhanças e o teste de Wald podem ter o tamanho marcadamente superior ao nível nominal em amostras de tamanho pequeno ou moderado. O teste gradiente pode apresentar o tamanho do teste maior que o nível nominal mas bem menos do que os testes já mencionados. O teste de escore é ainda menos distorcido e pode ser conservativo em alguns casos. O teste da razão de verossimilhanças ajustado obtido neste trabalho tem um desempenho melhor que todos os outros. Embora requeira algum esforço computacional, é menos distorcido na maioria dos casos examinados e, portanto, recomendado em aplicações práticas. Deve-se enfatizar que nossas simulações foram feitas para modelos de regressão valor extremo com preditores lineares e não lineares para ambos os parâmetros de localização e escala. Todas as simulações exibiram comportamento similar.

A aplicação dos testes mencionados a conjuntos de dados reais aponta para a possibilidade de conclusão errada com base nos testes usuais quando a amostra é pequena. Assim, sugerimos nestes casos a utilização dos testes ajustados para fazer inferência. 
Tabela 2.15: Nível do mar em Fremantle, Austrália.

\begin{tabular}{|c|c|c|c|c|c|}
\hline Year $(x)$ & SeaLevel $(y)$ & SOI $(x)$ & Year $(x)$ & SeaLevel $(y)$ & SOI $(x)$ \\
\hline 1897 & 1,58 & $-0,67$ & 1947 & 1,46 & 0,16 \\
\hline 1898 & 1,71 & 0,57 & 1948 & 1,52 & $-0,24$ \\
\hline 1899 & 1,40 & 0,16 & 1949 & 1,58 & $-0,21$ \\
\hline 1900 & 1,34 & $-0,65$ & 1950 & 1,65 & 1,49 \\
\hline 1901 & 1,43 & 0,06 & 1951 & 1,49 & $-0,69$ \\
\hline 1903 & 1,19 & 0,47 & 1952 & 1,52 & $-0,23$ \\
\hline 1904 & 1,55 & 0,39 & 1953 & 1,52 & $-0,76$ \\
\hline 1905 & 1,34 & $-1,78$ & 1954 & 1,49 & 0,23 \\
\hline 1906 & 1,37 & 0,20 & 1955 & 1,62 & 0,89 \\
\hline 1908 & 1,46 & 0,28 & 1956 & 1,86 & 1,00 \\
\hline 1909 & 1,92 & 0,28 & 1957 & 1,58 & $-0,45$ \\
\hline 1912 & 1,37 & $-0,97$ & 1958 & 1,62 & $-0,50$ \\
\hline 1914 & 1,19 & $-0,92$ & 1959 & 1,46 & $-0,11$ \\
\hline 1915 & 1,40 & 0,16 & 1960 & 1,43 & 0,28 \\
\hline 1916 & 1,28 & 0,62 & 1961 & 1,46 & $-0,01$ \\
\hline 1917 & 1,52 & 2,12 & 1962 & 1,62 & 0,38 \\
\hline 1918 & 1,52 & 0,05 & 1963 & 1,68 & $-0,32$ \\
\hline 1919 & 1,58 & $-1,09$ & 1964 & 1,83 & 0,53 \\
\hline 1920 & 1,49 & 0,08 & 1965 & 1,62 & $-0,97$ \\
\hline 1921 & 1,65 & 0,66 & 1966 & 1,46 & $-0,53$ \\
\hline 1922 & 1,37 & 0,33 & 1967 & 1,58 & 0,25 \\
\hline 1923 & 1,49 & $-0,36$ & 1968 & 1,77 & 0,19 \\
\hline 1924 & 1,46 & 0,33 & 1969 & 1,62 & $-0,66$ \\
\hline 1925 & 1,34 & $-0,24$ & 1970 & 1,71 & 0,28 \\
\hline 1927 & 1,74 & 0,27 & 1971 & 1,46 & 1,06 \\
\hline 1928 & 1,62 & 0,43 & 1972 & 1,60 & $-0,88$ \\
\hline 1929 & 1,46 & 0,46 & 1973 & 1,50 & 0,63 \\
\hline 1930 & 1,71 & 0,03 & 1974 & 1,60 & 0,97 \\
\hline 1931 & 1,74 & 0,39 & 1975 & 1,90 & 1,32 \\
\hline 1932 & 1,55 & $-0,68$ & 1976 & 1,70 & 0,06 \\
\hline 1933 & 1,43 & 0,09 & 1977 & 1,40 & $-1,13$ \\
\hline 1934 & 1,62 & $-0,01$ & 1978 & 1,80 & $-0,30$ \\
\hline 1935 & 1,49 & 0,14 & 1979 & 1,37 & $-0,08$ \\
\hline 1936 & 1,58 & 0,03 & 1980 & 1,46 & $-0,43$ \\
\hline 1937 & 1,34 & 0,09 & 1981 & 1,61 & 0,06 \\
\hline 1938 & 1,37 & 0,86 & 1982 & 1,43 & $-1,44$ \\
\hline 1939 & 1,62 & 0,02 & 1983 & 1,67 & $-0,94$ \\
\hline 1940 & 1,31 & $-1,52$ & 1984 & 1,62 & $-0,14$ \\
\hline 1941 & 1,43 & $-1,44$ & 1985 & 1,57 & $-0,07$ \\
\hline 1943 & 1,49 & 0,35 & 1986 & 1,56 & $-0,32$ \\
\hline 1944 & 1,55 & $-0,27$ & 1987 & 1,46 & $-1,47$ \\
\hline 1945 & 1,71 & 0,42 & 1988 & 1,70 & 0,73 \\
\hline 1946 & 1,49 & $-0,79$ & 1989 & 1,51 & 0,61 \\
\hline
\end{tabular}


Tabela 2.16: Resultados dos testes da hipótese nula $\mathcal{H}_{0}: \gamma_{3}=0$ contra $\mathcal{H}_{1}: \gamma_{3} \neq 0$ - 'modelo I' - fremantle

\begin{tabular}{c|ccccc}
\hline \hline & & & & & \\
& $w$ & $W$ & $S_{R}$ & $S_{T}$ & $w^{*}$ \\
\hline estatística & 0,2053 & 0,2504 & 0,1710 & 0,2068 & 0,1611 \\
valor-p & 0,6505 & 0,6168 & 0,6792 & 0,6493 & 0,6881 \\
\hline \hline
\end{tabular}

Tabela 2.17: Resultados dos testes da hipótese nula $\mathcal{H}_{0}: \gamma_{2}=0$ contra $\mathcal{H}_{1}: \gamma_{2} \neq 0$ - 'modelo II' - fremantle

\begin{tabular}{c|ccccc}
\hline \hline & & & & & \\
& $w$ & $W$ & $S_{R}$ & $S_{T}$ & $w^{*}$ \\
\hline estatística & 2,1304 & 1,8813 & 2,4159 & 2,1307 & 1,9605 \\
valor-p & 0,1444 & 0,1702 & 0,1201 & 0,1444 & 0,1615 \\
\hline \hline
\end{tabular}

Tabela 2.18: Resultados dos testes da hipótese nula $\mathcal{H}_{0}: \beta_{3}=0$ contra $\mathcal{H}_{1}: \beta_{3} \neq 0$ - 'modelo III' - fremantle

\begin{tabular}{c|ccccc}
\hline \hline & & & & & \\
& $w$ & $W$ & $S_{R}$ & $S_{T}$ & $w^{*}$ \\
\hline estatística & 1,4806 & 0,2298 & 1,2885 & 0,5274 & 1,5309 \\
valor-p & 0,2237 & 0,6317 & 0,2563 & 0,4677 & 0,2160 \\
\hline \hline
\end{tabular}

Tabela 2.19: Resultados dos testes da hipótese nula $\mathcal{H}_{0}: \gamma_{1}=0$ contra $\mathcal{H}_{1}: \gamma_{1} \neq 0$ - 'modelo IV' - fremantle

\begin{tabular}{c|ccccc}
\hline \hline & & & & & \\
& $w$ & $W$ & $S_{R}$ & $S_{T}$ & $w^{*}$ \\
\hline estatística & 3,0002 & 3,4865 & 2,5292 & 2,9612 & 2,8931 \\
valor-p & 0,0833 & 0,0619 & 0,1118 & 0,0853 & 0,0890 \\
\hline \hline
\end{tabular}

Tabela 2.20: Resultados dos testes de hipótese - fremantle

\begin{tabular}{c|c|cccccc}
\hline \hline \multirow{2}{*}{ modelo } & $\mathcal{H}_{0}: \lambda=0 \times \mathcal{H}_{1}: \lambda \neq 0$ & & $w$ & $W$ & $S_{R}$ & $S_{T}$ & $w^{*}$ \\
\hline I & $\lambda=\gamma_{3}$ & estatística & 0,2053 & 0,2504 & 0,1710 & 0,2068 & 0,1611 \\
& & valor-p & 0,6505 & 0,6168 & 0,6792 & 0,6493 & 0,6881 \\
\hline II & $\lambda=\gamma_{2}$ & estatística & 2,1304 & 1,8813 & 2,4159 & 2,1307 & 1,9605 \\
& & valor-p & 0,1444 & 0,1702 & 0,1201 & 0,1444 & 0,1615 \\
\hline III & $\lambda=\beta_{3}$ & estatística & 1,4806 & 0,2298 & 1,2885 & 0,5274 & 1,5309 \\
& & valor-p & 0,2237 & 0,6317 & 0,2563 & 0,4677 & 0,2160 \\
\hline IV & $\lambda=\gamma_{1}$ & estatística & 3,0002 & 3,4865 & 2,5292 & 2,9612 & 2,8931 \\
& & valor-p & 0,0833 & 0,0619 & 0,1118 & 0,0853 & 0,0890 \\
\hline \hline
\end{tabular}


Tabela 2.21: Resultado do teste da hipótese nula $\mathcal{H}_{0}: \beta_{1}=0$ contra $\mathcal{H}_{1}: \beta_{1} \neq 0$ - decatlo

\begin{tabular}{c|ccccc}
\hline \hline & & & & & \\
& $w$ & $W$ & $S_{R}$ & $S_{T}$ & $w^{*}$ \\
\hline estatística & 4,0407 & 5,7161 & 2,8208 & 3,6293 & 2,6466 \\
valor-p & 0,0444 & 0,0168 & 0,0930 & 0,0568 & 0,1038 \\
\hline \hline
\end{tabular}




\section{Capítulo 3}

\section{Ajustes da razão de verossimilhança sinalizada em modelos de regressão valor extremo}

\subsection{Introdução}

Para fazer inferência acerca de um parâmetro unidimensional uma possibilidade é utilizar o teste da razão de verossimilhanças sinalizada. Este teste é comumente utilizado para testar hipóteses unilaterais.

Seja $\theta=\left(\beta^{\top}, \gamma^{\top}\right)^{\top}$ o vetor de parâmetros desconhecidos do modelo de regressão (2.1)-(2.3) de dimensões $k$ e $m$, respectivamente. No que segue, $\nu$ representa o parâmetro de interesse, neste caso unidimensional, e $\psi=\left(\psi_{1}, \ldots, \psi_{s}\right)^{\top}$ é o parâmetro de perturbação (note que $1+s=k+m$ ). A estatística da razão de verossimilhanças sinalizada é definida por

$$
R=R(\nu)=\operatorname{sgn}(\widehat{\nu}-\nu)[2(l(\widehat{\nu}, \widehat{\psi})-l(\nu, \widetilde{\psi}))]^{1 / 2},
$$

em que $\operatorname{sgn}(\widehat{\nu}-\nu)$ representa o sinal de $\widehat{\nu}-\nu,(\widehat{\nu}, \widehat{\psi})$ e $(\nu, \widetilde{\psi})$ são os estimadores de máxima verossimilhança irrestrito e restrito de $(\nu, \psi)$, respectivamente. O estimador $\widetilde{\psi}$ é obtido maximizando o logaritmo da função de verossimilhança $l(\nu, \psi)$ com relação a $\psi$ supondo $\nu$ fixado. A estatística (3.1) pode ser usada para testar as hipóteses $\mathcal{H}_{0}: \nu \leq \nu^{(0)}$ contra $\mathcal{H}_{1}: \nu>\nu^{(0)}, \mathcal{H}_{0}: \nu \geq \nu^{(0)}$ contra $\mathcal{H}_{1}: \nu<\nu^{(0)}$ e ainda, $\mathcal{H}_{0}: \nu=\nu^{(0)}$ contra $\mathcal{H}_{1}: \nu \neq \nu^{(0)}$, em que $\nu^{(0)}$ é uma constante fixada.

A estatística da razão de verossimilhanças sinalizada $(R)$ tem distribuição assintótica normal padrão sob a hipótese nula com erro de ordem $O\left(n^{-1 / 2}\right)$ (Severini, 2000, Seção 4.3 e 4.4). Em muitas situações, no entanto, a acurácia da aproximação pela distribuição normal é comprometida, por exemplo, quando o tamanho da amostra $n$ é pequeno ou moderado. Para aprimorar a acurácia da aproximação da distribuição da estatística da razão de verossimilhanças sinalizada pela distribuição normal, alguns ajustes foram propostos. Conforme citado em (Lemonte, 2010, Cap. 4), dentre os ajustes propostos estão Lawley (1956), DiCiccio (1984), McCullagh (1984), Jensen $(1986,1987)$ e a estatística da razão de verossimilhanças sinalizada ajustada $\left(R^{*}\right)$ proposta por Barndorff-Nielsen (1986).

A estatística $R^{*}$ tem distribuição assintótica normal padrão com erro de ordem $O\left(n^{-3 / 2}\right)$. Esta foi obtida em vários modelos particulares (ver Lemonte, 2010, Cap. 4), e ainda, mais recentemente, no modelo elíptico estrutural com erros nas variáveis por Melo \& Ferrari (2010). O ajuste da estatística $R^{*}$ depende da especificação de uma estatística ancilar. É possível explicitar uma estatística ancilar em modelos da famíla exponencial de distribuições e em modelos de transformação. No entanto, quando não se trata destas 
situações, é dificil apresentar uma estatística ancilar, o que acarretou o surgimento de várias propostas de aproximação para este ajuste que tratam de contornar o problema da inviabilidade desta especificação.

Algumas propostas de aproximação para o ajuste da estatística $R^{*}$ foram feitas por DiCiccio \& Martin (1993), Skovgaard (1996), Severini (1999) e Fraser et al. (1999). Para a dedução destas aproximações sugerimos Severini (2000, Cap. 7) e Lemonte (2010, Cap. 4).

Assim como o ajuste de Barndorff-Nielsen (1986) $R^{*}$, suas aproximações também têm sido obtidas em modelos particulares, por exemplo, Wu \& Wong (2004), Wu, Jiang \& Wei (2006), Wu, Wong \& Wei (2006), Wu \& Jiang (2007) e mais recentemente, no modelo de regressão Birnbaum-Saunders por Lemonte (2010).

Visando melhorar a qualidade da inferência sobre os parâmetros do modelo de regressão valor extremo obtemos, neste capítulo, o ajuste de Barndorff-Nielsen e as aproximações mencionadas acima para o caso linear homocesdástico. Para os modelos valor extremo linear heteroscedástico e não linear obteremos tais aproximações para $R^{*}$. Faremos um estudo comparativo através de simulação e apresentaremos uma aplicação num conjunto real de dados.

\subsection{Ajuste de Barndorff-Nielsen}

Barndorff-Nielsen $(1986,1991)$ propôs uma estatística da razão de verossimilhanças sinalizada ajustada $\left(R^{*}\right)$ que é definida em termos de derivadas do logaritmo da verossimilhança em relação ao espaço amostral, mais especificamente, em relação ao estimador de máxima verossimilhança do parâmetro $\theta(\widehat{\theta})$. Para obter tais derivadas é necessária a especificação de uma estatística ancilar (estatística cuja distribuição não depende dos parâmetros), digamos a, tal que $(\widehat{\theta}, \mathbf{a})$ seja uma estatística suficiente. Desta forma o logaritmo da função de verossimilhança pode ser escrito como $\ell(\theta ; \widehat{\theta}, \mathbf{a})$ e podemos obter suas derivadas em relação a $\widehat{\theta}$. A notação usual das derivadas em relação aos parâmetros do modelo são, por exemplo,

$$
l_{\theta}(\theta)=l_{\theta}(\theta ; \widehat{\theta}, \mathbf{a})=\frac{\partial l(\theta ; \widehat{\theta}, \mathbf{a})}{\partial \theta^{\top}}, \quad l_{\theta \theta}(\theta)=l_{\theta \theta}(\theta ; \widehat{\theta}, \mathbf{a})=\frac{\partial^{2} l(\theta ; \widehat{\theta}, \mathbf{a})}{\partial \theta \partial \theta^{\top}}, \quad \text { etc, }
$$

e as derivadas com relação a funções dos dados, neste caso $\widehat{\theta}$, são, por exemplo,

$$
l_{; \widehat{\theta}}(\theta)=l_{; \widehat{\theta}}(\theta ; \widehat{\theta}, \mathbf{a})=\frac{\partial l(\theta ; \widehat{\theta}, \mathbf{a})}{\partial \widehat{\theta}}, \quad l_{\theta ; \widehat{\theta}}(\theta)=l_{\theta ; \widehat{\theta}}(\theta ; \widehat{\theta}, \mathbf{a})=\frac{\partial^{2} l(\theta ; \widehat{\theta}, \mathbf{a})}{\partial \theta^{\top} \partial \widehat{\theta}}=\frac{\partial l_{\theta}(\theta ; \widehat{\theta}, \mathbf{a})}{\partial \widehat{\theta}}, \quad \text { etc. }
$$

Desta forma temos que $l_{\theta}(\theta)$ é um vetor coluna $(k+m) \times 1$ e $l_{; \hat{\theta}}(\theta)$ é um vetor linha $1 \times(k+m)$ e ainda $l_{\theta ; \theta}(\theta)$ e $l_{\theta ; \widehat{\theta}}(\theta)$ são matrizes $(k+m) \times(k+m)$.

A estatística da razão de verossimilhanças sinalizada ajustada de Barndorff-Nielsen é dada por

$$
R^{*}=R+\frac{1}{R} \ln \left(\left|\frac{U}{R}\right|\right)
$$

em que

$$
U=\frac{\left|\begin{array}{c}
l_{; \widehat{\theta}}(\widehat{\theta})-l_{; \widehat{\dot{\theta}}}(\widetilde{\theta}) \\
l_{\psi ; \widehat{\theta}}(\widetilde{\theta})
\end{array}\right|}{\left|\widetilde{J}_{\psi \psi}\right|^{1 / 2|\widehat{J}|^{1 / 2}}} .
$$


Aqui $l_{\psi ; \widehat{\theta}}(\widetilde{\theta})$ é obtida de $l_{\theta ; \widehat{\theta}}(\widetilde{\theta})$ excluindo-se a linha relativa ao parâmetro de interesse $\nu$. A estatística sinalizada modificada tem erro de aproximação pela distribuição normal padrão de ordem $O\left(n^{-3 / 2}\right)$. No caso particular uniparamétrico, ou seja, em que não há parâmetros de perturbação, temos que

$$
U=\left|l_{; \widehat{\theta}}(\widehat{\theta})-l_{; \widehat{\theta}}(\theta)\right||\widehat{J}|^{-1 / 2}
$$

Como o ajuste de Barndorff-Nielsen depende da especificação de uma estatística ancilar, em vários modelos não é possivel obter este ajuste explicitamente. Lemonte (2010, p. 53) lista uma série de artigos em que o ajuste foi obtido para diferentes modelos, em sua maioria da família exponencial.

É possível obter a estatística ajustada de Barndorff-Nielsen para um modelo de regressão linear homoscedástico, pois este é um caso particular de modelo de transformação. Num modelo de transformação toda estatística maximal invariante é uma estatística ancilar (ver Barndorff-Nielsen et al. , 1989, Cap. 8 e Barndorff-Nielsen, 1986). Segundo Severini (2000, Seção 1.3), num modelo de regressão linear homoscedástico, a estatística $a=(y-X \hat{\beta}) / \hat{\sigma}$ é maximal invariante e $(\hat{\beta}, \hat{\sigma}, a)$ é suficiente, isto é, é equivalente aos dados $y$. Portanto, num modelo de regressão linear homoscedástico, a estatística $a=(y-X \hat{\beta}) / \hat{\sigma}$ é ancilar. Ou, de outra forma, ver Apêndice D, Resultado 9.

O modelo de regressão valor extremo máximo linear com dispersão constante é um caso particular do modelo de regressão valor extremo máximo (2.1)-(2.3), em que $g$ é a função identidade, $\eta_{t}\left(x_{t}, \beta\right)=x_{t}^{\top} \beta, h$ é a função identidade e $\delta_{t}\left(z_{t}, \gamma\right)=z_{t}^{\top} \gamma=\sigma$. Para este modelo obtemos a estatística ajustada de BarndorffNielsen dada em (3.2), cujas quantidades são dadas por

$$
\begin{aligned}
& l_{; \widehat{\theta}}(\widehat{\theta})-l_{; \widehat{\theta}}(\widetilde{\theta})=\iota^{\top}\left(\widehat{\sigma}^{-1}\left(-\mathcal{I}+\widehat{\mathcal{Z}}^{\dagger}\right)-\widetilde{\sigma}^{-1}\left(-\mathcal{I}+\widetilde{\mathcal{Z}}^{\dagger}\right)\right)\left(\widehat{X} \quad \widehat{\mathcal{Z}}_{\iota}\right), \\
& l_{\theta ; \widehat{\theta}}(\widetilde{\theta})=\left.\frac{\partial^{2} \ell(\theta ; \widehat{\theta}, a)}{\partial \theta \partial \widehat{\theta}}\right|_{\widetilde{\theta}}=\widetilde{A}\left(\begin{array}{ll}
\widehat{X} & \widehat{\mathcal{Z}}_{\iota}
\end{array}\right)
\end{aligned}
$$

em que

$$
A=\sigma^{-2}\left(\begin{array}{c}
X^{\top} \mathcal{Z}^{\dagger} \\
\iota^{\top}\left(\mathcal{I}-\mathcal{Z}^{\dagger}+\mathcal{Z} \mathcal{Z}^{\dagger}\right)
\end{array}\right)
$$

e $X, Z, \mathcal{I}, T, H, \Phi, \mathcal{Z}, \mathcal{Z}^{\dagger}$ estão dadas na Seção 2.1 (ver Apêndice E). Podemos escrever

$$
U=\left|\left(\begin{array}{c}
\iota^{\top}\left(\widehat{\sigma}^{-1}\left(-\mathcal{I}+\widehat{\mathcal{Z}}^{\dagger}\right)-\widetilde{\sigma}^{-1}\left(-\mathcal{I}+\widetilde{\mathcal{Z}}^{\dagger}\right)\right) \\
\widetilde{A}_{\psi}
\end{array}\right)\left(\begin{array}{ll}
\widehat{X} & \widehat{\mathcal{Z}}_{\iota}
\end{array}\right)\right|\left|\widetilde{J}_{\psi \psi}\right|^{-1 / 2}|\widehat{J}|^{-1 / 2},
$$

em que $A_{\psi}$ é obtida de $A$ retirando-se a linha relativa ao parâmetro de interesse $\nu$.

No caso do modelo de regressão valor extremo mínimo (2.28)-(2.2)-(2.3), isto é, $y_{t} \sim V E_{\min }\left(\mu_{t}, \sigma_{t}\right)$, podemos obter a estatística $R^{*}$ considerando a variável $y_{t}^{*}=-y_{t}$ que tem distribuição valor extremo máximo com parâmetros $-\mu_{t}$ e $\sigma_{t}\left(-y_{t} \sim V E_{\max }\left(-\mu_{t}, \sigma_{t}\right)\right)$. Assim, calculamos $R^{*}$ para $y_{t}^{*}$, função de ligação do parâmetro de locação $g^{*}(x)=x$ e preditor do parâmetro de locação $\eta^{*}(\beta)=\left(-g^{-1} \circ \eta\right)(\beta)$. Como caso particular, para o modelo linear $\eta=X \beta$ e função de ligação identidade, definimos $\eta^{*}(\beta)=$ $-X \beta=(-X) \beta$. 


\subsection{Aproximações para o ajuste de Barndorff-Nielsen da estatística da razão de verossimi- lhanças sinalizada}

Algumas aproximações para as quantidades que envolvem derivadas em relação ao espaço amostral no ajuste de Barndorff-Nielsen $(1986,1991)$ da estatística da razão de verossimilhanças sinalizada foram propostas para quando não é possível especificar uma estatística ancilar. Nesta seção apresentamos algumas destas aproximações e suas versões para o modelo de regressão valor extremo máximo. Resultados para o modelo de regressão valor extremo mínimo (2.28)-(2.2)-(2.3) podem ser obtidos por uma reflexão da maneira usual, ver Beirlant et al. (2004, p. 46) e Seção (3.2).

\subsubsection{Ajuste de DiCiccio \& Martin (1993)}

DiCiccio \& Martin (1993) propuseram uma aproximação para o ajuste de Barndorff-Nielsen (1986, 1991) no caso em que o parâmetro de interesse é ortogonal aos parâmetros de perturbação. Sendo $\theta=(\nu, \psi)$ de dimensão $p, \nu$ o parâmetro de interesse unidimensional, $\psi$ o vetor de parâmetros de perturbação de dimensão $p-1$ e $I$ a matriz de informação de Fisher, os parâmetros $\nu$ e $\psi$ são ortogonais se $I_{\nu \psi}(\theta)=\mathrm{E}\left(-\partial^{2} \ell(\theta) / \partial \nu \partial \psi\right)=0_{1 \times(p-1)}$ e, neste caso, a matriz de informação de Fisher é bloco diagonal. Segundo Cox \& Reid $(1987,1993)$ quando o parâmetro de interesse é escalar sempre é possível obter uma parametrização ortogonal. Assim, sempre é possível obter a estatística ajustada de DiCiccio \& Martin (1993), denotada por $R_{0}^{*}$, que é definida por (3.2) com $U$ substituído por

$$
U_{0}=\widetilde{\ell}_{\nu}\left(\frac{\left|\widetilde{J}_{\psi \psi}\right|\left|\widehat{I}_{\nu \nu}\right|}{|\widehat{J}|\left|\widetilde{I}_{\nu \nu}\right|}\right)^{1 / 2}
$$

em que $\ell_{\nu}$ é a derivada do logaritmo da verossimilhança em relação ao parâmetro de interesse e $J$ e $I$ são as matrizes de informação observada e esperada, respectivamente. Além disso, $\quad \widehat{r}$ e $\sim$ indicam avaliação no estimador de máxima verossimilhança de $\theta(\widehat{\theta})$ e com restrição sob $\mathcal{H}_{0}(\widetilde{\theta})$ respectivamente. De acordo com DiCiccio \& Martin (1993), em geral, $R_{0}^{*}=R^{*}+O_{p}\left(n^{-1}\right)$, isto é, o erro de aproximação de $R^{*}$ por $R_{0}^{*}$ é de ordem $O_{p}\left(n^{-1}\right)$.

Considere agora o modelo (2.1)-(2.3). Sejam $\nu=\beta_{r}$ o parâmetro de interesse, $\beta_{(r)}$ o complemento de $\beta_{r}$ em relação a $\beta$ e $\theta_{(r)}=\left(\beta_{(r)}, \gamma\right)$ o complemento de $\beta_{r}$ em relação a $\theta$. Aqui, o complemento da coordenada $v_{i}$ do vetor $v=\left(v_{1}, \ldots, v_{i-1}, v_{i}, v_{i+1}, \ldots, v_{d}\right)$ de dimensão $d$ em relação a $v$, é o vetor $v_{(i)}=\left(v_{1}, \ldots, v_{i-1}, v_{i+1}, \ldots, v_{d}\right)$, isto é, é o vetor $v$ sem a coordenada $v_{i}$. Considere uma parametrização $\vartheta=\left(\beta_{r}, \vartheta_{(r)}\right), \vartheta_{(r)}=(\kappa, \tau)$ em que $\vartheta, \vartheta_{(r)}, \kappa$ e $\tau$ têm dimensões $(k+m),(k+m-1),(k-1)$ e $m$, respectivamente, de forma que o parâmetro $\beta_{r}$ seja ortogonal ao vetor dos demais parâmetros $\vartheta_{(r)}$. Para obter uma parametrização $\vartheta$ de forma que o parâmetro $\beta_{r}$ seja ortogonal ao vetor dos demais parâmetros, $\vartheta_{(r)}$, é necessário obter uma transformação de $\theta=\left(\beta_{r}, \theta_{(r)}\right)=\left(\beta_{r}, \beta_{(r)}, \gamma\right)$ em $\vartheta=\left(\beta_{r}, \vartheta_{(r)}\right)=\left(\beta_{r}, \kappa, \tau\right)$ de modo que $\mathrm{E}\left(-\partial^{2} \ell\left(\beta_{r}, \vartheta_{(r)}\right) / \partial \beta_{r} \partial \vartheta_{(r)}\right)=0$. A matriz de informação de Fisher dada em (2.14) pode ser escrita como

$$
I(\theta)=I\left(\beta_{r}, \theta_{(r)}\right)=I\left(\beta_{r}, \beta_{(r)}, \gamma\right)=\left(\begin{array}{ccc}
I_{\beta_{r} \beta_{r}} & I_{\beta_{r} \beta_{(r)}} & I_{\beta_{r} \gamma} \\
I_{\beta_{(r)} \beta_{r}} & I_{\beta_{(r)} \beta_{(r)}} & I_{\beta_{(r)} \gamma} \\
I_{\gamma \beta_{r}} & I_{\gamma \beta_{(r)}} & I_{\gamma \gamma}
\end{array}\right)
$$


em que

$$
\begin{gathered}
I_{\beta_{r} \beta_{r}}=\mathbf{x}_{\cdot r}^{\top} \Phi^{-1} T^{2} \Phi^{-1} \mathbf{x} \cdot r \\
I_{\beta_{r} \beta_{(r)}}=I_{\beta_{(r)} \beta_{r}}^{\top}=\mathbf{x}_{\cdot r}^{\top} \Phi^{-1} T^{2} \Phi^{-1} X_{(r)}, \\
I_{\beta_{r} \gamma}=I_{\gamma \beta_{r}}^{\top}=(\mathcal{E}-1) \mathbf{x}_{r}^{\top} \Phi^{-1} T H \Phi^{-1} Z, \\
I_{\beta_{(r)} \beta_{(r)}}=X_{(r)}^{\top} \Phi^{-1} T^{2} \Phi^{-1} X_{(r)}, \\
I_{\beta_{(r)} \gamma}=I_{\gamma \beta_{(r)}}^{\top}=(\mathcal{E}-1) X_{(r)}^{\top} \Phi^{-1} T H \Phi^{-1} Z, \\
I_{\gamma \gamma}=\left(1+\Gamma^{(2)}(2)\right) Z^{\top} \Phi^{-1} H^{2} \Phi^{-1} Z,
\end{gathered}
$$

$\mathbf{X}_{. r}=\left(x_{1 r}, \ldots, x_{n r}\right)^{\top}$ é a $r$-ésima coluna de $X$ e $X_{(r)}$ é a matriz $X$ sem a coluna $r$.

Segundo Cox \& Reid $(1987,1993)$ soluções do sistema de equações diferenciais

$$
\frac{\partial \theta_{(r)}}{\partial \beta_{r}}=-I_{\beta_{r} \theta_{(r)}} I_{\theta_{(r)} \theta_{(r)}}^{-1}
$$

produzem parametrizações ortogonais.

Sejam

$$
I_{\beta_{r} \theta_{(r)}}=\left(\begin{array}{ll}
I_{\beta_{r} \beta_{(r)}} & I_{\beta_{r} \gamma}
\end{array}\right), \quad I_{\theta_{(r)} \theta_{(r)}}=\left(\begin{array}{cc}
I_{\beta_{(r)} \beta_{(r)}} & I_{\beta_{(r)} \gamma} \\
I_{\gamma \beta_{(r)}} & I_{\gamma \gamma}
\end{array}\right), \quad I_{\theta_{(r)} \theta_{(r)}}^{-1}=\left(\begin{array}{cc}
I^{\beta_{(r)} \beta_{(r)}} & I^{\beta_{(r)} \gamma} \\
I^{\gamma \beta_{(r)}} & I^{\gamma \gamma}
\end{array}\right)
$$

$\mathrm{e}$

$$
\frac{\partial \theta_{(r)}}{\beta_{r}}=-\left(\begin{array}{ll}
A & B
\end{array}\right)^{\top},
$$

em que $A=I_{\beta_{r} \beta_{(r)}} I^{\beta_{(r)} \beta_{(r)}}+I_{\beta_{r} \gamma} I^{\gamma \beta_{(r)}}$ e $B=I_{\beta_{r} \beta_{(r)}} I^{\beta_{(r)} \gamma}+I_{\beta_{r} \gamma} I^{\gamma \gamma}$. Uma possível solução do sistema de equações diferenciais (3.3) é $\beta_{(r)}=\kappa-\beta_{r} A^{\top}$ e $\gamma=\tau-\beta_{r} B^{\top}$ e $\beta_{r}$ é ortogonal a $(\kappa, \tau)$.

Podemos reescrever o modelo (2.1)-(2.3) na parametrização ortogonal $\vartheta=\left(\beta_{r}, \kappa, \tau\right)$ e definir os componentes sistemáticos $\eta^{*}(\vartheta), \delta^{*}(\vartheta)$, o logaritmo da verossimilhança $\ell^{*}(\vartheta)$, as informações de Fisher observada $J^{*}(\vartheta)$ e esperada $I^{*}(\vartheta)$, para então, obter o ajuste de DiCiccio \& Martin (1993) $\left(R_{0}^{*}\right)$. A título de exemplo, obtemos o ajuste para duas possíveis configurações dos componentes sistemáticos do modelo.

Como primeiro exemplo, considere o modelo linear (2.1) com componentes sistemáticos da localização e dispersão dados, respectivamente, por

$$
g\left(\mu_{t}\right)=\eta_{t}=\eta\left(x_{t}, \beta\right)=\mathbf{x}_{t} \beta
$$

e

$$
h\left(\sigma_{t}\right)=\delta_{t}=\delta\left(z_{t}, \gamma\right)=\mathbf{z}_{t} \gamma .
$$

Sejam $\mathbf{x}_{t}=\left(x_{t 1}, \ldots, x_{t k}\right)$ a linha $t$ de $X, \mathbf{z}_{t}=\left(z_{t 1}, \ldots, z_{t m}\right)$ a linha $t$ de $Z, \mathbf{x}_{t(r)}=$ $\left(x_{t 1}, \ldots, x_{t(r-1)}, x_{t(r+1)}, \ldots, x_{t k}\right)$ a linha $t$ de $X$ sem a coluna $r$ e $\mathbf{x}_{. r}$ e $X_{(r)}$ como definidos anterior- 
mente. Temos que

$$
\eta_{t}(\theta)=x_{t r} \beta_{r}+\mathbf{x}_{t(r)} \beta_{(r)}=x_{t r} \beta_{r}+\mathbf{x}_{t(r)}\left(\kappa-\beta_{r} A^{\top}\right)=\left(x_{t r}-\mathbf{x}_{t(r)} A^{\top}\right) \beta_{r}+\mathbf{x}_{t(r)} \kappa \equiv \eta_{t}^{*}(\vartheta)
$$

e

$$
\delta_{t}=\mathbf{z}_{t} \cdot \tau-\beta_{r} \mathbf{z}_{t} \cdot B^{\top} \equiv \delta_{t}^{*}(\vartheta)
$$

Segue que

$$
\begin{gathered}
\frac{\partial \eta_{t}^{*}(\vartheta)}{\partial \beta_{r}}=x_{t r}-\mathbf{x}_{t(r)} A^{\top}, \\
\frac{\partial \delta_{t}^{*}(\vartheta)}{\partial \beta_{r}}=-\mathbf{z}_{t} \cdot B^{\top} .
\end{gathered}
$$

Sejam $\ell^{*}(\vartheta), J^{*}(\vartheta)$ e $I^{*}(\vartheta)$ o logaritmo da verossimilhança e as informações de Fisher observada e esperada, respectivamente, do modelo (2.1)-(2.3) reparametrizado. Para esta parametrização obtemos

$$
\begin{array}{cl}
\ell_{\beta_{r}}^{*}(\vartheta)=\frac{\partial \ell^{*}(\vartheta)}{\partial \beta_{r}}=\iota^{\top}\left(\Phi^{-1} T\left(\mathcal{I}-\mathcal{Z}^{\dagger}\right) \mathbf{v}_{1}+\Phi^{-1} H\left(-\mathcal{I}+\mathcal{Z}-\mathcal{Z} \mathcal{Z}^{\dagger}\right) \mathbf{v}_{2}\right), \\
J_{\beta_{r} \beta_{r}}^{*}=\mathbf{v}_{1}^{\top} V_{\beta \beta} \mathbf{v}_{1}+2 \mathbf{v}_{1}^{\top} V_{\beta \gamma} \mathbf{v}_{2}+\mathbf{v}_{2}^{\top} V_{\gamma \gamma} \mathbf{v}_{2}, & J_{\kappa \kappa}^{*}=X_{(r)}^{\top} V_{\beta \beta} X_{(r)}, \\
J_{\beta_{r} \kappa}^{*}=J_{\kappa \beta_{r}}^{\top}=\mathbf{v}_{1}^{\top} V_{\beta \beta} X_{(r)}+\mathbf{v}_{2}^{\top} V_{\beta \gamma} X_{(r)}, & J_{\kappa \tau}^{*}=J_{\tau \kappa}^{\top}=X_{(r)}^{\top} V_{\beta \gamma} Z \\
J_{\beta_{r} \tau}^{*}=J_{\tau \beta_{r}}^{\top}=\mathbf{v}_{1}^{\top} V_{\beta \gamma} Z+\mathbf{v}_{2}^{\top} V_{\gamma \gamma} Z, & J_{\tau \tau}^{*}=Z^{\top} V_{\gamma \gamma} Z
\end{array}
$$

e

$$
I_{\beta_{r} \beta_{r}}^{*}=\mathbf{v}_{1}^{\top} \Phi^{-1} T^{2} \Phi^{-1} \mathbf{v}_{1}+2(\mathcal{E}-1) \mathbf{v}_{1}^{\top} \Phi^{-1} T H \Phi^{-1} \mathbf{v}_{2}+\left(1+\Gamma^{(2)}(2)\right) \mathbf{v}_{2}^{\top} \Phi^{-1} H^{2} \Phi^{-1} \mathbf{v}_{2},
$$

em que $\mathbf{v}_{1}=\left(\mathbf{x}_{\cdot r}-X_{(r)} A^{\top}\right), \mathbf{v}_{2}=-\left(Z B^{\top}\right), V_{\beta \beta}=\Phi^{-1} T\left(\mathcal{Z}^{\dagger} \Phi^{-1}+\left(\mathcal{I}-\mathcal{Z}^{\dagger}\right) S T\right) T, V_{\beta \gamma}=\Phi^{-1} T(\mathcal{I}-$ $\left.\mathcal{Z}^{\dagger}+\mathcal{Z} \mathcal{Z}^{\dagger}\right) H \Phi^{-1}$ e $V_{\gamma \gamma}=\Phi^{-1} H\left(\left(-\mathcal{I}+2 \mathcal{Z}-2 \mathcal{Z} \mathcal{Z}^{\dagger}+\mathcal{Z}^{2} \mathcal{Z}^{\dagger}\right) \Phi^{-1}+\left(-\mathcal{I}+\mathcal{Z}-\mathcal{Z} \mathcal{Z}^{\dagger}\right) Q H\right) H($ ver Apêndice E).

Como segundo exemplo, considere o modelo não linear (2.1) com componentes sistemáticos da localização e dispersão dados, respectivamente, por

$$
g\left(\mu_{t}\right)=\mu_{t}=\eta_{t}=\eta\left(\mathbf{x}_{t}, \beta\right)=\beta_{0}+\beta_{1} x_{t 1}+\exp \left(\beta_{2} x_{t 2}\right)
$$

e

$$
h\left(\phi_{t}\right)=\phi_{t}=\delta_{t}=\delta\left(\mathbf{z}_{t}, \gamma\right)=\mathbf{z}_{t} \gamma=\gamma_{0}
$$

Neste caso particular, temos que $\theta=\left(\beta_{0}, \beta_{1}, \beta_{2}, \gamma_{0}\right), X$ é uma matriz $n \times 3$, cuja $t$-ésima linha é $\left(1, x_{t 1}, x_{t 2} \exp \left(\beta_{2} x_{t 2}\right)\right), Z=\iota, T=\mathcal{I}, H=\mathcal{I}, S$ e $Q$ são matrizes nulas, $\dot{X}=\partial^{2} \eta / \partial \beta \partial \beta^{\top}$ é tal que $\partial^{2} \eta_{t} / \partial \beta_{2}^{2}=x_{t 2}^{2} \exp \left(\beta_{2} x_{t 2}\right)$ para $t=1, \ldots, n$ e zero caso contrário, $\dot{Z}=\partial^{2} \delta / \partial \gamma \partial \gamma^{\top}$ é o array nulo $n \times m \times m$. O produto 'colchete' da matriz $\left[\iota^{\top}\left(\mathcal{I}-\mathcal{Z}^{\dagger}\right) T \Phi^{-1}\right]$ de dimensão $1 \times n$, pelo array $\dot{X}$ de 
dimensão $n \times 3 \times 3$, é um array $1 \times 3 \times 3$, isto é, uma matriz $3 \times 3$, cujo elemento $(i, j)$ é

$$
\sum_{t=1}^{n}\left\{\left(\frac{1}{\phi_{t}}-\frac{1}{\phi_{t}} \exp \left(-\frac{y_{t}-\mu_{t}}{\phi_{t}}\right)\right) \frac{1}{g^{\prime}\left(\mu_{t}\right)} x_{t 2}^{2} \exp \left(\beta_{2} x_{t 2}\right)\right\}
$$

se $(i, j)=(3,3)$ e zero caso contrário. Como, neste caso particular, $\theta=\left(\beta_{0}, \beta_{1}, \beta_{2}, \gamma\right)=\left(\beta_{(2)}, \beta_{2}, \gamma\right)$ e $\vartheta=\left(\kappa_{0}, \kappa_{1}, \beta_{2}, \tau\right)=\left(\kappa, \beta_{2}, \tau\right)$, uma possível solução do sistema de equações diferenciais (3.3) é $\beta_{(2)}=$ $\kappa-\beta_{2} A^{\top}$ e $\gamma=\tau-\beta_{2} B^{\top}$. Então

$\eta_{t}(\theta)=\mathbf{x}_{t(2)} \beta_{(2)}+\exp \left(\beta_{2} x_{t 2}\right)=\mathbf{x}_{t(2)}\left(\kappa-\beta_{2} A^{\top}\right)+\exp \left(\beta_{2} x_{t 2}\right)=\mathbf{x}_{t(2)} \kappa+\exp \left(\beta_{2} x_{t 2}\right)-\beta_{2} \mathbf{x}_{t(2)} A^{\top} \equiv \eta_{t}^{*}(\vartheta)$

$\mathrm{e}$

$$
\delta_{t}(\theta)=\mathbf{z}_{t} \cdot \gamma=\mathbf{z}_{t \cdot}\left(\tau-\beta_{2} B^{\top}\right)=\mathbf{z}_{t} \cdot \tau-\beta_{2} \mathbf{z}_{t} \cdot B^{\top} \equiv \delta_{t}^{*}(\vartheta)
$$

Segue que

$$
\begin{gathered}
\frac{\partial \eta_{t}^{*}(\vartheta)}{\partial \beta_{2}}=x_{t 2} \exp \left(\beta_{2} x_{t 2}\right)-\mathbf{x}_{t(2)} A^{\top}, \\
\frac{\partial \delta_{t}^{*}(\vartheta)}{\partial \beta_{2}}=-\mathbf{z}_{t} \cdot B^{\top}
\end{gathered}
$$

e

$$
\frac{\partial^{2} \eta_{t}^{*}(\vartheta)}{\partial \beta_{2}^{2}}=x_{t 2}^{2} \exp \left(\beta_{2} x_{t 2}\right)
$$

Para esta parametrização obtemos que

$$
X^{*}=\left(\begin{array}{ll}
X_{(r)} & \mathbf{v}_{1}
\end{array}\right)
$$

é uma matriz $n \times 3$, cuja $t$-ésima linha é $\left(1, x_{t 1}, x_{t 2} \exp \left(\beta_{2} x_{t 2}\right)-\beta_{2} x_{t 2} A^{\top}\right)$,

$$
Z^{*}=\left(\begin{array}{ll}
Z & \mathbf{v}_{2}
\end{array}\right)
$$

é uma matriz $n \times(m+2)$, cuja $t$-ésima linha é $\left(\mathbf{z}_{t},,-\mathbf{z}_{t} B^{\top}\right)$,

$$
J_{\beta_{r} \beta_{r}}^{*}=\mathbf{v}_{1}^{\top} V_{\beta \beta} \mathbf{v}_{1}^{\top}+2 \mathbf{v}_{1}^{\top} V_{\beta \gamma} \mathbf{v}_{2}-\iota^{\top}\left(\mathcal{I}-\mathcal{Z}^{\dagger}\right) T \Phi^{-1} \mathbf{v}_{3}+\mathbf{v}_{2}^{\top} V_{\gamma \gamma} \mathbf{v}_{2}
$$

em que $\mathbf{v}_{1}=\left(x_{12} \exp \left(\beta_{2} x_{12}\right)-\mathbf{x}_{1(2)} A^{\top}, \ldots, x_{n 2} \exp \left(\beta_{2} x_{n 2}\right)-\mathbf{x}_{n(2)} A^{\top}\right)^{\top}, \mathbf{v}_{2}=-Z B^{\top}, \mathbf{v}_{3}=$ $\left(x_{12}^{2} \exp \left(\beta_{2} x_{12}\right), \ldots, x_{n 2}^{2} \exp \left(\beta_{2} x_{n 2}\right)\right)^{\top}$, e as outras quantidades são iguais às do modelo linear com $\mathbf{v}_{1}$ e $\mathbf{v}_{2}$ como definidos acima para o modelo não linear (ver Apêndice E).

\subsubsection{Ajuste de Skovgaard}

A estatística ajustada de Skovgaard (1996), denotada por $\bar{R}^{*}$, é baseada em covariâncias e é definida por (3.2) com $U$ substituído por

$$
\bar{U}=\left|\left(\begin{array}{c}
q^{\top} \\
\Upsilon_{\psi}
\end{array}\right)\right|\left|\widehat{I}^{-1}\right| \widehat{J}^{1 / 2}\left|\widetilde{J}_{\psi \psi}\right|^{-1 / 2}
$$


em que $q$, covariância entre o logaritmo da razão de verossimilhanças irrestrita e restrita e o vetor escore calculado no estimador de máxima verossimilhança irrestrito, é o vetor definido em (2.21), $\Upsilon$, covariância entre os vetores escore calculados nos estimadores de máxima verossimilhança irrestrito e restrito, é a matriz definida em (2.22), $\Upsilon_{\psi}$ é a matriz $\Upsilon$ sem a linha correspondente ao parâmetro de interesse $\nu$ e $J$ e $I$ são as matrizes de informação de Fisher observada e esperada. De acordo com Skovgaard (1996) e Severini (2000, Cap. 7), $\bar{R}^{*}=R^{*}+O_{p}\left(n^{-1}\right)$.

Para o modelo de regressão valor extremo máximo (2.1)-(2.3), obtemos $\bar{U}$ substituindo $q$ e $\Upsilon$ na equação acima por suas expressões relativas ao modelo apresentadas em (2.23), (2.24), respectivamente. As matrizes de informação observada e esperada do modelo estão dadas em (2.10) e (2.14).

\subsubsection{Ajuste de Severini}

A estatística ajustada de Severini (1999), denotada por $\widehat{R}^{*}$, é baseada em covariâncias empíricas, e é definida por (3.2) com $U$ substituído por

$$
\widehat{U}=\left|\left(\begin{array}{c}
\widehat{q}^{\top} \\
\widehat{\Upsilon}_{\psi}
\end{array}\right)\right|\left|\widehat{I}^{-1}\right| \widehat{J}^{1 / 2}\left|\widetilde{J}_{\psi \psi}\right|^{-1 / 2}
$$

em que as quantidades $\widehat{q}$ e $\widehat{\Upsilon}$, estimadores de $q$ dado em (2.21) e $\Upsilon$ dado em (2.22), são definidas por

$$
\widehat{q}=\sum_{t=1}^{n}\left(\ell_{t}(\widehat{\theta})-\ell_{t}(\widetilde{\theta})\right) \frac{\partial \ell_{t}(\widehat{\theta})}{\partial \theta^{\top}}
$$

$\mathrm{e}$

$$
\widehat{\Upsilon}=\sum_{t=1}^{n} \frac{\partial \ell_{t}(\widetilde{\theta})}{\partial \theta} \frac{\partial \ell_{t}(\widehat{\theta})}{\partial \theta^{\top}},
$$

$J$ e $I$ são as matrizes de informação observada e esperada, respectivamente, e $J_{\psi \psi}$ é obtida de $J$ retirando-se a linha e a coluna relativa ao parâmetro de interesse $\nu$.

Para o modelo de regressão valor extremo máximo (2.1)-(2.3) obtemos

$$
\widehat{q}=\left(\begin{array}{c}
\widehat{X} \widehat{\Phi}^{-1} \widehat{T}(\widehat{\mathfrak{L}}-\widetilde{\mathfrak{L}})\left(\iota-\widehat{\mathfrak{z}}^{\dagger}\right) \\
\widehat{Z} \widehat{\Phi}^{-1} \widehat{H}(\widehat{\mathfrak{L}}-\widetilde{\mathfrak{L}})\left(-\iota+\widehat{\mathfrak{z}}-\widehat{\mathcal{Z}} \widehat{\mathfrak{z}}^{\dagger}\right)
\end{array}\right)
$$

$\mathrm{e}$

$$
\widehat{\Upsilon}=\left[\begin{array}{ll}
\widehat{\Upsilon}_{\beta \beta} & \widehat{\Upsilon}_{\beta \gamma} \\
\widehat{\Upsilon}_{\gamma \beta} & \widehat{\Upsilon}_{\gamma \gamma}
\end{array}\right]
$$

em que

$$
\begin{aligned}
& \widehat{\Upsilon}_{\beta \beta}=\widetilde{X}^{\top} \widetilde{\Phi}^{-1} \widetilde{T}\left(\mathcal{I}-\widetilde{\mathcal{Z}}^{\dagger}\right)\left(\mathcal{I}-\widehat{\mathcal{Z}}^{\dagger}\right) \widehat{\Phi}^{-1} \widehat{T} \widehat{X}, \\
& \widehat{\Upsilon}_{\beta \gamma}=\widetilde{X}^{\top} \widetilde{\Phi}^{-1} \widetilde{T}\left(\mathcal{I}-\widetilde{\mathcal{Z}}^{\dagger}\right)\left(-\mathcal{I}+\widehat{\mathcal{Z}}-\widehat{\mathcal{Z}} \widehat{\mathcal{Z}}^{\dagger}\right) \widehat{\Phi}^{-1} \widehat{H} \widehat{Z}, \\
& \widehat{\Upsilon}_{\gamma \beta}=\widetilde{Z} \widetilde{H} \widetilde{\Phi}^{-1}\left(-\mathcal{I}+\widetilde{\mathcal{Z}}-\widetilde{\mathcal{Z}} \widetilde{\mathcal{Z}}^{\dagger}\right)\left(\mathcal{I}-\widehat{\mathcal{Z}}^{\dagger}\right) \widehat{\Phi}^{-1} \widehat{T} \widehat{X}, \\
& \widehat{\Upsilon}_{\gamma \gamma}=\widetilde{Z} \widetilde{H} \widetilde{\Phi}^{-1}\left(-\mathcal{I}+\widetilde{\mathcal{Z}}-\widetilde{\mathcal{Z}} \widetilde{\mathcal{Z}}^{\dagger}\right)\left(-\mathcal{I}+\widehat{\mathcal{Z}}-\widehat{\mathcal{Z}} \widehat{\mathcal{Z}}^{\dagger}\right) \widehat{\Phi}^{-1} \widehat{H} \widehat{Z},
\end{aligned}
$$


$\mathfrak{L}=\operatorname{diag}\left\{\ell_{1}, \ldots, \ell_{n}\right\}, \ell_{t}$ como definido em (2.5) e as outras quantidades como definidas na Seção 2.1 (ver Apêndice E). De acordo com Severini (1999), $\widehat{R}^{*}=R^{*}+O_{p}\left(n^{-1}\right)$.

\subsubsection{Ajuste de Fraser, Reid \& Wu}

A estatística ajustada de Fraser, Reid \& Wu (Fraser et al., 1999), denotada por $\widetilde{R}^{*}$, baseia-se numa estatística aproximadamente ancilar e é definida por (3.2) com $U$ substituído por

$$
\widetilde{U}=\frac{\left|\begin{array}{c}
\tilde{\ell}_{; \widehat{\theta}}(\widehat{\theta})-\tilde{\ell}_{; \widehat{\theta}}(\widetilde{\theta}) \\
\tilde{\ell}_{\psi ; \widehat{\theta}}(\tilde{\theta})
\end{array}\right|}{\left|\widetilde{J}_{\psi \psi}\right|^{1 / 2|\widehat{J}|^{1 / 2}}},
$$

em que

$$
\tilde{\ell}_{; \widehat{\theta}}(\widehat{\theta})-\tilde{\ell}_{; \widehat{\theta}}(\widetilde{\theta})=\left(\ell_{; y}(\widehat{\theta})-\ell_{; y}(\widetilde{\theta})\right) \widehat{V}\left(\ell_{\theta ; y}(\widehat{\theta}) \widehat{V}\right)^{-1} \widehat{J}
$$

é um vetor $1 \times(k+m)$,

$$
\tilde{\ell}_{\theta ; \widehat{\theta}}(\widetilde{\theta})=\ell_{\theta ; y}(\widetilde{\theta}) \widehat{V}\left(\ell_{\theta ; y}(\widehat{\theta}) \widehat{V}\right)^{-1} \widehat{J}
$$

é uma matriz $(k+m) \times(k+m), \ell_{; y}$ é a derivada do logaritmo da verossimilhança em relação a $y$, $\ell_{\theta ; y}$ é a segunda derivada em relação a $\theta$ e $y$, e $\widehat{V}$ é uma matriz $n \times(k+m)$ cujo elemento $(i, j)$ é $\left.\left(-\left(\partial F\left(y_{i}, \theta\right) / \partial \theta_{j}\right) / f\left(y_{i}, \theta\right)\right)\right|_{\theta=\widehat{\theta}}, F$ é a função de distribuição acumulada do modelo, $f$ a função densidade de probabilidade e $J$ a informação observada.

Obtemos para o modelo de regressão valor extremo máximo (2.1)-(2.3):

$$
\tilde{\ell}_{; \widehat{\theta}}(\widehat{\theta})-\tilde{\ell}_{; \widehat{\theta}}(\widetilde{\theta})=\iota\left(\widehat{\Phi}^{-1}\left(-\mathcal{I}+\widehat{\mathcal{Z}}^{\dagger}\right)-\widetilde{\Phi}^{-1}\left(-\mathcal{I}+\widetilde{Z}^{\dagger}\right)\right)(\widehat{T} \widehat{X} \quad \widehat{\mathcal{Z}} \widehat{H} \widehat{Z})(\widehat{A}(\widehat{T} \widehat{X} \quad \widehat{\mathcal{Z}} \widehat{H} \widehat{Z}))^{-1} \widehat{J}
$$

e

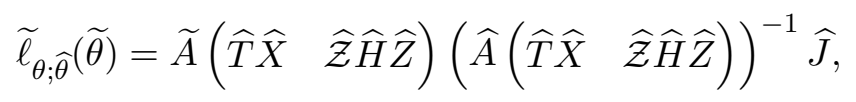

em que

$$
A=\left(\begin{array}{c}
X^{\top} T \Phi^{-2} \mathcal{Z}^{\dagger} \\
Z^{\top} H \Phi^{-2}\left(\mathcal{I}-\mathcal{Z}^{\dagger}+\mathcal{Z} \mathcal{Z}^{\dagger}\right)
\end{array}\right)
$$

$\widehat{A}=A(\widehat{\theta}), \widetilde{A}=A(\widetilde{\theta}), \widetilde{l}_{\psi ; \widehat{\theta}}(\widetilde{\theta})$ é obtido de $\widetilde{l}_{\theta ; \widehat{\theta}}(\widetilde{\theta})$ excluindo-se a linha relativa ao parâmetro de interesse $\nu$ e as outras quantidades são como definidas na Seção 2.1 (ver Apêndice E). Podemos escrever

$$
\widetilde{U}=\left|\left(\begin{array}{c}
\iota\left(\widehat{\Phi}^{-1}\left(-\mathcal{I}+\widehat{\mathcal{Z}}^{\dagger}\right)-\widetilde{\Phi}^{-1}\left(-\mathcal{I}+\widetilde{\mathcal{Z}}^{\dagger}\right)\right) \\
\widetilde{A}_{\psi}
\end{array}\right)\left(\begin{array}{ll}
\widehat{T} \widehat{X} & \widehat{\mathcal{Z}} \widehat{H} \widehat{Z}
\end{array}\right)\left(\begin{array}{cc}
\widehat{A}(\widehat{T} \widehat{X} & \widehat{\mathcal{Z}} \widehat{H} \widehat{Z})
\end{array}\right)^{-1}\right|\left|\widetilde{J}_{\psi \psi}\right|^{-1 / 2}|\widehat{J}|^{1 / 2} .
$$

De acordo com Fraser et al. (1999), $\widetilde{R}^{*}=R^{*}+O_{p}\left(n^{-3 / 2}\right)$. A ordem do erro de aproximação de $\widetilde{R}^{*}$ é menor do que a ordem dos erros das aproximações obtidas com os ajustes $R_{0}^{*}, \bar{R}^{*}$ e $\widehat{R}^{*}$, que são de ordem $O_{p}\left(n^{-1}\right)$. 


\subsection{Simulações - valor extremo máximo}

Apresentamos a seguir o comportamento dos testes da razão de verossimilhanças sinalizada $(R)$, da razão de verossimilhanças sinalizada ajustada de Barndorff-Nielsen (1986) $\left(R^{*}\right)$ e dos testes baseados nas aproximações para $R^{*}$ propostas por DiCiccio \& Martin (1993) $\left(R_{0}^{*}\right)$, Skovgaard (1996) $\left(\bar{R}^{*}\right)$, Severini (1999) $\left(\widehat{R}^{*}\right)$ e Fraser et al. (1999) $\left(\widetilde{R}^{*}\right)$, no modelo de regressão valor extremo máximo para amostras pequenas e de tamanho moderado, através de resultados das simulações de Monte Carlo. Os parâmetros são estimados através da maximização do logaritmo da função de verossimilhança utilizando o método de otimização não-linear quasi-Newton BFGS com primeiras derivadas analíticas desenvolvido por Broyden, Fletcher, Goldfarf \& Shanno (ver, por exemplo, Press et al., 1992), implementado na linguagem de programação Ox (Doornik, 2009) na função MaxBFGS.

Nas Seções 3.4.1, 3.4.2 e 3.4.3 consideramos os modelos de regressão valor extremo máximo linear com dispersão constante, dispersão variável e não linear com dispersão constante, respectivamente. O número de réplicas de Monte Carlo usado para comparação de tamanho dos testes foi 10.000 e os níveis nominais dos testes foram $\alpha=10 \%, 5 \%$ e $1 \%$. Se o número de elementos da amostra é pequeno ou moderado, os resultados da simulação mostram que os testes têm tamanhos distintos quando a distribuição assintótica normal é usada como aproximação. Para avaliar o poder destes testes, deve-se garantir que tenham o tamanho correto sob a hipótese nula. A fim de contornar esta dificuldade, foram simuladas 500.000 amostras de Monte Carlo sob cada hipótese nula estudada, para obter a distribuição empírica das estatísticas e estimar o valor crítico exato para cada teste para o nível nominal escolhido. Foram usados como valores críticos exatos, para avaliar o poder dos testes, os quantis amostrais de ordem 90\% das distribuições empíricas das estatísticas.

\subsubsection{Modelo de regressão valor extremo máximo linear com dispersão constante}

Considera-se o modelo (2.1) com parâmetro de dispersão constante e com componente sistemático da localização dado por

$$
\mu_{t}=\beta_{1}+\beta_{2} x_{t 2}+\beta_{3} x_{t 3}+\beta_{4} x_{t 4}+\beta_{5} x_{t 5},
$$

o qual denominamos de 'modelo 1'. Considera-se a hipótese nula $\mathcal{H}_{0}: \beta_{2} \leq 0$ a ser testada contra a hipótese alternativa $\mathcal{H}_{1}: \beta_{2}>0$, Assume-se $\beta_{1}=1, \beta_{2}=0, \beta_{3}=1, \beta_{4}=6, \beta_{5}=-3$ e $\sigma=1$. Os valores das covariadas foram obtidos aleatoriamente de uma distribuição $\mathcal{U}(-0,5,0,5)$. Os tamanhos das amostras utilizados são 15, 20, 30 e 40.

A Tabela 3.1 apresenta as taxas de rejeição nulas dos seis testes. Podemos notar que o teste da razão de verossimilhanças sinalizada é liberal, especialmente quando a amostra é pequena. Por exemplo, para $n=15$ e $\alpha=5 \%$, a taxa de rejeição nula é $8,4 \%$ enquanto as dos testes ajustados $R^{*}$ (Barndorff-Nielsen), $R_{0}^{*}$ (DiCiccio-Martin) e $\widetilde{R}^{*}$ (Fraser-Reid-Wu) são iguais a 4,9\%. O teste ajustado $\bar{R}^{*}$ (Skovgaard) é conservador, mas a taxa de rejeição nula está próxima do nível nominal sendo 4,2\% para o nível nominal de 5\% e $n=15$. O teste ajustado $\widehat{R}^{*}$ (Severini) também é conservador, mas para $n=30$ as taxas de rejeição estão bastante próximas dos níveis nominais $10 \%, 5 \%$ e $1 \%$, sendo, respectivamente, $10,1 \%, 4,8 \%$ e $0,9 \%$. Neste caso os testes $R^{*}$ (Barndorff-Nielsen), $R_{0}^{*}$ (DiCiccio-Martin) e $\widetilde{R}^{*}$ (Fraser-Reid-Wu) são os que se comportam melhor em relação a probabilidade de erro do tipo I quando o tamanho da amostra é pequeno, $n=15$ 
e $n=20$, e os testes $\bar{R}^{*}$ (Skovgaard) e $\widehat{R}^{*}$ (Severini) são os que se comportam melhor em relação a probabilidade de erro do tipo I quando o tamanho da amostra é moderado, $n=30$.

Tabela 3.1: Taxas de rejeição nula (\%); modelo 1

\begin{tabular}{|c|c|c|c|c|c|c|c|c|c|c|c|c|c|c|c|c|c|c|}
\hline \multirow[b]{2}{*}{$n$} & \multicolumn{6}{|c|}{$\alpha=10 \%$} & \multicolumn{6}{|c|}{$\alpha=5 \%$} & \multicolumn{6}{|c|}{$\alpha=1 \%$} \\
\hline & $R$ & $R_{0}^{*}$ & $\bar{R}^{*}$ & $\widehat{R}^{*}$ & $\widetilde{R}^{*}$ & $R^{*}$ & $R$ & $R_{0}^{*}$ & $\bar{R}^{*}$ & $\widehat{R}^{*}$ & $\widetilde{R}^{*}$ & $R^{*}$ & $R$ & $R_{0}^{*}$ & $\bar{R}^{*}$ & $\widehat{R}^{*}$ & $\widetilde{R}^{*}$ & $R^{*}$ \\
\hline 15 & 13,8 & 10,0 & 9,0 & 6,8 & 10,0 & 10,0 & 8,4 & 4,9 & 4,2 & 3,3 & 4,9 & 4,9 & 2,7 & 1,0 & 0,7 & 0,6 & 1,0 & 1,0 \\
\hline 20 & 13,5 & 9,9 & 8,8 & 8,3 & 9,9 & 9,9 & 8,1 & 5,0 & 4,0 & 3,8 & 5,0 & 5,0 & 2,3 & 1,1 & 0,8 & 0,7 & 1,1 & 1,1 \\
\hline 30 & 13,7 & 10,8 & 10,1 & 10,1 & 10,8 & 10,8 & 7,7 & 5,2 & 4,8 & 4,8 & 5,2 & 5,2 & 2,2 & 1,2 & 0,9 & 1,0 & 1,2 & 1,2 \\
\hline 40 & 12,2 & 9,6 & 8,8 & 9,7 & 9,6 & 9,6 & 6,7 & 4,8 & 4,3 & 4,8 & 4,8 & 4,8 & 1,7 & 1,2 & 1,0 & 1,1 & 1,2 & 1,2 \\
\hline 100 & 10,5 & 9,6 & 9,2 & 9,5 & 9,6 & 9,6 & 5,2 & 4,7 & 4,6 & 4,8 & 4,7 & 4,7 & 1,2 & 0,9 & 0,8 & 0,9 & 0,9 & 0,9 \\
\hline 200 & 10,5 & 10,1 & 10,0 & 10,2 & 10,1 & 10,1 & 5,3 & 5,0 & 4,8 & 5,0 & 5,0 & 5,0 & 1,2 & 1,0 & 0,9 & 1,1 & 1,0 & 1,0 \\
\hline
\end{tabular}

A Figura 3.1 apresenta gráficos de discrepâncias relativas de valor-p. Discrepância relativa de valor$\mathrm{p}$ é a diferença entre o valor-p exato e o assintótico dividido pelo último. A característica marcante da Figura 3.1 é que a discrepância relativa de valor-p do teste da razão de verossimilhanças sinalizada na cauda da distribuição assíntota normal (valor-p assintótico pequeno) é muito grande, isto é, o teste usual rejeita a hipótese nula com uma frequência muito maior do que o nível nominal quando este é menor do que $10 \%$, para os diferentes tamanhos de amostra apresentados. Os testes baseados nas estatísticas ajustadas têm um desempenho muito melhor, com destaque para os testes baseados em $R^{*}$ (Barndorff-Nielsen), $R_{0}^{*}$ (DiCiccio-Martin) e $\widetilde{R}^{*}$ (Fraser-Reid-Wu) cujas discrepâncias estão próximas de zero, mesmo para níveis nominais $0,01 \%$ e $0,1 \%$ e $n=15$. As estatísticas $\bar{R}^{*}$ (Skovgaard) e $\widehat{R}^{*}$ (Severini) têm a discrepância negativa de valor-p e significativamente inferior em valor absoluto ao teste usual, com destaque para $\bar{R}^{*}$ (Skovgaard). Quando $n=30$ estes últimos apresentam discrepâncias bem próximas de zero.
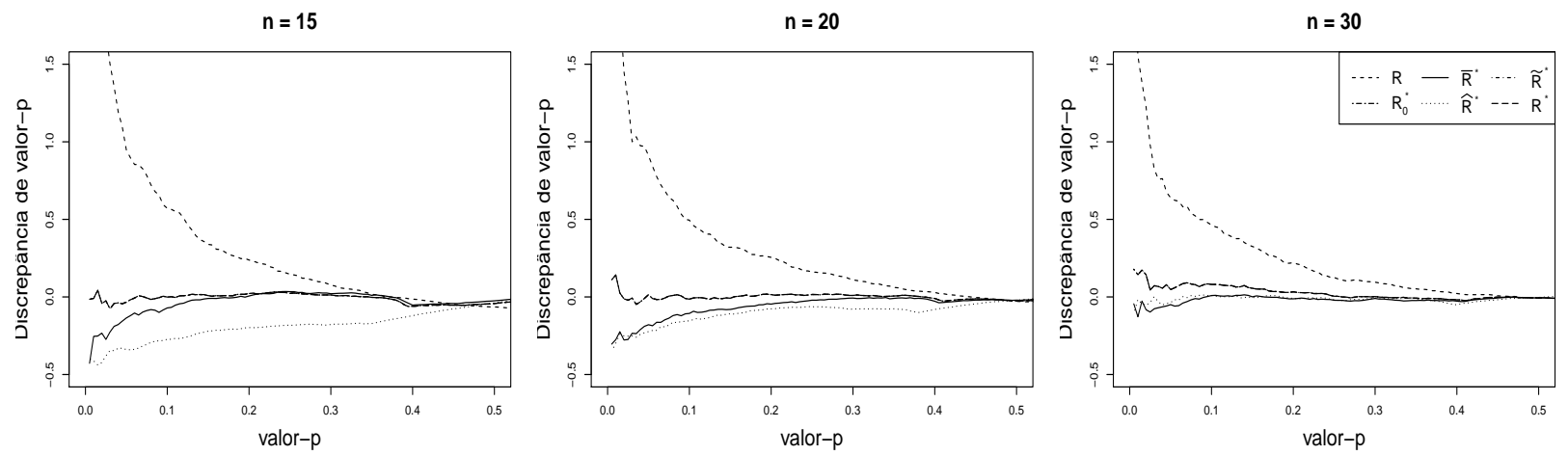

Figura 3.1: Discrepâncias relativas de valor-p, modelo 1.

Para comparação de poder, as taxas de rejeição foram obtidas sob a hipótese alternativa $\beta_{2}=\epsilon$ para diferentes valores de $\epsilon>0$ com base em 10.000 amostras de Monte Carlo. A Tabela 3.2 apresenta taxas de rejeição não nula (poder) dos testes ajustados para o modelo 1 com $n=20$ e $\alpha=10 \%$. Não há evidência de que um dos testes seja mais poderoso do que os outros. 
Tabela 3.2: Taxas de rejeição não nula (\%); modelo $1, n=20, \alpha=10 \%$

\begin{tabular}{c|ccccc}
\hline \hline$\epsilon$ & 0,1 & 0,5 & 1,0 & 2,0 & 3,0 \\
\hline$R$ & 11,7 & 22,3 & 39,8 & 76,5 & 94,6 \\
$R_{0}^{*}$ & 11,7 & 22,2 & 39,8 & 76,9 & 94,8 \\
$\bar{R}^{*}$ & 11,7 & 22,1 & 39,8 & 76,8 & 94,8 \\
$\widehat{R}^{*}$ & 11,7 & 21,6 & 37,9 & 72,5 & 91,8 \\
$\widetilde{R}^{*}$ & 11,7 & 22,2 & 39,8 & 76,9 & 94,8 \\
$R^{*}$ & 11,7 & 22,2 & 39,8 & 76,9 & 94,8 \\
\hline \hline
\end{tabular}

\subsubsection{Modelo de regressão valor extremo máximo linear com dispersão variável}

Considera-se o modelo (2.1) com os componentes sistemáticos para a localização e para a dispersão dados por

$$
\mu_{t}=\beta_{1}+\beta_{2} x_{t 2}+\beta_{3} x_{t 3}+\beta_{4} x_{t 4}
$$

$\mathrm{e}$

$$
\ln \left(\sigma_{t}\right)=\gamma_{1}+\gamma_{2} z_{t 2}+\gamma_{3} z_{t 3}
$$

o qual denominamos de 'modelo 2'. Considera-se a hipótese nula $\mathcal{H}_{0}: \beta_{4} \leq 0$ a ser testada contra a hipótese alternativa $\mathcal{H}_{1}: \beta_{4}>0$. Assume-se que $\beta_{1}=1, \beta_{2}=1, \beta_{3}=6, \beta_{4}=0, \gamma_{1}=1, \gamma_{2}=0,1$ e $\gamma_{3}=0,1$. Os valores das covariadas foram obtidos aleatoriamente de uma distribuição $\mathcal{U}(-0,5,0,5)$. Os tamanhos das amostras utilizados são 40, 50, 60 e 70.

Observa-se na Tabela 3.3 que o teste da razão de verossimilhanças sinalizada apresenta taxa de rejeição maior que os relativos níveis nominais enquanto os testes ajustados têm melhor desempenho, com as taxas de rejeição próximas dos respectivos níveis nominais. Por exemplo, para $n=40$ e $\alpha=10 \%$, a taxa de rejeição nula do teste da razão de verossimilhanças sinalizada é $12,0 \%$, enquanto a dos testes ajustados $R_{0}^{*}$ (DiCiccio-Martin) e $\bar{R}^{*}$ (Skovgaard) são 10,8\% e 10,3\%, respectivamente. Os testes ajustados $\widehat{R}^{*}$ (Severini) e $\widetilde{R}^{*}$ (Fraser-Reid-Wu) têm as taxas de rejeição próximas do nível nominal, sendo conservadores neste caso, com as taxas $9,1 \%$ e $9,5 \%$, respectivamente. Para $\alpha=5 \%$, as taxas de rejeição nula dos testes $\bar{R}^{*}$ (Skovgaard) e $\widetilde{R}^{*}$ (Fraser-Reid-Wu) estão muito próximas do nível nominal, mesmo para $n=40$, sendo $5,5 \%$ e $4,9 \%$, respectivamente. Como esperado as taxas de rejeição se aproximam do nível nominal quando o tamanho da amostra $n$ aumenta.

Tabela 3.3: Taxas de rejeição nula (\%); modelo 2, localização

\begin{tabular}{c|ccccc|ccccc|ccccc}
\hline \hline & \multicolumn{1}{|c|}{$\alpha=10 \%$} & \multicolumn{6}{c|}{$\alpha=5 \%$} & \multicolumn{5}{c}{$\alpha=1 \%$} \\
\cline { 2 - 17 }$n$ & $R$ & $R_{0}^{*}$ & $\bar{R}^{*}$ & $\widehat{R}^{*}$ & $\widetilde{R}^{*}$ & $R$ & $R_{0}^{*}$ & $\bar{R}^{*}$ & $\widehat{R}^{*}$ & $\widetilde{R}^{*}$ & $R$ & $R_{0}^{*}$ & $\bar{R}^{*}$ & $\widehat{R}^{*}$ & $\widetilde{R}^{*}$ \\
\hline 40 & 12,0 & 10,8 & 10,2 & 9,0 & 9,5 & 6,9 & 5,8 & 5,5 & 4,3 & 4,9 & 1,9 & 1,4 & 1,3 & 0,8 & 1,0 \\
50 & 11,7 & 10,6 & 9,6 & 9,3 & 9,5 & 6,4 & 5,7 & 4,9 & 4,7 & 4,8 & 1,6 & 1,3 & 0,9 & 0,8 & 0,9 \\
60 & 11,6 & 10,9 & 10,1 & 9,5 & 9,9 & 6,3 & 5,7 & 4,9 & 4,7 & 5,0 & 1,3 & 1,1 & 0,9 & 0,7 & 0,8 \\
70 & 11,4 & 11,0 & 10,2 & 9,7 & 10,2 & 5,9 & 5,6 & 4,9 & 4,7 & 4,9 & 1,4 & 1,3 & 1,1 & 0,9 & 1,0 \\
100 & 10,7 & 10,3 & 9,7 & 9,7 & 9,7 & 5,9 & 5,6 & 5,0 & 5,1 & 5,2 & 1,2 & 1,1 & 0,9 & 0,9 & 1,0 \\
200 & 10,8 & 10,7 & 10,2 & 10,4 & 10,4 & 5,4 & 5,3 & 5,0 & 5,0 & 5,0 & 1,1 & 1,1 & 1,0 & 1,0 & 1,0 \\
\hline \hline
\end{tabular}


A Figura 3.2 mostra que, no modelo linear heteroscedástico, se mantém a tendência da estatística da razão de verossimilhanças sinalizada de apresentar altos valores de discrepância de valor-p para níveis nominais pequenos. As estatísticas ajustadas corrigem bastante a discrepância sendo que $\bar{R}^{*}$ (Skovgaard) e $\widetilde{R}^{*}$ (Fraser-Reid-Wu) têm discrepância de valor-p próxima de zero para $n=50$.
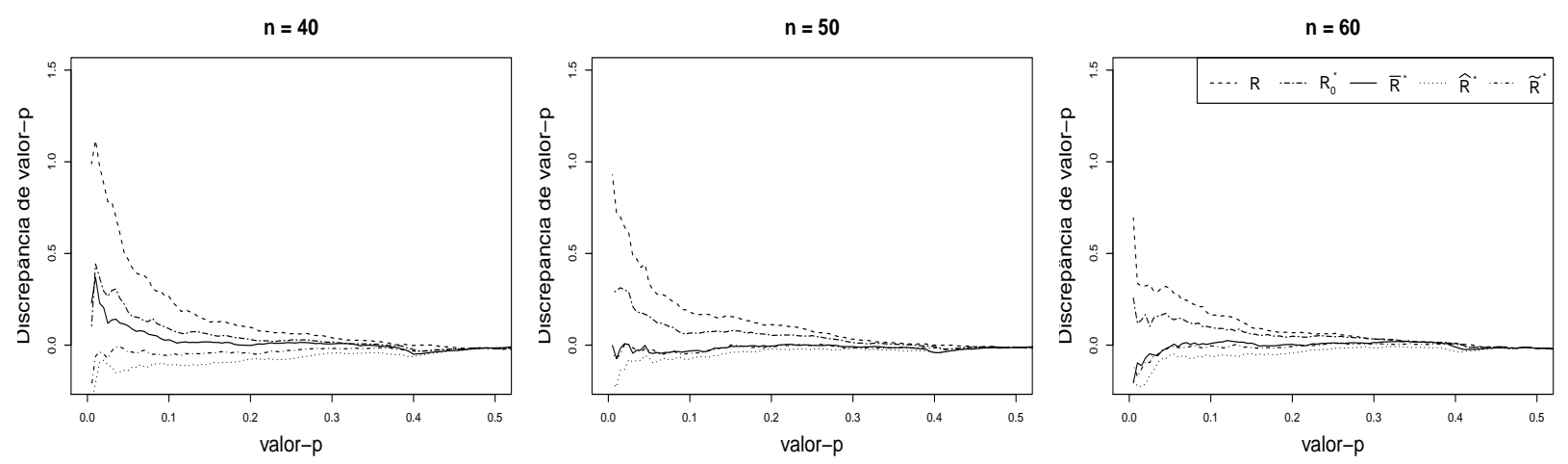

Figura 3.2: Discrepâncias relativas de valor-p; modelo 2, localização.

A Tabela 3.4 apresenta taxas de rejeição não nula (poder) dos testes para o modelo 2 com $n=40$ e $\alpha=10 \%$. Aqui, as taxas de rejeição foram obtidas sob as hipóteses alternativas $\beta_{4}=\epsilon$ para diferentes valores de $\epsilon>0$. Os poderes dos testes são similares, não havendo um teste que tenha melhor desempenho em todas as situações.

Tabela 3.4: Taxas de rejeição não nula (\%); modelo 2, localização, $n=40, \alpha=10 \%$

\begin{tabular}{c|cccccc}
\hline \hline$\epsilon$ & 0,1 & 0,5 & 1,0 & 2,0 & 4,0 & 6,0 \\
\hline$R$ & 10,4 & 14,4 & 21,5 & 39,9 & 77,6 & 95,2 \\
$R_{0}^{*}$ & 10,3 & 14,7 & 21,9 & 40,5 & 78,2 & 95,8 \\
$\widetilde{R}^{*}$ & 10,3 & 14,6 & 21,7 & 40,0 & 78,3 & 95,8 \\
$\widehat{R}^{*}$ & 10,4 & 14,4 & 21,2 & 39,2 & 76,2 & 94,5 \\
$\widetilde{R}^{*}$ & 10,4 & 14,7 & 21,6 & 40,3 & 78,3 & 95,6 \\
\hline \hline
\end{tabular}

\subsubsection{Modelo de regressão valor extremo não linear com dispersão constante}

Considera-se o modelo (2.1) com parâmetro de dispersão constante e com componente sistemático da localização dado por

$$
\mu_{t}=\beta_{0}+\beta_{1} x_{t 1}+\exp \left(\beta_{2} x_{t 2}\right)
$$

o qual denominamos de 'modelo 3'.

Considera-se a hipótese nula $\mathcal{H}_{0}: \beta_{2} \leq 0(r=1)$ a ser testada contra uma alternativa unilateral. Assume-se $\sigma=1, \beta_{0}=1, \beta_{1}=1$ e $\beta_{2}=0$. Os valores das covariadas foram obtidos aleatoriamente de uma distribuição $\mathcal{U}(0,1)$. Os tamanhos das amostras utilizados são 15, 2030 e 40.

A Tabela 3.5 mostra que no 'modelo 3' o comportamento dos testes é similar ao comportamento para o caso linear homoscedástico. O teste que usa $R$ é tipicamente liberal enquanto a taxa de rejeição dos 
outros testes estão mais próximas dos níveis nominais com destaque para os testes que usam a estatística $\widehat{R}^{*}$ (Severini) e $\widetilde{R}^{*}$ (Fraser-Reid-Wu). Por exemplo, para $n=15$ e $\alpha=10 \%$, a taxa de rejeição nula do teste que utiliza $R$ é $14,2 \%$, enquanto a dos testes baseados nas estatísticas $\widehat{R}^{*}$ (Severini) e $\widetilde{R}^{*}$ (Fraser-Reid-Wu) são $10,6 \%$ e 10,8\%, respectivamente. Neste caso, o teste ajustado $\bar{R}^{*}$ (Skovgaard) é conservador, mas a taxa de rejeição nula está próxima do nível nominal em relação ao teste da razão de verossimilhanças sinalizada, mesmo para a amostra de tamanho $n=15$. O teste ajustado $R_{0}^{*}$ (DiCiccio-Martin) é liberal mas bem menos que o teste usual. Para $n=20$ as taxas de rejeição estão bastante próximas dos níveis nominais $10 \%$, 5\% e $1 \%$, sendo, respectivamente, $10,7 \%, 5,3 \%$ e $1,3 \%$. Neste caso os testes $\widehat{R}^{*}$ (Severini) e $\widetilde{R}^{*}$ (Fraser-Reid-Wu) são os que se comportam melhor em relação ao erro do tipo I. Para $n \geq 20$ o teste $R_{0}^{*}$ (DiCiccio-Martin) também é competitivo.

Tabela 3.5: Taxas de rejeição nula (\%); modelo 3

\begin{tabular}{c|ccccc|ccccc|ccccc}
\hline \hline & \multicolumn{1}{|c|}{$\alpha=10 \%$} & \multicolumn{4}{c|}{$\alpha=5 \%$} & \multicolumn{5}{c}{$\alpha=1 \%$} \\
\cline { 2 - 16 }$n$ & $R$ & $R_{0}^{*}$ & $\bar{R}^{*}$ & $\widehat{R}^{*}$ & $\widetilde{R}^{*}$ & $R$ & $R_{0}^{*}$ & $\bar{R}^{*}$ & $\widehat{R}^{*}$ & $\widetilde{R}^{*}$ & $R$ & $R_{0}^{*}$ & $\bar{R}^{*}$ & $\widehat{R}^{*}$ & $\widetilde{R}^{*}$ \\
\hline 15 & 14,2 & 12,0 & 8,7 & 10,6 & 10,8 & 8,1 & 6,6 & 3,9 & 5,1 & 5,5 & 2,2 & 1,7 & 0,7 & 1,1 & 1,1 \\
20 & 13,3 & 10,7 & 9,0 & 10,3 & 10,5 & 7,2 & 5,3 & 4,3 & 5,0 & 5,1 & 2,1 & 1,3 & 0,9 & 1,2 & 1,2 \\
30 & 12,1 & 10,4 & 9,4 & 10,5 & 10,4 & 6,8 & 5,3 & 4,6 & 5,3 & 5,2 & 1,8 & 1,2 & 1,0 & 1,2 & 1,2 \\
40 & 11,7 & 10,3 & 9,7 & 10,7 & 10,2 & 6,5 & 5,3 & 4,7 & 5,5 & 5,2 & 1,6 & 1,1 & 1,0 & 1,2 & 1,1 \\
100 & 10,5 & 10,0 & 9,7 & 10,0 & 10,0 & 5,9 & 5,4 & 5,1 & 5,4 & 5,4 & 1,2 & 1,0 & 0,9 & 1,1 & 1,0 \\
\hline \hline
\end{tabular}

A Figura 3.3 mostra que no modelo não linear homoscedástico a estatística da razão de verossimilhanças sinalizada também exibe os maiores valores de discrepância relativa de valores-p. Para $n=15$ os testes baseados em $\widehat{R}^{*}$ (Severini) e $\widetilde{R}^{*}$ (Fraser-Reid-Wu) são os que apresentam as menores discrepâncias. Para os outros valores de $n$ apresentados, os testes se comportam de forma similar, sendo que $\bar{R}^{*}$ (Skovgaard) apresenta discrepância negativa e os demais positiva.
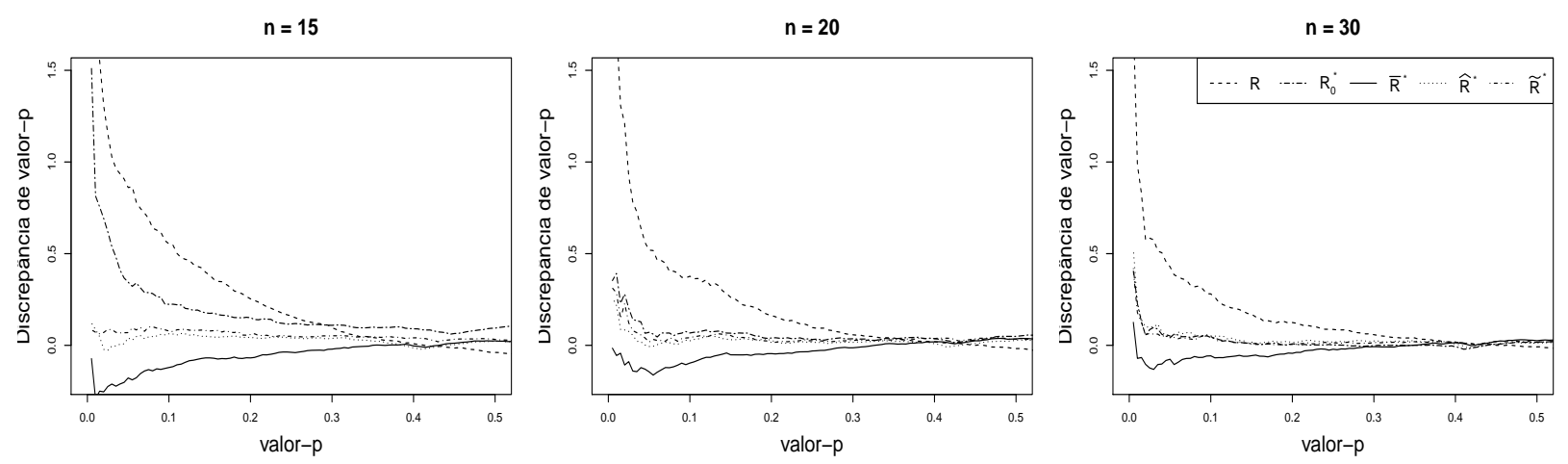

Figura 3.3: Discrepâncias relativas de valor-p; modelo 3.

A Tabela 3.6 apresenta taxas de rejeição não nula (poder) dos testes para o modelo 3 com $n=20$ e $\alpha=10 \%$. Aqui, as taxas de rejeição foram obtidas sob a hipótese alternativa $\beta_{2}=\epsilon(r=1)$, para diferentes valores de $\epsilon>0$. Os resultados obtidos para o modelo 3, mostrados na Tabela 3.6, não revelam distinção significativa dos testes em termos de poder. As conclusões obtidas para o modelo 3 não se alteram em relação às conclusões para os modelos 1 e 2. 
Tabela 3.6: Taxas de rejeição não nula (\%); modelo 3, localização, $n=15, \alpha=10 \%$

\begin{tabular}{c|cccccc}
\hline \hline$\epsilon$ & 0,01 & 0,03 & 0,05 & 0,10 & 0,20 & 0,30 \\
\hline$R$ & 12,0 & 16,2 & 21,1 & 37,5 & 76,5 & 96,3 \\
$R_{0}^{*}$ & 12,0 & 16,3 & 20,9 & 37,3 & 76,6 & 96,4 \\
$\bar{R}^{*}$ & 12,0 & 16,0 & 20,7 & 37,1 & 76,1 & 96,5 \\
$\widehat{R}^{*}$ & 11,8 & 16,3 & 21,0 & 36,8 & 75,5 & 95,6 \\
$\widetilde{R}^{*}$ & 12,0 & 16,2 & 20,9 & 37,3 & 76,5 & 96,3 \\
\hline \hline
\end{tabular}

\subsection{Aplicações}

Aplicamos os resultados obtidos neste capítulo ao conjunto de dados, chamado aqui de "decatlo" (Hand, 1996, p. 304), apresentado na Seção 2.5.

Assumimos que o resultado em salto em altura segue uma distribuição valor extremo máximo (2.1) e consideramos o modelo de regressão com dispersão constante e componente sistemático da locação dado por

$$
\mu_{t}=\beta_{0}+\beta_{1} x_{t 1}+\beta_{2} x_{t 2}+\beta_{3} x_{t 3}+\beta_{4} x_{t 4}+\beta_{5} x_{t 5}
$$

para $t=1, \ldots, 34$. As covariadas são os resultados nas seguintes provas: lançamento de dardo $\left(x_{1}\right)$, salto em distância $\left(x_{2}\right)$, lançamento de disco $\left(x_{3}\right)$, arremesso de peso $\left(x_{4}\right)$ e salto com vara $\left(x_{5}\right)$. Os resultados dos testes de hipótese são apresentados na Tabela 3.7.

Tabela 3.7: Resultado dos testes de hipótese - decatlo

\begin{tabular}{l|c|cccccc}
\hline \hline & & $R$ & $R_{0}^{*}$ & $\bar{R}^{*}$ & $\widehat{R}^{*}$ & $\widetilde{R}^{*}$ & $R^{*}$ \\
\hline $\mathcal{H}_{0}: \beta_{1} \leq 0$ contra $\mathcal{H}_{1}: \beta_{1}>0$ & estatística & 2,0102 & 1,5841 & 1,5658 & 1,5920 & 1,5945 & 1,5945 \\
& valor-p & 0,0222 & 0,0566 & 0,0587 & 0,0557 & 0,0554 & 0,0554 \\
\hline $\mathcal{H}_{0}: \beta_{4} \geq 0$ contra $\mathcal{H}_{1}: \beta_{4}<0$ & estatística & $-1,4815$ & $-1,1743$ & $-1,1277$ & $-0,5062$ & $-1,1789$ & $-1,1789$ \\
& valor-p & 0,0692 & 0,1201 & 0,1297 & 0,3064 & 0,1192 & 0,1192 \\
\hline \hline
\end{tabular}

Ao nível de significância de 5\%, apenas o teste da razão de verossimilhanças sinalizada $(R)$ rejeita a hipótese nula $\mathcal{H}_{0}: \beta_{1} \leq 0$ e os testes que usam os ajustes de Barndorff-Nielsen (1986) $\left(R^{*}\right)$, de DiCiccio \& Martin (1993) $\left(R_{0}^{*}\right)$, Skovgaard (1996) $\left(\bar{R}^{*}\right)$, Severini (1999) $\left(\widehat{R}^{*}\right)$ e Fraser et al. (1999) $\left(\widetilde{R}^{*}\right)$ não rejeitam. Ao nível de significância de $10 \%$, apenas o teste da razão de verossimilhanças sinalizada $(R)$ rejeita a hipótese nula $\mathcal{H}_{0}: \beta_{4} \geq 0$.

\subsection{Conclusões}

Neste capítulo, deduzimos a estatística da razão de verossimilhanças sinalizada ajustada, proposta por Barndorff-Nielsen (1986, 1991), para o modelo de regressão valor extremo máximo linear homocedástico e suas aproximações para o caso geral. Observa-se através das simulações que o teste da razão de verossimilhanças sinalizada resulta em probabilidades de erro de tipo I acima dos níveis nominais correspondentes enquanto os testes corrigidos apresentam maior acurácia. No caso linear homoscedástico é possível obter 
o ajuste de Barndorff-Nielsen $\left(R^{*}\right)$. Neste caso os testes ajustados $R^{*}$ (Barndorff-Nielsen), $R_{0}^{*}$ (DiCiccioMartin) e $\widetilde{R}^{*}$ (Fraser-Reid-Wu) são competitivos entre si e têm melhor desempenho que os demais testes ajustados analisados. No caso linear heteroscedástico, $\bar{R}^{*}$ (Skovgaard) e $\widetilde{R}^{*}$ (Fraser-Reid-Wu) apresentam a menor discrepância em relação à assíntota, sendo liberal e conservador respectivamente, quando a amostra é pequena. No caso não linear homoscedástico contemplado, $\widehat{R}^{*}$ (Severini) e $\widetilde{R}^{*}$ (Fraser-Reid-Wu) se equiparam em acurácia. Considerando as situações estudadas, os testes apresentaram alguma variação de um caso para outro mas o teste $\widetilde{R}^{*}$ (Fraser-Reid-Wu) esteve entre os melhores nas três configurações.

A aplicação dos testes estudados a um conjunto real de dados mostra que a conclusão, para este conjunto de dados, se altera ao utilizarmos o teste da razão de verossimilhanças usual e os testes ajustados. Lembrando que o teste usual é muito liberal quando o tamanho da amostra é pequeno (nesta aplicação, $n=34$ ), sugerimos fazer inferência com base nos testes ajustados. 


\section{Capítulo 4}

\section{Generalizações da distribuição valor extremo máximo ou Gum- bel}

\subsection{Introdução}

Muitos estudos empíricos concluíram que certas situações tendem a apresentar uma distribuição de probabilidade com excesso de curtose. Por exemplo, retornos de ativos financeiros (Danielsson et al., 2006), hidrodinâmica de rios (Bruxer et al., 2008), turbulência em ecossistemas aquáticos (Sanford, 1997), ou ainda, vitórias em jogos de azar relacionadas à decisão de parar (Lien, 2001). Como aclarado em Danielsson et al. (2006), excesso de curtose de uma distribuição ocorre se a distribuição tem cauda mais pesada do que a normal, se o centro da distribuição é mais pontiaguda ou se ambas as características estão presentes.

$\mathrm{Na}$ área de modelagem climática, uma das distribuições mais utilizadas é a distribuição valor extremo máximo ou Gumbel. A Teoria do Valor Extremo foi proposta por Leonard Henry Caleb Tippet (1902-1985). Trabalhando numa indústria têxtil com a função de tornar a fibra de algodão mais forte, percebeu que a fragilidade de uma linha era influenciada pela fragilidade da fibra mais fraca. Com a ajuda de Ronald Aylmer Fisher (1890-1962), ele obteve os três limites assintóticos que descrevem as distribuições dos valores extremos, o mínimo e o máximo. Gumbel formalizou esta teoria em seu livro de 1958, Statistics of Extremes, incluindo a distribuição Gumbel, um dos três limites, que recebeu este nome em sua homenagem. Gumbel mostrou que o máximo (a última estatística de ordem) de uma amostra de uma variável aleatória com distribuição exponencial se aproxima de uma distribuição Gumbel conforme aumenta o tamanho da amostra.

No que segue a variável valor extremo máximo ou Gumbel será chamada simplesmente de Gumbel e denotada por $E V$. Seja uma variável aleatória $X$ com distribuição Gumbel, $X \sim E V(\mu, \sigma)$, a função densidade de probabilidade e função de probabilidade acumulada são dadas, respectivamente, por

$$
f_{E V}(x ; \mu, \sigma)=\frac{1}{\sigma} \exp \left(\left(-\frac{x-\mu}{\sigma}\right)-\exp \left(-\frac{x-\mu}{\sigma}\right)\right), \quad x \in \mathbb{R}
$$

e

$$
F_{E V}(x ; \mu, \sigma)=\exp \left(-\exp \left(-\frac{x-\mu}{\sigma}\right)\right), \quad x \in \mathbb{R},
$$

em que $\mu \in \mathbb{R}$ é o parâmetro de localização e $\sigma>0$ o parâmetro de dispersão. A Figura 4.1 apresenta algumas densidades Gumbel com seus valores correspondentes de $(\mu, \sigma)$. 


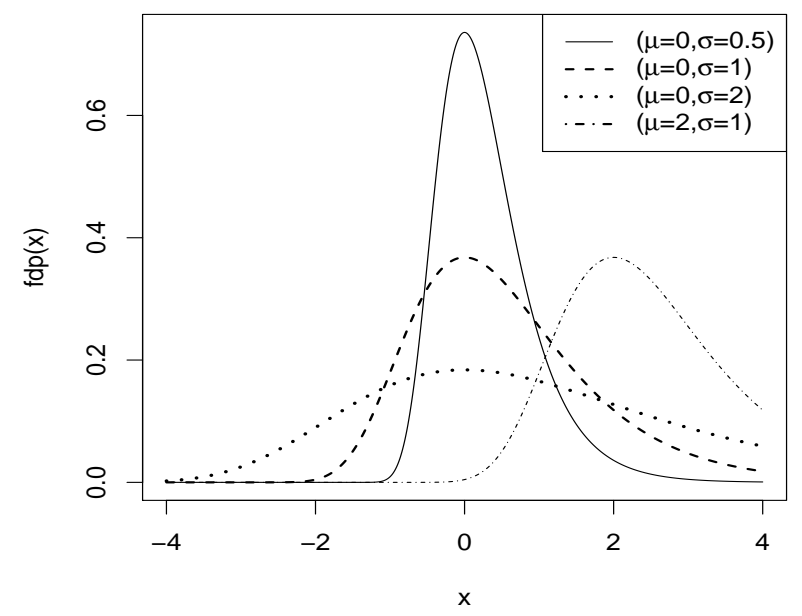

Figura 4.1: Função densidade de probabilidade de distribuição $\operatorname{Gumbel}(\mu, \sigma)$.

A média, mediana e a variância de $X$ são

$$
\begin{gathered}
\mathrm{E}(X)=\mu+\mathcal{E} \sigma, \\
m(X)=\mu-\sigma \ln \ln 2
\end{gathered}
$$

e

$$
\operatorname{var}(X)=\frac{\pi^{2}}{6} \sigma^{2}
$$

respectivamente, onde $\mathcal{E}$ é a constante de Euler; $\mathcal{E} \approx 0,5772$ (ver Apêndice F.1).

O quantil $x_{p}$, tal que $F_{E V}\left(x_{p} ; \mu, \sigma\right)=p$, é obtido invertendo-se a função de distribuição acumulada dada em (4.2), e é dado por

$$
x_{p}=\mu-\sigma \ln (-\ln (p)) .
$$

A variável aleatória $X \sim E V(\mu, \sigma)$ pode, portanto, ser gerada diretamente de uma distribuição uniforme com parâmetros 0 e 1 , digamos $U \sim \operatorname{Unif}(0,1)$, por $X=\mu-\sigma \ln (-\ln (U))$.

O momento de ordem $n$ é dado por

$$
\mathrm{E}\left(X^{n}\right)=\sum_{i=0}^{n}\left(\begin{array}{l}
n \\
i
\end{array}\right) \mu^{n-i}(-\sigma)^{i} \Gamma^{(i)}(1),
$$

em que $\Gamma(x)=\int_{0}^{\infty} t^{x-1} e^{-t} d t$ e $\operatorname{Re} x>0$ é a função gama e sua $n$-ésima derivada é $\Gamma^{(n)}(x)=$ $\int_{0}^{\infty} t^{x-1}(\ln t)^{n} e^{-t} d t$.

Os coeficientes de assimetria $\left(\gamma_{1}\right)$ e de curtose $\left(\gamma_{2}\right)$ de uma variável aleatória $X$ são definidas, respecti- 
vamente, por

$$
\gamma_{1}=\frac{E\left((X-E(X))^{3}\right)}{\left[E\left((X-E(X))^{2}\right)\right]^{3 / 2}}=\frac{\mathrm{E}\left(X^{3}\right)-3 \mathrm{E}(X) \mathrm{E}\left(X^{2}\right)+2 \mathrm{E}(X)^{3}}{\left(\mathrm{E}\left(X^{2}\right)-\mathrm{E}(X)^{2}\right)^{3 / 2}}
$$

$\mathrm{e}$

$$
\gamma_{2}=\frac{E\left((X-E(X))^{4}\right)}{\left[E\left((X-E(X))^{2}\right)\right]^{2}}=\frac{\mathrm{E}\left(X^{4}\right)-4 \mathrm{E}(X) \mathrm{E}\left(X^{3}\right)+6 \mathrm{E}(X)^{2} \mathrm{E}\left(X^{2}\right)+3 \mathrm{E}(X)^{4}}{\left(\mathrm{E}\left(X^{2}\right)-\mathrm{E}(X)^{2}\right)^{2}}
$$

Se $Z$ é uma variável aleatória, $\mu \in \mathbb{R}$ e $\sigma>0$, temos que os coeficientes de assimetria e de curtose de $\mu+\sigma Z$ são dados por

$$
\mathrm{E}\left([(\mu+\sigma Z)-\mathrm{E}(\mu+\sigma Z)]^{k}\right)=\sigma^{k} \mathrm{E}\left([(Z-\mathrm{E}(Z))]^{k}\right)
$$

e, portanto,

$$
\gamma_{1}(\mu+\sigma Z)=\gamma_{1}(Z) \quad \text { e } \quad \gamma_{2}(\mu+\sigma Z)=\gamma_{2}(Z)
$$

Os coeficientes de assimetria e de curtose da distribuição Gumbel não dependem de parâmetros, são constantes iguais a $\gamma_{1 ; E V}=12 \sqrt{6} \zeta(3) / \pi^{3} \cong 1,139547$ e $\gamma_{2 ; E V}=5,4$, respectivamente, em que $\zeta(s)=$ $\sum_{k=1}^{\infty} k^{-s}, \operatorname{Re} s>1$ é a função zeta de Riemann e $\zeta(3) \approx 1,2$. O fato dos coeficientes de assimetria e curtose da distribuição Gumbel serem constantes motiva generalizações visando obter distribuições mais flexíveis e úteis para modelar dados reais (ver Apêndice F.1).

Neste capítulo apresentamos o resultado de uma revisão da literatura feita com o objetivo de listar as distribuições que têm como caso particular ou limite a distribuição Gumbel. Relacionamos, para cada uma das distribuições encontradas, seus momentos, quantis, procedimento de geração de observações, seus coeficientes de assimetria e de curtose e apontamos quais famílias de distribuições são não identificáveis ${ }^{1}$. Segundo Huang (2005), “ when applying a nonidentifiable model, different people may draw different conclusions from the same model of the observed data. Before one can meaninfully discuss the estimation of a model, model identifiability must be verified". Apresentamos os conceitos de funcão de variação regular e índice da cauda de uma distribuição e classificamos as distribuições contempladas que são identificáveis. Apresentamos uma descrição de medidas de qualidade de ajuste que serão usadas nas aplicações. Ajustamos as distribuições identificáveis a um conjunto de dados simulados e a um conjunto real de dados de velocidade de vento. Fazendo uso de várias medidas de qualidade de ajuste comparamos tais distribuições em sua capacidade de modelar dados de uma distribuição Gumbel e em relação a flexibilidade em acomodar dados com presença de pontos muito discrepantes.

\subsection{Generalizações da distribuição valor extremo máximo ou Gumbel}

Apresentamos, a seguir, algumas distribuições que têm como caso particular ou limite a distribuição Gumbel.

\subsubsection{Distribuição valor extremo generalizada}

Dentre muitos estudiosos que contribuíram para a teoria do valor extremo podemos destacar cronologicamente alguns nomes. Bernoulli em 1790 discutiu sobre a média da distância máxima da origem de $n$

\footnotetext{
${ }^{1}$ Uma família de distribuições com função densidade de probabilidade $f(x ; \theta), \theta \in \Theta$, é dita identificável se, para $\theta$ e $\theta^{*}$ pertencentes ao espaço paramétrico $\Theta, f(x ; \theta)=f\left(x ; \theta^{*}\right) \Leftrightarrow \theta=\theta^{*}$.
} 
pontos distribuídos aleatoriamente sobre uma reta de tamanho fixo $t$ (ver Gumbel, 1958). Fréchet (1927) apresentou uma possível distribuição limite para o máximo. Fisher \& Tippet (1928) mostraram que o limite da distribuição de máximos só pode ser um entre três tipos. Von Mises (1936) apresentou algumas condições suficientes para a convergência fraca do máximo para cada uma das três distribuições limites possíveis. Gumbel (1937a,b) tratou com aplicações práticas da estatística de valor extremo nas áreas de tempo de vida de seres humanos e emissões radioativas. Weibull (1939) estudou a resistência dos materiais e fez uma aplicação prática da distribuição do valor extremo mínimo. Gumbel (1941, 1944, 1945, 1948) fez aplicações na área meteorológica (enchentes anuais, precipitação máxima, etc). Gnedenko (1943) apresentou uma fundamentação teórica rigorosa da teoria do valor extremo e definiu condições necessárias e suficientes para a convergência fraca das estatísticas de ordem extremas. A distribuição valor extremo generalizada (GEV) foi definida pela primeira vez por Jenkinson (1955) e é uma forma única de apresentar os três possíveis limites da distribuição do valor extremo máximo. A distribuição também é referida como distribuição valor extremo tipo von Mises, distribuiçao von Mises-Jenkinson e ainda distribuição Fisher-Tippet. Outras contribuições importantes e aplicações são relacionadas em Kotz \& Nadajarah (2000).

Seja $X$ uma variável aleatória com distribuição valor extremo generalizada, digamos $X \sim$ $G E V(\mu, \sigma, \alpha)$. A função densidade de probabilidade e a função de distribuição acumulada são dadas, respectivamente, por

$$
\begin{aligned}
& f_{G E V}(x ; \mu, \sigma, \alpha)=\frac{1}{\sigma} \exp \left(-\left[1+\alpha\left(\frac{x-\mu}{\sigma}\right)\right]^{-1 / \alpha}\right)\left[1+\alpha\left(\frac{x-\mu}{\sigma}\right)\right]^{-1 / \alpha-1} \\
& \{x: 1+\alpha(x-\mu) / \sigma>0\}
\end{aligned}
$$

e

$$
F_{G E V}(x ; \mu, \sigma, \alpha)=\exp \left(-\left[1+\alpha\left(\frac{x-\mu}{\sigma}\right)\right]^{-1 / \alpha}\right), \quad\{x: 1+\alpha(x-\mu) / \sigma>0\},
$$

em que $\mu \in \mathbb{R}, \sigma>0$ e $\alpha \in \mathbb{R}$. A distribuição do valor extremo generalizada (GEV) tem como caso particular a distribuição do Gumbel quando $\alpha \rightarrow 0$. A Figura 4.2 apresenta a função densidade de probabilidade para $\operatorname{GEV}(0,1, \alpha)$ para alguns valores de $\alpha$. Pode-se observar que o parâmetro $\alpha$ influencia a localização, dispersão, assimetria e curtose e que o aumento do módulo de $\alpha$ corresponde ao aumento do valor da moda.

A esperança, mediana e variância são dadas, respectivamente, por

$$
\begin{gathered}
\mathrm{E}(X)= \begin{cases}\mu+\sigma(\Gamma(1-\alpha)-1) / \alpha, & \text { se } \alpha \neq 0 \text { e se } 1-\alpha>0 ; \\
\mu+\sigma \mathcal{E}, & \text { se } \alpha=0 ;\end{cases} \\
m= \begin{cases}\mu+\sigma\left(\ln (2)^{-\alpha}-1\right) / \alpha, & \text { se } \alpha \neq 0 \\
\mu-\sigma \ln \ln 2, & \text { se } \alpha=0 ;\end{cases}
\end{gathered}
$$

e

$$
\operatorname{var}(X)= \begin{cases}\left(\frac{\sigma}{\alpha}\right)^{2}\left(\Gamma(1-2 \alpha)-\Gamma^{2}(1-\alpha)\right), & \text { se } \alpha \neq 0 \text { e se } 1-2 \alpha>0 \\ \sigma^{2} \pi^{2} / 6, & \text { se } \alpha=0\end{cases}
$$




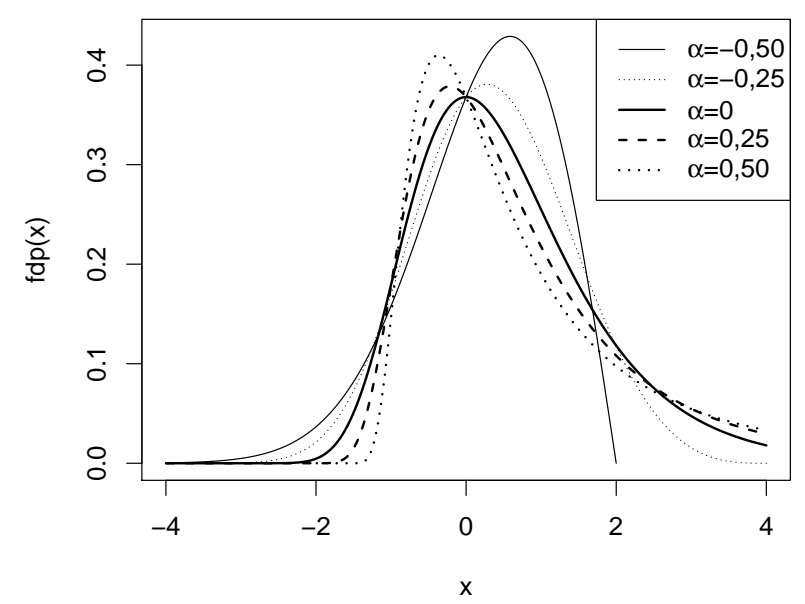

Figura 4.2: Função densidade de probabilidade de distribuição valor extremo generalizada $\operatorname{GEV}(0,1, \alpha)$.

(ver Apêndice F.1).

O quantil $x_{p}$ é dado por

$$
x_{p}= \begin{cases}\mu+(\sigma / \alpha)\left((-\ln (p))^{-\alpha}-1\right), & \text { se } \alpha \neq 0, \\ \mu-\sigma \ln (-\ln (p)), & \text { se } \alpha=0 .\end{cases}
$$

A variável aleatória $X \sim G E V(\mu, \sigma, \alpha)$ pode, portanto, ser gerada diretamente de $U \sim U n i f(0,1)$ por

$$
X= \begin{cases}\mu+(\sigma / \alpha)\left((-\ln (U))^{-\alpha}-1\right), & \text { se } \alpha \neq 0 \\ \mu-\sigma \ln (-\ln (U)), & \text { se } \alpha=0\end{cases}
$$

A distribuição GEV é flexível em relação a assimetria e curtose mas, para certos valores do parâmetro $\alpha$, os coeficientes de assimetria e de curtose não estão definidos. Estes são dados, respectivamente, por

$$
\gamma_{1, \mathrm{GEV}}= \pm \frac{\Gamma(1-3 \alpha)-3 \Gamma(1-2 \alpha) \Gamma(1-\alpha)+2 \Gamma^{3}(1-\alpha)}{\left(\Gamma(1-2 \alpha)-\Gamma^{2}(1-\alpha)\right)^{3 / 2}}
$$

sendo o sinal positivo quando $\alpha>0$ e negativo caso contrário, e

$$
\gamma_{2, \mathrm{GEV}}=\frac{\Gamma(1-4 \alpha)-4 \Gamma(1-3 \alpha) \Gamma(1-\alpha)+6 \Gamma(1-2 \alpha) \Gamma^{2}(1-\alpha)-3 \Gamma^{4}(1-\alpha)}{\left(\Gamma(1-2 \alpha)-\Gamma^{2}(1-\alpha)\right)^{2}}
$$

(ver Apêndice F.1) . O coeficiente de assimetria está bem definido para $\alpha<1 / 3$ e o de curtose para $\alpha<1 / 4$, caso em que todos os valores em que a função $\Gamma$ é calculada são maiores que zero . A Figura 4.3 apresenta os gráficos dos coeficientes de assimetria e de curtose da distribuição valor extremo generalizada e podemos notar que esta é bem mais flexível que a distribuição Gumbel. A distribuição do valor extremo generalizada pode modelar uma ampla gama de valores de assimetria e inclui distribuições menos e mais leptocúrticas do 
que a Gumbel $\left(\gamma_{2, E V}=5,4\right)$.
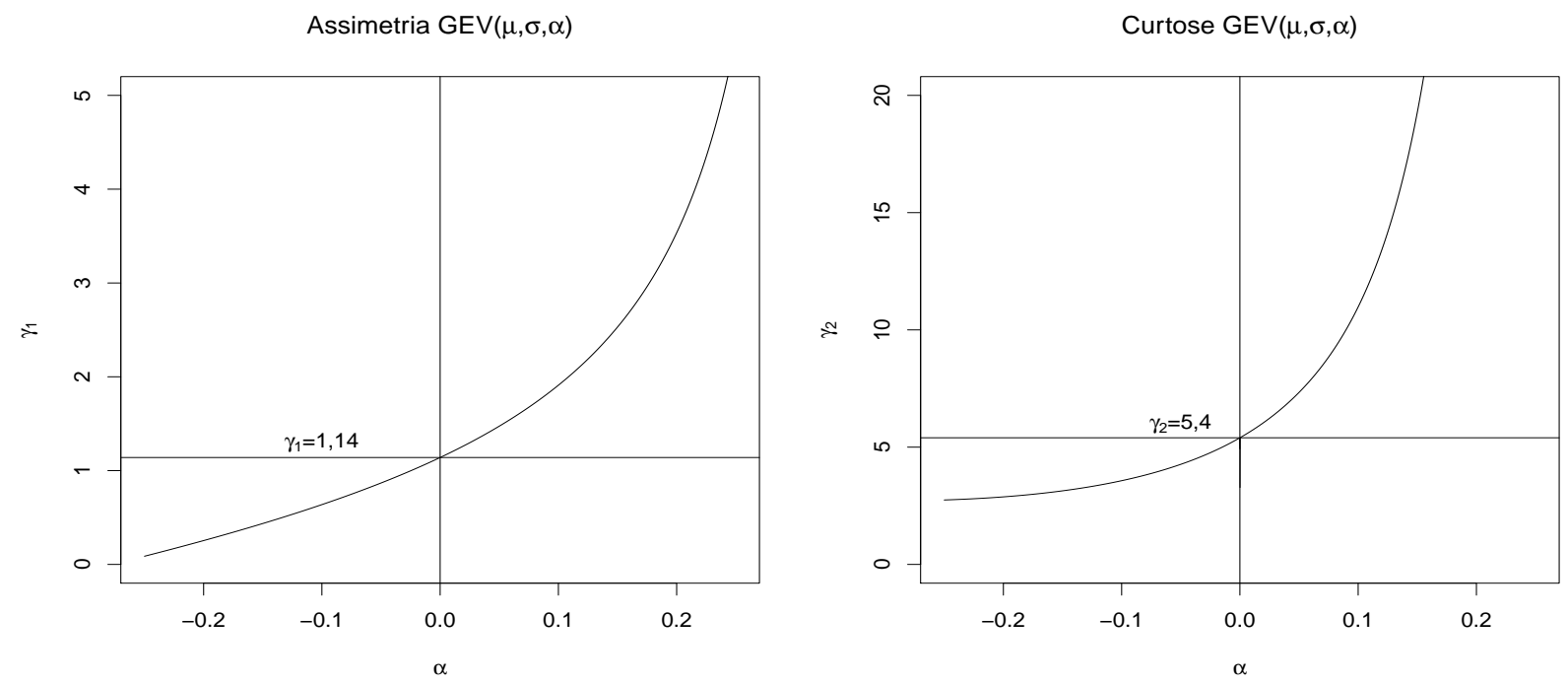

Figura 4.3: Gráficos dos coeficientes de assimetria e de curtose da distribuição valor extremo generalizada $G E V(\mu, \sigma, \alpha)$ em função de $\alpha$.

A distribuição valor extremo generalizada não satisfaz condições de regularidade usuais uma vez que seu suporte depende dos parâmetros pois está definida para $\{z: 1+\alpha(z-\mu) / \sigma>0\}$, em que $\mu \in \mathbb{R}$, $\sigma>0$ e $\alpha \in \mathbb{R}$. Quando $\alpha<0$ ou $\alpha>0, \mu-\sigma / \alpha$ é, respectivamente, um limite superior e um limite inferior no suporte da distribuição. Esta violação das condições de regularidade implica que os resultados assintóticos da verossimilhança não são sempre válidos. Segundo Coles (2001, p. 55), quando $\alpha<-1$ é improvável obter o estimador de máxima verossimilhança, quando $-1<\alpha<-0,5$ é geralmente obtido mas não tem as propriedades assintóticas padrão e quando $\alpha>-0,5$ os estimadores de máxima verossimilhança são regulares no sentido de ter as propriedades assintóticas usuais. O caso em que $\alpha \leq-0,5$ corresponde a distribuições com caudas superiores muito curtas e que tais situações são improváveis de serem encontradas em aplicações de modelos de valores extremos, o que não inviabiliza o uso da distribuição na prática. Kotz \& Nadajarah (2000, p. 62) acrescentam ainda que para $\alpha>0,5$ o segundo momento e de ordens superiores não existem. Dados reais sugerem que a condição $-0,5<\alpha<0,5$ é quase sempre satisfeita em aplicações práticas, em particular em dados ambientais.

\subsubsection{Distribuição Gumbel exponencializada}

Uma outra generalização da distribuição Gumbel foi proposta em Nadarajah (2006) inspirada na distribuição exponencial exponencializada e por isso denominada de Gumbel exponencializada.

Seja $X$ uma variável aleatória com distribuição Gumbel exponencializada, $X \sim E G u(\mu, \sigma, \alpha)$. A função densidade de probabilidade e a função de distribuição acumulada são definidas, respectivamente, por

$f_{E G u}(x ; \mu, \sigma, \alpha)=\frac{\alpha}{\sigma} \exp \left(-\frac{x-\mu}{\sigma}\right) \exp \left(-\exp \left(-\frac{x-\mu}{\sigma}\right)\right)\left[1-\exp \left(-\exp \left(-\frac{x-\mu}{\sigma}\right)\right)\right]^{\alpha-1}$, $x \in \mathbb{R}$, 
$\mathrm{e}$

$$
F_{E G u}(x ; \mu, \sigma, \alpha)=1-\left[1-\exp \left(-\exp \left(-\frac{x-\mu}{\sigma}\right)\right)\right]^{\alpha}, \quad x \in \mathbb{R},
$$

em que $\mu \in \mathbb{R}$ é o parâmetro de localização, $\sigma>0$ o parâmetro de dispersão e $\alpha>0$. Quando $\alpha=$ 1 a função densidade de probabilidade da distribuição Gumbel exponencializada é a de uma distribuição Gumbel. A Figura 4.4 apresenta algumas densidades da distribuição Gumbel exponencializada com seus valores correspondentes de $\alpha$.

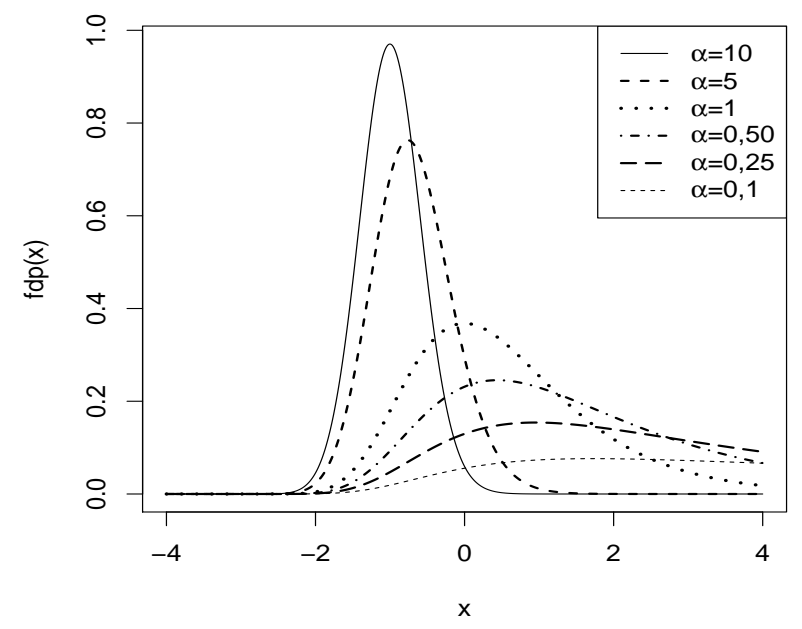

Figura 4.4: Função densidade de probabilidade da distribuição Gumbel exponencializada $E G u(0,1, \alpha)$.

A função densidade de probabilidade pode ser escrita como

$$
\begin{gathered}
f_{E G u}(x ; \mu, \sigma, \alpha)= \\
\frac{\alpha}{\sigma} \exp \left(-\frac{x-\mu}{\sigma}\right) \exp \left(-\exp \left(-\frac{x-\mu}{\sigma}\right)\right){ }_{2} F_{1}\left(1-\alpha, 1,1, \exp \left(-\exp \left(-\frac{x-\mu}{\sigma}\right)\right)\right),
\end{gathered}
$$

em que ${ }_{2} F_{1}(a, b, c, z)$ é a função Hipergeométrica, definida por

$$
{ }_{2} F_{1}(a, b, c, z)=\sum_{k=0}^{\infty} \frac{(a)_{k}(b)_{k}}{(c)_{k}} \frac{z^{k}}{k !} \quad \text { para } \quad|z|<1,
$$

e $(a)_{k}=\Gamma(a+k) / \Gamma(a)$ é o símbolo de Pochhammer. Portanto ${ }_{2} F_{1}(a, 1,1, z)=\sum_{k=0}^{\infty}(a)_{k} z^{k} / k !=$ $(1-z)^{-a}$ para $|z|<1$. Temos que $|\exp (-\exp (-(x-\mu) /(\sigma)))|<1$.

O momento de ordem $n$ é dado por

$$
\mathrm{E}\left(X^{n}\right)=\int_{0}^{1} \alpha(\mu-\sigma \ln (-\ln y))_{2}^{n} F_{1}(1-\alpha, 1,1, y) d y
$$

O n-ésimo momento pode ser obtido por integração numérica para cada valor particular do parâmetro 
$(\mu, \sigma, \alpha)$.

O quantil $x_{p}$ é dado por

$$
x_{p}=\mu-\sigma \ln \left(-\ln \left(1-(1-p)^{1 / \alpha}\right)\right) .
$$

A variável aleatória $X \sim E G u(\mu, \sigma, \alpha)$ pode, portanto, ser gerada diretamente de $U \sim \operatorname{Unif}(0,1)$ por $X=\mu-\sigma \ln \left(-\ln \left(1-(1-U)^{1 / \alpha}\right)\right)$.

Os coeficientes de assimetria $\left(\gamma_{1}\right)$ e de curtose $\left(\gamma_{2}\right)$ da distribuição Gumbel exponencializada $E G u(\mu, \sigma, \alpha)$ são obtidas através dos momentos (4.9) e das equações (4.6) e (4.7). O mínimo e o máximo da assimetria são $-0,692$ e 1,971, para $\alpha=21770,7$ e $\alpha=0.0807327$, respectivamente. O mínimo e o máximo da curtose são 3,023 e 8, 84953, para $\alpha=17,204$ e $\alpha=0,091$, respectivamente. A Figura 4.5 apresenta os gráficos dos coeficientes de assimetria e de curtose da distribuição Gumbel exponencializada $E G u(\mu, \sigma, \alpha)$ e podemos observar que é flexível em relação a Gumbel (ver Apêndice F.2).
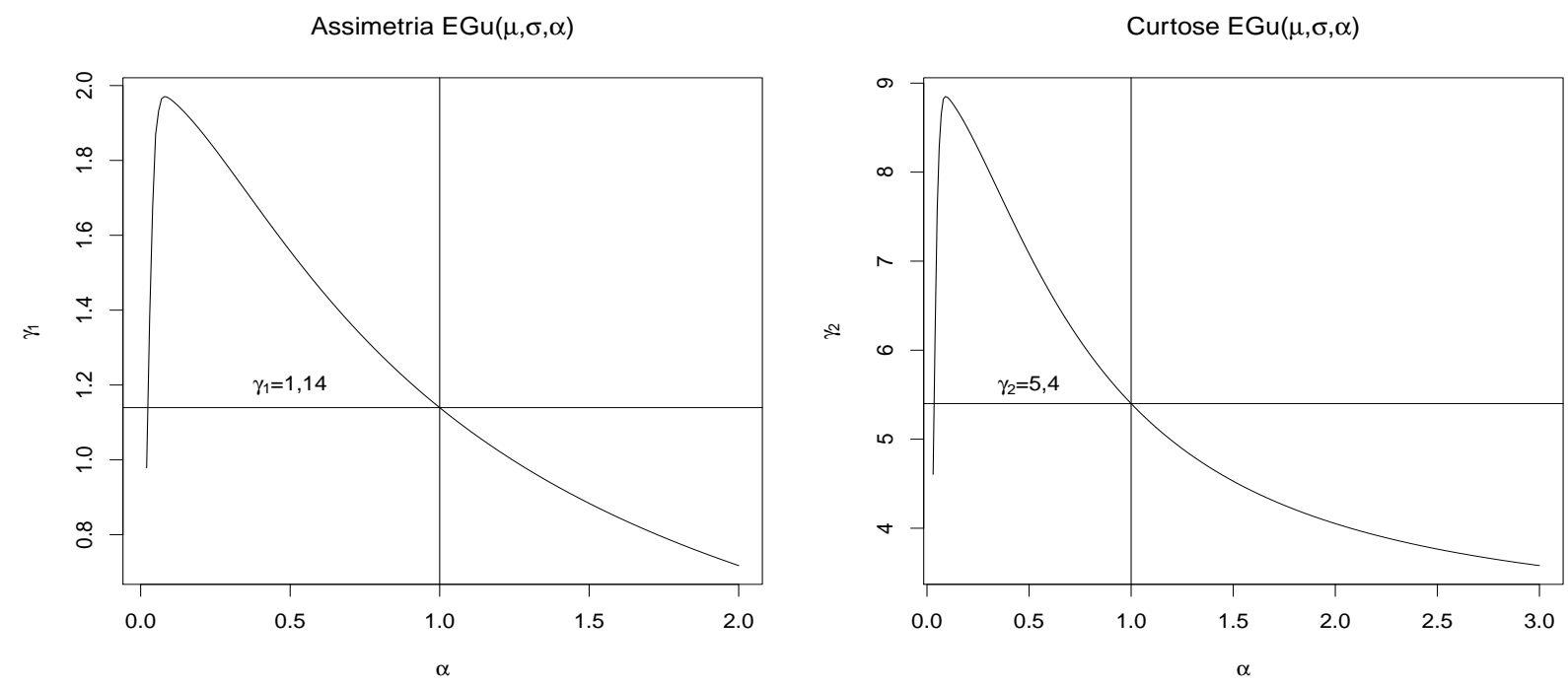

Figura 4.5: Gráficos dos coeficientes de assimetria e de curtose da distribuição Gumbel exponencializada $E G u(\mu, \sigma, \alpha)$ em função de $\alpha$.

\subsubsection{Distribuição valor extremo transmutada}

Shaw \& Buckley (2007) definiram uma transformação, a qual denominaram de transmutação de classificação (rank transmutation map), com a finalidade de obter distribuições assimétricas e com curtose distinta da normal. A transmutação é a composição de uma certa função de distribuição acumulada com a função quantílica (inversa da distribuição acumulada) de uma outra distribuição. Eles apresentaram uma transformação baseados no fato de que a distribuição de uma função de distribuição é uma uniforme no intervalo $(0,1)$ e no método de obtenção da função de densidade de uma variável transformada através da função de distribuição. Esta distribuição, denominada transmutação da amostra (sample transmutation map) $T_{S}$ é definida a partir de duas distribuições $F_{1}(x)$ e $F_{2}(x)$ tal que $T_{S}(z)=F_{2}^{-1}\left(F_{1}(z)\right)$, em que $F^{-1}=Q_{F}$ é a função quantílica de $F$, a qual transmuta uma amostra de uma distribuição $F_{1}$ em uma amostra de uma distribuição $F_{2}$. Esta transformação de transmutação é utilizada, por exemplo, em teoria assintótica 
na expansão de Cornish-Fisher. Inspirados nesta transformação, eles propuseram uma transformação correspondente, a qual denominaram transmutação de classificação (rank transmutation map) $T_{R}$. Dadas duas distribuições $F_{1}(x)$ e $F_{2}(x)$, definidas no mesmo espaço amostral, a transmutação de classificação é definida por $T_{R}(z)=F_{2}\left(F_{1}^{-1}(z)\right)$ e gera uma distribuição mais flexível em relação a assimetria e curtose.

Um caso particular, possivelmente mais simples, de transmutação de classificação é obtido considerando, para $|\alpha| \leq 1$,

$$
T_{R}(u)=F_{2}\left(F_{1}^{-1}(u)\right)=u+\alpha u(1-u)
$$

$\log 0$

$$
F_{2}(x)=(1+\alpha) F_{1}(x)-\alpha F_{1}^{2}(x),
$$

a qual é denominada de transmutação quadrática. Há dois casos extremos importantes. Quando $\alpha=-1$, $F_{2}(x)=F_{1}(x)^{2}$, isto é, $F_{2}$ corresponde à distribuição do máximo de duas variáveis independentes com distribuição $F_{1}$. Analogamente, quando $\alpha=1, F_{2}$ corresponde à distribuição do mínimo. Não há restrições sobre a distribuição de base $\left(F_{1}\right)$, mas se esta for simétrica em torno da origem, a distribuição transmutada preserva os momentos pares da função de base. Por exemplo, a distribuição do quadrado da distribuição transmutada coincide com a distribuição do quadrado da distribuição de base, independentemente do valor de $\alpha$. Isto não ocorre para transmutação cúbica, biquadrática ou de ordem superior; (Shaw \& Buckley (2007, p. 16).

Motivados pela ampla aplicação da teoria do valor extremo, especialmente a distribuição do valor extremo máximo, ou valor extremo Tipo I ou, ainda, Gumbel, Aryal \& Tsokos (2009), usaram-na como função base de uma distribuição transmutada quadrática obtendo a função de distribuição acumulada de uma variável aleatória a qual chamaremos de valor extremo transmutado (TEV) com três parâmetros, a saber, $\mu$ parâmetro de posição, $\sigma$ escala e $\alpha$ forma.

Seja $X$ uma váriável aleatória com distribuição valor extremo transmutada com parâmetros, $\mu, \sigma \mathrm{e} \alpha$, $X \sim T E V(\mu, \sigma, \alpha)$, sua função densidade de probabilidade é dada por

$$
\begin{aligned}
& f_{T E V}(x ; \mu, \sigma, \alpha)=\frac{1}{\sigma} \exp \left[-\frac{x-\mu}{\sigma}-\exp \left(-\frac{x-\mu}{\sigma}\right)\right]\left[1+\alpha-2 \alpha \exp \left(-\exp \left(-\frac{x-\mu}{\sigma}\right)\right)\right], \\
& x \in \mathbb{R}
\end{aligned}
$$

e sua função de distribuição acumulada por

$$
F_{T E V}(x ; \mu, \sigma, \alpha)=(1+\alpha) \exp \left[-\exp \left(-\frac{x-\mu}{\sigma}\right)\right]-\alpha \exp \left[-2 \exp \left(-\frac{x-\mu}{\sigma}\right)\right], \quad x \in \mathbb{R},
$$

em que $\mu \in \mathbb{R}$ é o parâmetro de localização, $\sigma>0$ o parâmetro de dispersão e $|\alpha| \leq 1$. Quando $\alpha=-1$ ou $\alpha=0$ a distribuição valor extremo transmutada coincide com a distribuição Gumbel. Note que , para tornar a família de distribuições $\operatorname{TEV}(\mu, \sigma, \alpha)$ identificável basta restringir $\alpha \in(-1,1]$. A Figura 4.6 apresenta a função densidade de probabilidade de uma distribuição $\operatorname{TEV}(0,1, \alpha)$ para alguns valores de $\alpha$.

Aryal \& Tsokos (2009) obtiveram os momentos de ordem $n$ desta distribuição, em particular, a espe- 


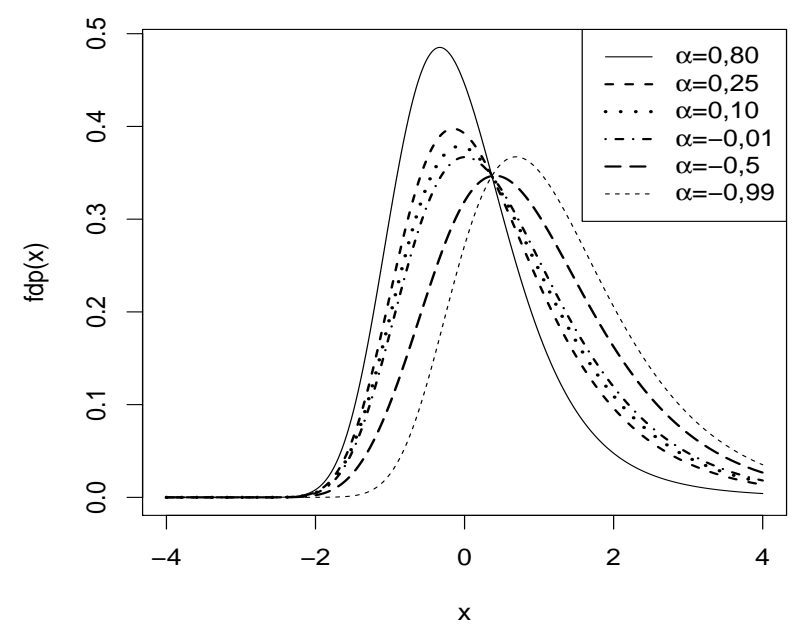

Figura 4.6: Função densidade de probabilidade da distribuição $T E V(0,1, \alpha)$.

rança e a variância, dadas por

$$
\begin{gathered}
\mathrm{E}(X)=(\mu+\mathcal{E} \sigma)-\alpha \sigma \ln 2 \\
\operatorname{var}(X)=\sigma^{2}\left(\frac{\pi^{2}}{6}-\alpha(1+\alpha)(\ln 2)^{2}\right),
\end{gathered}
$$

em que, como anteriormente, $\mathcal{E}$ é a constante de Euler. Obtiveram ainda a equação para gerar números aleatórios desta distribuição e as equações para obtenção do estimador de máxima verossimilhança que não têm solução analítica mas podem ser usadas para obtenção do estimador de máxima verossimilhança através de um método numérico, como por exemplo, o método de Newton Raphson.

O quantil $x_{p}$ é dado por

$$
x_{p}=\mu-\sigma \ln \left(-\ln \left(\frac{1+\alpha-\sqrt{(1+\alpha)^{2}-4 \alpha p}}{2 \alpha}\right)\right)
$$

(ver Apêndice F.3). A variável aleatória $X \sim T E V(\mu, \sigma, \alpha)$ pode, portanto, ser gerada diretamente de $U \sim \operatorname{Unif}(0,1)$ por $X=\mu-\sigma \ln \left(-\ln \left(\left(1+\alpha-\sqrt{(1+\alpha)^{2}-4 \alpha U}\right) / 2 \alpha\right)\right)$.

Os coeficientes de assimetria e de curtose da distribuição $\operatorname{TEV}(\mu, \sigma, \alpha)$ são dadas, respectivamente, por

$$
\gamma_{1, T E V}=\gamma_{1, E V} \frac{1-\left((\ln 2)^{3} / 2 \zeta(3)\right) \alpha(1+\alpha)(1+2 \alpha)}{\left(1-6(\ln 2 / \pi)^{2} \alpha(1+\alpha)\right)^{3 / 2}}
$$

$\mathrm{e}$

$$
\gamma_{2, T E V}-3=\left(\gamma_{2, E V}-3\right) \frac{1-15(\ln 2 / \pi)^{4} \alpha(1+\alpha)(1+6 \alpha(1+\alpha))}{\left(1-6(\ln 2 / \pi)^{2} \alpha(1+\alpha)\right)^{2}},
$$

em que $\zeta(s)=\sum_{k=1}^{\infty} k^{-s}$, Re $s>1$ é a função zeta de Riemann e $\zeta(3) \approx 1,2$.

O mínimo e o máximo do coeficiente de assimetria são 0,718 e 1,321 , para $\alpha=1$ e $\alpha=0,608$, 
respectivamente. O mínimo e o máximo do coeficiente de curtose são 4,052 e 6,703, para $\alpha=1$ e $\alpha=$ 0, 727, respectivamente (ver Apêndice F.3).

A Figura 4.7 apresenta apresenta os gráficos dos coeficientes de assimetria e de curtose da distribuição valor extremo transmutada $T E V(\mu, \sigma, \alpha)$ e podemos observar que é flexível em relação a Gumbel.
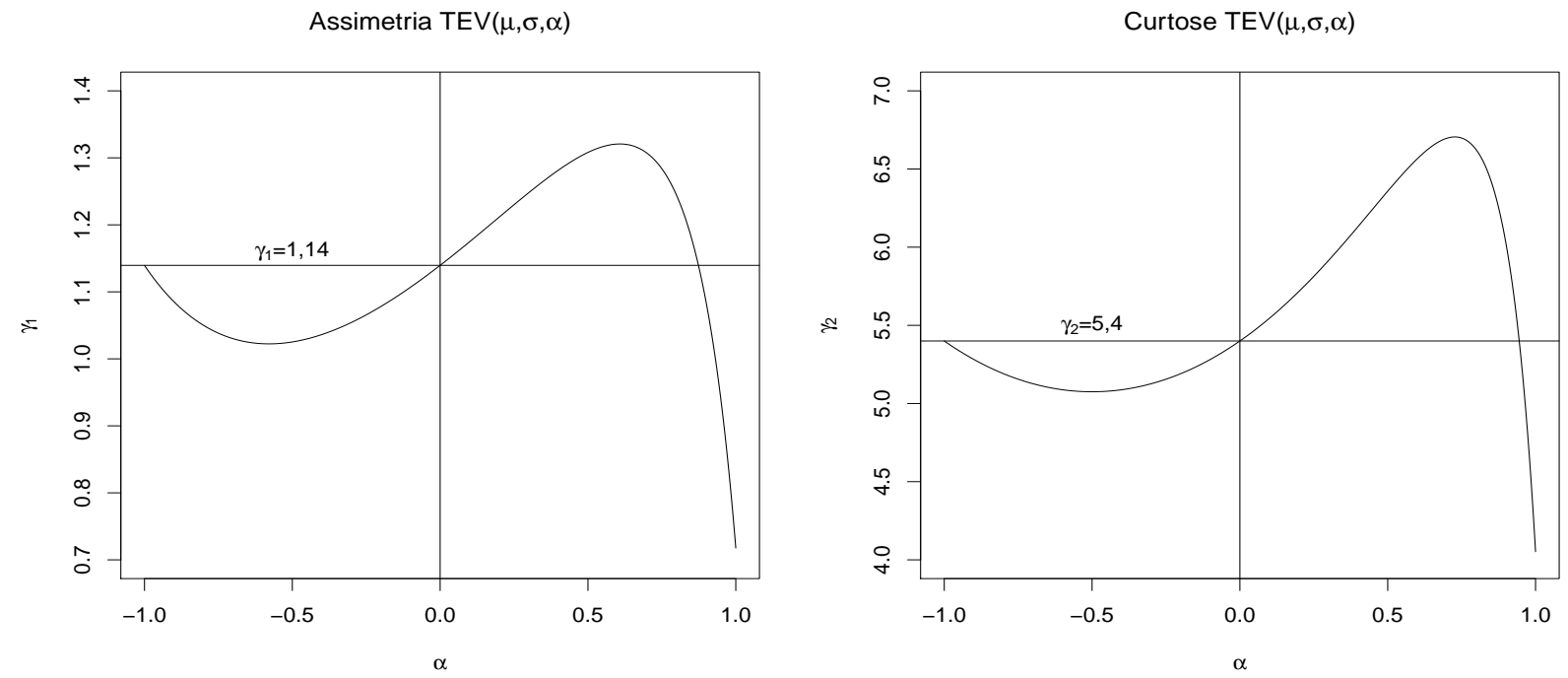

Figura 4.7: Gráficos dos coeficientes de assimetria e de curtose da distribuição valor extremo transmutada $T E V(\mu, \sigma, \alpha)$ em função de $\alpha$.

\subsubsection{Distribuição Kumaraswamy Gumbel}

Cordeiro et al. (2012) definiram uma generalização de uma função de distribuição acumulada $G(x)$ a partir da distribuição Kumaraswamy, a qual denominaram Kum-G. As funções de distribuição acumulada das distribuições Kumaraswamy e Kum-G são definidas, respectivamente, por

$$
F_{K u m}(x ; \alpha, \beta)=1-\left\{1-x^{\alpha}\right\}^{\beta}, \quad x \in(0,1)
$$

e

$$
F_{K u m-G}(x ; \alpha, \beta)=1-\left\{1-G(x)^{\alpha}\right\}^{\beta}, \quad x \in \mathbb{R},
$$

em que $\alpha>0$ e $\beta>0$ são parâmetros adicionais que estão diretamente ligados à assimetria e curtose.

Se a função de distribuição $G(x)$ é a $E V(\mu, \sigma)$, a função de distribuição acumulada é dada por

$$
F_{\text {KumGum }}(x ; \mu, \sigma, \alpha, \beta)=1-\left[1-\left(\exp \left(-\exp \left(-\frac{x-\mu}{\sigma}\right)\right)\right)^{\alpha}\right]^{\beta}, \quad x \in \mathbb{R},
$$

em que $\mu \in \mathbb{R}$ é o parâmetro de localização, $\sigma>0$ o parâmetro de dispersão e $\alpha>0$ e $\beta>0$ são parâmetros relacionados a assimetria e curtose. 
Note que, se $X \sim \operatorname{KumGum}(\mu, \sigma, \alpha, \beta)$ temos que

$$
\begin{aligned}
F_{\text {KumGum }}(x ; \mu, \sigma, \alpha, \beta) & =1-\left[1-\exp \left(-\exp \left(-\left(x-\mu^{*}\right) / \sigma\right)\right)\right]^{\beta} \\
& =F_{\text {KumGum }}\left(x ; \mu^{*}, \sigma, 1, \beta\right)=F_{E G u}\left(x ; \mu^{*}, \sigma, \beta\right)
\end{aligned}
$$

em que $\mu^{*}=\mu+\sigma \ln \alpha$. Logo, a família de distribuições Kumaraswamy Gumbel $\operatorname{KumGum}(\mu, \sigma, \alpha, \beta)$ em que $\mu \in \mathbb{R}, \sigma>0, \alpha>0$ e $\beta>0$ não é identificável e coincide com a família de distribuições Gumbel exponencializada $E G\left(\mu^{*}, \sigma, \beta\right)$ em que $\mu^{*} \in \mathbb{R}, \sigma>0$ e $\beta>0$. Portanto, não será considerada no que segue.

\subsubsection{Distribuição Gumbel generalizada}

Dubey (1969) construiu uma generalização da distribuição valor extremo máximo, que inclui um terceiro parâmetro na distribuição e supõe que o parâmetro tem distribuição gama $(\alpha, \beta)$, resultando na distribuição formalmente denominada Gumbel generalizada com quatro parâmetros $\mu, \sigma, \alpha$, $\beta$, digamos $G G u(\mu, \sigma, \alpha, \beta)$, cuja função de distribuição acumulada é dada por

$$
F_{G G u}(x ; \mu, \sigma, \alpha, \beta)=\left(1+\frac{\sigma}{\beta} \exp \left(-\frac{x-\mu}{\sigma}\right)\right)^{-\alpha}, \quad x \in \mathbb{R}
$$

em que $\mu \in \mathbb{R}$ é o parâmetro de localização, $\sigma>0$ o parâmetro de dispersão, $\alpha>0$ e $\beta>0$. Esta distribuição pode ser vista como uma distribuição logística generalizada, foi inicialmente citada em Hald (1952) e é, usualmente, denominada distribuição logística generalizada tipo 1.

Temos que

$$
F_{G G u}(x ; \mu, \sigma, \alpha, \beta)=\left(1+\frac{1}{\alpha} \exp \left(-\frac{x-\mu^{*}}{\sigma}\right)\right)^{-\alpha}, \quad x \in \mathbb{R},
$$

em que $\mu^{*}=\mu+\sigma \ln \left(\sigma \alpha \beta^{-1}\right) \in \mathbb{R}, \sigma>0$ o parâmetro de dispersão, $\alpha>0$ e $\beta>0$. Logo a família de distribuições $G G u(\mu, \sigma, \alpha, \beta)$, em que $\mu \in \mathbb{R}, \sigma>0, \alpha>0$ e $\beta>0$, coincide com uma família de distribuições com três parâmetros, digamos, $G G u 3(\mu, \sigma, \alpha)$.

Seja $X$ uma variável aleatória com distribuição Gumbel generalizada com três parâmetros, $\mu, \sigma$ e $\alpha$, $X \sim G G u 3(\mu, \sigma, \alpha)$. Sua função densidade de probabilidade é dada por

$$
f_{G G u 3}(x ; \mu, \sigma, \alpha)=\frac{1}{\sigma}\left(1+\frac{1}{\alpha} \exp \left(-\frac{x-\mu}{\sigma}\right)\right)^{-\alpha-1} \exp \left(-\frac{x-\mu}{\sigma}\right), \quad x \in \mathbb{R},
$$

e função de distribuição acumulada dada por

$$
F_{G G u 3}(x ; \mu, \sigma, \alpha, \beta)=\left(1+\frac{1}{\alpha} \exp \left(-\frac{x-\mu}{\sigma}\right)\right)^{-\alpha}, \quad x \in \mathbb{R},
$$

em que $\mu \in \mathbb{R}$ é o parâmetro de localização, $\sigma>0$ o parâmetro de dispersão e $\alpha>0$. A distribuição valor extremo máximo tipo I ou Gumbel generalizada com três parâmetros tem como caso limite a distribuição valor extremo máximo quando $\alpha \rightarrow \infty$. A Figura 4.8 apresenta algumas densidades da distribuição valor extremo máximo tipo I ou Gumbel generalizada com três parâmetros com seus valores correspondentes de 
$\alpha$.

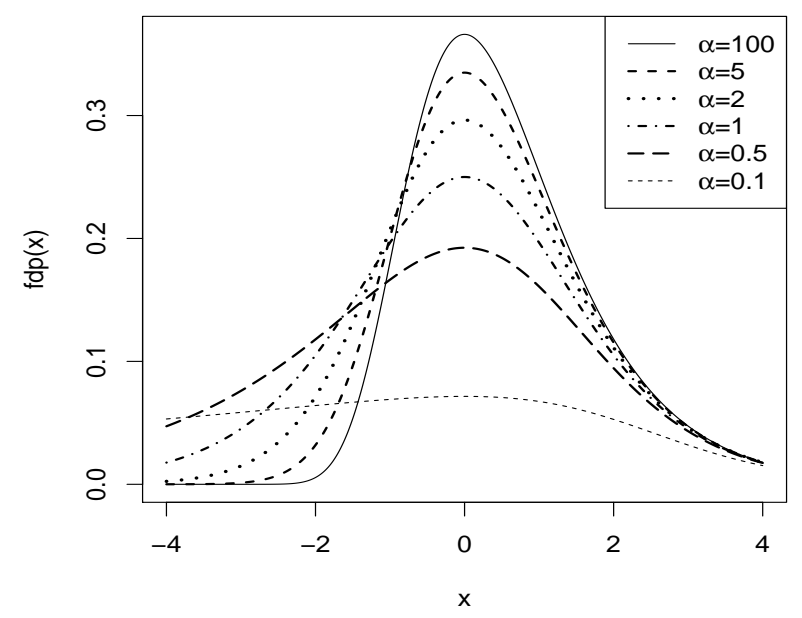

Figura 4.8: Função densidade de probabilidade de distribuição valor extremo máximo tipo I ou Gumbel generalizada com três parâmetros $G G u 3(0,1, \alpha)$.

A esperança e variância são dadas, respectivamente, por

$$
\mathrm{E}(X)=\mu-\sigma(-\mathcal{E}+\ln (\alpha)-\psi(\alpha))
$$

e

$$
\operatorname{var}(X)=\sigma^{2}\left(\pi^{2} / 6+\psi^{\prime}(\alpha)\right)
$$

em que $\mathcal{E}$ é a constante de Euler e $\psi(x)=d(\ln \Gamma(x)) / d x=\Gamma^{\prime}(x) / \Gamma(x)$ é a função digama (ver Apêndice F.4).

O quantil $x_{p}$ é dado por

$$
x_{p}=\mu-\sigma \ln \left(\alpha\left(p^{-1 / \alpha}-1\right)\right) .
$$

A variável aleatória $X \sim G G u 3(\mu, \sigma, \alpha)$ pode, portanto, ser gerada diretamente de $U \sim U n i f(0,1)$, por $X=\mu-\sigma \ln \left(\alpha\left(U^{-1 / \alpha}-1\right)\right)$.

Os coeficientes de assimetria e de curtose da distribuição $G G u 3(\mu, \sigma, \alpha)$ são dados, respectivamente, por

$$
\gamma_{1, \mathrm{GGu} 3}=\frac{\psi^{\prime \prime}(\alpha)-\psi^{\prime \prime}(1)}{\left(\psi^{\prime}(1)+\psi^{\prime}(\alpha)\right)^{3 / 2}}
$$

$\mathrm{e}$

$$
\gamma_{2, \mathrm{GGu} 3}=\frac{\psi^{\prime \prime \prime}(1)+\psi^{\prime \prime \prime}(\alpha)}{\left(\psi^{\prime}(1)+\psi^{\prime}(\alpha)\right)^{2}}+3,
$$

O mínimo e o máximo dos coeficientes de assimetria e de curtose são, respectivamente, -2, 1,14, 4,15 e 9 (ver Apêndice F.4).

A Figura 4.9 apresenta apresenta os gráficos dos coeficientes de assimetria e de curtose da distribuição 
valor extremo máximo tipo I ou Gumbel com três parâmetros $G G u 3(\mu, \sigma, \alpha)$ e podemos observar que a assimetria é sempre inferior a da Gumbel enquanto a curtose pode ser superior.
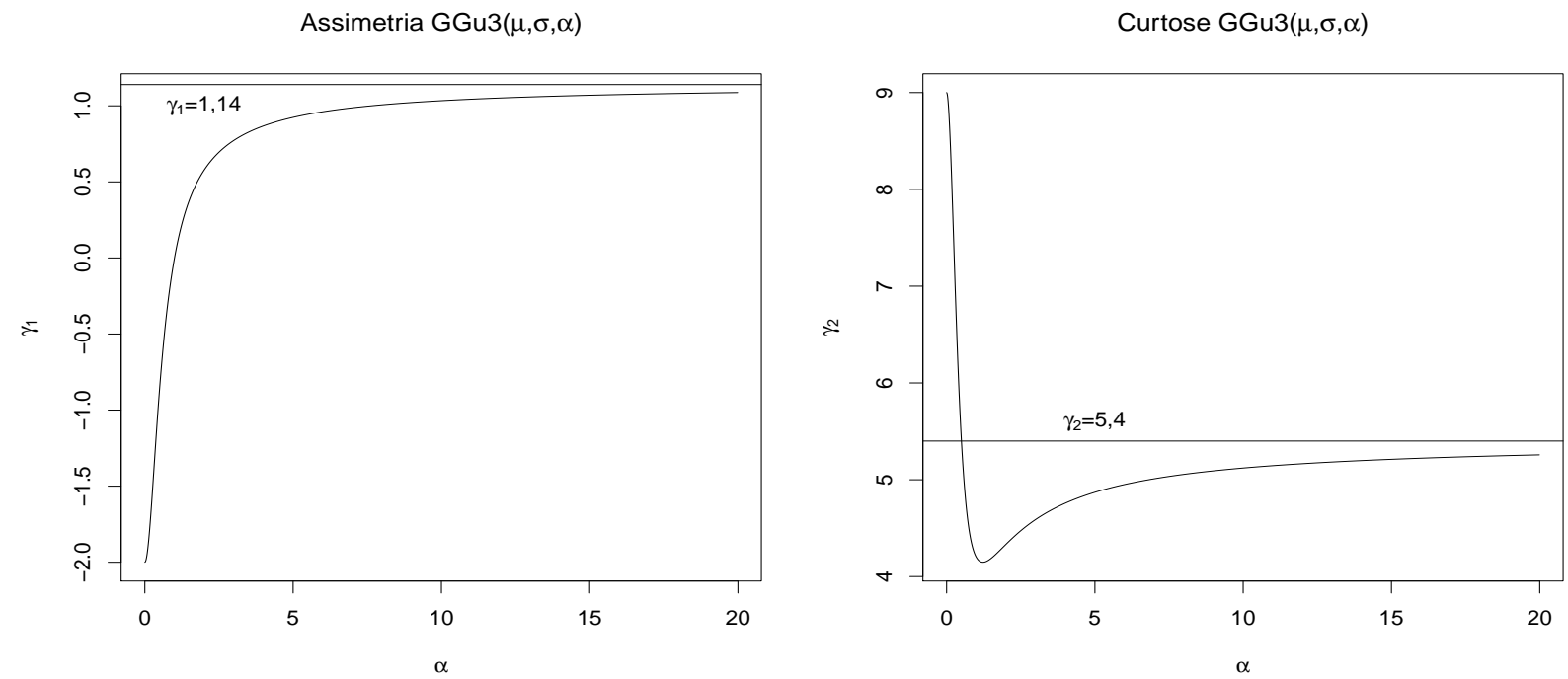

Figura 4.9: Gráficos dos coeficientes de assimetria e de curtose da distribuição Gumbel generalizada com três parâmetros $G G u 3(\mu, \sigma, \alpha)$ em função de $\alpha$.

\subsubsection{Distribuição exponencial-gama com três parâmetros}

Ojo (2001) comenta que propusera uma primeira generalização da distribuição Gumbel com três parâmetros, aqui denominada distribuição exponencial-gama com três parâmetros $\operatorname{Exp} \operatorname{Gama3}(\mu, \sigma, \alpha)$, cujas função densidade de probabilidade e função de distribuição acumulada são dadas, respectivamente, por

$$
f_{\operatorname{ExpGama3}}(x ; \mu, \sigma, \alpha)=\frac{1}{\Gamma(\alpha)} \frac{1}{\sigma} \exp \left(-\exp \left(-\frac{x-\mu}{\sigma}\right)\right) \exp \left(-\alpha \frac{x-\mu}{\sigma}\right), \quad x \in \mathbb{R}
$$

$\mathrm{e}$

$$
F_{\operatorname{ExpGama3}}(\mu, \sigma, \alpha)=\frac{1}{\Gamma(\alpha)} \Gamma\left(\alpha, \exp \left(-\frac{x-\mu}{\sigma}\right)\right), \quad x \in \mathbb{R},
$$

em que $\mu \in \mathbb{R}, \sigma>0$ e $\alpha>0$ e $\Gamma(s, x)=\int_{x}^{\infty} t^{s-1} \exp (-t) d t$ é a função gama incompleta (ver Apêndice F.5). A distribuição exponencial-gama com três parâmetros tem como caso particular a distribuição Gumbel quando $\alpha=1$.

A Figura 4.10 apresenta a função densidade de probabilidade de uma distribuição $\operatorname{Exp} \operatorname{Gama3}(0,1, \alpha)$ para alguns valores de $\alpha$.

A esperança e variância são dadas, respectivamente, por

$$
\mathrm{E}(X)=\mu-\sigma \psi(\alpha)
$$

e

$$
\operatorname{var}(X)=\sigma^{2} \psi^{\prime}(\alpha)
$$




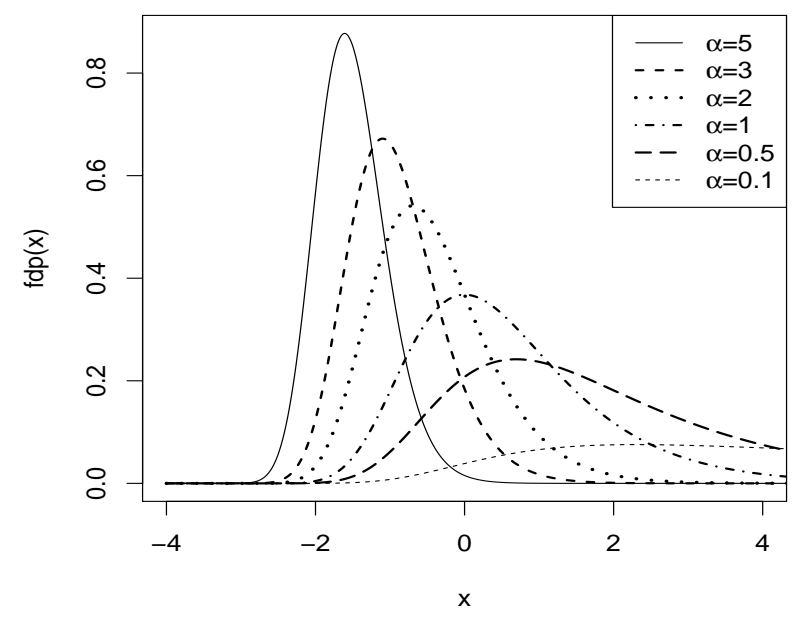

Figura 4.10: Função densidade de probabilidade da distribuição ExpGama3(0,1, $\alpha)$.

em que $\psi(x)=d(\ln \Gamma(x)) / d x=\Gamma^{\prime}(x) / \Gamma(x)$ é a função digama (ver Apêndice F.5).

O quantil $x_{p}$ tal que $F\left(x_{p}\right)=p$ não pode ser obtido analiticamente. Para estimar os quantis pode-se gerar uma amostra suficientemente grande e obter os quantis amostrais. A variável aleatória $X \sim \operatorname{Exp} \operatorname{Gama3}(\mu, \sigma, \alpha)$ pode ser gerada diretamente de uma distribuição gama com parâmetros $\alpha$ e 1, digamos, $Y \sim \operatorname{gama}(\alpha, 1)$, por $X=\mu-\sigma \ln (Y)$. Aqui consideramos $W \sim \operatorname{gama}(\alpha, \beta)$ se $f(w)=\left(\beta^{\alpha} / \Gamma(\alpha)\right) w^{\alpha-1} \exp (-\beta w)$.

Os coeficientes de assimetria $\left(\gamma_{1}\right)$ e de curtose $\left(\gamma_{2}\right)$ da distribuição $\operatorname{Exp} \operatorname{Gama3}(\mu, \sigma, \alpha)$ são dados, respectivamente, por

$$
\gamma_{1, \text { ExpGama3 }}=-\frac{\psi^{\prime \prime}(\alpha)}{\psi^{\prime}(\alpha)^{3 / 2}}
$$

e

$$
\gamma_{2, \text { ExpGama3 }}=\frac{\psi^{\prime \prime \prime}(\alpha)}{\psi^{\prime}(\alpha)^{2}}+3
$$

em que $\psi^{(n)}(x)$ é a $n$-ésima derivada da função digama $\psi(x)$. O mínimo e o máximo dos coeficientes de assimetria $\left(\gamma_{1}\right)$ e de curtose $\left(\gamma_{2}\right)$ são, respectivamente, 0, 2, 3 e 9 (ver Apêndice F.5).

A Figura 4.11 apresenta apresenta os gráficos dos coeficientes de assimetria e de curtose da distribuição exponencial-gama com três parâmetros $\operatorname{Exp} \operatorname{Gama} 3(\mu, \sigma, \alpha)$ e podemos observar que é flexível em relação a Gumbel.

\subsubsection{Distribuição exponencial-gama}

Gumbel (1935) obteve a distribuição assintótica da $r$-ésima estatística de ordem, em que $r=1$ corresponde ao máximo, de uma distribuição $E V(0,1)$, que tem função densidade de probabilidade

$$
f(x ; \mu, \sigma, \alpha)=\frac{r^{r}}{\Gamma(r)} \exp (-r \exp (-x)) \exp (-r x), \quad x \in \mathbb{R} .
$$



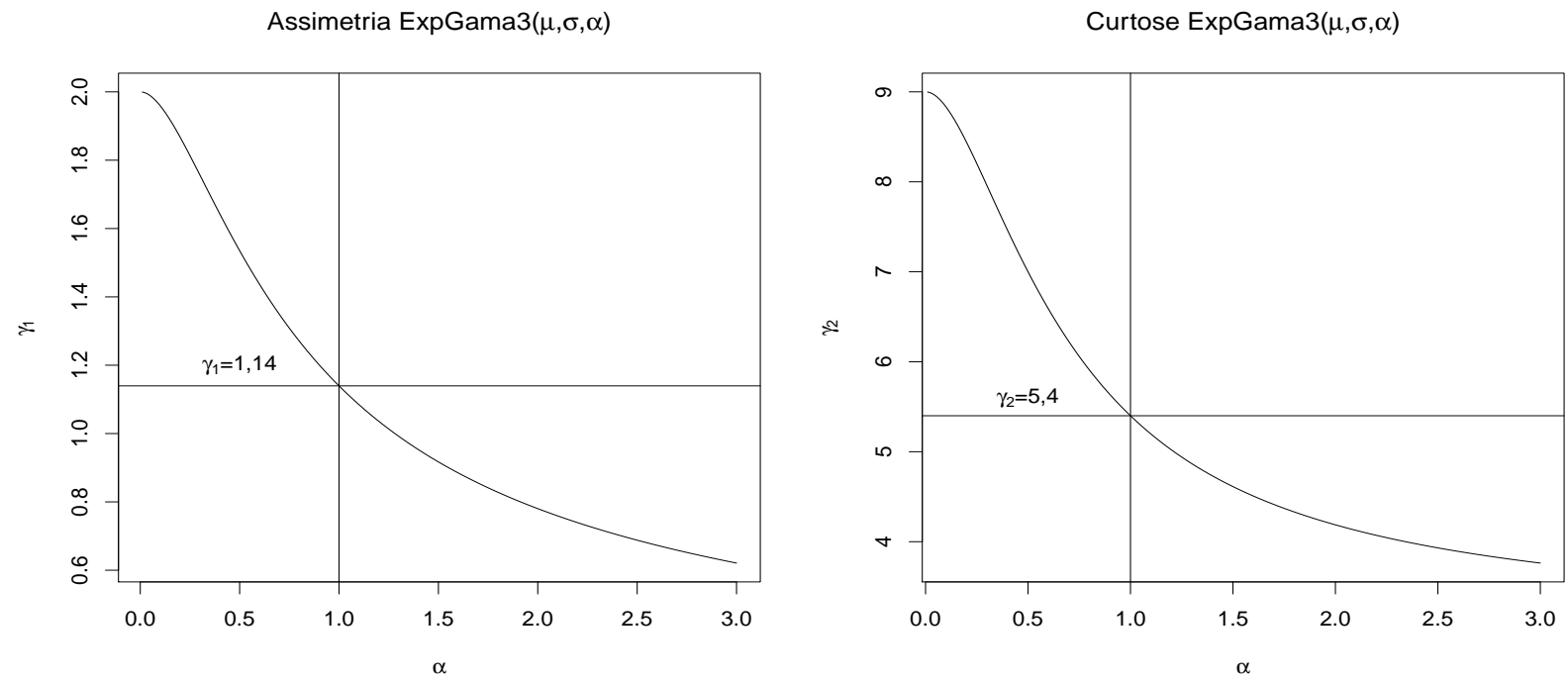

Figura 4.11: Gráficos dos coeficientes de assimetria e de curtose da distribuição exponencial-gama com três parâmetros $\operatorname{Exp} \operatorname{Gama3}(\mu, \sigma, \alpha)$ em função de $\alpha$.

Quando $r=1$ a distribuição se iguala a distribuição Gumbel. Adeyemi \& Ojo (2003) propuseram esta distribuição como uma generalização da distribuição Gumbel, sendo $r$ um parâmetro de forma. Obtiveram os cumulantes e a relação de tal distribuição com a distribuição Erlang e com a distribuição logística generalizada. Uma forma mais geral, usualmente denominada de distribuição exponencial-gama, $\operatorname{Exp} \operatorname{Gama}(\mu, \sigma, \alpha, \beta)$ (ver Balakrishnan \& Leung, 1988, p. 34), é definida pela função densidade de probabilidade e função de distribuição acumulada, respectivamente,

$$
f_{\text {ExpGama }}(x ; \mu, \sigma, \alpha, \beta)=\frac{\alpha^{\beta}}{\sigma \Gamma(\beta)} \exp \left(-\alpha \exp \left(-\frac{x-\mu}{\sigma}\right)\right) \exp \left(-\beta \frac{x-\mu}{\sigma}\right), \quad x \in \mathbb{R}
$$

e

$$
F_{\text {ExpGama }}(x ; \mu, \sigma, \alpha, \beta)=\frac{1}{\Gamma(\beta)} \Gamma\left(\beta, \alpha \exp \left(-\frac{x-\mu}{\sigma}\right)\right), \quad x \in \mathbb{R}
$$

em que $\mu \in \mathbb{R}, \sigma>0, \alpha \in \mathbb{R}$ e $\beta>0$ (ver Apêndice F.6). Quando $\alpha=\beta=1$ a distribuição exponencialgama coincide com a distribuição Gumbel e quando $\alpha=1$ coincide com a distribuição exponencial-gama com três parâmetros (ver Seção 4.2.6).

Se $X \sim \operatorname{Exp} \operatorname{Gama}(\mu, \sigma, \alpha, \beta)$ temos que

$$
F_{E x p G a m a}(x ; \mu, \sigma, \alpha, \beta)=\frac{\Gamma\left(\beta, \exp \left(-\frac{x-\mu^{*}}{\sigma}\right)\right)}{\Gamma(\beta)}=F_{E x p G a m a}\left(x ; \mu^{*}, \sigma, 1, \beta\right)=F_{E x p G a m a 3}\left(x ; \mu^{*}, \sigma, \beta\right),
$$

em que $\mu^{*}=\mu+\sigma \ln \alpha \in \mathbb{R}$. Logo, a família de distribuições exponencial-gama $\operatorname{Exp} \operatorname{Gama}(\mu, \sigma, \alpha, \beta)$, em que $\mu \in \mathbb{R}, \sigma>0, \alpha>0$ e $\beta>0$ não é identificável e coincide com a família de distribuições exponencial-gama com três parâmetros $\operatorname{Exp} \operatorname{Gama} 3(\mu, \sigma, \beta)$, em que $\mu \in \mathbb{R}, \sigma>0$ e $\beta>0$. Portanto, não 
será considerada no que segue.

\subsubsection{Distribuição logística generalizada tipo IV}

A distribuição logística generalizada tipo IV foi proposta em Prentice (1975). Seja $X$ uma variável aleatória com distribuição logística generalizada tipo IV, $X \sim G L I V(\mu, \sigma, \alpha, \beta)$. A função densidade de probabilidade e a função de distribuição acumulada são definidas, respectivamente, por

$$
f_{G L I V}(x ; \mu, \sigma, \alpha, \beta)=\left(\frac{\alpha}{\beta}\right)^{\alpha} \frac{1}{\sigma B(\alpha, \beta)} \frac{[\exp (-(x-\mu) / \sigma)]^{\alpha}}{[1+(\alpha / \beta) \exp (-(x-\mu) / \sigma)]^{\alpha+\beta}}, \quad x \in \mathbb{R}
$$

$\mathrm{e}$

$F_{G L I V}(x ; \mu, \sigma, \alpha)=\frac{1}{\beta B(\alpha, \beta)}\left(\frac{\beta}{\alpha} \exp \left(\frac{x-\mu}{\sigma}\right)\right)_{2}^{\beta} F_{1}\left(\beta, \alpha+\beta ; 1+\beta ;-\frac{\beta}{\alpha} \exp \left(\frac{x-\mu}{\sigma}\right)\right), x \in \mathbb{R}$

em que $\mu \in \mathbb{R}$ é o parâmetro de localização, $\sigma>0$ o parâmetro de dispersão, $\alpha>0$ e $\beta>0$ e ${ }_{2} F_{1}(a, b ; c ; z)=(\Gamma(c) /(\Gamma(b) \Gamma(c-b))) \int_{0}^{1} t^{b-1}(1-t)^{c-b-1} /(1-t z)^{a}$ é a função hipergeométrica (ver Apêndice F.7). A distribuição logística generalizada tipo IV, $G L I V(\mu, \sigma, \alpha, \beta)$, tem como caso particular a distribuição Gumbel, $E V(\mu, \sigma)$, quando $\alpha=1$ e $\beta \rightarrow \infty$ e a distribuição Gumbel generalizada com três parâmetros , $G G u 3(\mu, \sigma, \beta)$, quando $\alpha=1$. A Figura 4.12 apresenta a função densidade de probabilidade de uma distribuição logística generalizada tipo IV, $\operatorname{GLIV}(0,1, \alpha, \beta)$, para alguns valores de $\alpha$ e $\beta$.

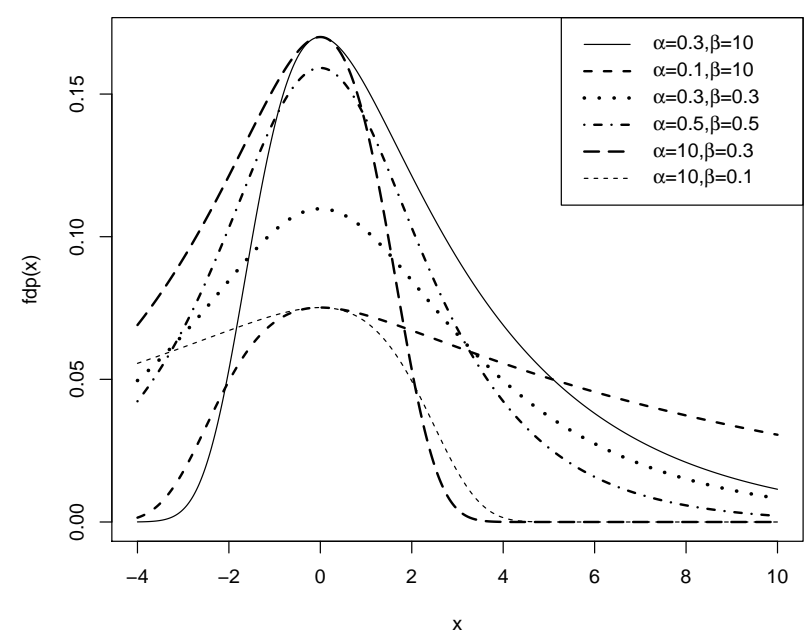

Figura 4.12: Função densidade de probabilidade da distribuição $\operatorname{GLIV}(0,1, \alpha, \beta)$.

A esperança e variância são dadas, respectivamente, por

$$
\mathrm{E}(X)=\mu+\sigma\left(\psi(\beta)-\psi(\alpha)-\ln \frac{\beta}{\alpha}\right)
$$


$\mathrm{e}$

$$
\operatorname{var}(X)=\sigma^{2}\left(\psi^{\prime}(\beta)+\psi^{\prime}(\alpha)\right),
$$

em que $\psi(x)=d(\ln \Gamma(x)) / d x=\Gamma^{\prime}(x) / \Gamma(x)$ é a função digama (ver Apêndice F.7).

O quantil $x_{p}$ não pode ser obtido analiticamente. Para estimar os quantis pode-se gerar uma amostra suficientemente grande e obter os quantis amostrais. A variável aleatória $X \sim G L I V(\mu, \sigma, \alpha, \beta)$ pode ser gerada diretamente de uma distribuição $F$ com parâmetros $2 \alpha$ e $2 \beta$, digamos, $Y \sim F(2 \alpha, 2 \beta)$, por $x=\mu-\sigma \ln Y$. Aqui consideramos $W \sim F(a, b)$ se $f(w)=(1 / B(a, b))(a / b)^{a} w^{a-1} /(1+(a / b) w)^{(a+b)}$ (ver Apêndice F.7). A variável aleatória $X \sim G L I V(\mu, \sigma, \alpha, \beta)$ também pode ser gerada diretamente de uma distribuição Beta de segundo tipo, digamos, $Y \sim \operatorname{BetaI}(1, \alpha, \beta)$, por $x=\mu-\sigma \ln ((\beta / \alpha) Y)$. Aqui, $W \sim \operatorname{BetaII}(b, p, q)$ se a densidade é $f(w)=\left(w^{p-1}\right) /\left(b^{p} B(p, q)(1+w / b)^{p+q}\right)$.

Os coeficientes de assimetria e de curtose da distribuição logística generalizada tipo IV $G L I V(\mu, \sigma, \alpha, \beta)$ são dadas, respectivamente, por

$$
\gamma_{1, G L I V}(X)=\frac{\psi^{\prime \prime}(\beta)-\psi^{\prime \prime}(\alpha)}{\left(\psi^{\prime}(\beta)+\psi^{\prime}(\alpha)\right)^{3 / 2}} \quad \text { e } \quad \gamma_{2, G L I V}(X)=\frac{\psi^{\prime \prime \prime}(\alpha)+\psi^{\prime \prime \prime}(\beta)}{\left(\psi^{\prime}(\beta)+\psi^{\prime}(\alpha)\right)^{2}}+3
$$

O mínimo e o máximo dos coeficientes de assimetria e de curtose são, respectivamente, -2, 2, 3 e 9 (ver Apêndice F.7).

A Figura 4.13 apresenta apresenta os gráficos dos coeficientes de assimetria e de curtose da distribuição exponencial-gamma $\operatorname{GLIV}(\mu, \sigma, \alpha, \beta)$ e podemos observar que é flexível em relação a Gumbel.

Assimetria de $\operatorname{GLIV}(\mu, \sigma, \alpha, \beta)$

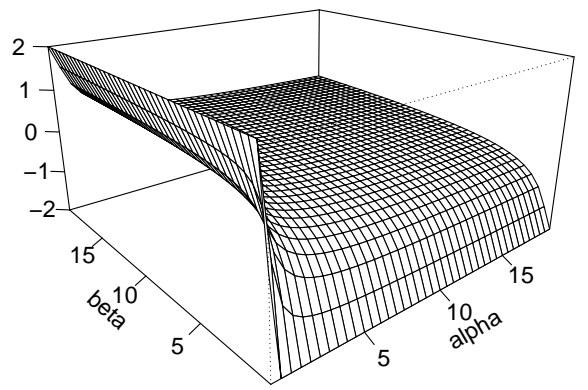

Curtose de $\operatorname{GLIV}(\mu, \sigma, \alpha, \beta)$

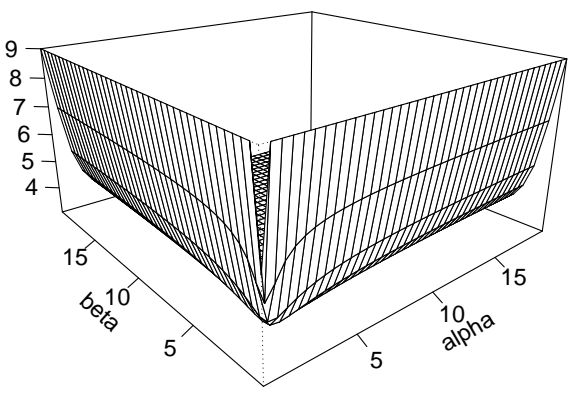

Figura 4.13: Gráficos dos coeficientes de assimetria e de curtose da distribuição logística generalizada tipo IV $\operatorname{GLIV}(\mu, \sigma, \alpha, \beta)$ em função de $\alpha$ e $\beta$.

Em Prentice (1976), esta função densidade de probabilidade é apresentada, de forma simplificada, como

$$
f_{G L I V}(x ; \mu, \sigma, \alpha, \beta)=\frac{1}{\sigma B(\alpha, \beta)} \frac{[\exp (-(x-\mu) / \sigma)]^{\alpha}}{[1+\exp (-(x-\mu) / \sigma)]^{\alpha+\beta}}, \quad x \in \mathbb{R},
$$


em que $\mu \in \mathbb{R}$ é o parâmetro de localização, $\sigma>0$ o parâmetro de dispersão, $\alpha>0$ e $\beta>0$.

A distribuição logística generalizada tipo III, proposta em Balakrishnan \& Leung (1988, p. 34) é um caso particular da distribuição logística generalizada tipo IV, quando $\alpha=\beta$ e a densidade se torna simétrica em torno de $x=\mu$. Sendo assim, não será contemplada neste trabalho, que visa distribuições com caudas direitas mais pesadas do que a distribuição Gumbel.

\subsubsection{Distribuição Gumbel exponencializada generalizada}

Cordeiro et al. (2013) propuseram uma classe de distribuições denominada exponencializada generalizada (EG), generalização da classe de distribuições tipo exponencializada, definida por

$$
F(x)=\left[1-\{1-G(x)\}^{\alpha}\right]^{\beta}
$$

em que $\alpha>0$ e $\beta>0$ são dois parâmetros de forma adicionais e $G(x)$ é uma função de distribuição acumulada contínua. Quando $G(x)$ é a função de distribuição acumulada de uma distribuição Gumbel temos a distribuição Gumbel exponencializada generalizada (EGGu). Seja $X$ uma variável aleatória com distribuição Gumbel exponencializada generalizada, $X \sim E G G u(\mu, \sigma, \alpha, \beta)$. A função densidade de probabilidade e a função de distribuição acumulada são dadas, respectivamente, por

$$
\begin{aligned}
f_{E G G u}(x ; \mu, \sigma, \alpha, \beta)= & \frac{\alpha \beta}{\sigma}\left[1-\left(1-\exp \left(-\exp \left(-\frac{x-\mu}{\sigma}\right)\right)\right)^{\alpha}\right]^{\beta-1} \\
& \times\left[1-\left(\exp \left(-\exp \left(-\frac{x-\mu}{\sigma}\right)\right)\right)\right]^{\alpha-1} \\
& \times \exp \left(-\exp \left(-\frac{x-\mu}{\sigma}\right)\right) \exp \left(-\frac{x-\mu}{\sigma}\right), \quad x \in \mathbb{R}
\end{aligned}
$$

$\mathrm{e}$

$$
F_{E G G u}(x ; \mu, \sigma, \alpha, \beta)=\left[1-\left(1-\exp \left(-\exp \left(-\frac{x-\mu}{\sigma}\right)\right)\right)^{\alpha}\right]^{\beta}, \quad x \in \mathbb{R},
$$

em que $\mu \in \mathbb{R}$ é o parâmetro de localização, $\sigma>0$ o parâmetro de dispersão, $\alpha>0$ e $\beta>0$. A distribuição Gumbel exponencializada generalizada, $\operatorname{EGGu}(\mu, \sigma, \alpha, \beta)$, tem como caso particular a distribuição Gumbel exponencializada, $E G u(\mu, \sigma, \alpha)$, quando $\beta=1$. Note ainda que, se $X \sim E G G u(\mu, \sigma, 1, \beta)$, temos que

$$
F_{E G G u}(x ; \mu, \sigma, 1, \beta)=\exp (-\exp (-(x-(\mu+\sigma \ln \beta)) / \sigma))=F_{E G G u}\left(x ; \mu^{*}, \sigma, 1,1\right)=F_{E V}\left(x ; \mu^{*}, \sigma\right),
$$

em que $\mu^{*}=\mu+\sigma \ln \beta$. Logo, a família de distribuições $E G G u(\mu, \sigma, 1, \beta) \operatorname{com} \mu \in \mathbb{R}, \sigma>0, \alpha=1$ e $\beta>0$ não é identificável e coincide com a família de distribuições $E V\left(\mu^{*}, \sigma\right) \operatorname{com} \mu^{*} \in \mathbb{R}$ e $\sigma>0$. Portanto, não será considerada no que segue.

\subsubsection{Distribuição beta Gumbel}

Nadarajah \& Kotz (2004) propuseram uma generalização da distribuição Gumbel, a qual denominaram 
beta Gumbel (BG), a partir de uma classe de distribuições definida por

$$
F(x)=\frac{B_{G(x)}(\alpha, \beta)}{B(\alpha, \beta)}
$$

para $\alpha>0$ e $\beta>0$, em que $G(x)$ é a função de distribuição acumulada de uma variável aleatória, $B(\alpha, \beta)$ é a função beta e

$$
B_{w}(a, b)=\int_{0}^{w} t^{a-1}(1-t)^{b-1} d t
$$

é a função beta incompleta. Seja $X$ uma variável aleatória com distribuição beta Gumbel, $X \sim$ $B G(\mu, \sigma, \alpha, \beta)$. A função de distribuição acumulada é dada por

$$
F_{B G}(x ; \mu, \sigma, \alpha, \beta)=\int_{0}^{\exp (-\exp (-(x-\mu) / \sigma))} t^{\alpha-1}(1-t)^{\beta-1} d t, \quad x \in \mathbb{R},
$$

em que $\mu \in \mathbb{R}$ é o parâmetro de localização, $\sigma>0$ o parâmetro de dispersão, $\alpha>0$ e $\beta>0$. A distribuição beta Gumbel, $B G(\mu, \sigma, \alpha, \beta)$, tem como caso particular a distribuição Gumbel exponencializada, $E G u(\mu, \sigma, \alpha)$, quando $\alpha=1$ e a distribuição Gumbel, $E V(\mu, \sigma)$, quando $\beta=1$. Note ainda que, se $X \sim B G(\mu, \sigma, \alpha, 1)$, temos que

$$
F_{B G}(x ; \mu, \sigma, \alpha, 1)=\int_{0}^{\exp (-\exp (-(x-\mu) / \sigma))} t^{\alpha-1} d t=F_{B G}\left(x ; \mu^{*}, \sigma, 1,1\right)=F_{E V}\left(x ; \mu^{*}, \sigma\right),
$$

em que $\mu^{*}=\mu+\sigma \ln \alpha$. Logo, a família de distribuições $B G(\mu, \sigma, \alpha, 1) \operatorname{com} \mu \in \mathbb{R}, \sigma>0, \alpha>0$ e $\beta=1$ não é identificável e coincide com a família de distribuições $E V\left(\mu^{*}, \sigma\right)$ com $\mu^{*} \in \mathbb{R}$ e $\sigma>0$. Portanto, não será considerada no que segue.

\subsubsection{Distribuição Kummer beta generalizada Gumbel}

Pescim et al. (2012) propuseram uma generalização da distribuição Gumbel, a qual denominaram beta Gumbel (BG), a partir de uma classe de distribuições definida por

$$
F(x)=\frac{B_{G(x)}(\alpha, \beta)}{B(\alpha, \beta)}
$$

para $\alpha>0$ e $\beta>0$, em que $G(x)$ é a função de distribuição acumulada de uma variável aleatória, $B(\alpha, \beta)$ é a função beta e

$$
B_{w}(a, b)=\int_{0}^{w} t^{a-1}(1-t)^{b-1} d t
$$

é a função beta incompleta. Seja $X$ uma variável aleatória com distribuição beta Gumbel, $X \sim$ $B G(\mu, \sigma, \alpha, \beta)$. A função de distribuição acumulada é dada por

$$
F_{B G}(x ; \mu, \sigma, \alpha, \beta)=\int_{0}^{\exp (-\exp (-(x-\mu) / \sigma))} t^{\alpha-1}(1-t)^{\beta-1} d t, \quad x \in \mathbb{R},
$$

em que $\mu \in \mathbb{R}$ é o parâmetro de localização, $\sigma>0$ o parâmetro de dispersão, $\alpha>0$ e $\beta>0$. A dis- 
tribuição beta Gumbel, $B G(\mu, \sigma, \alpha, \beta)$, tem como caso particular a distribuição Gumbel exponencializada, $E G u(\mu, \sigma, \alpha)$, quando $\alpha=1$ e a distribuição Gumbel, $E V(\mu, \sigma)$, quando $\beta=1$. Note ainda que, se $X \sim B G(\mu, \sigma, \alpha, 1)$, temos que

$$
F_{B G}(x ; \mu, \sigma, \alpha, 1)=\int_{0}^{\exp (-\exp (-(x-\mu) / \sigma))} t^{\alpha-1} d t=F_{B G}\left(x ; \mu^{*}, \sigma, 1,1\right)=F_{E V}\left(x ; \mu^{*}, \sigma\right),
$$

em que $\mu^{*}=\mu+\sigma \ln \alpha$. Logo, a família de distribuições $B G(\mu, \sigma, \alpha, 1) \operatorname{com} \mu \in \mathbb{R}, \sigma>0, \alpha>0$ e $\beta=1$ não é identificável e coincide com a família de distribuições $E V\left(\mu^{*}, \sigma\right)$ com $\mu^{*} \in \mathbb{R}$ e $\sigma>0$. Portanto, não será considerada no que segue.

\subsubsection{Mistura de dois componentes de distribuição valor extremo máximo}

Rossi et al. (1986) propuseram uma mistura de dois componentes de distribuição valor extremo máximo (TCEV) para analisar frequência de inundações, cuja função densidade de probabilidade e função de distribuição acumulada são, respectivamente,

$$
\begin{aligned}
& f_{T C E V}\left(x ; \mu_{1}, \sigma_{1}, \mu_{2}, \sigma_{2}, \alpha\right)= \\
& \frac{1-\alpha}{\sigma_{1}} \exp \left(-\frac{x-\mu_{1}}{\sigma_{1}}\right) \exp \left(-\exp \left(-\frac{x-\mu_{1}}{\sigma_{1}}\right)\right)+\frac{\alpha}{\sigma_{2}} \exp \left(-\frac{x-\mu_{2}}{\sigma_{2}}\right) \exp \left(-\exp \left(-\frac{x-\mu_{2}}{\sigma_{2}}\right)\right), \\
& x \in \mathbb{R},
\end{aligned}
$$

e

$F_{T C E V}\left(x ; \mu_{1}, \sigma_{1}, \mu_{2}, \sigma_{2}, \alpha\right)=(1-\alpha) \exp \left(-\exp \left(-\frac{x-\mu_{1}}{\sigma_{1}}\right)\right)+\alpha \exp \left(-\exp \left(-\frac{x-\mu_{2}}{\sigma_{2}}\right)\right), x \in \mathbb{R}$,

em que $\mu_{1} \in \mathbb{R}$ e $\mu_{2} \in \mathbb{R}$ são parâmetros de localização, $\sigma_{1}>0$ e $\sigma_{2}>0$ são parâmetros de dispersão e $0<\alpha<1$.

Se $0<\alpha<1$, a mistura de dois componentes da distribuição Gumbel não é identificável pois $F\left(x ; \mu_{1}, \sigma_{1}, \mu_{2}, \sigma_{2}, \alpha\right)=F\left(x ; \mu_{2}, \sigma_{2}, \mu_{1}, \sigma_{1},(1-\alpha)\right)$. A falta de identificabilidade devido a permutação dos componentes de uma mistura de distribuições de uma mesma família é geralmente resolvida pela imposição de restrições aos parâmetros. Aqui, basta considerar $0<\alpha<0,5$ para obter identificabilidade ${ }^{2}$. Quando $\alpha \rightarrow 0$ a mistura de dois componentes de distribuição Gumbel, com $0<\alpha<0,5$, coincide com a distribuição Gumbel.

A Figura 4.14 apresenta a função densidade de probabilidade de uma distribuição mistura de dois componentes de distribuição valor extremo máximo, $\operatorname{TCEV}(0,1,10,5, \alpha)$, para alguns valores de $\alpha$.

Sejam $X_{1} \sim E V\left(\mu_{1}, \sigma_{1}\right), X_{2} \sim E V\left(\mu_{2}, \sigma_{2}\right), X=X_{1}$ com probabilidade $(1-\alpha)$ e $X=X_{2}$ com probabilidade $\alpha$, ou seja, $X \sim \operatorname{TCEV}\left(\mu_{1}, \sigma_{1}, \mu_{2}, \sigma_{2}, \alpha\right)$. Então

$$
\mathrm{E}\left(X^{n}\right)=(1-\alpha) \mathrm{E}\left(X_{1}^{n}\right)+\alpha \mathrm{E}\left(X_{2}^{n}\right)
$$

\footnotetext{
${ }^{2}$ Para efeito de estimação dos parâmetros, seguimos a abordagem de Aitkin \& Rubin (1985), que sugerem impor restrições para os parâmetros mas não impor tais restrições para a estimação.
} 


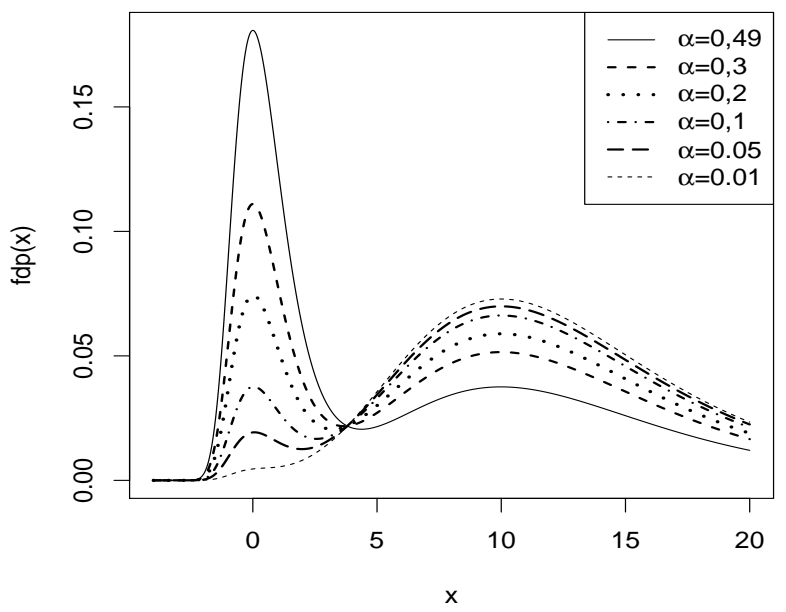

Figura 4.14: Função densidade de probabilidade da distribição $\operatorname{TCEV}(0,1,10,5, \alpha)$.

Podemos escrever

$$
\mathrm{E}(X)=(1-\alpha) \mathrm{E}\left(X_{1}\right)+\alpha \mathrm{E}\left(X_{2}\right)
$$

$\mathrm{e}$

$$
\operatorname{var}(X)=(1-\alpha) \operatorname{var}\left(X_{1}\right)+\alpha(1-\alpha)\left(\mathrm{E}\left(X_{1}\right)-\mathrm{E}\left(X_{2}\right)\right)^{2}+\alpha \operatorname{var}\left(X_{2}\right)
$$

em que a esperança e a variância de uma distribuição Gumbel são dadas, respectivamente, em (4.3) e (4.4).

O quantil $x_{p}$ não pode ser obtido analiticamente. Para estimar os quantis pode-se gerar uma amostra suficientemente grande e obter os quantis amostrais. Seja $Z \sim \operatorname{Bernoulli}(\alpha)$ e $X$ uma variável aleatória tal que

$$
X=\left\{\begin{array}{lll}
X_{1}, & \text { se } & Z=0 \\
X_{2}, & \text { se } & Z=1
\end{array}\right.
$$

então,

$$
\begin{aligned}
F_{X}(X \leq x) & =P(X \leq x)=P\left(X \leq x \mid X=X_{1}\right) P\left(X=X_{1}\right)+P\left(X \leq x \mid X=X_{2}\right) P\left(X=X_{2}\right) \\
& =P\left(X_{1} \leq x\right) P(Z=0)+P\left(X_{2} \leq x\right) P(Z=1) \\
& =(1-\alpha) \exp \left(-\exp \left(-\frac{x-\mu_{1}}{\sigma_{1}}\right)\right)+\alpha \exp \left(-\exp \left(-\frac{x-\mu_{2}}{\sigma_{2}}\right)\right) \\
& =F_{T C E V}\left(x ; \mu_{1}, \sigma_{1}, \mu_{2}, \sigma_{2}, \alpha\right) .
\end{aligned}
$$

Logo, para gerar uma observação de uma variável aleatória $X \sim \operatorname{TCEV}\left(\mu_{1}, \sigma_{1}, \mu_{2}, \sigma_{2}, \alpha\right)$ pode-se gerar uma observação de uma variável $Z \sim \operatorname{Bernoulli}(\alpha)$, e se $Z=0$ gerar uma observação $X_{1} \sim E V\left(\mu_{1}, \sigma_{1}\right)$ e se $Z=1$ gerar uma observação $X_{2} \sim E V\left(\mu_{2}, \sigma_{2}\right)$.

Os coeficientes de assimetria e de curtose de $X \sim \operatorname{TCEV}\left(\mu_{1}, \sigma_{1}, \mu_{2}, \sigma_{2}, \alpha\right)$ são obtidos através dos momentos (4.5) e (4.10) e das equações (4.6) e (4.7). O mínimo e o máximo dos coeficientes de assi- 
metria e de curtose para este caso particular são, respectivamente, $0,675,5,824,3,697$ e 67,594 (ver Apêndice F.8).

A Figura 4.15 apresenta os gráficos dos coeficientes de assimetria e de curtose da mistura de dois componentes da distribuição valor extremo $\operatorname{TCEV}(0,1,10,5, \alpha)$. Podemos observar que a distribuição de mistura é flexível em relação a Gumbel.
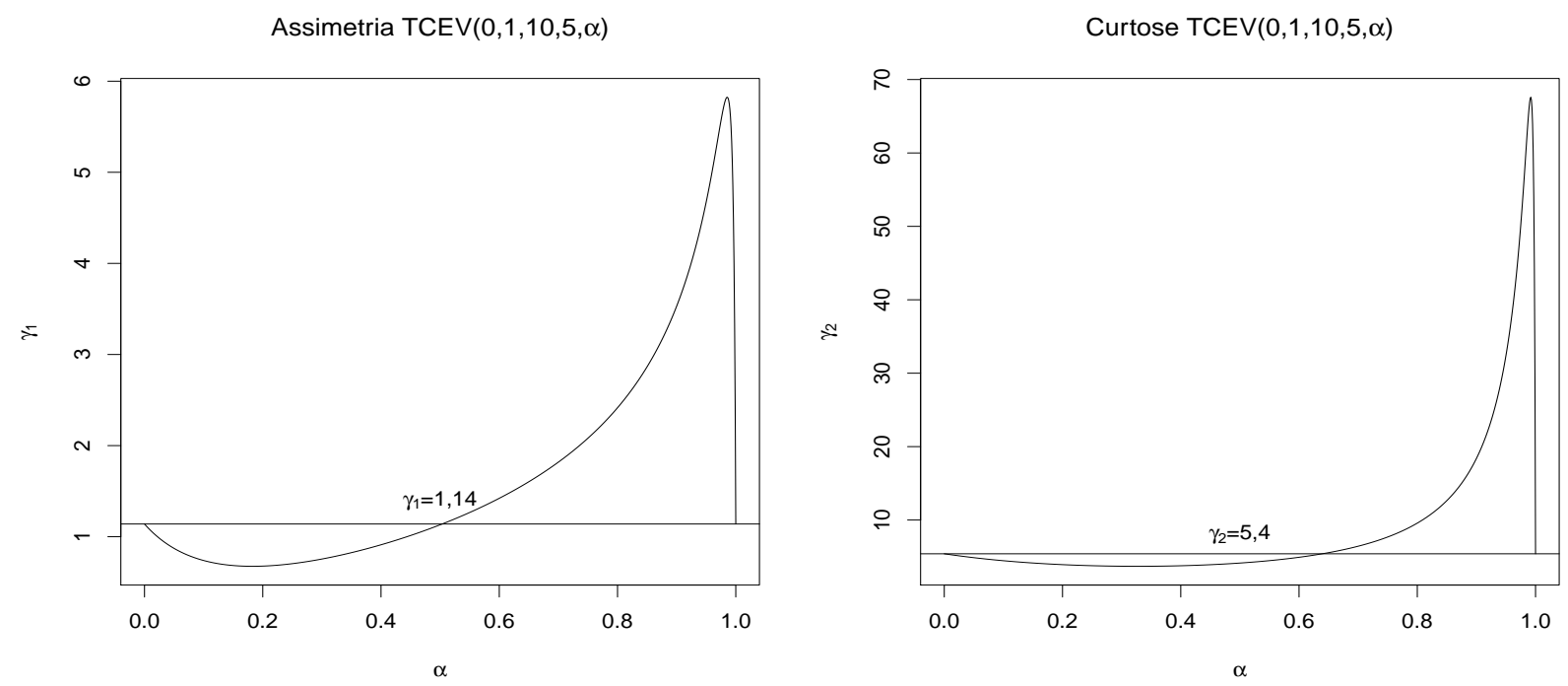

Figura 4.15: Gráficos dos coeficientes de assimetria e de curtose da mistura de dois componentes de distribuição Gumbel $T C E V(0,1,10,5, \alpha)$.

Observe que se definimos uma transmutação de classificação, cuja definição é apresentada na Seção 4.2.3, tal que

$$
T_{R}(u)=F_{2}\left(F_{1}^{-1}(u)\right)=\alpha u+(1-\alpha)\left[\exp \left(-[-\ln u]^{\sigma_{1} / \sigma_{2}}\right)\right]^{h\left(\mu_{1}, \mu_{2}, \sigma_{2}\right)}
$$

em que $h\left(\mu_{1}, \mu_{2}, \sigma_{2}\right)=\exp \left(\left(\mu_{2}-\mu_{1}\right) / \sigma_{2}\right)$ então

$$
F_{2}(x)=\alpha F_{1}(x)+(1-\alpha)\left[\exp \left(-\left[-\ln F_{1}(x)\right]^{\sigma_{1} / \sigma_{2}}\right)\right]^{\exp \left(\left(\mu_{2}-\mu_{1}\right) / \sigma_{2}\right)} .
$$

Se $F_{1}(x)=\exp \left(-\exp \left(-\left(x-\mu_{1}\right) / \sigma_{1}\right)\right)$ é a função de distribuição de uma variável aleatória com distribuição $E V\left(\mu_{1}, \sigma_{1}\right)$ então

$F_{2}(x)=\alpha \exp \left(-\exp \left(-\frac{x-\mu_{1}}{\sigma_{1}}\right)\right)+(1-\alpha) \exp \left(-\exp \left(-\frac{x-\mu_{2}}{\sigma_{2}}\right)\right)=F_{T C E V}\left(x ; \mu_{1}, \sigma_{1}, \mu_{2}, \sigma_{2}, \alpha\right)$

e podemos dizer que esta mistura de duas distribuições valor extremo máximo é um caso particular de transmutação de classificação proposta em Shaw \& Buckley (2007).

A distribuição TEV é um caso particular desta mistura se $-1<\alpha<-0,5$ e $\operatorname{TEV}(\mu, \sigma, \alpha)=$ $T C E V\left(\mu^{*}, \sigma, \mu, \sigma, 1+\alpha\right)$, em que $\mu^{*}=\mu+\sigma \ln 2$. E ainda se $-0,5 \leq \alpha<0$, e $T E V(\mu, \sigma, \alpha)=$ $\operatorname{TCEV}\left(\mu, \sigma, \mu^{*}, \sigma,-\alpha\right)$. Se $0<\alpha \leq 1$ a distribuição $\operatorname{TEV}(\mu, \sigma, \alpha)$ não é um caso particular da mistura 
$T C E V$ pois neste caso o coeficiente de ponderação da segunda distribuição $E V$ é negativo.

A Tabela 4.1 apresenta o diagrama das distribuições que têm como caso particular ou limite a distribuição Gumbel (EV) e os respectivos valores dos parâmetros para os quais as generalizações coicidem com os casos particulares. As distribuições com moldura quadrada são as que têm funções de distribuição acumulada não inversíveis. As distribuições com moldura redonda pontilhada não são identificáveis.

Tabela 4.1: Diagrama das generalizações da distribuição Gumbel

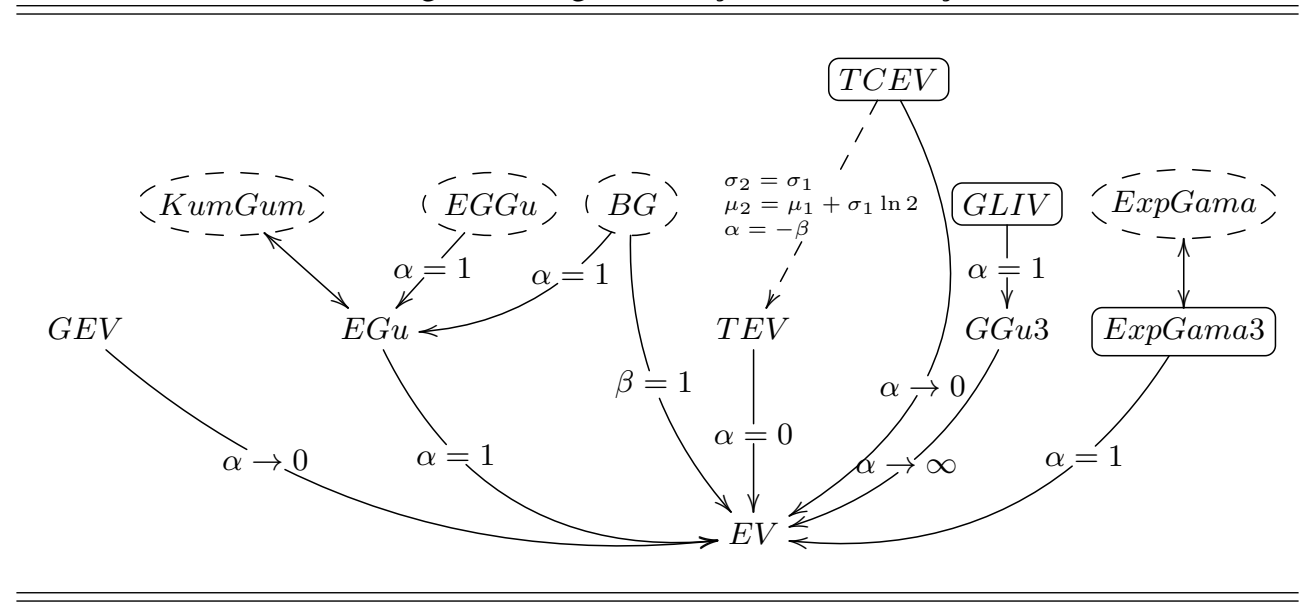

\subsection{Indice da cauda}

Numa linguagem coloquial, pode-se dizer que uma função de variação regular se comporta assintoticamente como uma função potência. Formalmente, uma função mensurável $U: \mathbb{R}^{+} \rightarrow \mathbb{R}^{+}$é de variação regular no infinito com índice $\alpha$, ou $U \in R V_{\alpha}$ se, para $x>0, \lim _{t \rightarrow \infty} U(t x) / U(t)=x^{\alpha}$. O índice $\alpha$ é denominado expoente de variação ou índice de variação regular. $\operatorname{Se} \alpha=0$, diz-se que $U$ é de variação lenta. A função $U$ é de variação lenta se e somente se $\lim _{t \rightarrow \infty} U(t x) / U(t)=1, \forall x \geq 1$. Se $U$ é monótona, é dita de variação lenta se e somente se, $\exists x>0, x \neq 1$ tal que $\lim _{t \rightarrow \infty} U(t x) / U(t)=1$. A função $U$ é dita de variação rápida ou de variação regular com índice infinito, ou $U \in R V_{\infty}$ (ver de Haan, 1970, p. 4), se $\forall x$

$$
\lim _{t \rightarrow \infty} \frac{U(t x)}{U(t)}:=x^{\infty}=\left\{\begin{array}{ccc}
0 & \text { se } & 0<x<1 \\
1 & \text { se } & x=1 \\
\infty & \text { se } & x>1
\end{array}\right.
$$

Analogamente, $U \in R V_{-\infty}$ se

$$
\lim _{t \rightarrow \infty} \frac{U(t x)}{U(t)}:=x^{-\infty}=\left\{\begin{array}{ccc}
\infty & \text { se } & 0<x<1 \\
1 & \text { se } & x=1 \\
0 & \text { se } & x>1
\end{array}\right.
$$

A função de variação canônica com índice $\alpha$ é $x^{\alpha}$. As funções $\ln (1+x)$ e $\ln \ln (e+x)$ são de variação lenta, assim como $\exp \left((\ln x)^{\alpha}\right), 0<\alpha<1$. Toda função $U$ tal que $\lim _{x \rightarrow \infty} U(x)$ existe, é positivo e finito, é de variação regular. As funções $e^{x}$ e $\sin (x+2)$ não são de variação regular (ver Resnick, 2007, p. 21). 
Uma distribuição $F$ é dita ter cauda direita pesada se a cauda direita, $\bar{F}:=1-F$, é uma função de variação regular com índice de variação regular negativo $\alpha=-1 / \xi$, isto é, $\lim _{t \rightarrow \infty} \bar{F}(t x) / \bar{F}(t)=x^{-1 / \xi}$. Toda distribuição $F$ de cauda direita pesada pertence ao domínio de atração de uma distribuição GEV com $\xi>0$, também chamada de família Fréchet, e escreve-se $F \in \mathcal{D}_{\mathcal{M}}\left(G E V_{\xi>0}\right)$ (Gnedenko, 1943; ver também Capítulo 1). O parâmetro $\xi$, um dos parâmetros fundamentais para eventos raros, é denominado índice da cauda.

Modelos com caudas pesadas têm sido usados para modelar vários fenômenos em economia, ecologia, bibliometria e biometria, entre outros. Ver, por exemplo, Markovich (2007) e Resnick (2007).

A distribuição valor extremo generalizada (GEV) é de variação regular com índice de variação $-1 / \xi$ e índice da cauda $\xi$ quando $\xi>0$ (família Fréchet). Outras distribuições que são de variação regular são apresentadas na Tabela 4.2. A distribuição valor extremo generalizada (GEV) é de variação rápida,

Tabela 4.2: Índice da cauda de distribuições

\begin{tabular}{c|ccccc}
\hline \hline & & & & & \\
distribuição & $\mathrm{t}-\operatorname{Student}(\nu)$ & Cauchy & $\mathrm{F}(\alpha, \beta)$ & $\operatorname{Burr}(\alpha, \beta)$ & $\log$-logística $(\alpha, \beta)$ \\
\hline índice da cauda & $1 / \nu$ & 1 & $2 / \beta$ & $1 /(\alpha \beta)$ & $1 / \beta$ \\
\hline \hline
\end{tabular}

ou seja, de variação regular com índice de variação infinito, quando $\xi=0$ (família Gumbel). As outras distribuições aqui tratadas, a saber, Gumbel exponencializada (EGu), valor extremo transmutado (TEV), Gumbel generalizada com três parâmetros (GGu3), exponencial-gama com três parâmetros (ExpGama3), logística generalizada tipo IV (GLIV) e mistura de dois componentes da distribuição valor extremo máximo (TCEV) são todas de variação rápida (ver Apêndice G). Portanto, de todas as distribuições estudadas aqui a distribuição GEV é a única que permite cauda direita mais pesada que a distribuição Gumbel.

\subsection{Medidas de qualidade de ajuste}

Apresentamos a seguir algumas medidas de qualidade de ajuste que serão usadas posteriormente.

Sejam $x_{1}, x_{2}, \ldots, x_{n}$ uma amostra aleatória observada de uma variável aleatória com função de distribuição acumulada $F(x)$ e seja $k_{x}$ o número de observações $x_{i} \leq x$. A função de distribuição acumulada empírica é a função escada $\widehat{F}(x)=k_{x} / n$. A estatística de Kolmogorov-Smirnov é definida como o máximo da diferença $|\widehat{F}(x)-F(x)|$, ou seja,

$$
K S=\max _{x}|\widehat{F}(x)-F(x)|
$$

Kolmogorov (1933),Smirnov (1939).

Segundo Labeyrie (1991) a estatística de Kolmogorov-Smirnov não é muito informativa para estudar a bondade do ajuste da cauda direita da distribuição contemplada então propõe a seguinte distância média ponderada:

$$
\int_{-\infty}^{\infty}|\widehat{F}(x)-F(x)| W(F(x)) d x
$$

em que $W(x)$ é uma função de ponderação. Considerando $W(x)=1 /(1-x)$, a distância média ponderada 
é estimada por

$$
L=\frac{1}{n} \sum_{i=1}^{n} \frac{\left|\widehat{F}\left(x_{i}\right)-F\left(x_{i}\right)\right|}{1-F\left(x_{i}\right)} .
$$

Anderson \& Darling (1952) consideraram uma medida de discrepância ou "distância" entre duas distribuições, de acordo com a noção usual de uma medida em um espaço de funções, a saber,

$$
n \int_{-\infty}^{\infty}[\widehat{F}(x)-F(x)]^{2} \psi[F(x)] d F(x) \equiv W_{n}^{2}
$$

em que $\widehat{F}(x)$ é a função de distribuição acumulada empírica e $\psi(t)(\geq 0)$ é alguma função previamente definida. Considerando $\psi(t)=1 /[t(1-t)], \psi(t)=1 /(1-t)$ e $\psi(t)=1 /(1-t)^{2}, W_{n}^{2}$ resulta em

$$
\begin{gathered}
A D=n \int_{-\infty}^{\infty} \frac{[\widehat{F}(x)-F(x)]^{2}}{F(x)[1-F(x)]} d F(x), \\
A D R=n \int_{-\infty}^{\infty} \frac{[\widehat{F}(x)-F(x)]^{2}}{[1-F(x)]} d F(x)
\end{gathered}
$$

$\mathrm{e}$

$$
A D 2 R=n \int_{-\infty}^{\infty} \frac{[\widehat{F}(x)-F(x)]^{2}}{[1-F(x)]^{2}} d F(x),
$$

respectivamente. A medida de qualidade de ajuste $A D R$ é preferível quando se deseja realçar a falta de ajuste na cauda direita; $A D 2 R$ acentua ainda mais a possível falta de ajuste nesta cauda.

Como $\widehat{F}(x)$ é uma função escada com saltos nas estatísticas de ordem, as estatísticas de AndersonDarling podem ser escritas em formas alternativas mais úteis para propósitos computacionais, dadas por

$$
\begin{gathered}
A D=-n-\frac{1}{n} \sum_{i=1}^{n}(2 i-1)\left[\ln z_{i}+\ln \left(1-z_{n+1-i}\right)\right], \\
A D R=\frac{n}{2}-2 \sum_{i=1}^{n} z_{i}-\frac{1}{n} \sum_{i=1}^{n}(2 i-1) \ln \left(1-z_{n+1-i}\right), \\
A D 2 R=2 \sum_{i=1}^{n} \ln \left(1-z_{i}\right)+\frac{1}{n} \sum_{i=1}^{n} \frac{(2 i-1)}{\left(1-z_{n+1-i}\right)},
\end{gathered}
$$

em que $z_{i}=F\left(x_{i: n}\right)$ e $x_{i: n}$ é a $i$-ésima estatística de ordem (Luceño, 2005, p. 906 e Apêndice B).

Castillo et al. (2005, p. 215 eq. (9.64)) propõem uma medida para avaliar a bondade do ajuste, a qual denomina de average scaled absolute error,

$$
A S A E=\frac{1}{n} \sum_{i=1}^{n} \frac{\left|x_{i: n}-\hat{x}_{i: n}\right|}{x_{n: n}-x_{1: n}},
$$

em que $x_{1: n} \leq x_{2: n} \leq \ldots \leq x_{n: n}$ são as estatísticas de ordem de uma amostra aleatória de uma distribuição $F(x), \hat{x}_{i: n}=F^{-1}\left(p_{i: n}\right) \operatorname{com} p_{i: n}=(i-0,5) / n$. 
Em teoria da probabilidade e teoria da informação, a divergência de Kullback-Leiber, também chamada de informação de divergência, ganho de informação, entropia relativa ou KLIC, é uma medida não simétrica da diferença entre duas distribuições de probabilidade. É uma medida da informação perdida quando uma distribuição é usada para aproximar a outra. Sejam $x_{1}, x_{2}, \ldots, x_{n}$ observações independentes de uma variável aleatória com função densidade de probabilidade $g(x)$. Seja $f(x \mid \theta)$ a função densidade de probabilidade de uma família paramétrica com vetor de parâmetros $\theta$. A informação média de Kullback-Lieber (KL) para discriminação entre $g(x)$ e $f(x \mid \theta)$ é dada por

$$
K L=\int \ln (g(x)) g(x) d x-\int \ln (f(x \mid \theta)) g(x) d x
$$

(ver Kullback, 1959, p. 6). Akaike $(1973,1974)$ deduziu que o logaritmo da verossimilhança calculado no estimador de máxima verossimilhança é um estimador enviesado da informação média de KullbackLieber (KL) e que este viés é aproximadamente igual ao número de parâmetros do modelo, isto é, $\ln \left(\prod_{i=1}^{n} f\left(x_{i} ; \hat{\theta}\right)\right)-k \approx K L$. Com base nisto, Akaike $(1973,1974)$ definiu o critério de informação, usualmente denominado AIC, dado por

$$
A I C=-2\left(\ln \left(\prod_{i=1}^{n} f\left(x_{i} ; \hat{\theta}\right)\right)-k\right) .
$$

Um modelo com valor menor de AIC é considerado um melhor modelo.

Quanto mais parâmetros tem um modelo maior é o logaritmo da verossimilhança calculado nos estimadores de máxima verossimilhança. Como o critério de Akaike é uma medida que inclui o logaritmo da verossimilhança calculado nos estimadores de máxima verossimilhança e o número de parâmetros do modelo, é uma medida mais adequada para comparar modelos com número de parâmetros diferentes do que simplesmente o valor do o logaritmo da verossimilhança calculado nos estimadores de máxima verossimilhança para cada um dos modelos.

O princípio da parcimônia propõe um equilíbrio entre o viés e a variância do estimador devidos ao número de parâmetros do modelo. Um modelo com poucos parâmetros relativamente ao melhor modelo terá provavelmente viés nas estimativas dos parâmetros, mas os parâmetros serão estimados com alta precisão. Este extremo não é desejável porque dá uma falsa ideia de precisão quando alguns parâmetros são bastante enviesados. Alternativamente, um modelo com muitos parâmetros também não é desejável, pois enquanto o viés é esperado ser pequeno, a precisão dos parâmetros estimados pode ser baixa ("estimativas instáveis"). Parcimônia visa obter um equilíbrio entre estes dois extremos. O critério de Akaike contempla a parcimônia já que leva em conta o número de parâmetros do modelo.

Quando o número de parâmetros $k$ é grande em relação ao tamanho da amostra $n$ (o que inclui o caso em que $n$ é pequeno, para qualquer valor de $k$ ), há uma correção de viés de segunda ordem para o critério AIC, denominada $C A I C$,

$$
C A I C=A I C+\frac{2 k(k+1)}{n-k-1}
$$

(ver Hurvich \& Tsai, 1989, 1995). O critério $C A I C$ converge para $A I C$ quando $n \rightarrow \infty$. Quando $n / k$ é 
maior que 40, para o modelo com o maior valor de $k$, o viés é pequeno e a correção é dispensável pois os valores de $A I C$ e $C A I C$ serão muito próximos. Na prática pode-se eleger simplesmente o modelo com menor valor de $C A I C$ que será correto em qualquer situação.

Schwarz (1978) definiu um critério, denominado BIC, para seleção de um modelo entre modelos de dimensões diferentes usando a teoria de Bayes. Deve-se eleger o modelo com menor valor de

$$
B I C=-2\left(\ln \left(\prod_{i=1}^{n} f\left(x_{i} ; \hat{\theta}\right)\right)+k \ln n\right) .
$$

$\mathrm{O}$ critério de seleção de um modelo entre modelos de dimensões diferentes $B I C$ é definido a partir dos termos da expansão assintótica do solução de Bayes. A solução de Bayes consiste na seleção do modelo que tem a posteriori mais provável. A posteriori mais provável é obtida maximizando-se a fórmula de Bayes definida a partir de uma função de perda pela escolha do modelo incorreto. O termo principal da expansão da fórmula de Bayes converge para o logaritmo da verossimilhança quando o tamanho da amostra cresce então o estimador de Bayes coincide com o estimador de máxima verossimilhança. Este termo principal depende apenas do suporte da priori e por isso este critério é válido para grandes amostras independentemente da priori que se considere. Qualitativamente $A I C$ e $B I C$ são uma formulação matemática do princípio da parcimônia na seleção de um modelo. Quantitativamente $B I C$ favorece um modelo com menos parâmetros (quando $n \geq 8$ ) comparativamente ao $A I C$. Para amostras muito grandes os dois critérios diferem significativamente. Segundo Schwarz (1978), sob as condições estabelecidas para definição do critério BIC, o critério de Akaike não é assintóticamente ótimo.

Um gráfico qqplot é um método gráfico de comparar um conjunto de dados $x_{1}, \ldots, x_{n}$ com uma distribuição de referência $G$. Se $F$ é a verdadeira distribuição (desconhecida) dos dados, a questão de interesse é se $F=G$, e se não, quanto elas diferem. Em um gráfico qqplot as estatísticas de ordem dos dados são plotadas contra os valores esperados das estatísticas de ordem de uma amostra de $G$, ou contra os quantis aproximados dos valores esperados. Desvios da linha de 45 graus sugerem a amplitude e natureza da diferença entre $F$ e $G$. Uma interpretação apropriada de um gráfico qqplot requer informação sobre a variabilidade amostral. Um método simples de obter esta informação é através de um gráfico qqplot com envelope, descrito pela primeira vez em Ripley (1977). Uma descrição detalhada da construção de um gráfico qqplot com envelope pode ser vista em Atkinson (1985). Para construir um envelope pode-se usar a função envelope do pacote boot do software R (R Development Core Team, 2013). A presença de uma quantidade significativa de pontos fora do envelope simulado é evidência contra as observações terem sido amostradas de $G$.

\subsection{Aplicações}

Apresentamos a seguir a aplicação do disposto acima a conjuntos de dados.

Para análise descritiva usamos o gráfico de dispersão e o boxplot ajustado para distribuições assimétricas definido em Hubert \& Vandervieren (2008). Para a produção do gráfico boxplot ajustado usamos a função adjbox do pacote robustbase do software R.

Para comparar a qualidade do ajuste das distribuições estudadas usamos a estatística de Kolmogorov- 
Smirnov (4.11), os erros ponderados sugeridos por Labeyrie (1991) (4.12), as estatísticas de AndersonDarling $A D$ (4.13), $A D R$ (4.14) e $A D 2 R$ (4.15), o critério ASAE de Castillo et al. (2005, p. 215 eq. (9.64)) (4.16), o critério de Akaike AIC (4.17), que considera o número de parâmetros do modelo, o critério de Akaike consistente CAIC (4.18), o critério BIC (4.19) e gráficos qqplot com envelope simulado.

Uma medida que é de interesse em modelagem de dados de valores extremos é o nível de retorno. O quantil $x_{1-p}$, tal que $F\left(x_{1-p}\right)=1-p$, é chamado de nível de retorno associado ao período $1 / p$. Espera-se que o nível $x_{1-p}$ seja excedido em média uma vez em $1 / p$ períodos, em que período é o tempo dos blocos em que se tomam o valor máximo. Geralmente o tempo do bloco é de um ano, isto é, os dados originais são divididos em blocos de um ano, e considera-se que o conjunto dos máximos dos blocos tem uma determinada distribuição. Assim, por exemplo, se $p=0,01$ espera-se que o nível $x_{1-p}$ seja excedido em média uma vez a cada 100 anos.

As estimativas dos parâmetros e os respectivos erros padrão foram calculados com a função de maximização MaxSQP do software OX que utiliza uma técnica de programação quadrática sequencial similar ao Algoritmo 18.7 de Nocedal \& Wright (1999). Esta função permite maximizar função não linear condicionada a restrição dos parâmetros e gera estimativas confiáveis da matriz Hessiana. Vale ressaltar que em todas as avaliações numéricas foram utilizadas derivadas numéricas.

A Tabela 4.3 apresenta os valores dos parâmetros adicionais para os quais as generalizações apresentadas na Seção 4.2 se reduzem à distribuição Gumbel como caso particular ou limite, seus respectivos índices de variação regular, índices da cauda e mínimos e máximos de assimetria e curtose.

Tabela 4.3: Características das generalizações da distribuição Gumbel

\begin{tabular}{lcccccc}
\hline \hline & $\alpha$ & $\beta$ & $\begin{array}{c}\text { índice de } \\
\text { variação regular }\end{array}$ & $\begin{array}{c}\text { índice da } \\
\text { cauda }\end{array}$ & assimetria & curtose \\
\hline GEV $_{\alpha \geq 0}$ & 0 & - & $(-\infty, 0)$ & {$[0, \infty)$} & $(-\infty ; \infty)^{1}$ & $(2,711 ; \infty)^{2}$ \\
EGu & 1 & - & $-\infty$ & 0 & $(-0,692 ; 1,97)$ & $(3,023 ; 8,850)$ \\
TEV & 0 & - & $-\infty$ & 0 & $(0,718 ; 1,321)$ & $(4,052 ; 6,705)$ \\
GGu3 & $\infty$ & - & $-\infty$ & 0 & $(-2 ; 1,14)$ & $(5,4 ; 9)$ \\
ExpGama3 & 1 & - & $-\infty$ & 0 & $(0 ; 2)$ & $(3 ; 9)$ \\
GLIV & 1 & $\infty$ & $-\infty$ & 0 & $(-2 ; 2)$ & $(3 ; 9)$ \\
TCEV & 0 & - & $-\infty$ & 0 & $(0,675 ; 5,824)^{3}$ & $(3,697 ; 67,594)^{3}$ \\
\hline \hline
\end{tabular}

${ }^{1}$ Para $\alpha<1 / 3 . \quad{ }^{2}$ Para $\alpha<1 / 4 . \quad{ }^{3} \operatorname{Para}\left(\mu, \sigma, \mu^{*} \sigma^{*}\right)=(0,1,10,5)$.

\subsubsection{Amostra simulada de distribuição Gumbel}

A primeira aplicação considerada utiliza uma amostra aleatória gerada de uma distribuição Gumbel padrão , $E V(0,1)$, de tamanho $n=500$. A Figura 4.16 apresenta o gráfico de observações versus respectivos índices e o boxplot ajustado dos dados. Nota-se que a maior parte das observações são próximas de zero, um número menor de observações está em torno de 4 e duas observações estão destacadas das outras. Os coeficientes amostrais de assimetria e de curtose são 1,19 e 5,23, respectivamente, próximos dos coeficientes da distribuição Gumbel (1,14 e 5,4).

A Tabela 4.4 apresenta as estimativas de máxima verossimilhança dos parâmetros das distribuições estu- 

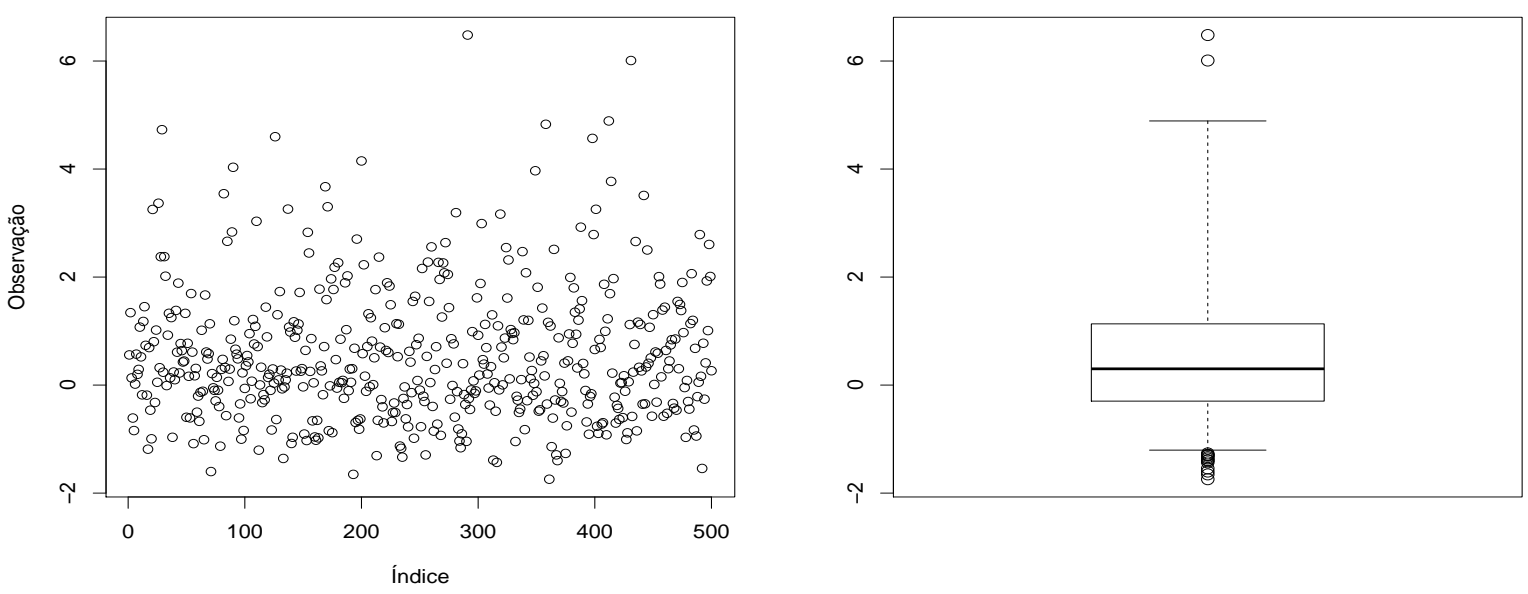

Figura 4.16: Gráfico de observação versus índice e boxplot ajustado de amostra simulada de distribuição $E V(0,1)$.

dadas (erros padrão entre parênteses) ${ }^{3}$, a estimativa do nível de retorno associado ao período de 100 anos e o logaritmo da verossimilhança calculado nas estimativas de máxima verossimilhança dos parâmetros $(\ell(\widehat{\theta}))$. Nota-se que, para as distribuições que têm a distribuição Gumbel como caso particular, as estimativas dos parâmetros junto com os erros padrão são compatíveis com uma distribuição $E V(0,1)$. Para a distribuição GGu3, que tem a distribuição Gumbel como caso limite quando $\alpha \rightarrow \infty$, a estimativa de $\alpha$ apresenta valor alto como esperado. Para a distribuição GLIV, que tem a distribuição Gumbel como caso limite quando $\alpha=1$ e $\beta \rightarrow \infty$, a estimativa de $\alpha$ é compatível com seu valor teórico e a estimativa de $\beta$ apresenta valor alto como esperado. As estimativas dos níveis de retorno de 100 anos para as diferentes distribuições são muito próximas. Os valores do logaritmo da verossimilhança maximizada são todos muito próximos. $\mathrm{O}$ maior valor é alcançado pela mistura de duas distribuições valor extremo máximo (TCEV), mas esta é a distribuição que tem o maior número de parâmetros e um número maior de parâmetros sempre aumenta o valor da verossimilhança. A Figura 4.17 apresenta o histograma com as densidades ajustadas. As curvas se

Tabela 4.4: Estimativas dos parâmetros, erros padrão entre parênteses e quantis; Amostra simulada de distribuição $E V(0,1)$

\begin{tabular}{lcccccrc}
\hline \hline & $\mu$ & $\sigma$ & $\alpha$ & $\beta$ ou $\mu^{*}$ & $\sigma^{*}$ & $x_{0,99}$ & $\ell(\widehat{\theta})$ \\
\hline EV & $-0,012(0,044)$ & $0,945(0,033)$ & - & - & - & 4,337 & $-762,976$ \\
GEV & $-0,021(0,047)$ & $0,940(0,034)$ & $0,017(0,032)$ & - & - & 4,480 & $-762,823$ \\
EGu & $-0,100(0,162)$ & $0,888(0,108)$ & $0,898(0,176)$ & - & - & 4,453 & $-762,825$ \\
TEV & $0,163(0,277)$ & $1,022(0,144)$ & $0,305(0,457)$ & - & - & 4,496 & $-762,796$ \\
GGu3 & $-0,012(0,049)$ & $0,945(0,035)$ & $6695,810(-)$ & - & - & 4,337 & $-762,977$ \\
ExpGama3 & $-0,154(0,245)$ & $0,875(0,126)$ & $0,881(0,201)$ & - & - & 4,462 & $-762,818$ \\
GLIV & $-0,043(0,035)$ & $0,864(0,071)$ & $0,869(0,118)$ & $170,755(-)$ & - & 4,459 & $-762,821$ \\
TCEV & $-0,032(0,068)$ & $0,922(0,061)$ & $0,012(0,038)$ & $3,301(3,895)$ & $0,983(0,704)$ & 4,514 & $-762,697$ \\
\hline \hline
\end{tabular}

vêem bem próximas.

\footnotetext{
${ }^{3}$ Os erros padrão dos parâmetros $\alpha$ da distribuição GGu3 e $\beta$ da distribuição GLIV não são apresentados pois não são confiáveis
} 


\section{Histograma de amostra simulada de $\operatorname{EV}(0,1)$}

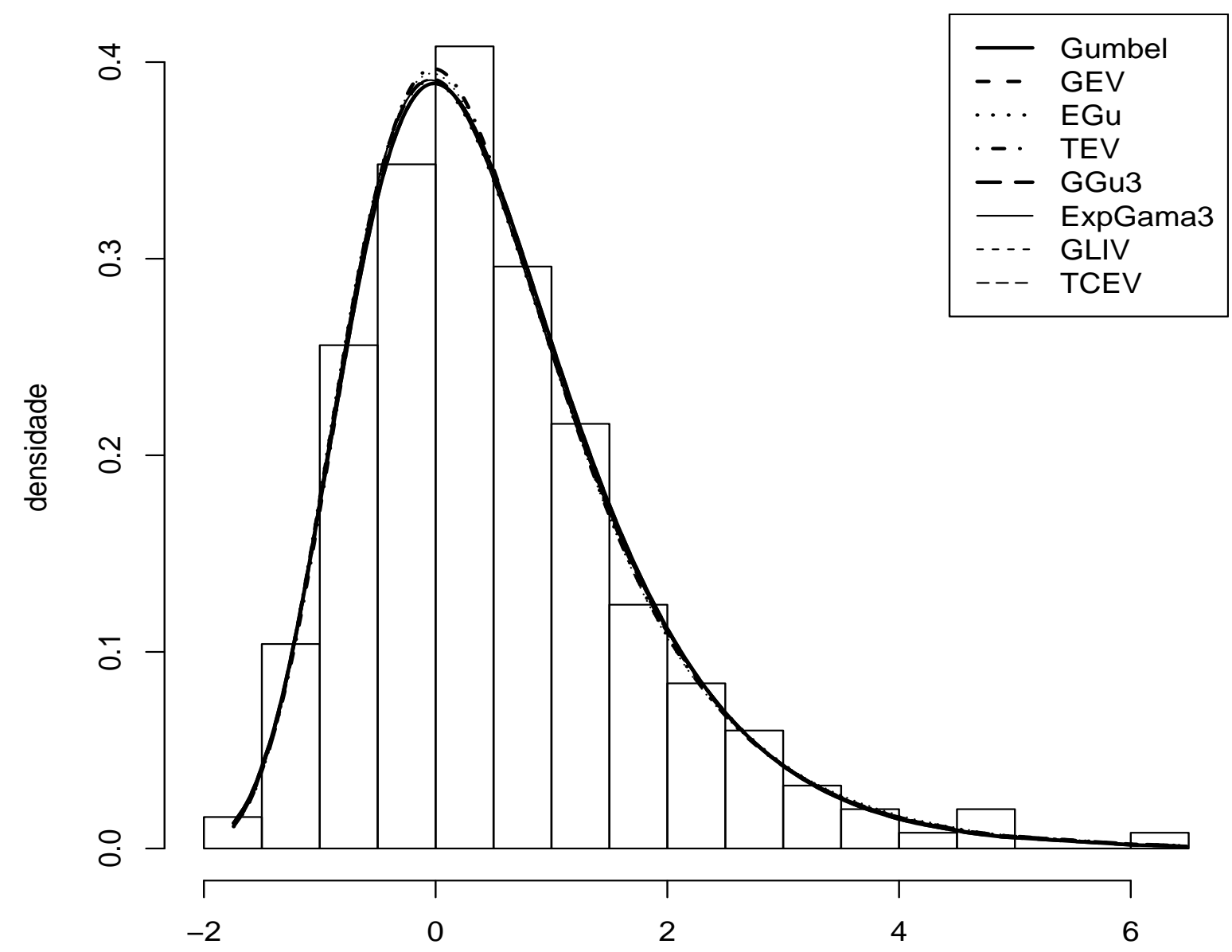

Figura 4.17: Histograma de amostra simulada de distribuição $E V(0,1)$ e densidades ajustadas.

A Tabela 4.5 apresenta o número de parâmetros $(k)$ e as medidas de bondade de ajuste. Note-se que valores menores das medidas sugerem melhores ajustes. A estatística de Kolmogorov-Smirnov (KS) não distingue as distribuições pois todas as distribuições apresentam a mesma distância da distribuição empírica segundo esta estatística. Quanto às outras medidas de qualidade de ajuste, média do erro de Labeyrie (L), estatística de Anderson-Darling (AD), estatística de Anderson-Darling com maior peso na cauda direita (ADR) e estatística de Anderson-Darling com peso quadrático na cauda direita (AD2R), as distribuições TEV e TCEV estão entre as melhores, no entanto as medidas das diferentes distribuições não se diferenciam muito entre si. Quanto aos critérios de informação, que penalizam a qualidade do ajuste pelo número de parâmetros, o menor valor de AIC é o da distribuição Gumbel $(1529,952)$. Dentre as distribuições com três parâmetros a que tem menor AIC é a distribuição TEV (1531,593). A distribuição TCEV apresenta o maior AIC, que é consequência do maior número de parâmetros desta distribuição. Os outros critérios de 
informação, CAIC e BIC, apresentam o mesmo comportamento que AIC. Observa-se que as medidas de bondade de ajuste da distribuição Gumbel não são muito diferentes das distribuições com mais parâmetros, o que é confirmado pelos valores de AIC e BIC que são similares para todas as distribuições.

Tabela 4.5: Bondade de ajuste; Amostra simulada de distribuição $E V(0,1)$

\begin{tabular}{lcccccccccc}
\hline \hline & $k$ & KS & L & AD & ADR & AD2R & ASAE & AIC & CAIC & BIC \\
\hline EV & 2 & 0,022 & 0,028 & 0,188 & 0,091 & 2,358 & 0,004 & 1529,952 & 1529,976 & 1538,381 \\
GEV & 3 & 0,022 & 0,020 & 0,177 & 0,069 & 1,463 & 0,003 & 1531,646 & 1531,694 & 1544,289 \\
EGu & 3 & 0,022 & 0,021 & 0,187 & 0,075 & 1,523 & 0,003 & 1531,651 & 1531,699 & 1544,294 \\
TEV & 3 & 0,020 & 0,018 & 0,152 & 0,056 & 1,401 & 0,003 & 1531,593 & 1531,641 & 1544,236 \\
GGu3 & 3 & 0,022 & 0,028 & 0,188 & 0,091 & 2,360 & 0,004 & 1531,954 & 1532,002 & 1544,598 \\
ExpGama3 & 3 & 0,022 & 0,021 & 0,185 & 0,073 & 1,500 & 0,003 & 1531,636 & 1531,685 & 1544,280 \\
GLIV & 4 & 0,022 & 0,019 & 0,174 & 0,066 & 1,472 & 0,003 & 1533,642 & 1533,723 & 1550,500 \\
TCEV & 5 & 0,022 & 0,018 & 0,163 & 0,059 & 1,337 & 0,003 & 1535,394 & 1535,516 & 1556,467 \\
\hline \hline
\end{tabular}

A Figura 4.18 apresenta os gráficos qqplot para cada uma das distribuições estudadas. Os gráficos não apresentam pontos fora do envelope, logo não há evidência contra as observações terem sido amostradas das respectivas distribuições, como é de se esperar uma vez que todas as distribuições têm como caso particular ou caso limite a distribuição Gumbel da qual os dados foram gerados.

A Figura 4.19 mostra os gráficos do logaritmo da verossimilhança perfilada para o(os) parâmetro(os) adicionais das diversas distribuições generalizadas. Para a distribuição TEV o gráfico do logaritmo da verossimilhança perfilada para $\alpha$ apresenta um ponto de inflexão, um ponto de mínimo local e dois pontos de máximo local, portanto, a convergência da otimização para um máximo local pode ocorrer dependendo da escolha do valor inicial para $\alpha$. Para a distribuição GGu3 o gráfico do logaritmo da verossimilhança perfilada para $\alpha$ apresenta crescimento lento para valores grandes de $\alpha$. $\mathrm{O}$ valor da estimativa, neste caso, é dependente da precisão usada no algoritmo de estimação. Observa-se que a estimativa de $\alpha$ para a distribuição GGu3 é 6695,810 e no entanto a função é crescente para este valor de $\alpha$. O mesmo ocorre com o gráfico da distribuição GLIV na direção do parâmetro $\beta$. Por questões numéricas, quando a estimativa do parâmetro é um valor grande e o logaritmo da verossimilhança perfilada para este parâmetro é uma função de crescimento lento, a estimação do erro padrão fica comprometida, como é o caso para o parâmetro $\alpha$ da distribuição GGu3 e o parâmetro $\beta$ da distribuição GLIV. Para a distribuição ExpGama3 o gráfico apresenta uma curva pouco côncava o que aumenta o erro padrão. Para a distribuição TCEV o gráfico apresenta uma curva não suave num intervalo de $\alpha$, provavelmente por questões numéricas, que não parece dificultar a otimização. Para a distribuição do valor extremo generalizada (GEV) e a distribuição Gumbel exponencializada (EGu) as curvas são mais regulares do que as outras generalizações aqui apresentadas. Elas não exibem nenhuma das características indesejáveis como ponto de inflexão, multimodalidade, crescimento lento e baixa concavidade. Uma consequência importante deste comportamento mais regular é que a estimação de máxima verossimilhança é um problema de otimização mais simples.

\subsubsection{Velocidade máxima mensal do vento em West Palm Beach de 1984-2012}

Nesta seção analisamos a qualidade do ajuste das generalizações da distribuição Gumbel aqui estudadas ao conjunto de dados de máximas mensais da velocidade do vento (mph - milhas /hora) com duração de 

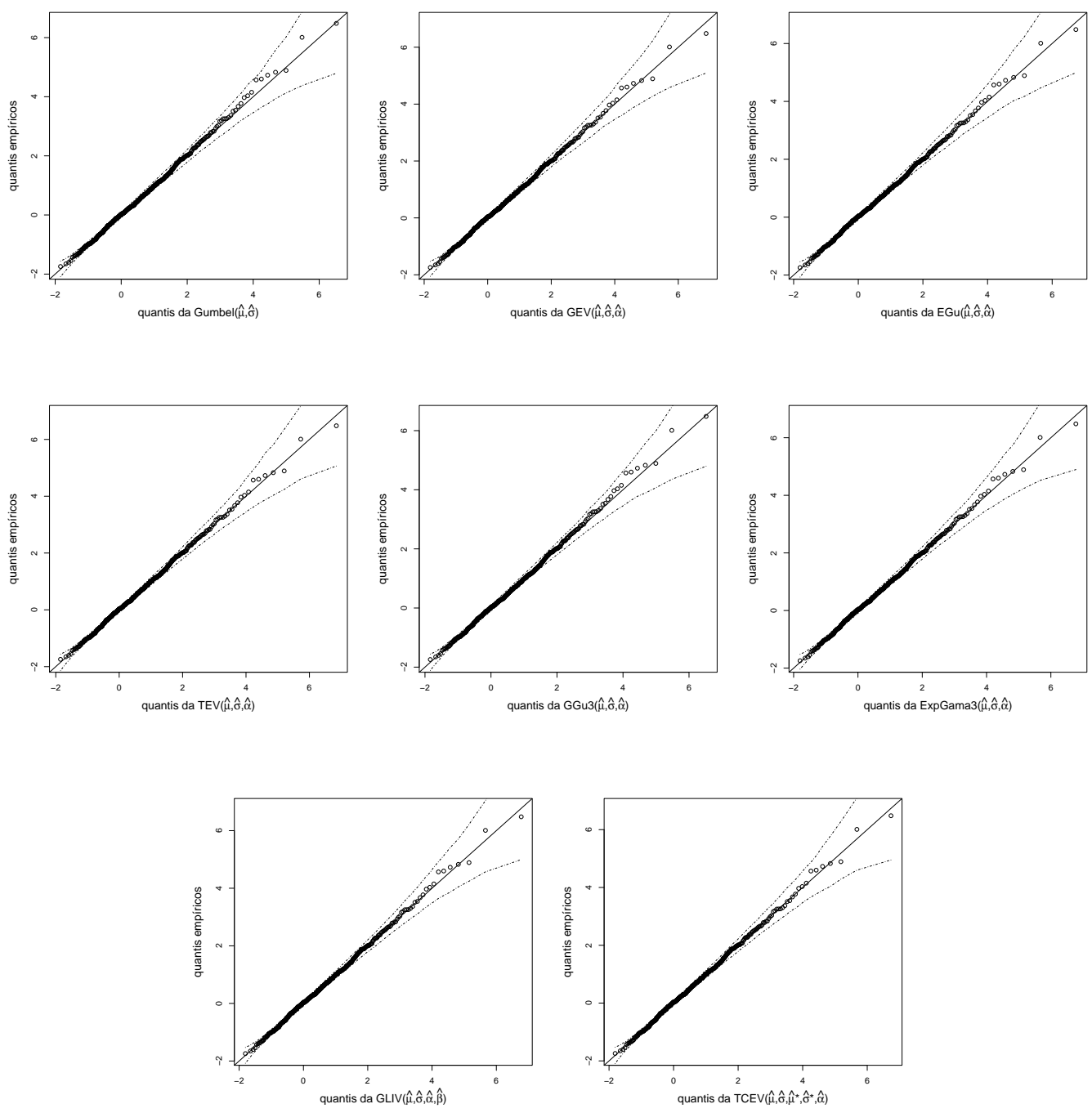

Figura 4.18: QQplots - amostra simulada de distribuição $E V(0,1)$,

5 segundos em West Palm Beach na Flórida de 1984 a 2012, com $n=348$ observações. Os dados foram obtidos em National Climate Data Center (NCDC) - National Oceanic and Atmospheric Administration (NOAA) no endereço http: //www.ncdc.noaa.gov/. 4

Apresentamos na Figura 4.20 o diagrama de dispersão e o boxplot ajustado dos dados. Nota-se que, ao contrário do boxplot ajustado para a amostra simulada de uma distribuição Gumbel, não há presença relevante de observações discrepantes na cauda esquerda e há mais observações discrepantes na cauda direita. Os coeficientes amostrais de assimetria e de curtose são 2,08 e 11,27, respectivamente, bem superiores aos da distribuição Gumbel (1,14 e 5,4), o que sugere o uso das distribuições generalizadas. Observe que as únicas distribuições cuja assimetria pode ser maior que 2 e cuja curtose pode ser maior que 9 são as distribuições GEV e TCEV.

\footnotetext{
${ }^{4}$ Mais especificamente, http://WwW.ncdc.noaa.gov/ $\rightarrow$ I want to search for data at a particular location. $\rightarrow$ Addittional Data Acess:Publications $\rightarrow$ Local Climatological Data.
} 

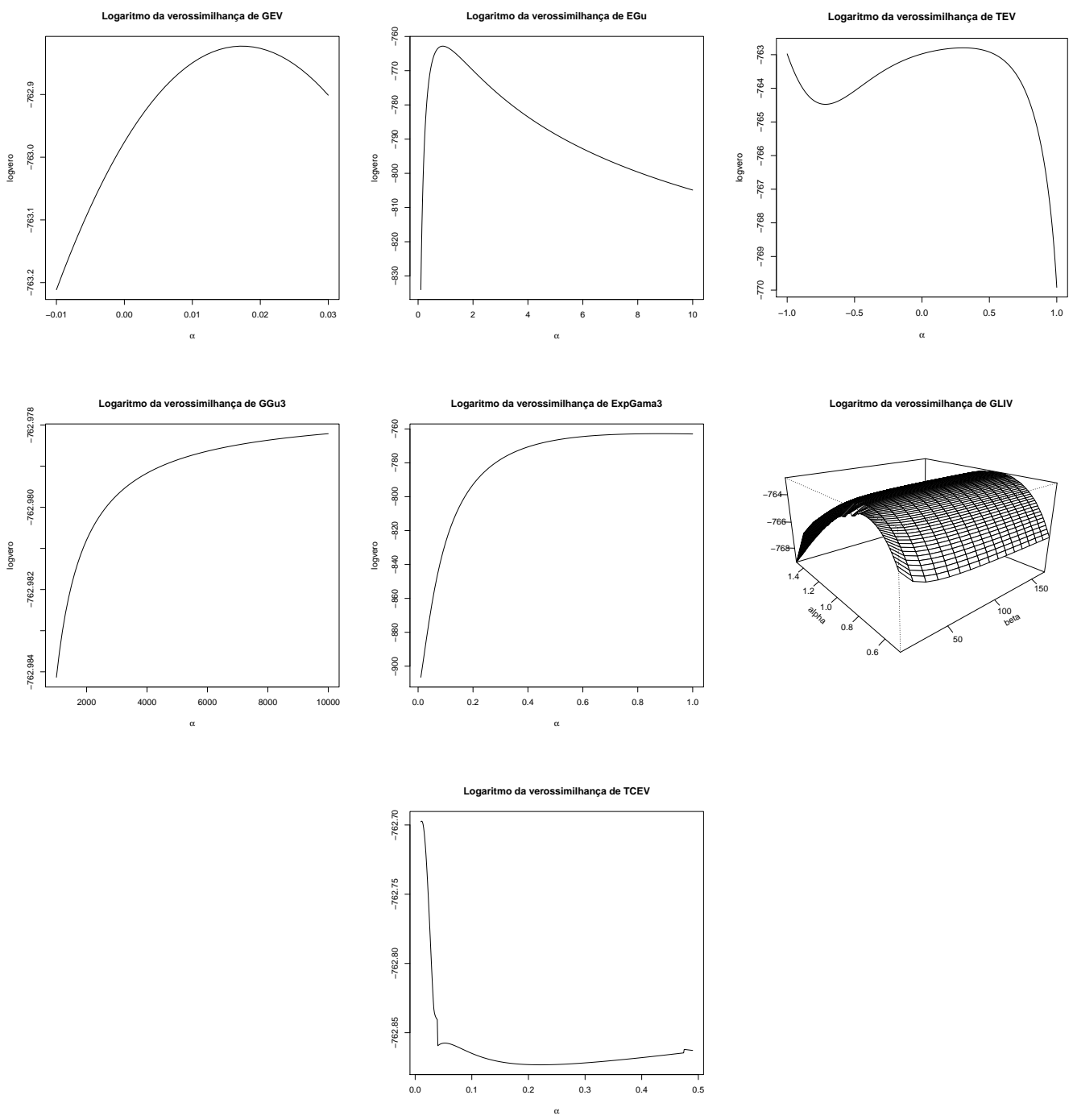

Figura 4.19: Logaritmo da verossimilhança perfilada - amostra simulada de distribuição $E V(0,1)$.

A Tabela 4.6 apresenta as estimativas de máxima verossimilhança dos parâmetros das distribuições estudadas (erros padrão entre parênteses), a estimativa do nível de retorno associado ao período de 100 anos e o logaritmo da verossimilhança calculado nas estimativas de máxima verossimilhança dos parâmetros $(\ell(\widehat{\theta})$ ). A estimativa do parâmetro adicional $\alpha$ para a distribuição GGu3 é muito alto, ou seja, a distribuição GGu3 ajustada praticamente coincide com a distribuição Gumbel. De fato, as estimativas de $\mu$ e $\sigma$ desta duas distribuições coincidem até a terceira casa decimal. Esta distribuição não apresentou maior flexibilidade do que a distribuição Gumbel para se ajustar a este conjunto de dados. Nota-se que estas duas distribuições produziram os menores valores do logaritmo da verossimilhança maximizada. O maior valor do logaritmo da verossimilhança é alcançado pela mistura de duas distribuições valor extremo máximo (TCEV) $(-1180,084)$ seguido pelo da distribuição valor extremo generalizada (GEV) $(-1180,898)$. Apesar da distribuição TCEV ter cinco parâmetros, o valor do logaritmo da verossimilhança está próximo do da distribuição GEV, que tem apenas três parâmetros. Os níveis de retorno associado ao perído de 100 anos para as diferentes distribuições 

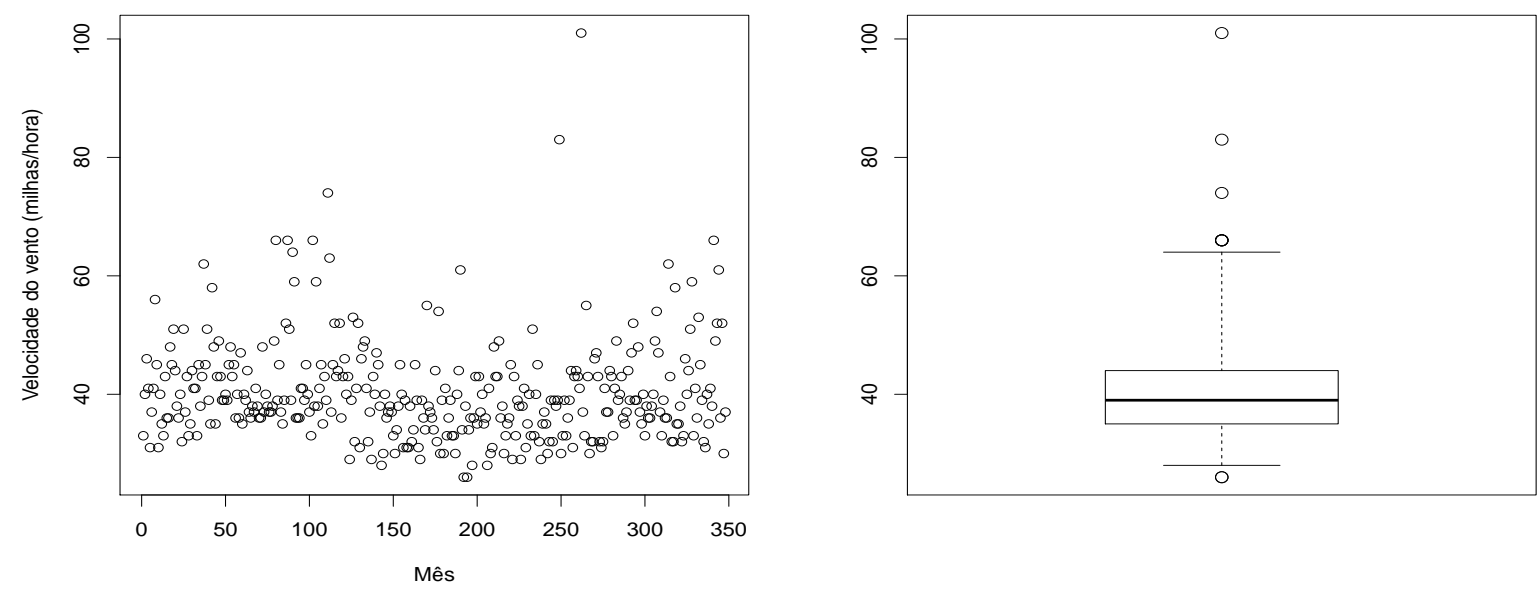

Figura 4.20: Diagrama de dispersão e boxplot ajustado da velocidade máxima mensal do vento (milhas/hora) em West Palm Beach/Flórida de 1984 a 2012.

que se espera ser excedido em média uma vez a cada 100 anos diferem em até cinco unidades, sendo o menor valor o da distribuição Gumbel $(64,546)$ e os maiores os da distribuição $\operatorname{GEV}(69,321)$ e TCEV $(71,877)$. A Figura 4.21 apresenta o histograma com as densidades ajustadas. As curvas são distinguíveis não se

Tabela 4.6: Estimativas dos parâmetros e erros padrão (entre parênteses); velocidade máxima mensal do vento - West Palm Beach - 1984 a 2012

\begin{tabular}{lccccccc}
\hline \hline & $\mu$ & $\sigma$ & $\alpha$ & $\beta$ ou $\mu^{*}$ & $\sigma^{*}$ & $x_{0,99}$ & $\ell(\widehat{\theta})$ \\
\hline EV & $36,667(0,341)$ & $6,060(0,258)$ & - & - & - & 64,546 & $-1184,071$ \\
GEV & $36,392(0,358)$ & $5,873(0,274)$ & $0,083(0,038)$ & - & - & 69,321 & $-1180,898$ \\
EGu & $34,665(0,926)$ & $4,697(0,634)$ & $0,657(0,134)$ & - & - & 67,589 & $-1182,093$ \\
TEV & $38,623(0,858)$ & $7,001(0,559)$ & $0,525(0,194)$ & - & - & 65,743 & $-1182,308$ \\
GGu3 & $36,667(0,297)$ & $6,060(0,225)$ & $11892,399(-)$ & - & - & 64,545 & $-1184,073$ \\
ExpGama3 & $33,757(1,115)$ & $4,480(0,669)$ & $0,622(0,137)$ & - & - & 67,719 & $-1182,007$ \\
GLIV & $35,923(0,527)$ & $3,599(1,195)$ & $0,488(0,190)$ & $5,767(9,540)$ & - & 68,014 & $-1181,760$ \\
TCEV & $36,275(0,393)$ & $5,551(0,335)$ & $0,032(0,028)$ & $59,756(12,048)$ & $10,262(5,104)$ & 71,877 & $-1180,084$ \\
\hline \hline
\end{tabular}

sobrepondo.

A Tabela 4.7 apresenta o número de parâmetros $(k)$ e as medidas de bondade de ajuste. A estatística de Kolmogorov-Smirnov (KS) com menor valor é a da distribuição TEV (0,057), seguida pelas distribuições Gumbel, GGu3, GLIV e TCEV (0,066). A média do erro de Labeyrie (L) atinge o menor valor para a distribuição TCEV (0,048), seguida por GEV $(0,061)$. A estatística de Anderson-Darling (AD) apresenta um comportamento parecido com a média do erro de Labeyrie, sendo a distribuição TCEV a que apresenta o menor valor $(0,801)$ seguida por TEV $(0,916)$ e GEV $(0,944)$. As distribuições Gumbel e GGu3 as que apresentam a maior distância (1,119). As estatísticas AD das outras distribuições são próximas entre si. A estatística de Anderson-Darling com maior peso na cauda direita (ADR) que apresenta o menor valor é a da distribuição TCEV (0,325), seguida por GEV (0,417). As distribuições Gumbel e GGu3 são as que apresentam o maior valor $(0,618)$. A estatística de Anderson-Darling com peso quadrático na cauda 


\section{Histograma de velocidade do vento em West Palm Beach}

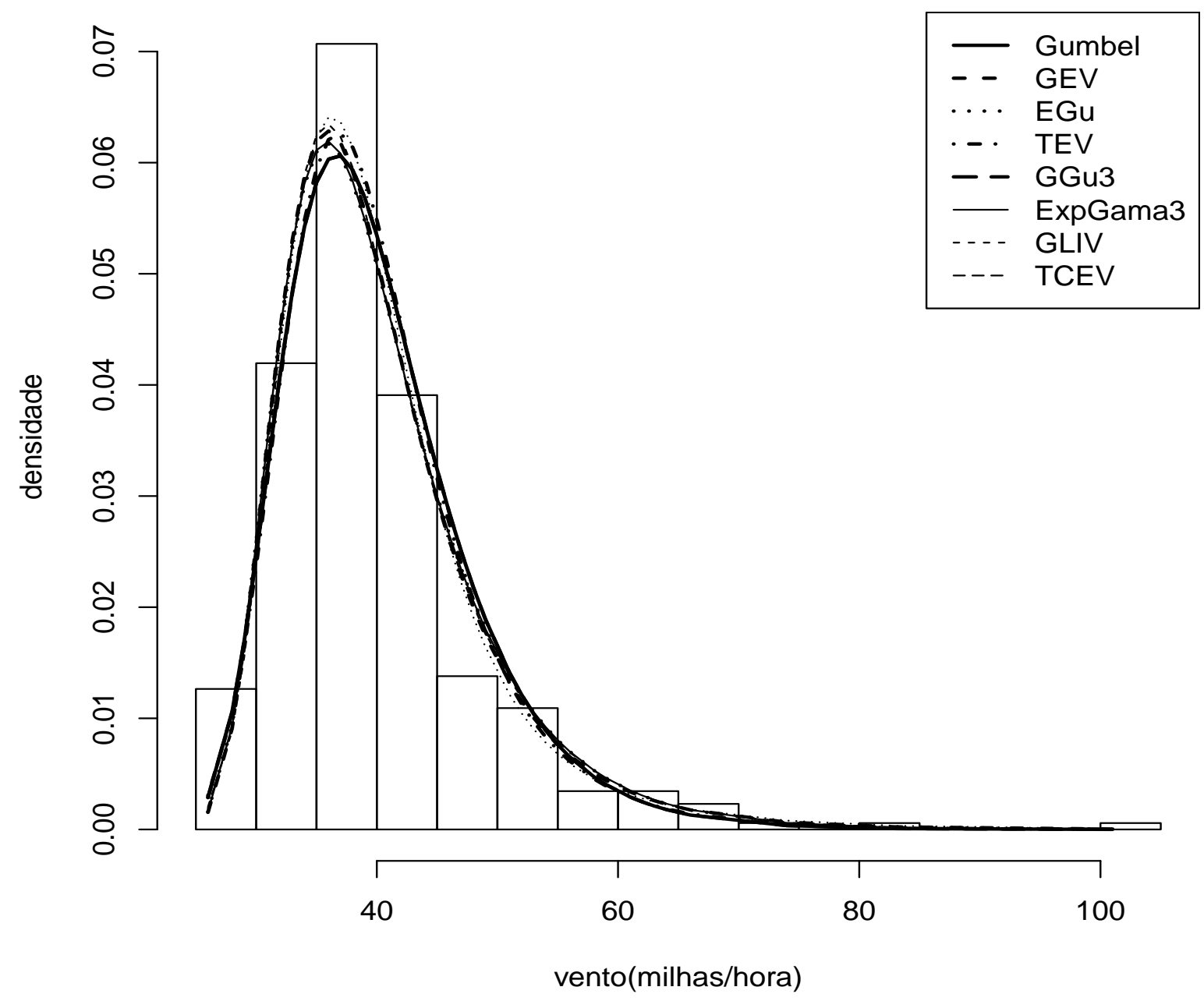

Figura 4.21: Histograma e densidades ajustadas da velocidade máxima mensal do vento (milhas/hora) em West Palm Beach na Flórida de 1984 a 2012.

direita (AD2R) destaca notoriamente de forma negativa a distribuição Gumbel (130,060) e GGu3 (130,112), enquanto indica como melhores distribuições a distribuição $\operatorname{TCEV}(3,351)$ seguida por GEV $(5,646)$. A estatística ASAE só se distingue entre as distribuições na terceira casa decimal, sendo os menores valores o das distribuições TCEV $(0,006)$ e GEV $(0,007)$. O menor AIC é o da distribuição GEV $(2367,795)$ seguido por ExpGama3 $(2370,013)$ e TCEV $(2370,168)$. O mesmo ocorre com CAIC, como esperado uma vez que a amostra não é pequena. O menor BIC é o da distribuição GEV $(2379,352)$ seguido pelo da distribuição Gumbel $(2379,847)$, evidenciando a influência do número de parâmetros neste critério. Observa-se que as medidas de bondade de ajuste da distribuição Gumbel revelam que esta não é a melhor opção para ajuste deste conjunto de dados, sendo as distribuições TCEV e GEV as que se destacaram na maioria dos critérios contemplados, apresentando os menores valores. Os resultados dos critérios de informação AIC e 
CAIC apresentam os maiores valores para a distribuição Gumbel sendo esta rejeitada como opção de melhor ajuste segundo estes critérios. A distribuição GEV tem os menores valores de AIC, CAIC e BIC entre as distribuições ajustadas. Considerando todos os critérios concluímos que a distribuição GEV é a que melhor se ajusta a este conjunto de dados.

Tabela 4.7: Bondade do ajuste; velocidade máxima mensal do vento - West Palm Beach - 1984 a 2012

\begin{tabular}{lccccccccc}
\hline \hline & KS & L & AD & ADR & AD2R & ASAE & AIC & CAIC & BIC \\
\hline EV & 0,066 & 0,277 & 1,119 & 0,618 & 130,060 & 0,009 & 2372,143 & 2372,177 & 2379,847 \\
GEV & 0,069 & 0,061 & 0,944 & 0,417 & 5,646 & 0,007 & 2367,795 & 2367,865 & 2379,352 \\
EGu & 0,069 & 0,110 & 1,040 & 0,504 & 29,810 & 0,008 & 2370,186 & 2370,256 & 2381,743 \\
TEV & 0,057 & 0,144 & 0,916 & 0,462 & 48,566 & 0,008 & 2370,615 & 2370,685 & 2382,172 \\
GGu3 & 0,066 & 0,277 & 1,119 & 0,618 & 130,112 & 0,009 & 2374,145 & 2374,215 & 2385,702 \\
ExpGama3 & 0,069 & 0,107 & 1,031 & 0,496 & 28,170 & 0,008 & 2370,013 & 2370,083 & 2381,570 \\
GLIV & 0,066 & 0,096 & 0,969 & 0,446 & 23,249 & 0,008 & 2371,520 & 2371,636 & 2386,929 \\
TCEV & 0,066 & 0,048 & 0,801 & 0,325 & 3,351 & 0,006 & 2370,168 & 2370,343 & 2389,429 \\
\hline \hline
\end{tabular}

A Figura 4.22 apresenta os gráficos qqplot para cada uma das distribuições estudadas. Os gráficos qqplot com envelope simulado das distribuições Gumbel, EGu, TEV, GGu3, ExpGama3 e GLIV apresentam pontos fora do envelope o que sugere não tenham sido amostradas de tais distribuições. Por outro lado, os gráficos qqplot das distribuições GEV e TCEV acomodam todas as observações dentro do envelope. Assim os gráficos qqplot corroboram as indicações das medidas de qualidade de ajuste de que as distribuições GEV e TCEV se ajustam melhor ao conjunto de dados.

A Figura 4.23 mostra os gráficos do logaritmo da verossimilhança perfilada para o(os) parâmetro(os) adicionais das diversas distribuições generalizadas. Para as distribuições EGu e ExpGama3 as curvas são pouco côncavas resultando em erros padrão grandes.Para a distribuição TEV a curva apresenta um comportamento similiar ao da aplicação anterior com um ponto de inflexão, um ponto de mínimo local e dois pontos de máximo local e, da mesma forma, pode ocorrer a convergência para um máximo local dependendo do valor inicial para a otimização. Como na aplicação anterior, o gráfico do logaritmo da verossimilhança perfilada para $\alpha$ da distribuição GGu3 apresenta crescimento lento para valores grandes de $\alpha$. Observa-se que a estimativa de $\alpha$ na distribuição GGu3 é 11892,399 e a função é crescente para este valor de $\alpha$, devido à dependência da precisão usada no algoritmo de estimação. Como anteriormente, porque a estimativa do parâmetro é um valor grande e o logaritmo da verossimilhança perfilada para este parâmetro é uma função de crescimento lento, a estimação do erro padrão fica comprometida. O mesmo comportamento de crescimento lento ocorre no gráfico da distribuição GLIV na direção do parâmetro $\beta$. Porém, diferentemente da aplicação anterior, como a estimativa de $\beta$ não é um valor grande não há dificuldades numéricas com a estimação do erro padrão e o erro padrão é grande devido a falta de concavidade da curva. Para a distribuição TCEV a curva apresenta um ponto de inflexão, provavelmente por questões numéricas, que não parece dificultar a otimização. A curva apresenta boa concavidade. Para a distribuição do valor extremo generalizada (GEV) o gráfico apresenta curva regular como na aplicação anterior (sem ponto de inflexão, multimodalidade ou crescimento lento) e mais côncava. 

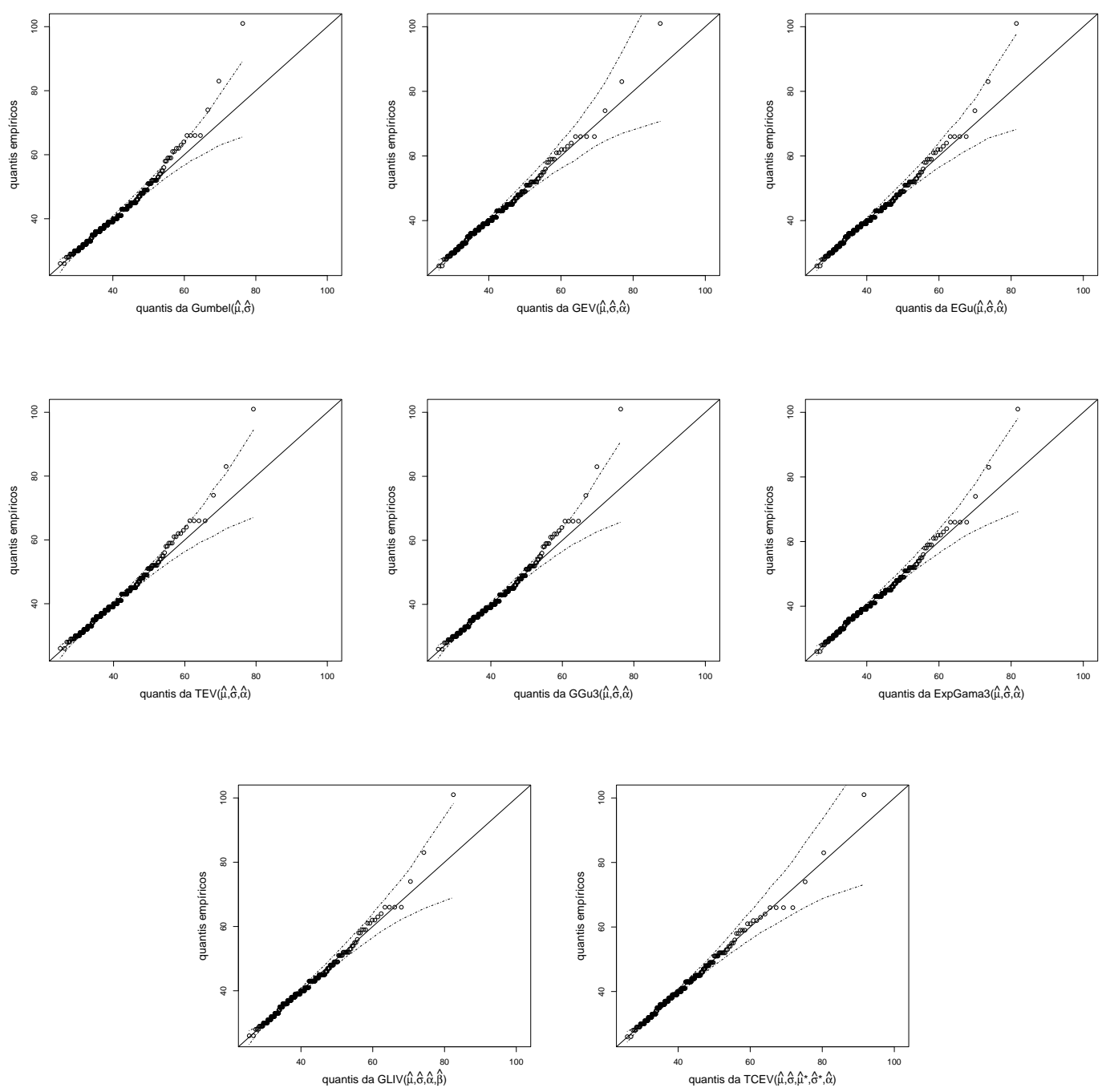

Figura 4.22: QQplots - velocidade máxima mensal do vento (milhas/hora) em West Palm Beach na Flórida de 1984 a 2012

\subsection{Conclusões}

Neste capítulo fazemos uma revisão de distribuições existentes na literatura que têm como caso particular ou limite a distribuição Gumbel. Relacionamos suas características como momentos, método de geração de observações, coeficiente de assimetria e de curtose, identificabilidade e índice da cauda. Tais generalizações possibilitam que a assimetria e a curtose variem diferentemente da distribuição Gumbel cujos valores são constantes. As generalizações são distribuições de variação rápida e índice da cauda igual a zero exceto a distribuição valor extremo generalizada quando o parâmetro adicional é positivo, que é de variação regular e seu índice da cauda pode ser qualquer número real positivo. Apresentamos algumas medidas usuais de qualidade de ajuste. Ajustamos as famílias distribuições identificáveis a um conjunto de dados simulados de uma distribuição Gumbel e a um conjunto de dados reais de velocidade máxima mensal de vento. Comparamos os ajustes através das medidas de qualidade de ajuste apresentadas e avaliamos o comportamento do 

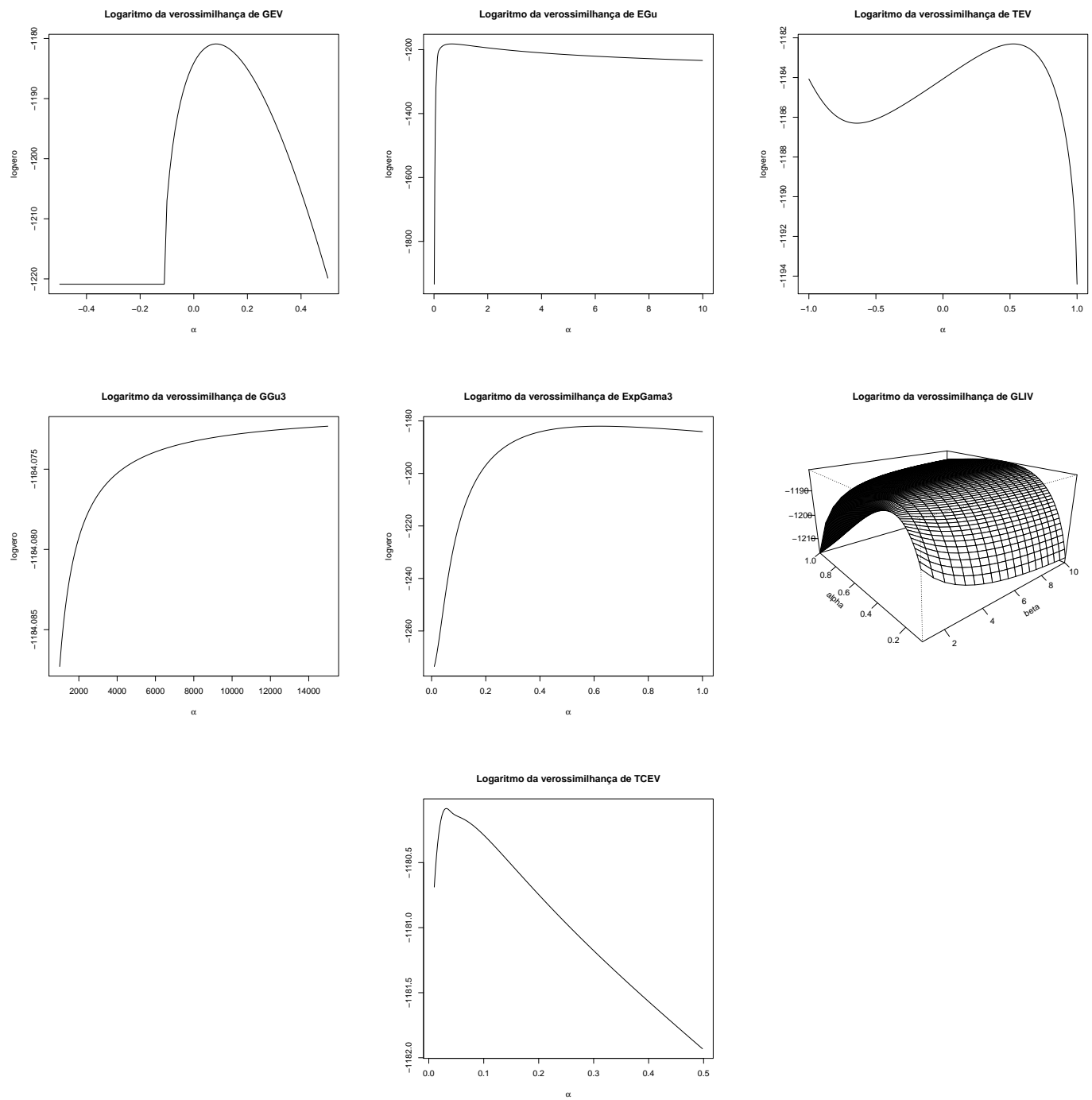

Figura 4.23: Logaritmo da verossimilhança perfilada - velocidade máxima mensal do vento (milhas/hora) em West Palm Beach na Flórida de 1984 a 2012

logaritmo da verossimilhança perfilada do(os) parâmetro(os) adicional(ais) das distribuições. Outros conjuntos de dados de velocidade de vento, não apresentados aqui, foram ajustados às famílias de distribuições identificáveis e exibiram comportamento similar. Concluímos que quando os dados provêem de uma distribuição Gumbel, todas as generalizações são similares em relação a qualidade de ajuste. Quando os dados apresentam valores de coeficiente de assimetria e de curtose bem maiores do que os respectivos valores da distribuição Gumbel, as distribuições valor extremo generalizada e mistura de dois componentes da distribuição valor extremo são as que apresentam melhor qualidade de ajuste. Quando considera-se o princípio da parcimônia a distribuição valor extremo generalizada é a mais indicada para tal situação. 


\section{Capítulo 5}

\section{Conclusão}

Esta tese contempla alguns aspectos da área da Estatística que trata de valores extremos.

Com o objetivo de aprimorar a acurácia da inferência para dados de valores extremos, obtivemos as quantidades necessárias para o cálculo da estatística da razão de verossimilhanças ajustada proposta por Skovgaard (2001) para o modelo e regressão valor extremo proposto por Barreto-Souza \& Vasconcellos (2011) que permite precisão variável e especificações não lineares para a estrutura de regressão da média e da precisão. A obtenção de tais quantidades é bem menos trabalhosa que a das correções de Bartlett. É de se destacar que nossos resultados são bem gerais. Por exemplo, no caso de modelos de regressão valor extremo com precisão variável, os resultados não se limitam a testes de heteroscedasticidade (precisão variável), mas abrangem testes de quaisquer hipóteses nulas que estabelecem um vetor de valores fixados para uma parte do vetor de parâmetros do modelo. Apresentamos resultados de simulações de Monte Carlo implementadas na linguagem Ox para os testes da razão de verossimilhanças, o teste de Wald, o teste escore, o teste gradiente e o teste da razão de verossimilhanças ajustado. Os resultados das simulações mostraram que o teste da razão de verossimilhanças e o teste de Wald podem ser consideravelmente liberais se a amostra não for grande o suficiente para garantir a proximidade entre a distribuição da estatística do teste e a distribuição $\chi^{2}$ limite. O teste gradiente é menos liberal do que os anteriores e o teste de escore pode ser conservativo. As simulações indicaram que o teste ajustado proposto é mais confiável, em termos de controle da probabilidade de erro de tipo I, do que o teste da razão de verossimilhanças original. A estatística ajustada proposta, sob a hipótese nula, têm distribuição muito próxima da distribuição $\chi^{2}$ de referência. Consequentemente, as taxas de rejeição da hipótese nula do teste que usa a estatística ajustada é próxima do respectivo nível nominal. Aplicações do modelo de regressão valor extremo a conjunto de dados reais são apresentadas. A aplicação ilustra o fato de que o modelo de regressão valor extremo pode ser uma opção apropriada para ajustar dados de valores máximos de observações. Mostramos, ainda, que alteração inferencial pode ocorrer dependendo da estatística em que esta se baseia. É recomendável que, quando o teste da razão de verossimilhanças produz valor- $p$ assintótico não muito distante dos níveis nominais usuais, as estatísticas ajustadas sejam utilizadas, especialmente se o tamanho da amostra não for muito grande. As dificuldades computacionais para a produção das estatísticas ajustadas para um conjunto de dados reais limitam-se a implementar a leitura destes no programa utilizado para as simulações.

Para teste unilateral de um dos parâmetros, a estatística usualmente utilizada é a estatística da razão de verossimilhanças sinalizada. Obtivemos os ajuste de Barndorff-Nielsen $(1986,1991)$ para esta esta- 
tística para o caso particular homoscedástico do modelo citado acima, caso em que é possível identificar uma estatística ancilar. Obtivemos, ainda, neste caso particular do modelo, as aproximações para o ajuste de Barndorff-Nielsen (1986, 1991) propostas por DiCiccio \& Martin (1993), Skovgaard (1996), Severini (1999) e Fraser et al. (1999). Nos estudos de simulação, o teste ajustado proposto por Barndorff-Nielsen (1986, 1991) e as aproximações propostas por DiCiccio \& Martin (1993) e Fraser et al. (1999) apresentam as menores discrepâncias em relação à assíntota, com os erros do tipo I próximos dos níveis nominais. Para o caso geral do modelo citado acima obtivemos as aproximações mencionadas. No caso linear heterocedástico os teste propostos por Skovgaard (1996) e Fraser et al. (1999) apresentaram os melhores desempenhos nos resultados de simulação, enquanto no caso não linear homoscedástico contemplado foram os testes Severini (1999) e Fraser et al. (1999). Ressaltamos que em todas as configurações tratadas aqui, o teste proposto por Fraser et al. (1999) esteve entre os melhores. A aplicação a conjuntos de dados reais resultou em diferentes inferências. Recomenda-se, como no caso anterior, que os testes ajustados sejam utilizados quando o tamanho da amostra for moderado ou pequeno e o teste da razão de verossimilhanças sinalizada apresentar valor- $p$ próximo do nível nominal de interesse.

Sem pretender esgotar o assunto, fizemos uma coletânea das distribuições existentes na literatura que têm a distribuição valor extremo máximo ou Gumbel como caso particular ou limite. Relacionamos seus momentos, coeficiente de assimetria e de curtose, índice de variação e índice da cauda. Verificamos quais modelos não são identificáveis. Todas as generalizações têm assimetria e curtose flexíveis. A distribuição valor extremo generalizada se diferencia das outras sendo a única que não é de variação rápida. Ajustamos os modelos identificáveis a um conjunto de dados simulados e a um conjunto de dados reais. Todas as distribuições se ajustaram bem ao conjunto de dados simulados de uma distribuição Gumbel. O conjunto de dados reais utilizado tem o valor dos coeficientes de assimetria e de curtose bem superiores aos respectivos valores da distribuição Gumbel, com presença não desprezível de valores bem discrepantes na cauda direita. Neste caso as distribuições valor extremo generalizada e a mistura de duas distribuições valor extremo apresentaram os menores valores das medidas de qualidade de ajuste. A distribuição valor extremo generalizada apresentou os menores valores dos critérios de informação. A distribuição valor extremo generalizada e a mistura de duas distribuições Gumbel produziram melhores ajustes aos dados do que as outras distribuições na presença não desprezível de observações discrepantes que não podem ser acomodadas pela distribuição Gumbel e, portanto, sugerimos que tais distribuições devem ser utilizadas neste contexto.

Em trabalho futuro pretendemos:

1. elaborar estudos de simulação para comparar a qualidade de ajuste das distribuições investigadas a conjuntos de dados com presença relevante de observações muito discrepantes;

2. fazer outras aplicações a conjuntos de dados com tais características que não estejam relacionados a valores extremos;

3. propor um modelo de regressão valor extremo generalizado para incorporar as características favoráveis de flexibilidade desta distribuição e estudar aspectos de inferência e diagnóstico no modelo proposto; 
4. e ainda, pretendemos implementar os testes ajustados propostos nesta tese no software $\mathrm{R}$ (R Development Core Team, 2013). 


\section{Apêndice A}

\section{Cálculo da função escore e das matrizes de informação para va- lor extremo máximo}

As derivadas parciais do logaritmo da função densidade (2.5) são:

$$
\begin{gathered}
\frac{\partial \ell_{t}\left(\mu_{t}, \sigma_{t}\right)}{\partial \mu_{t}}=\frac{1}{\sigma_{t}}-\frac{1}{\sigma_{t}} \exp \left(-\frac{y_{t}-\mu_{t}}{\sigma_{t}}\right) \\
\frac{\partial^{2} \ell_{t}\left(\mu_{t}, \sigma_{t}\right)}{\partial \mu_{t}^{2}}=-\frac{1}{\sigma_{t}^{2}} \exp \left(-\frac{y_{t}-\mu_{t}}{\sigma_{t}}\right) \\
\frac{\partial \ell_{t}\left(\mu_{t}, \sigma_{t}\right)}{\partial \sigma_{t}}=\frac{1}{\sigma_{t}}\left(-1+\frac{y_{t}-\mu_{t}}{\sigma_{t}}-\exp \left(-\frac{y_{t}-\mu_{t}}{\sigma_{t}}\right)\left(\frac{y_{t}-\mu_{t}}{\sigma_{t}}\right)\right) \\
\frac{\partial^{2} \ell_{t}\left(\mu_{t}, \sigma_{t}\right)}{\partial \sigma_{t}^{2}}=\frac{1}{\sigma_{t}^{2}}\left(1-2\left(\frac{y_{t}-\mu_{t}}{\sigma_{t}}\right)+2 \exp \left(-\frac{y_{t}-\mu_{t}}{\sigma_{t}}\right)\left(\frac{y_{t}-\mu_{t}}{\sigma_{t}}\right)-\exp \left(-\frac{y_{t}-\mu_{t}}{\sigma_{t}}\right)\left(\frac{y_{t}-\mu_{t}}{\sigma_{t}}\right)^{2}\right)
\end{gathered}
$$

$\mathrm{e}$

$$
\frac{\partial^{2} \ell_{t}\left(\mu_{t}, \sigma_{t}\right)}{\partial \mu_{t} \partial \sigma_{t}}=-\frac{1}{\sigma_{t}^{2}}\left(1-\exp \left(-\frac{y_{t}-\mu_{t}}{\sigma_{t}}\right)+\left(\frac{y_{t}-\mu_{t}}{\sigma_{t}}\right) \exp \left(-\frac{y_{t}-\mu_{t}}{\sigma_{t}}\right)\right)
$$

E ainda, por (2.2) e (2.3),

$$
\begin{aligned}
\frac{\partial^{2} \mu_{t}}{\partial \eta_{t}^{2}} & =\frac{\partial}{\partial \eta_{t}}\left(\frac{\partial \mu_{t}}{\partial \eta_{t}}\right)=\frac{\partial}{\partial \eta_{t}}\left(\frac{1}{\frac{\partial \eta_{t}}{\partial \mu_{t}}}\right)=\frac{\partial}{\partial \eta_{t}}\left(\frac{1}{g^{\prime}\left(\mu_{t}\right)}\right)=\frac{\partial}{\partial \eta_{t}}\left(\frac{1}{g^{\prime}\left(g^{-1}\left(\eta_{t}\right)\right)}\right) \\
& =-\frac{1}{g^{\prime}\left(\mu_{t}\right)^{2}} g^{\prime \prime}\left(\mu_{t}\right) \frac{\partial g^{-1}\left(\eta_{t}\right)}{\partial \eta_{t}}=-\frac{1}{g^{\prime}\left(\mu_{t}\right)^{2}} g^{\prime \prime}\left(\mu_{t}\right) \frac{1}{g^{\prime}\left(\mu_{t}\right)}=-\frac{g^{\prime \prime}\left(\mu_{t}\right)}{g^{\prime}\left(\mu_{t}\right)^{3}}
\end{aligned}
$$


e, analogamente,

$$
\begin{aligned}
\frac{\partial^{2} \sigma_{t}}{\partial \delta_{t}^{2}} & =\frac{\partial}{\partial \delta_{t}}\left(\frac{\partial \sigma_{t}}{\partial \delta_{t}}\right)=\frac{\partial}{\partial \delta_{t}}\left(\frac{1}{\frac{\partial \delta_{t}}{\partial \sigma_{t}}}\right)=\frac{\partial}{\partial \delta_{t}}\left(\frac{1}{h^{\prime}\left(\sigma_{t}\right)}\right)=\frac{\partial}{\partial \delta_{t}}\left(\frac{1}{h^{\prime}\left(h^{-1}\left(\delta_{t}\right)\right)}\right) \\
& =-\frac{1}{h^{\prime}\left(\sigma_{t}\right)^{2}} h^{\prime \prime}\left(\sigma_{t}\right) \frac{\partial h^{-1}\left(\delta_{t}\right)}{\partial \delta_{t}}=-\frac{1}{h^{\prime}\left(\sigma_{t}\right)^{2}} h^{\prime \prime}\left(\sigma_{t}\right) \frac{1}{h^{\prime}\left(\sigma_{t}\right)}=-\frac{h^{\prime \prime}\left(\sigma_{t}\right)}{h^{\prime}\left(\sigma_{t}\right)^{3}}
\end{aligned}
$$

De (2.2) e (2.4) obtemos, para $i=1, \ldots, k$, que

$$
\frac{\partial \ell(\beta, \gamma)}{\partial \beta_{i}}=\sum_{t=1}^{n} \frac{\partial \ell_{t}\left(\mu_{t}, \sigma_{t}\right)}{\partial \beta_{i}}=\sum_{t=1}^{n}\left\{\frac{\partial \ell_{t}\left(\mu_{t}, \sigma_{t}\right)}{\partial \mu_{t}} \frac{\partial \mu_{t}}{\partial \eta_{t}} \frac{\partial \eta_{t}}{\partial \beta_{i}}\right\}
$$

ou seja, substituindo (A.1) na equação acima e obtendo as outras derivadas parciais a partir de (2.2), temos que

$$
\frac{\partial \ell(\beta, \gamma)}{\partial \beta_{i}}=\sum_{t=1}^{n}\left\{\left(\frac{1}{\sigma_{t}}-\frac{1}{\sigma_{t}} \exp \left(-\frac{y_{t}-\mu_{t}}{\sigma_{t}}\right)\right) \frac{1}{g^{\prime}\left(\mu_{t}\right)} \frac{\partial \eta_{t}}{\partial \beta_{i}}\right\},
$$

portanto, a forma matricial do vetor escore relativo ao parâmetro $\beta$ é dada por

$$
U_{\beta}(\beta, \gamma)=X^{\top} \sigma^{-1} T\left(\mathcal{I}-\mathcal{Z}^{\dagger}\right) \iota
$$

De (2.3) e (2.4) obtemos, para $i=1, \ldots, m$, que

$$
\frac{\partial \ell(\beta, \gamma)}{\partial \gamma_{i}}=\sum_{t=1}^{n} \frac{\partial \ell_{t}\left(\mu_{t}, \sigma_{t}\right)}{\partial \gamma_{i}}=\sum_{t=1}^{n}\left\{\frac{\partial \ell_{t}\left(\mu_{t}, \sigma_{t}\right)}{\partial \sigma_{t}} \frac{\partial \sigma_{t}}{\partial \delta_{t}} \frac{\partial \delta_{t}}{\partial \gamma_{i}}\right\}
$$

ou seja, substituindo (A.3) na equação acima e obtendo as outras derivadas parciais a partir de (2.3) temos que

$$
\frac{\partial \ell(\beta, \gamma)}{\partial \gamma_{i}}=\sum_{t=1}^{n}\left\{\frac{1}{\sigma_{t}}\left(-1+\frac{y_{t}-\mu_{t}}{\sigma_{t}}-\exp \left(-\frac{y_{t}-\mu_{t}}{\sigma_{t}}\right)\left(\frac{y_{t}-\mu_{t}}{\sigma_{t}}\right)\right) \frac{1}{h^{\prime}\left(\sigma_{t}\right)} \frac{\partial \delta_{t}}{\partial \gamma_{i}}\right\}
$$

portanto, a forma matricial do vetor escore relativo ao parâmetro $\gamma$ é dada por

$$
U_{\gamma}(\beta, \gamma)=Z^{\top} \sigma^{-1} H\left(-\mathcal{I}+\mathcal{Z}-\mathcal{Z} \mathcal{Z}^{\dagger}\right) \iota
$$

De (2.2) e (2.4) obtemos, para $i, j=1, \ldots, k$, que

$$
\begin{aligned}
& \frac{\partial^{2} \ell(\beta, \sigma)}{\partial \beta_{i} \partial \beta_{j}}=\sum_{t=1}^{n}\left\{\frac{\partial^{2} \ell_{t}\left(\mu_{t}, \sigma_{t}\right)}{\partial \beta_{i} \partial \beta_{j}}\right\}=\sum_{t=1}^{n}\left\{\frac{\partial}{\partial \beta_{j}}\left(\frac{\partial \ell_{t}\left(\mu_{t}, \sigma_{t}\right)}{\partial \mu_{t}} \frac{\partial \mu_{t}}{\partial \eta_{t}} \frac{\partial \eta_{t}}{\partial \beta_{i}}\right)\right\} \\
= & \sum_{t=1}^{n}\left\{\frac{\partial}{\partial \beta_{j}}\left(\frac{\partial \ell_{t}\left(\mu_{t}, \sigma_{t}\right)}{\partial \mu_{t}} \frac{\partial \mu_{t}}{\partial \eta_{t}}\right) \frac{\partial \eta_{t}}{\partial \beta_{i}}+\frac{\partial \ell_{t}\left(\mu_{t}, \sigma_{t}\right)}{\partial \mu_{t}} \frac{\partial \mu_{t}}{\partial \eta_{t}} \frac{\partial}{\partial \beta_{j}}\left(\frac{\partial \eta_{t}}{\partial \beta_{i}}\right)\right\} \\
= & \sum_{t=1}^{n}\left\{\left[\frac{\partial}{\partial \beta_{j}}\left(\frac{\partial \ell_{t}\left(\mu_{t}, \sigma_{t}\right)}{\partial \mu_{t}}\right) \frac{\partial \mu_{t}}{\partial \eta_{t}}+\frac{\partial \ell_{t}\left(\mu_{t}, \sigma_{t}\right)}{\partial \mu_{t}} \frac{\partial}{\partial \beta_{j}}\left(\frac{\partial \mu_{t}}{\partial \eta_{t}}\right)\right] \frac{\partial \eta_{t}}{\partial \beta_{i}}+\frac{\partial \ell_{t}\left(\mu_{t}, \sigma_{t}\right)}{\partial \mu_{t}} \frac{\partial \mu_{t}}{\partial \eta_{t}} \frac{\partial^{2} \eta_{t}}{\partial \beta_{i} \partial \beta_{j}}\right\}
\end{aligned}
$$




$$
\begin{aligned}
& =\sum_{t=1}^{n}\left\{\left[\frac{\partial^{2} \ell_{t}\left(\mu_{t}, \sigma_{t}\right)}{\partial \mu_{t}^{2}} \frac{\partial \mu_{t}}{\partial \eta_{t}} \frac{\partial \eta_{t}}{\partial \beta_{j}} \frac{\partial \mu_{t}}{\partial \eta_{t}}+\frac{\partial \ell_{t}\left(\mu_{t}, \sigma_{t}\right)}{\partial \mu_{t}} \frac{\partial^{2} \mu_{t}}{\partial \eta_{t}^{2}} \frac{\partial \eta_{t}}{\partial \beta_{j}}\right] \frac{\partial \eta_{t}}{\partial \beta_{i}}+\frac{\partial \ell_{t}\left(\mu_{t}, \sigma_{t}\right)}{\partial \mu_{t}} \frac{\partial \mu_{t}}{\partial \eta_{t}} \frac{\partial^{2} \eta_{t}}{\partial \beta_{i} \partial \beta_{j}}\right\} \\
& =\sum_{t=1}^{n}\left\{\left[\frac{\partial^{2} \ell_{t}\left(\mu_{t}, \sigma_{t}\right)}{\partial \mu_{t}^{2}}\left(\frac{\partial \mu_{t}}{\partial \eta_{t}}\right)^{2}+\frac{\partial \ell_{t}\left(\mu_{t}, \sigma_{t}\right)}{\partial \mu_{t}} \frac{\partial^{2} \mu_{t}}{\partial \eta_{t}^{2}}\right] \frac{\partial \eta_{t}}{\partial \beta_{i}} \frac{\partial \eta_{t}}{\partial \beta_{j}}+\frac{\partial \ell_{t}\left(\mu_{t}, \sigma_{t}\right)}{\partial \mu_{t}} \frac{\partial \mu_{t}}{\partial \eta_{t}} \frac{\partial^{2} \eta_{t}}{\partial \beta_{i} \partial \beta_{j}}\right\} .
\end{aligned}
$$

Substituindo (A.1), (A.2), e (A.6) na equação acima e obtendo as outras derivadas parciais a partir de (2.2) temos que

$$
\begin{aligned}
\frac{\partial^{2} \ell(\beta, \sigma)}{\partial \beta_{i} \partial \beta_{j}}= & \sum_{t=1}^{n}\left\{\left[\left(-\frac{1}{\sigma_{t}^{2}} \exp \left(-\frac{y_{t}-\mu_{t}}{\sigma_{t}}\right)\right)\left(\frac{1}{g^{\prime}\left(\mu_{t}\right)}\right)^{2}+\left(\frac{1}{\sigma_{t}}-\frac{1}{\sigma_{t}} \exp \left(-\frac{y_{t}-\mu_{t}}{\sigma_{t}}\right)\right)\left(-\frac{g^{\prime \prime}\left(\mu_{t}\right)}{g^{\prime}\left(\mu_{t}\right)^{3}}\right)\right]\right. \\
& \left.\frac{\partial \eta_{t}}{\partial \beta_{i}} \frac{\partial \eta_{t}}{\partial \beta_{j}}+\left(\frac{1}{\sigma_{t}}-\frac{1}{\sigma_{t}} \exp \left(-\frac{y_{t}-\mu_{t}}{\sigma_{t}}\right)\right) \frac{1}{g^{\prime}\left(\mu_{t}\right)} \frac{\partial^{2} \eta_{t}}{\partial \beta_{i} \partial \beta_{j}}\right\} .
\end{aligned}
$$

Logo, na forma matricial,

$$
J_{\beta \beta}=-\frac{\partial^{2} \ell(\beta, \gamma)}{\partial \beta \partial \beta^{\top}}=X^{\top} \sigma^{-1} T\left(\mathcal{Z}^{\dagger} \sigma^{-1}+\left(\mathcal{I}-\mathcal{Z}^{\dagger}\right) S T\right) T X-\left[\iota^{\top}\left(\mathcal{I}-\mathcal{Z}^{\dagger}\right) T \sigma^{-1}\right][\dot{X}]
$$

a definição do produto colchete $[\cdot][\cdot]$ é dada na Seção 2.1 .

A partir de (2.2), (2.3) e (2.4) obtemos também que

$$
\begin{aligned}
\frac{\partial^{2} \ell(\beta, \gamma)}{\partial \beta_{i} \partial \gamma_{j}} & =\sum_{t=1}^{n} \frac{\partial^{2} \ell_{t}\left(\mu_{t}, \sigma_{t}\right)}{\partial \beta_{i} \partial \gamma_{j}}=\sum_{t=1}^{n}\left\{\frac{\partial}{\partial \gamma_{j}}\left[\frac{\partial \ell_{t}\left(\mu_{t}, \sigma_{t}\right)}{\partial \mu_{t}} \frac{\partial \mu_{t}}{\partial \eta_{t}} \frac{\partial \eta_{t}}{\partial \beta_{i}}\right]\right\} \\
& =\sum_{t=1}^{n}\left\{\frac{\partial}{\partial \gamma_{j}}\left[\frac{\partial \ell_{t}\left(\mu_{t}, \sigma_{t}\right)}{\partial \mu_{t}}\right] \frac{\partial \mu_{t}}{\partial \eta_{t}} \frac{\partial \eta_{t}}{\partial \beta_{i}}\right\}=\sum_{t=1}^{n}\left\{\frac{\partial}{\partial \sigma_{t}}\left(\frac{\partial \ell_{t}\left(\mu_{t}, \sigma_{t}\right)}{\partial \mu_{t}}\right) \frac{\partial \sigma_{t}}{\partial \delta_{t}} \frac{\partial \delta_{t}}{\partial \gamma_{j}} \frac{\partial \mu_{t}}{\partial \eta_{t}} \frac{\partial \eta_{t}}{\partial \beta_{i}}\right\} \\
& =\sum_{t=1}^{n}\left\{\frac{\partial^{2} \ell_{t}\left(\mu_{t}, \sigma_{t}\right)}{\partial \mu_{t} \partial \sigma_{t}} \frac{\partial \sigma_{t}}{\partial \delta_{t}} \frac{\partial \delta_{t}}{\partial \gamma_{j}} \frac{\partial \mu_{t}}{\partial \eta_{t}} \frac{\partial \eta_{t}}{\partial \beta_{i}}\right\} .
\end{aligned}
$$

Substituindo (A.5) na equação acima e obtendo as outras derivadas parciais a partir de (2.2) e (2.3) concluímos que

$$
\frac{\partial^{2} \ell(\beta, \gamma)}{\partial \beta_{i} \partial \gamma_{j}}=\sum_{t=1}^{n}\left\{\left[-\frac{1}{\sigma_{t}^{2}}\left(1-\exp \left(-\frac{y_{t}-\mu_{t}}{\sigma_{t}}\right)+\left(\frac{y_{t}-\mu_{t}}{\sigma_{t}}\right) \exp \left(-\frac{y_{t}-\mu_{t}}{\sigma_{t}}\right)\right)\right] \frac{1}{h^{\prime}\left(\sigma_{t}\right)} \frac{\partial \delta_{t}}{\partial \gamma_{j}} \frac{1}{g^{\prime}\left(\mu_{t}\right)} \frac{\partial \eta_{t}}{\partial \beta_{i}}\right\} \text {. }
$$

Logo, na forma matricial,

$$
J_{\beta \gamma}=-\frac{\partial^{2} \ell(\beta, \gamma)}{\partial \beta \partial \gamma^{\top}}=J_{\gamma \beta}^{\top}=X^{\top} \sigma^{-1} T\left(\mathcal{I}-\mathcal{Z}^{\dagger}+\mathcal{Z} \mathcal{Z}^{\dagger}\right) H \sigma^{-1} Z
$$

De (2.3) e (2.4) obtemos, analogamente,

$$
\frac{\partial^{2} \ell(\mu, \sigma)}{\partial \gamma_{i} \partial \gamma_{j}}=\sum_{t=1}^{n}\left\{\left[\frac{\partial^{2} \ell_{t}\left(\mu_{t}, \sigma_{t}\right)}{\partial \sigma_{t}^{2}}\left(\frac{\partial \sigma_{t}}{\partial \delta_{t}}\right)^{2}+\frac{\partial \ell_{t}\left(\mu_{t}, \sigma_{t}\right)}{\partial \sigma_{t}} \frac{\partial^{2} \sigma_{t}}{\partial \delta_{t}^{2}}\right] \frac{\partial \delta_{t}}{\partial \gamma_{i}} \frac{\partial \delta_{t}}{\partial \gamma_{j}}+\frac{\partial \ell_{t}\left(\mu_{t}, \sigma_{t}\right)}{\partial \sigma_{t}} \frac{\partial \sigma_{t}}{\partial \delta_{t}} \frac{\partial^{2} \delta_{t}}{\partial \gamma_{i} \partial \gamma_{j}}\right\}
$$


Substituindo (A.3), (A.4), e (A.7) na equação acima e obtendo as outras derivadas parciais a partir de (2.3) obtemos

$$
\begin{gathered}
\frac{\partial^{2} \ell(\beta, \gamma)}{\partial \gamma_{i} \partial \gamma_{j}}= \\
\sum_{t=1}^{n}\left\{\left[\left(\frac{1}{\sigma_{t}^{2}}\left(1-2\left(\frac{y_{t}-\mu_{t}}{\sigma_{t}}\right)+2 \exp \left(-\frac{y_{t}-\mu_{t}}{\sigma_{t}}\right)\left(\frac{y_{t}-\mu_{t}}{\sigma_{t}}\right)-\exp \left(-\frac{y_{t}-\mu_{t}}{\sigma_{t}}\right)\left(\frac{y_{t}-\mu_{t}}{\sigma_{t}}\right)^{2}\right)\right)\right.\right. \\
\left.\left(\frac{1}{h^{\prime}\left(\sigma_{t}\right)}\right)^{2}+\left(\frac{1}{\sigma_{t}}\left(-1+\frac{y_{t}-\mu_{t}}{\sigma_{t}}-\exp \left(-\frac{y_{t}-\mu_{t}}{\sigma_{t}}\right)\left(\frac{y_{t}-\mu_{t}}{\sigma_{t}}\right)\right)\right)\left(-\frac{h^{\prime \prime}\left(\sigma_{t}\right)}{h^{\prime}\left(\sigma_{t}\right)^{3}}\right)\right] \frac{\partial \delta_{t}}{\partial \gamma_{i}} \frac{\partial \delta_{t}}{\partial \gamma_{j}} \\
\left.+\left(\frac{1}{\sigma_{t}}\left(-1+\frac{y_{t}-\mu_{t}}{\sigma_{t}}-\exp \left(-\frac{y_{t}-\mu_{t}}{\sigma_{t}}\right)\left(\frac{y_{t}-\mu_{t}}{\sigma_{t}}\right)\right)\right) \frac{1}{h^{\prime}\left(\sigma_{t}\right)} \frac{\partial^{2} \delta_{t}}{\partial \gamma_{i} \partial \gamma_{j}}\right\} .
\end{gathered}
$$

Logo, na forma matricial,

$$
\begin{aligned}
J_{\gamma \gamma}= & -\frac{\partial^{2} \ell(\beta, \gamma)}{\partial \gamma \partial \gamma^{\top}}=Z^{\top} \sigma^{-1} H\left(\left(-\mathcal{I}+2 \mathcal{Z}-2 \mathcal{Z} \mathcal{Z}^{\dagger}+\mathcal{Z}^{2} \mathcal{Z}^{\dagger}\right) \sigma^{-1}+\left(-\mathcal{I}+\mathcal{Z}-\mathcal{Z} \mathcal{Z}^{\dagger}\right) Q H\right) H Z+ \\
& {\left[\iota^{\top}\left(\mathcal{I}-\mathcal{Z}+\mathcal{Z} \mathcal{Z}^{\dagger}\right) H \sigma^{-1}\right][\dot{Z}] . }
\end{aligned}
$$

Por (C.3), (C.4), (C.6) e (C.7) temos que

$$
\begin{aligned}
I_{\beta \beta}= & \mathrm{E}_{\omega}\left(J_{\beta \beta}\right)=\mathrm{E}_{\omega}\left(X^{\top} \sigma^{-1} T\left(\mathcal{Z}^{\dagger} \sigma^{-1}+2\left(\mathcal{I}-\mathcal{Z}^{\dagger}\right) S T\right) T X-\left[\iota^{\top}\left(\mathcal{I}-\mathcal{Z}^{\dagger}\right) T \sigma^{-1}\right][\dot{X}]\right) \\
= & X^{\top} \sigma^{-1} T\left(\mathcal{I} \sigma^{-1}+2(\mathcal{I}-\mathcal{I}) S T\right) T X-\left[\iota^{\top}(\mathcal{I}-\mathcal{I}) T \sigma^{-1}\right][\dot{X}]=X^{\top} \sigma^{-1} T^{2} \sigma^{-1} X, \\
& I_{\beta \gamma}=\mathrm{E}_{\omega}\left(J_{\beta \gamma}\right)=\mathrm{E}_{\omega}\left(X^{\top} \sigma^{-1} T\left(\mathcal{I}-\mathcal{Z}^{\dagger}+\mathcal{Z} \mathcal{Z}^{\dagger}\right) H \sigma^{-1} Z\right) \\
= & X^{\top} \sigma^{-1} T(\mathcal{I}-\mathcal{I}+(\mathcal{E}-1) \mathcal{I}) H \sigma^{-1} Z=(\mathcal{E}-1) X^{\top} \sigma^{-1} T H \sigma^{-1} Z, \\
I_{\gamma \gamma}= & \mathrm{E}_{\omega}\left(J_{\gamma \gamma}\right) \\
= & \mathrm{E}_{\omega}\left(Z^{\top} \sigma^{-1} H\left(\left(-\mathcal{I}+2 \mathcal{Z}-2 \mathcal{Z} \mathcal{Z}^{\dagger}+\mathcal{Z}^{2} \mathcal{Z}^{\dagger}\right) \sigma^{-1}+2\left(-\mathcal{I}+\mathcal{Z}-\mathcal{Z} \mathcal{Z}^{\dagger}\right) Q H^{2}\right) H Z+\right. \\
& {\left.\left[\iota^{\top}\left(\mathcal{I}-\mathcal{Z}+\mathcal{Z} \mathcal{Z}^{\dagger}\right) H \sigma^{-1}\right][\dot{Z}]\right) } \\
= & Z^{\top} \sigma^{-1} H\left(\left(-\mathcal{I}+2 \mathcal{E} \mathcal{I}-2(\mathcal{E}-1) \mathcal{I}+\Gamma^{(2)}(2) \mathcal{I}\right) \sigma^{-1}+2(-\mathcal{I}+\mathcal{E} \mathcal{I}-(\mathcal{E}-1) \mathcal{I}) Q\right) H Z \\
& +\left[\iota^{\top}(\mathcal{I}-\mathcal{E} \mathcal{I}+(\mathcal{E}-1) \mathcal{I}) H \sigma^{-1}\right][\dot{Z}]=\left(1+\Gamma^{(2)}(2)\right) Z^{\top} \sigma^{-1} H^{2} \sigma^{-1} Z .
\end{aligned}
$$




\section{Apêndice B}

\section{Cálculo de $q$ e $\Upsilon$ - valor extremo máximo}

Se $v=(y-\mu) / \sigma$ e $v_{1}=\left(y-\mu_{1}\right) / \sigma_{1}$ então podemos escrever $v=\left(\sigma_{1} / \sigma\right) v_{1}+\left(\mu_{1}-\mu\right) / \sigma$. Logo,

$$
\begin{gathered}
\mathfrak{z}=C \mathfrak{z}_{1}+D \iota, \\
\mathcal{Z}=C \mathcal{Z}_{1}+D, \\
\mathfrak{z}^{\dagger}=D^{\dagger} e_{1}
\end{gathered}
$$

$\mathrm{e}$

$$
\mathcal{Z}_{\mathfrak{z}}^{\dagger}=C D^{\dagger} \mathcal{Z}_{1} e_{1}+D D^{\dagger} e_{1}
$$

em que $\mathfrak{z}=\left(z_{1}, \ldots, z_{n}\right)^{\top}, \quad \mathfrak{z}_{1}=\left(z_{11}, \ldots, z_{1 n}\right)^{\top}, \quad z_{1 t}=\left(y_{t}-\mu_{1 t}\right) / \sigma_{1 t}, \quad \mathfrak{z}^{\dagger}=$ $\left(\exp z_{1}, \ldots, \exp z_{n}\right)^{\top}, e_{1}=\left(\exp \left(-\left(\sigma_{11} / \sigma_{1}\right) z_{11}\right), \ldots, \exp \left(-\left(\sigma_{1 n} / \sigma_{n}\right) z_{1 n}\right)\right)^{\top}, \mathcal{Z}_{1}=\operatorname{diag}\left\{z_{11}, \ldots, z_{1 n}\right\}$, $C=\operatorname{diag}\left\{\sigma_{11} / \sigma_{1}, \ldots, \sigma_{1 n} / \sigma_{n}\right\}, D=\operatorname{diag}\left\{\left(\mu_{11}-\mu_{1}\right) / \sigma_{1}, \ldots,\left(\mu_{1 n}-\mu_{n}\right) / \sigma_{n}\right\}, D^{\dagger}=\operatorname{diag}\left\{\exp \left(-\left(\mu_{11}-\right.\right.\right.$ $\left.\left.\left.\mu_{1}\right) / \sigma_{1}\right), \ldots, \exp \left(-\left(\mu_{1 n}-\mu_{n}\right) / \sigma_{n}\right)\right\}$ e $z_{t}, \iota$ e $\mathcal{Z}$ são como definidos no Capítulo 2.1 .

A quantidade (2.21) pode ser escrita como

$$
q=\mathrm{E}_{\omega_{1}}\left[U\left(\omega_{1}\right)\left(\ell\left(\omega_{1}\right)-\ell(\omega)\right)\right]=\left[\begin{array}{c}
\mathrm{E}_{\omega_{1}}\left[U_{\beta}\left(\omega_{1}\right) \ell\left(\omega_{1}\right)\right]-\mathrm{E}_{\omega_{1}}\left[U_{\beta}\left(\omega_{1}\right) \ell(\omega)\right] \\
\mathrm{E}_{\omega_{1}}\left[U_{\gamma}\left(\omega_{1}\right) \ell\left(\omega_{1}\right)\right]-\mathrm{E}_{\omega_{1}}\left[U_{\gamma}\left(\omega_{1}\right) \ell\left(\omega_{1}\right)\right]
\end{array}\right]
$$

Por (2.6), (2.8), (C.1), (C.2), (C.9) e (C.11) temos que

$$
\begin{aligned}
\mathrm{E}_{\omega_{1}}\left[U_{\beta}\left(\omega_{1}\right) \ell\left(\omega_{1}\right)\right] & =\mathrm{E}_{\omega_{1}}\left\{X_{1}^{\top} \sigma_{1}^{-1} T_{1}\left(\iota-\mathfrak{z}_{1}^{\dagger}\right)\left[-l_{1}^{\top}-\mathfrak{z}_{1}^{\top}-\mathfrak{z}_{1}^{\dagger \top}\right] \iota\right\} \\
& =X_{1}^{\top} \sigma_{1}^{-1} T_{1}\left\{-\iota l_{1}^{\top}-\iota \mathrm{E}_{\omega_{1}}\left(\mathfrak{z}_{1}^{\top}\right)-\iota \mathrm{E}_{\omega_{1}}\left(\mathfrak{z}_{1}^{\dagger \top}\right)+\mathrm{E}_{\omega_{1}}\left(\mathfrak{z}_{1}^{\dagger}\right) l_{1}^{\top}+\mathrm{E}_{\omega_{1}}\left(\mathfrak{z}_{1}^{\dagger} \mathfrak{z}_{1}^{\top}\right)+\mathrm{E}_{\omega_{1}}\left(\mathfrak{z}_{1}^{\dagger} \mathfrak{z}_{1}^{\dagger \top}\right)\right\} \iota \\
& =X_{1}^{\top} \sigma_{1}^{-1} T_{1}\left\{-\iota l_{1}^{\top}-\mathcal{E}_{\iota} \iota \iota^{\top}-\iota \iota^{\top}+\iota l_{1}^{\top}+\left(\mathcal{E}_{\iota \iota}^{\top}-\mathcal{I}\right)+(\iota \iota\right. \\
& =0 \iota
\end{aligned}
$$

em que $\mathfrak{z}_{1}^{\dagger}=\mathcal{Z}_{1}^{\dagger} \iota=\left(\exp z_{11}, \ldots, \exp z_{1 n}\right)^{\top} l_{1}=\Phi_{1}^{*} \iota=\left(\ln \sigma_{11}, \ldots, \ln \sigma_{1 n}\right)^{\top}$ sendo $\Phi_{1}^{*}=$ $\operatorname{diag}\left\{\ln \sigma_{11}, \ldots, \ln \sigma_{1 n}\right\}$ e $\mathcal{Z}_{1}^{\dagger}=\operatorname{diag}\left\{\exp \left(-z_{11}\right), \ldots, \exp \left(-z_{1 n}\right)\right\}$ respectivamente análogos a $\Phi^{*}$ e $\mathcal{Z}^{\dagger}$ 
definidos na Seção 2.1. Usando ainda (B.1), (B.3), (C.8) e (C.10), temos que

$$
\begin{aligned}
\mathrm{E}_{\omega_{1}}\left[U_{\beta}\left(\omega_{1}\right) \ell(\omega)\right]= & \mathrm{E}_{\omega_{1}}\left\{X_{1}^{\top} \sigma_{1}^{-1} T_{1}\left(\iota-\mathfrak{z}_{1}^{\dagger}\right)\left[-l^{\top}-\mathfrak{z}^{\top}-\mathfrak{z}^{\dagger \top}\right] \iota\right\} \\
= & X_{1}^{\top} \sigma_{1}^{-1} T_{1}\left\{-\iota l^{\top}-\iota \mathrm{E}_{\omega_{1}}\left(\mathfrak{z}_{1}^{\top}\right) C-\iota \iota^{\top} D-\iota \mathrm{E}_{\omega_{1}}\left(e_{1}^{\top}\right) D^{\dagger}+\mathrm{E}_{\omega_{1}}\left(\mathfrak{z}_{1}^{\dagger}\right) l^{\top}+\mathrm{E}_{\omega_{1}}\left(\mathfrak{z}_{1}^{\dagger} \mathfrak{z}_{1}^{\top}\right) C+\right. \\
& \left.\mathrm{E}_{\omega_{1}}\left(\mathfrak{z}_{1}^{\dagger}\right) \iota^{\top} D+\mathrm{E}_{\omega_{1}}\left(\mathfrak{z}_{1}^{\dagger} e_{1}^{\top}\right) D^{\dagger}\right\} \iota \\
= & X_{1}^{\top} \sigma_{1}^{-1} T_{1}\left\{-\iota l^{\top}-\mathcal{E}_{\iota \iota}^{\top} C-\iota \iota^{\top} D-\iota\left(\iota^{\top} M\right) D^{\dagger}+\iota l^{\top}+\left(\mathcal{E}_{\iota} \iota^{\top}-\mathcal{I}\right) C+\iota \iota^{\top} D+\right. \\
& \left.\left(\iota \iota^{\top} M+C M\right) D^{\dagger}\right\} \iota \\
= & X_{1}^{\top} \sigma_{1}^{-1} T_{1} C\left(M D^{\dagger}-\mathcal{I}\right) \iota
\end{aligned}
$$

em que $\mathfrak{z}^{\dagger}=\mathcal{Z}^{\dagger} \iota=\left(\exp z_{1}, \ldots, \exp z_{1}\right)^{\top}, l=\Phi^{*} \iota=\left(\ln \sigma_{1}, \ldots, \ln \sigma_{n}\right)^{\top}$ sendo $\mathcal{Z}^{\dagger}$ e $\Phi^{*}$ como definidos na Seção 2.1 e $M=\operatorname{diag}\left\{\Gamma\left(1+\sigma_{11} / \sigma_{1}\right), \ldots, \Gamma\left(1+\sigma_{1 n} / \sigma_{n}\right)\right\}$. Então,

$$
\mathrm{E}_{\omega_{1}}\left[U_{\beta}\left(\omega_{1}\right) l\left(\omega_{1}\right)\right]-\mathrm{E}_{\omega_{1}}\left[U_{\beta}\left(\omega_{1}\right) l(\omega)\right]=X_{1}^{\top} \sigma_{1}^{-1} T_{1} C\left(\mathcal{I}-M D^{\dagger}\right) \iota
$$

Por (2.6), (2.9), (C.1), (C.2), (C.5), (C.12), (C.14), (C.18) e (C.20), temos que

$$
\begin{aligned}
\mathrm{E}_{\omega_{1}}\left[U_{\gamma}\left(\omega_{1}\right) \ell\left(\omega_{1}\right)\right]= & \mathrm{E}_{\omega_{1}}\left\{Z_{1}^{\top} \sigma_{1}^{-1} H_{1}\left(\mathfrak{z}_{1}-\mathcal{Z}_{1} \mathfrak{z}_{1}^{\dagger}-\iota\right)\left[-l_{1}^{\top}-\mathfrak{z}_{1}^{\top}-\mathfrak{z}_{1}^{\dagger \top}\right] \iota\right\} \\
= & Z_{1}^{\top} \sigma_{1}^{-1} H_{1}\left\{-\mathrm{E}_{\omega_{1}}\left(\mathfrak{z}_{1}\right) l_{1}^{\top}-\mathrm{E}_{\omega_{1}}\left(\mathfrak{z}_{1} \mathfrak{z}_{1}^{\top}\right)-\mathrm{E}_{\omega_{1}}\left(\mathfrak{z}_{1} \mathfrak{z}_{1}^{\dagger \top}\right)+\mathrm{E}_{\omega_{1}}\left(\mathcal{Z}_{1} \mathfrak{z}_{1}^{\dagger}\right) l_{1}^{\top}\right. \\
& \left.+\mathrm{E}_{\omega_{1}}\left(\mathcal{Z}_{1} \mathfrak{z}_{1}^{\dagger} \mathfrak{z}_{1}^{\top}\right)+\mathrm{E}_{\omega_{1}}\left(\mathcal{Z}_{1} \mathfrak{z}_{1}^{\dagger} \mathfrak{z}_{1}^{\dagger \top}\right)+\iota l_{1}^{\top}+\iota \mathrm{E}_{\omega_{1}}\left(\mathfrak{z}_{1}^{\top}\right)+\iota \mathrm{E}_{\omega_{1}}\left(\mathfrak{z}_{1}^{\dagger \top}\right)\right\} \iota \\
= & Z_{1}^{\top} \sigma_{1}^{-1} H_{1}\left\{-\mathcal{E} \iota l_{1}^{\top}-\left(\mathcal{E}^{2} \iota \iota^{\top}-\mathcal{E}^{2} \mathcal{I}+\Gamma^{(2)}(1) \mathcal{I}\right)-\left(\mathcal{E}_{\iota \iota}^{\top}-\mathcal{I}\right)+(\mathcal{E}-1) \iota l_{1}^{\top}\right. \\
& +\mathcal{E}(\mathcal{E}-1) \iota \iota-\mathcal{E}(\mathcal{E}-1) \mathcal{I}+\Gamma^{(2)}(2) \mathcal{I}+(\mathcal{E}-1) \iota \iota^{\top} \\
& \left.+(\mathcal{E}-2) \mathcal{I}+\iota l_{1}^{\top}+\mathcal{E} \iota \iota^{\top}+\iota \iota^{\top}\right\} \iota \\
= & -Z_{1}^{\top} \sigma_{1}^{-1} H_{1} \iota,
\end{aligned}
$$

lembrando que, $\Gamma^{(2)}(n)=2 \Gamma^{(1)}(n-1)+(n-1) \Gamma^{(2)}(n-1)$ e $\mathcal{E}=-\Gamma^{(1)}(1)$.

Por (2.6), (2.9), (C.2), (C.5), (C.8), (C.13), (C.14),(C.19), (C.20), temos que

$$
\begin{aligned}
\mathrm{E}_{\omega_{1}}\left[U_{\gamma}\left(\omega_{1}\right) \ell(\omega)\right]= & \mathrm{E}_{\omega_{1}}\left\{Z_{1}^{\top} \sigma_{1}^{-1} H_{1}\left(\mathfrak{z}_{1}-\mathcal{Z}_{1} \mathfrak{z}_{1}^{\dagger}-\iota\right)\left[-l^{\top}-\mathfrak{z}^{\top}-\mathfrak{z}^{\dagger \top}\right] \iota\right\} \\
= & \mathrm{E}_{\omega_{1}}\left\{Z_{1}^{\top} \sigma_{1}^{-1} H_{1}\left(\mathfrak{z}_{1}-\mathcal{Z}_{1} \mathfrak{z}_{1}^{\dagger}-\iota\right)\left[-l^{\top}-\mathfrak{z}_{1}^{\top} C-\iota^{\top} D-e_{1}^{\top} D^{\dagger}\right] \iota\right\} \\
= & Z_{1}^{\top} \sigma_{1}^{-1} H_{1}\left\{-\mathrm{E}_{\omega_{1}}\left(\mathfrak{z}_{1}\right) l^{\top}-\mathrm{E}_{\omega_{1}}\left(\mathfrak{z}_{1} \mathfrak{z}_{1}^{\top}\right) C-\mathrm{E}_{\omega_{1}}\left(\mathfrak{z}_{1}\right) \iota^{\top} D-\mathrm{E}_{\omega_{1}}\left(\mathfrak{z}_{1} e_{1}^{\top}\right) D^{\dagger}\right. \\
& +\mathrm{E}_{\omega_{1}}\left(\mathcal{Z}_{1} \mathfrak{z}_{1}^{\dagger}\right) l^{\top}+\mathrm{E}_{\omega_{1}}\left(\mathcal{Z}_{1} \mathfrak{z}_{1}^{\dagger} \mathfrak{z}_{1}^{\top}\right) C+\mathrm{E}_{\omega_{1}}\left(\mathcal{Z}_{1} \mathfrak{z}_{1}^{\dagger}\right) \iota^{\top} D+\mathrm{E}_{\omega_{1}}\left(\mathcal{Z}_{1} \mathfrak{z}_{1}^{\dagger} e_{1}^{\top}\right) D^{\dagger} \\
& \left.+\iota l^{\top}+\iota \mathrm{E}_{\omega_{1}}\left(\mathfrak{z}_{1}^{\top}\right) C+\iota \iota^{\top} D+\iota \mathrm{E}_{\omega_{1}}\left(e_{1}^{\top}\right) D^{\dagger}\right\} \iota \\
= & Z_{1}^{\top} \sigma_{1}^{-1} H_{1}\left\{-\mathcal{E} \iota l^{\top}-\left(\mathcal{E}^{2} \iota \iota^{\top}-\mathcal{E}^{2} \mathcal{I}+\Gamma^{(2)}(1) \mathcal{I}\right) C-\mathcal{E} \iota \iota^{\top} D\right. \\
& -\left(\mathcal{E} \iota \iota^{\top} M-\mathcal{E} M-N\right) D^{\dagger}+(\mathcal{E}-1) \iota l^{\top} \\
& +\left(\mathcal{E}(\mathcal{E}-1) \iota \iota \top-\mathcal{E}(\mathcal{E}-1) \mathcal{I}+\Gamma^{(2)}(2) \mathcal{I}\right) C+(\mathcal{E}-1) \iota \iota^{\top} D \\
& +\left((\mathcal{E}-1) \iota \iota \iota^{\top} M-\mathcal{E} M-(\mathcal{I}+C) N\right) D^{\dagger} \\
& \left.+\iota l^{\top}+\mathcal{E} \iota \iota \iota^{\top} C+\iota \iota \iota^{\top} D+\iota(M \iota)^{\top} D^{\dagger}\right\} \iota
\end{aligned}
$$




$$
=-Z_{1}^{\top} \sigma_{1}^{-1} H_{1} C\left\{\mathcal{E} \mathcal{I}+N D^{\dagger}\right\} \iota
$$

em que $N=\operatorname{diag}\left\{\Gamma^{(1)}\left(1+\sigma_{11} / \sigma_{1}\right), \ldots, \Gamma^{(1)}\left(1+\sigma_{1 n} / \sigma_{n}\right)\right\}$. Então,

$$
\begin{aligned}
\mathrm{E}_{\omega_{1}}\left[U_{\gamma}\left(\omega_{1}\right) \ell\left(\omega_{1}\right)\right]-\mathrm{E}_{\omega_{1}}\left[U_{\gamma}\left(\omega_{1}\right) \ell(\omega)\right] & =-Z_{1}^{\top} \sigma_{1}^{-1} H_{1} \iota-\left(-Z_{1}^{\top} \sigma_{1}^{-1} H_{1} C\left(\mathcal{E} \mathcal{I}+N D^{\dagger}\right) \iota\right) \\
& =-Z_{1}^{\top} \sigma_{1}^{-1} H_{1}\left(\mathcal{I}-C\left(\mathcal{E} \mathcal{I}+N D^{\dagger}\right)\right) \iota \\
& =Z_{1}^{\top} \sigma_{1}^{-1} H_{1}\left(C\left(\mathcal{E} \mathcal{I}+N D^{\dagger}\right)-\mathcal{I}\right) \iota .
\end{aligned}
$$

Segue que

$$
q=\left[\begin{array}{c}
X_{1}^{\top} \sigma_{1}^{-1} T_{1} C\left(\mathcal{I}-M D^{\dagger}\right) \iota \\
Z_{1}^{\top} \sigma_{1}^{-1} H_{1}\left(C\left(\mathcal{E} \mathcal{I}+N D^{\dagger}\right)-\mathcal{I}\right) \iota
\end{array}\right] .
$$

A quantidade (2.22) pode ser escrita como

$$
\Upsilon=\left[\begin{array}{cc}
\mathrm{E}_{\omega_{1}}\left[U_{\beta}\left(\omega_{1}\right) U_{\beta}^{\top}(\omega)\right] & \mathrm{E}_{\omega_{1}}\left[U_{\beta}\left(\omega_{1}\right) U_{\gamma}(\omega)\right] \\
\mathrm{E}_{\omega_{1}}\left[U_{\gamma}\left(\omega_{1}\right) U_{\beta}^{\top}(\omega)\right] & \mathrm{E}_{\omega_{1}}\left[U_{\gamma}\left(\omega_{1}\right) U_{\gamma}^{\top}(\omega)\right]
\end{array}\right]
$$

Por (2.8), (B.3), (C.1), (C.8) e (C.10) temos que

$$
\begin{aligned}
\mathrm{E}_{\omega_{1}}\left\{U_{\beta}\left(\omega_{1}\right) U_{\beta}^{\top}(\omega)\right\} & =\mathrm{E}_{\omega_{1}}\left\{X_{1}^{\top} \sigma_{1}^{-1} T_{1}\left(\iota-\mathfrak{z}_{1}^{\dagger}\right)\left[X^{\top} \sigma^{-1} T\left(\iota-\mathfrak{z}^{\dagger}\right)\right]^{\top}\right\} \\
& =\mathrm{E}_{\omega_{1}}\left\{X_{1}^{\top} \sigma_{1}^{-1} T_{1}\left(\iota-\mathfrak{z}_{1}^{\dagger}\right)\left[X^{\top} \sigma^{-1} T\left(\iota-D^{\dagger} e_{1}\right)\right]^{\top}\right\} \\
& =X_{1}^{\top} \sigma_{1}^{-1} T_{1} \mathrm{E}_{\omega_{1}}\left\{\left(\iota-\mathfrak{z}_{1}^{\dagger}\right)\left[\iota^{\top}-e_{1}^{\top} D^{\dagger}\right]\right\} T \sigma^{-1} X \\
& =X_{1}^{\top} \sigma_{1}^{-1} T_{1}\left(\iota \iota-\iota \iota \iota^{\top} M D^{\dagger}-\iota \iota^{\top}+\left(\iota \iota \iota^{\top} M+C M\right) D^{\dagger}\right) T \sigma^{-1} X \\
& =X_{1}^{\top} \sigma_{1}^{-1} T_{1} C M D^{\dagger} T \sigma^{-1} X
\end{aligned}
$$

Por (2.9), (B.1), (B.4), (C.2), (C.5), (C.8), (C.13), (C.14), (C.15), (C.17), (C.19), (C.20), (C.21) temos que

$$
\begin{aligned}
\mathrm{E}_{\omega_{1}}\left\{U_{\gamma}\left(\omega_{1}\right) U_{\gamma}^{\top}(\omega)\right\}= & \mathrm{E}_{\omega_{1}}\left\{Z_{1}^{\top} \sigma_{1}^{-1} H_{1}\left(\mathfrak{z}_{1}-\mathcal{Z}_{1} \mathfrak{z}_{1}^{\dagger}-\iota\right)\left[Z^{\top} \sigma^{-1} H\left(\mathfrak{z}-\mathcal{Z}_{\mathfrak{z}}^{\dagger}-\iota\right)\right]^{\top}\right\} \\
= & \mathrm{E}_{\omega_{1}}\left\{Z_{1}^{\top} \sigma_{1}^{-1} H_{1}\left(\mathfrak{z}_{1}-\mathcal{Z}_{1} \mathfrak{z}_{1}^{\dagger}-\iota\right)\right. \\
& {\left.\left[Z^{\top} \sigma^{-1} H\left(C \mathfrak{z}_{1}+D \iota-C D^{\dagger} \mathcal{Z}_{1} e_{1}-D D^{\dagger} e_{1}-\iota\right)\right]^{\top}\right\} } \\
= & Z_{1}^{\top} \sigma_{1}^{-1} H_{1}\left\{\left(\mathrm{E}_{\omega_{1}}\left(\mathfrak{z}_{1} \mathfrak{z}_{1}^{\top}\right) C+\mathrm{E}_{\omega_{1}}\left(\mathfrak{z}_{1}\right) \iota^{\top} D-\mathrm{E}_{\omega_{1}}\left(\mathfrak{z}_{1} e_{1}^{\top} \mathcal{Z}_{1}\right) D^{\dagger} C\right.\right. \\
& -\mathrm{E}_{\omega_{1}}\left(\mathfrak{z}_{1} e_{1}^{\top}\right) D^{\dagger} D-\mathrm{E}_{\omega_{1}}\left(\mathfrak{z}_{1}\right) \iota^{\top}-\mathrm{E}_{\omega_{1}}\left(\mathcal{Z}_{1} \mathfrak{z}_{1}^{\dagger} \mathfrak{z}_{1}^{\top}\right) C \\
& -\mathrm{E}_{\omega_{1}}\left(\mathcal{Z}_{1} \mathfrak{z}_{1}^{\dagger}\right) \iota^{\top} D+\mathrm{E}_{\omega_{1}}\left(\mathcal{Z}_{1} \mathfrak{z}_{1}^{\dagger} e_{1}^{\top} \mathcal{Z}_{1}\right) D^{\dagger} C+\mathrm{E}_{\omega_{1}}\left(\mathcal{Z}_{1} \mathfrak{z}_{1}^{\dagger} e_{1}^{\top}\right) D^{\dagger} D \\
& +\mathrm{E}_{\omega_{1}}\left(\mathcal{Z}_{1} \mathfrak{z}_{1}^{\dagger}\right) \iota^{\top}-\iota \mathrm{E}_{\omega_{1}}\left(\mathfrak{z}_{1}^{\top}\right) C-\iota \iota \iota^{\top} D+\iota \mathrm{E}_{\omega_{1}}\left(e_{1}^{\top} \mathcal{Z}_{1}\right) D^{\dagger} C \\
& \left.\left.+\iota \mathrm{E}_{\omega_{1}}\left(e_{1}^{\top}\right) D^{\dagger} D+\iota \iota \iota^{\top}\right)\right\} H \sigma^{-1} Z \\
= & Z_{1}^{\top} \sigma_{1}^{-1} H_{1}\left\{\left(\mathcal{E}^{2} \iota \iota \iota^{\top}-\mathcal{E}^{2} \mathcal{I}+\Gamma^{(2)}(1) \mathcal{I}\right) C+\mathcal{E} \iota \iota \iota^{\top} D-\left(-\mathcal{E} \iota \iota \iota^{\top} N+\mathcal{E} N+P\right) D^{\dagger} C\right. \\
& -\left(\mathcal{E}_{\iota \iota}^{\top} M-\mathcal{E} M-N\right) D^{\dagger} D-(\mathcal{E} \iota) \iota-\left(\mathcal{E}(\mathcal{E}-1) \iota \iota-\mathcal{E}(\mathcal{E}-1) \mathcal{I}+\Gamma^{(2)}(2) \mathcal{I}\right) C
\end{aligned}
$$




$$
\begin{aligned}
& -((\mathcal{E}-1) \iota) \iota^{\top} D \\
& +\left(-(\mathcal{E}-1) \iota \iota^{\top} N+(\mathcal{E}-1) N+2 N+P+C P\right) D^{\dagger} C \\
& +\left((\mathcal{E}-1) \iota \iota^{\top} M-(\mathcal{E}-1) M-M-(\mathcal{I}+C) N\right) D^{\dagger} D \\
& +((\mathcal{E}-1) \iota) \iota^{\top}-\iota\left(\mathcal{E} \iota^{\top}\right) C-\iota \iota^{\top} D+\iota(-N \iota)^{\top} D^{\dagger} C \\
& \left.\left.+\iota(M \iota)^{\top} D^{\dagger} D+\iota \iota^{\top}\right)\right\} H \sigma^{-1} Z \\
= & Z_{1}^{\top} \sigma_{1}^{-1} H_{1} C\left\{\mathcal{E} \mathcal{I}+D^{\dagger}(N+C P-N D)\right\} H \sigma^{-1} Z
\end{aligned}
$$

$P=\operatorname{diag}\left\{\Gamma^{(2)}\left(1+\sigma_{11} / \sigma_{1}\right), \ldots, \Gamma^{(2)}\left(1+\sigma_{1 n} / \sigma_{n}\right)\right\}$.

Por (2.8), (2.9), (B.1), (B.4), (C.1), (C.2), (C.8), (C.10), (C.11), (C.15), (C.16), temos que

$$
\begin{aligned}
\mathrm{E}_{\omega_{1}}\left\{U_{\beta}\left(\omega_{1}\right) U_{\gamma}^{\top}(\omega)\right\}= & \mathrm{E}_{\omega_{1}}\left\{X_{1}^{\top} \sigma_{1}^{-1} T_{1}\left(\iota-\mathfrak{z}_{1}^{\dagger}\right)\left[Z^{\top} \sigma^{-1} H\left(\mathfrak{z}-\mathcal{Z}_{\mathfrak{z}}^{\dagger}-\iota\right)\right]^{\top}\right\} \\
= & \mathrm{E}_{\omega_{1}}\left\{X_{1}^{\top} \sigma_{1}^{-1} T_{1}\left(\iota-\mathfrak{z}_{1}^{\dagger}\right)\left[Z^{\top} \sigma^{-1} H\left(C \mathfrak{z}_{1}+D \iota-C D^{\dagger} \mathcal{Z}_{1} e_{1}-D D^{\dagger} e_{1}-\iota\right)\right]^{\top}\right\} \\
= & \mathrm{E}_{\omega_{1}}\left\{X_{1}^{\top} \sigma_{1}^{-1} T_{1}\left(\iota-\mathfrak{z}_{1}^{\dagger}\right)\left[\left(\mathfrak{z}_{1}^{\top} C+\iota^{\top} D-e_{1}^{\top} \mathcal{Z}_{1} D^{\dagger} C-e_{1}^{\top} D^{\dagger} D-\iota^{\top}\right)\right] H \sigma^{-1} Z\right\} \\
= & X_{1}^{\top} \sigma_{1}^{-1} T_{1}\left\{\iota \mathrm{E}_{\omega_{1}}\left(\mathfrak{z}_{1}^{\top}\right) C+\iota \iota^{\top} D-\iota \mathrm{E}_{\omega_{1}}\left(e_{1}^{\top} \mathcal{Z}_{1}\right) D^{\dagger} C-\iota \mathrm{E}_{\omega_{1}}\left(e_{1}^{\top}\right) D^{\dagger} D\right. \\
& -\iota \iota^{\top}-\mathrm{E}_{\omega_{1}}\left(\mathfrak{z}_{1}^{\dagger} \mathfrak{z}_{1}^{\top}\right) C-\mathrm{E}_{\omega_{1}}\left(\mathfrak{z}_{1}^{\dagger}\right) \iota^{\top} D+\mathrm{E}_{\omega_{1}}\left(\mathfrak{z}_{1}^{\dagger} e_{1}^{\top} \mathcal{Z}_{1}\right) D^{\dagger} C+\mathrm{E}_{\omega_{1}}\left(\mathfrak{z}_{1}^{\dagger} e_{1}^{\top}\right) D^{\dagger} D \\
& \left.+\mathrm{E}_{\omega_{1}}\left(\mathfrak{z}_{1}^{\dagger}\right) \iota^{\top}\right\} H \sigma^{-1} Z \\
= & X_{1}^{\top} \sigma_{1}^{-1} T_{1}\left\{\iota \mathcal{E} \iota \iota^{\top} C+\iota \iota^{\top} D-\iota(-N \iota)^{\top} D^{\dagger} C-\iota(M \iota)^{\top} D^{\dagger} D\right. \\
& -\iota \iota^{\top}-\left(\mathcal{E}_{\iota \iota}^{\top}-\mathcal{I}\right) C-\iota \iota^{\top} D+\left(-\iota \iota^{\top} N-M-C N\right) D^{\dagger} C \\
& \left.+\left(\iota \iota \iota^{\top} M+C M\right) D^{\dagger} D+\iota \iota \iota^{\top}\right\} H \sigma^{-1} Z \\
= & X_{1}^{\top} \sigma_{1}^{-1} T_{1} C\left\{\mathcal{I}+D^{\dagger}(M D-M-C N)\right\} H \sigma^{-1} Z,
\end{aligned}
$$

Usando ainda (C.5), (C.13) e (C.19) temos que

$$
\begin{aligned}
\mathrm{E}_{\omega_{1}}\left\{U_{\gamma}\left(\omega_{1}\right) U_{\beta}^{\top}(\omega)\right\}= & \mathrm{E}_{\omega_{1}}\left\{Z_{1}^{\top} \sigma_{1}^{-1} H_{1}\left(\mathfrak{z}_{1}-\mathcal{Z}_{1} \mathfrak{z}_{1}^{\dagger}-\iota\right)\left[X^{\top} \sigma^{-1} T\left(\iota-\mathfrak{z}^{\dagger}\right)\right]^{\top}\right\} \\
= & \mathrm{E}_{\omega_{1}}\left\{Z_{1}^{\top} \sigma_{1}^{-1} H_{1}\left(\mathfrak{z}_{1}-\mathcal{Z}_{1} \mathfrak{z}_{1}^{\dagger}-\iota\right)\left[X^{\top} \sigma^{-1} T\left(\iota-D^{\dagger} e_{1}\right)\right]^{\top}\right\} \\
= & \mathrm{E}_{\omega_{1}}\left\{Z_{1}^{\top} \sigma_{1}^{-1} H_{1}\left(\mathfrak{z}_{1}-\mathcal{Z}_{1} \mathfrak{z}_{1}^{\dagger}-\iota\right)\left[\left(\iota^{\top}-e_{1}^{\top} D^{\dagger}\right) T \sigma^{-1} X\right]\right\} \\
= & Z_{1}^{\top} \sigma_{1}^{-1} H_{1}\left\{\mathrm{E}_{\omega_{1}}\left(\mathfrak{z}_{1}\right) \iota^{\top}-\mathrm{E}_{\omega_{1}}\left(\mathfrak{z}_{1} e_{1}^{\top}\right) D^{\dagger}-\mathrm{E}_{\omega_{1}}\left(\mathcal{Z}_{1} \mathfrak{z}_{1}^{\dagger}\right) \iota^{\top}+\mathrm{E}_{\omega_{1}}\left(\mathcal{Z}_{1} \mathfrak{z}_{1}^{\dagger} e_{1}^{\top}\right) D^{\dagger}-\iota \iota^{\top}\right. \\
& \left.+\iota \mathrm{E}_{\omega_{1}}\left(e_{1}^{\top}\right) D^{\dagger}\right\} T \sigma^{-1} X \\
= & Z_{1}^{\top} \sigma_{1}^{-1} H_{1}\left\{\mathcal{E}_{\iota} \iota^{\top}-\left(\mathcal{E}_{\iota} \iota^{\top} M-\mathcal{E} M-N\right) D^{\dagger}-((\mathcal{E}-1) \iota) \iota^{\top}+\left((\mathcal{E}-1) \iota \iota^{\top} M\right.\right. \\
& \left.-(\mathcal{E}-1) M-M-(\mathcal{I}+C) N) D^{\dagger}-\iota \iota^{\top}+\iota(M \iota)^{\top} D^{\dagger}\right\} T \sigma^{-1} X \\
= & -Z_{1}^{\top} \sigma_{1}^{-1} H_{1} C N D^{\dagger} T \sigma^{-1} X .
\end{aligned}
$$




\section{Apêndice C}

\section{Cálculo de esperanças das matrizes - valor extremo máximo}

Seja $y \sim E V(\mu, \sigma)$ máximo então $\mathbf{z}=(y-\mu) / \sigma \sim E V(0,1)$ máximo e temos que

$$
\mathrm{E}\left(\mathbf{z}^{n} \exp (-c \mathbf{z})\right)=\int_{-\infty}^{\infty} \mathbf{z}^{n} \exp (-c \mathbf{z}) \exp (-\mathbf{z}-\exp (-\mathbf{z})) d \mathbf{z}
$$

Fazendo $y=\exp (-\mathbf{z})$, temos que

$$
\begin{aligned}
\mathrm{E}\left(\mathbf{z}^{n} \exp (-c \mathbf{z})\right) & =\int_{\infty}^{0}(-\ln y)^{n} y^{c} y \exp (-y) \frac{-1}{y} d y \\
& =\int_{0}^{\infty}(-1)^{n}(\ln y)^{n} y^{c} \exp (-y) d y \\
& =(-1)^{n} \Gamma^{(n)}(1+c)
\end{aligned}
$$

uma vez que $\int_{0}^{\infty} x^{\nu-1} \exp (-\mu x)(\ln x)^{n} d x=\partial^{n}\left(\mu^{-\nu} \Gamma(\nu)\right) / \partial \nu^{n}$ para $n=0,1,2,3, \ldots$ (Gradshteyn \& Ryzhik, 2000, equação 4.358.5). Além disso, $\psi(x)=d \ln \Gamma(x) / d x=\Gamma^{(1)}(x) / \Gamma(x)$ é a função psi então $\Gamma^{(1)}(n)=\Gamma(n) \psi(n)=(n-1) !\left(-\mathcal{E}+\sum_{k=1}^{n-1} 1 / k\right)$ uma vez que $\psi(n+1)=$ $-\mathcal{E}+\sum_{k=1}^{n} 1 / k$ (Gradshteyn \& Ryzhik, 2000, equação 8.365.4). Portanto

$$
\begin{array}{rlrl}
\mathrm{E}(\mathbf{z}) & =(-1)^{1} \Gamma^{(1)}(1+0) & \mathcal{E}, \\
\mathrm{E}\left(\mathbf{z}^{2}\right) & =(-1)^{2} \Gamma^{(2)}(1+0) & & \Gamma^{(2)}(1), \\
\mathrm{E}(\exp (-c \mathbf{z})) & =(-1)^{0} \Gamma^{(0)}(1+c) & & \Gamma(1+c), \\
\mathrm{E}(\exp (-(1+c) \mathbf{z})) & =(-1)^{0} \Gamma^{(0)}(2+c) & & \Gamma(2+c), \\
\mathrm{E}(\mathbf{z} \exp (-\mathbf{z})) & =(-1)^{1} \Gamma^{(1)}(1+1) & & \mathcal{E}-1, \\
\mathrm{E}(\mathbf{z} \exp (-2 \mathbf{z})) & =(-1)^{1} \Gamma^{(1)}(1+2) & & 2 \mathcal{E}-3, \\
\mathrm{E}(\mathbf{z} \exp (-c \mathbf{z})) & =(-1)^{1} \Gamma^{(1)}(1+c) & = & -\Gamma^{(1)}(1+c), \\
\mathrm{E}(\mathbf{z} \exp (-(1+c) \mathbf{z})) & =(-1)^{1} \Gamma^{(1)}(2+c) & = & -\Gamma^{(1)}(2+c), \\
\mathrm{E}\left(\mathbf{z}^{2} \exp (-\mathbf{z})\right) & =(-1)^{2} \Gamma^{(2)}(1+1) & = & \Gamma^{(2)}(2), \\
\mathrm{E}\left(\mathbf{z}^{2} \exp (-c \mathbf{z})\right) & =(-1)^{2} \Gamma^{(2)}(1+c) & = & \Gamma^{(2)}(1+c), \\
\mathrm{E}\left(\mathbf{z}^{2} \exp (-(1+c) \mathbf{z})\right) & =(-1)^{2} \Gamma^{(2)}(2+c) & = & \Gamma^{(2)}(2+c) .
\end{array}
$$


Logo,

$$
\begin{gathered}
\mathrm{E}_{\omega}\left(\mathfrak{z}^{\dagger}\right)=\iota, \\
\mathrm{E}_{\omega}(\mathfrak{z})=\mathcal{E}_{\iota}, \\
\mathrm{E}_{\omega}(\mathcal{Z})=\mathrm{E}_{\omega}\left(\operatorname{diag}\left\{\mathfrak{z}_{1}, \ldots, \mathfrak{z}_{n}\right\}\right) \\
=\mathcal{E} \mathcal{I}, \\
\mathrm{E}_{\omega}\left(\mathcal{Z}^{\dagger}\right)=\mathrm{E}_{\omega}\left(\operatorname{diag}\left\{\exp \left(-\mathfrak{z}_{1}\right), \ldots, \exp \left(-\mathfrak{z}_{n}\right)\right\}\right) \\
=\mathcal{I}, \\
\mathrm{E}_{\omega}\left(\mathcal{Z}_{\mathfrak{z}}^{\dagger}\right)=\mathrm{E}_{\omega}\left(\left(\mathfrak{z}_{1} \exp \left(-\mathfrak{z}_{1}\right), \ldots, \mathfrak{z}_{n} \exp \left(-\mathfrak{z}_{n}\right)\right)^{\top}\right) \\
=(\mathcal{E}-1) \iota, \\
\mathrm{E}_{\omega}\left(\mathcal{Z} \mathcal{Z}^{\dagger}\right)=\mathrm{E}_{\omega} \operatorname{diag}\left\{\mathfrak{z}_{1} \exp \left(-\mathfrak{z}_{1}\right), \ldots, \mathfrak{z}_{n} \exp \left(-\mathfrak{z}_{n}\right)\right\} \\
=(\mathcal{E}-1) \mathcal{I} . \\
\mathrm{E}_{\omega}\left(\mathcal{Z}^{2} \mathcal{Z}^{\dagger}\right)=\mathrm{E}_{\omega} \operatorname{diag}\left\{\mathfrak{z}_{1}^{2} \exp \left(-\mathfrak{z}_{1}\right), \ldots, \mathfrak{z}_{n}^{2} \exp \left(-\mathfrak{z}_{n}\right)\right\} \\
=\Gamma^{(2)}(2) \mathcal{I} . \\
\mathrm{E}_{\omega}\left(e_{1}\right)=\left(\Gamma\left(1+\frac{\sigma_{11}}{\sigma_{1}}\right), \ldots, \Gamma\left(1+\frac{\sigma_{1 n}}{\sigma_{n}}\right)\right)^{\top} \\
= \\
M \iota
\end{gathered}
$$

onde $M=\operatorname{diag}\left\{\Gamma\left(1+\sigma_{11} / \sigma_{1}\right), \ldots, \Gamma\left(1+\sigma_{1 n} / \sigma_{n}\right)\right\}$. Como por hipótese, $y_{i}$ e $y_{j}$ são independentes se $i \neq j$, segue que,

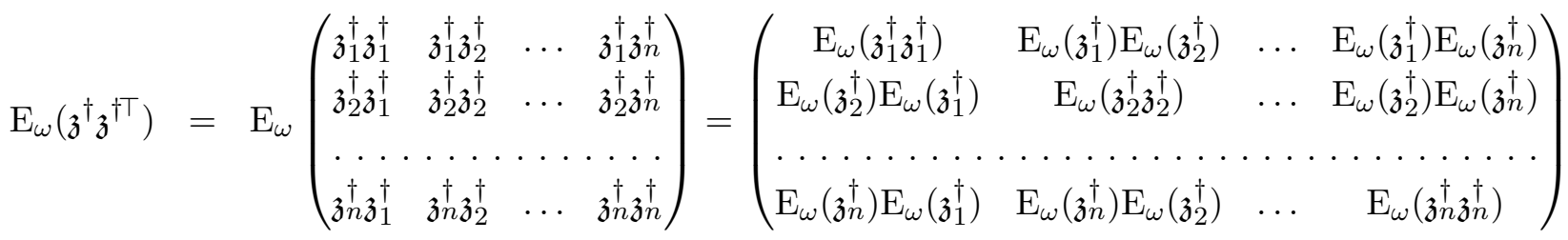

$$
\begin{aligned}
& =\left(\begin{array}{cccc}
2 & 1 & \ldots & 1 \\
1 & 2 & \ldots & 1 \\
\ldots & \ldots & \ldots \\
1 & 1 & \ldots & 2
\end{array}\right)=\iota^{\top}+\mathcal{I}
\end{aligned}
$$




$$
\begin{aligned}
& \mathrm{E}_{\omega}\left(\mathfrak{z}^{\dagger} e_{1}^{\top}\right)=\mathrm{E}_{\omega}\left(\begin{array}{cccc}
\mathfrak{z}_{1}^{\dagger} e_{11} & \mathfrak{z}_{1}^{\dagger} e_{12} & \ldots & \mathfrak{z}_{1}^{\dagger} e_{1 n} \\
\mathfrak{z}_{2}^{\dagger} e_{11} & \mathfrak{z}_{2}^{\dagger} e_{12} & \ldots & \mathfrak{z}_{2}^{\dagger} e_{1 n} \\
\ldots & \ldots \ldots & \ldots & \ldots \\
\ldots & \ldots \\
\mathfrak{z}_{n}^{\dagger} e_{11} & \mathfrak{z}_{n}^{\dagger} e_{12} & \ldots & \mathfrak{z}_{n}^{\dagger} e_{1 n}
\end{array}\right)
\end{aligned}
$$

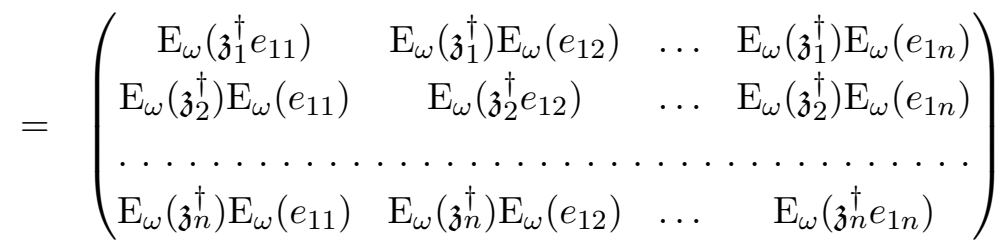

$$
\begin{aligned}
& =\left(\begin{array}{cccc}
\Gamma\left(2+\frac{\sigma_{11}}{\sigma_{1}}\right) & \Gamma\left(1+\frac{\sigma_{12}}{\sigma_{2}}\right) & \ldots & \Gamma\left(1+\frac{\sigma_{1 n}}{\sigma_{n}}\right) \\
\Gamma\left(1+\frac{\sigma_{11}}{\sigma_{1}}\right) & \Gamma\left(2+\frac{\sigma_{12}}{\sigma_{2}}\right) & \ldots & \Gamma\left(1+\frac{\sigma_{1 n}}{\sigma_{n}}\right) \\
\ldots \ldots \ldots & \ldots \ldots \ldots \ldots & \ldots & \ldots \ldots \ldots \\
\Gamma\left(1+\frac{\sigma_{11}}{\sigma_{1}}\right) & \Gamma\left(1+\frac{\sigma_{12}}{\sigma_{2}}\right) & \ldots & \Gamma\left(2+\frac{\sigma_{1 n}}{\sigma_{n}}\right)
\end{array}\right)=\iota \iota^{\top} M+C M, \\
& \mathrm{E}_{\omega}\left(\mathfrak{z}^{\dagger} \mathfrak{z}^{\top}\right)=\mathrm{E}_{\omega}\left(\begin{array}{cccc}
\mathfrak{z}_{1}^{\dagger} \mathfrak{z}_{1} & \mathfrak{z}_{1}^{\dagger} \mathfrak{z}_{2} & \ldots & \mathfrak{z}_{1}^{\dagger} \mathfrak{z}_{n} \\
\mathfrak{z}_{2}^{\dagger} \mathfrak{z}_{1} & \mathfrak{z}_{2}^{\dagger} \mathfrak{z}_{2} & \ldots & \mathfrak{z}_{2}^{\dagger} \mathfrak{z}_{n} \\
\ldots & \ldots & \ldots & \ldots \\
\ldots & \ldots \\
\mathfrak{z}_{n}^{\dagger} \mathfrak{z}_{1} & \mathfrak{z}_{n}^{\dagger} \mathfrak{z}_{2} & \ldots & \mathfrak{z}_{n}^{\dagger} \mathfrak{z}_{n}
\end{array}\right)
\end{aligned}
$$

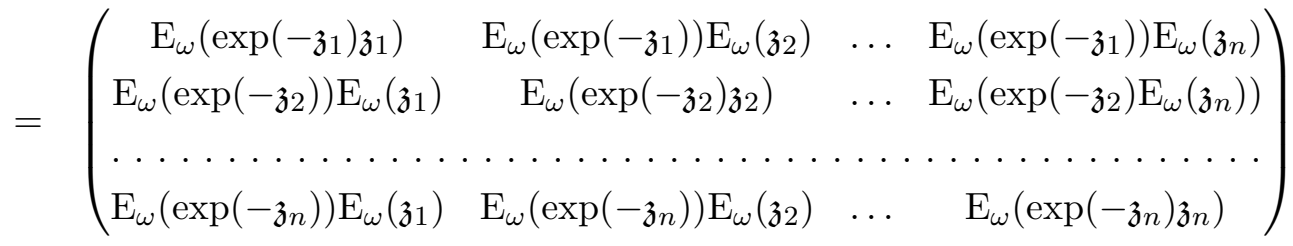

$$
\begin{aligned}
& =\left(\begin{array}{cccc}
\mathcal{E}-1 & \mathcal{E} & \ldots & \mathcal{E} \\
\mathcal{E} & \mathcal{E}-1 & \ldots & \mathcal{E} \\
\ldots & \ldots & \ldots & \ldots \\
\mathcal{E} & \mathcal{E} & \ldots & \mathcal{E}-1
\end{array}\right)=\mathcal{E}_{\iota \iota} \iota^{\top}-\mathcal{I} \\
& \mathrm{E}_{\omega}\left(\mathfrak{z}^{\dagger \top}\right)=\mathrm{E}_{\omega}\left(\mathfrak{z}^{\dagger} \mathfrak{z}^{\top}\right) \\
& \mathrm{E}_{\omega}\left(\mathfrak{z}_{1}^{\top}\right)=\mathrm{E}_{\omega}\left(\begin{array}{cccc}
\mathfrak{z}_{1} e_{11} & \mathfrak{z}_{1} e_{12} & \ldots & \mathfrak{z}_{1} e_{1 n} \\
\mathfrak{z}_{2} e_{11} & \mathfrak{z}_{2} e_{12} & \ldots & \mathfrak{z}_{2} e_{1 n} \\
\ldots \ldots & \ldots \ldots & \ldots & \ldots \\
\mathfrak{z}_{n} e_{11} & \mathfrak{z}_{n} e_{12} & \ldots & \mathfrak{z}_{n} e_{1 n}
\end{array}\right)
\end{aligned}
$$

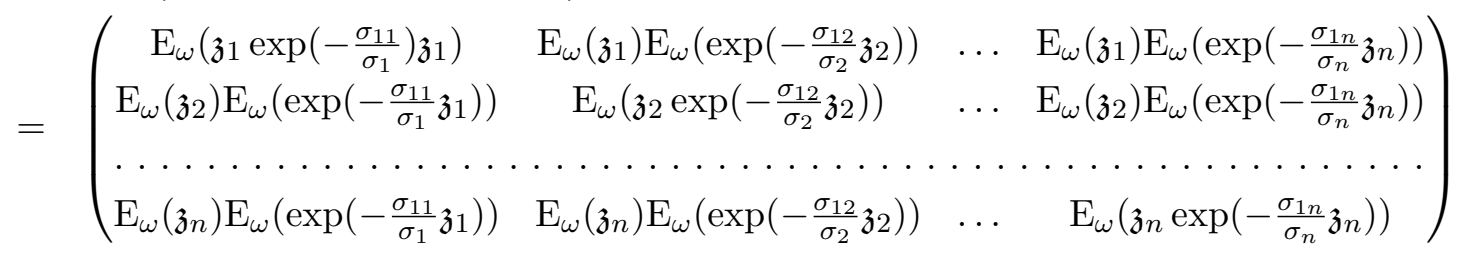




$$
\begin{aligned}
& =\left(\begin{array}{cccc}
-\Gamma^{(1)}\left(1+\frac{\sigma_{11}}{\sigma_{1}}\right) & \mathcal{E} \Gamma\left(1+\frac{\sigma_{12}}{\sigma_{2}}\right) & \ldots & \mathcal{E} \Gamma\left(1+\frac{\sigma_{1 n}}{\sigma_{n}}\right) \\
\mathcal{E} \Gamma\left(1+\frac{\sigma_{11}}{\sigma_{1}}\right) & -\Gamma^{(1)}\left(1+\frac{\sigma_{12}}{\sigma_{2}}\right) & \ldots & \mathcal{E} \Gamma\left(1+\frac{\sigma_{1 n}}{\sigma_{n}}\right) \\
\ldots \ldots \ldots \ldots \ldots \ldots \ldots \ldots \ldots \ldots \ldots \ldots \ldots \ldots & \ldots \ldots \ldots \\
\mathcal{E} \Gamma\left(1+\frac{\sigma_{11}}{\sigma_{1}}\right) & \mathcal{E} \Gamma\left(1+\frac{\sigma_{12}}{\sigma_{2}}\right) & \ldots & -\Gamma^{(1)}\left(1+\frac{\sigma_{1 n}}{\sigma_{n}}\right)
\end{array}\right) \\
& =\mathcal{E} \iota \iota^{\top} M-\mathcal{E} M-N,
\end{aligned}
$$

onde $N=\operatorname{diag}\left\{\Gamma^{(1)}\left(1+\sigma_{11} / \sigma_{1}\right), \ldots, \Gamma^{(1)}\left(1+\sigma_{1 n} / \sigma_{n}\right)\right\}$,

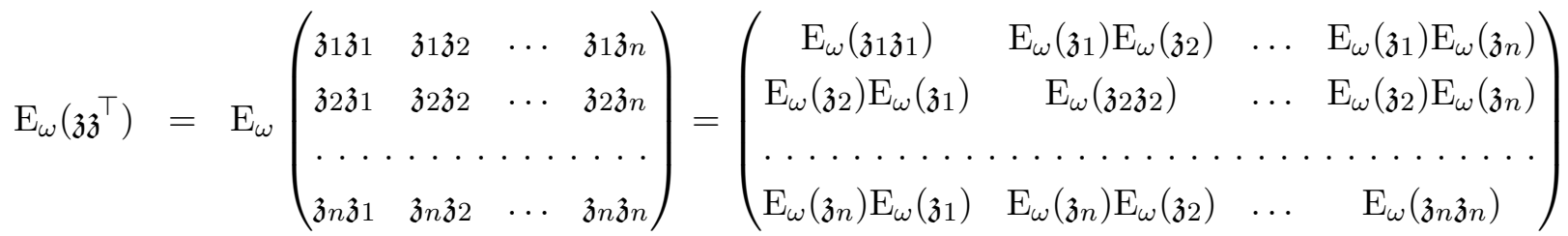

$$
\begin{aligned}
& =\left(\begin{array}{cccc}
\Gamma^{(2)}(1) & \mathcal{E}^{2} & \ldots & \mathcal{E}^{2} \\
\mathcal{E}^{2} & \Gamma^{(2)}(1) & \ldots & \mathcal{E}^{2} \\
\ldots & \ldots & \ldots & \ldots \\
\mathcal{E}^{2} & \mathcal{E}^{2} & \ldots & \Gamma^{(2)}(1)
\end{array}\right)=\mathcal{E}^{2} \iota \iota^{\top}-\mathcal{E}^{2} \mathcal{I}+\Gamma^{(2)}(1) \mathcal{I} \\
& \mathrm{E}_{\omega}\left(\mathcal{Z} e_{1}\right)=\mathrm{E}_{\omega}\left(\mathfrak{z}_{1} \exp \left(-\frac{\sigma_{11}}{\sigma_{1}} \mathfrak{z}_{1}\right), \ldots, \mathfrak{z}_{n} \exp \left(-\frac{\sigma_{1 n}}{\sigma_{n}} \mathfrak{z}_{n}\right)\right)^{\top} \\
& =\left(-\Gamma^{(1)}\left(1+\frac{\sigma_{11}}{\sigma_{1}}\right), \ldots,-\Gamma^{(1)}\left(1+\frac{\sigma_{1 n}}{\sigma_{n}}\right)\right)^{\top}=-N \iota,
\end{aligned}
$$

$$
\begin{aligned}
& \mathrm{E}_{\omega}\left(\mathfrak{z}^{\dagger} e_{1}^{\top} \mathcal{Z}\right)
\end{aligned}
$$

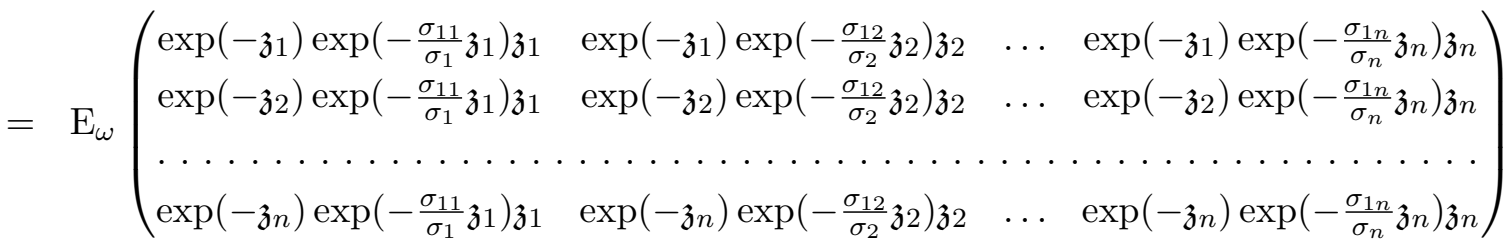

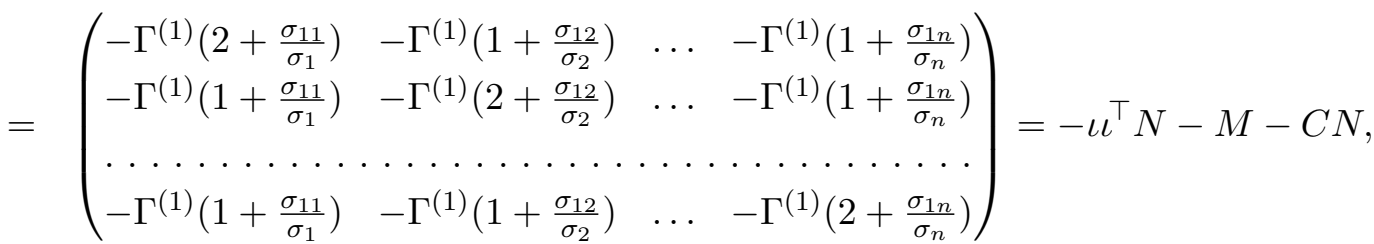

pois, $\Gamma^{(1)}(n)=\Gamma(n-1)+(n-1) \Gamma^{(1)}(n-1)$,

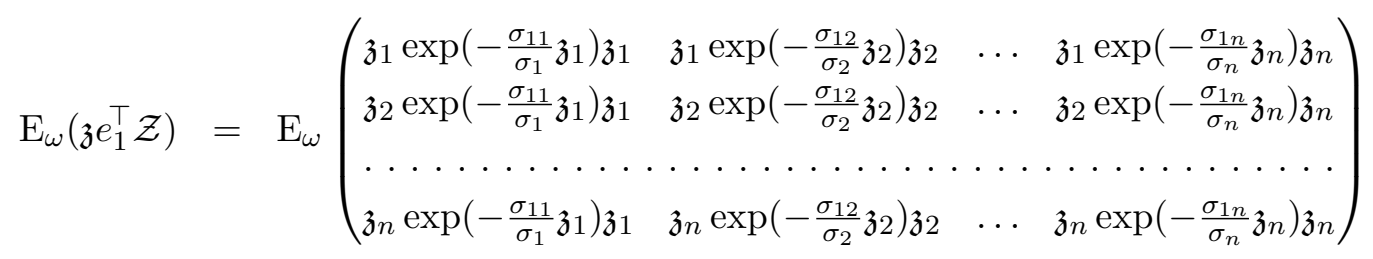




$$
\begin{aligned}
& =\left(\begin{array}{cccc}
\Gamma^{(2)}\left(1+\frac{\sigma_{11}}{\sigma_{1}}\right) & -\mathcal{E} \Gamma^{(1)}\left(1+\frac{\sigma_{12}}{\sigma_{2}}\right) & \ldots & -\mathcal{E} \Gamma^{(1)}\left(1+\frac{\sigma_{1 n}}{\sigma_{n}}\right) \\
-\mathcal{E} \Gamma^{(1)}\left(1+\frac{\sigma_{11}}{\sigma_{1}}\right) & \Gamma^{(2)}\left(1+\frac{\sigma_{12}}{\sigma_{2}}\right) & \ldots & -\mathcal{E} \Gamma^{(1)}\left(1+\frac{\sigma_{1 n}}{\sigma_{n}}\right) \\
\ldots \ldots \ldots \ldots \ldots \ldots \ldots \ldots \ldots \ldots \ldots \ldots \ldots \ldots \ldots & \ldots \ldots \ldots \\
-\mathcal{E} \Gamma^{(1)}\left(1+\frac{\sigma_{11}}{\sigma_{1}}\right) & -\mathcal{E} \Gamma^{(1)}\left(1+\frac{\sigma_{12}}{\sigma_{2}}\right) & \ldots & \Gamma^{(2)}\left(1+\frac{\sigma_{1 n}}{\sigma_{n}}\right)
\end{array}\right) \\
& =-\mathcal{E} \iota \iota^{\top} N+\mathcal{E} N+P,
\end{aligned}
$$

onde $P=\operatorname{diag}\left\{\Gamma^{(2)}\left(1+\sigma_{11} / \sigma_{1}\right), \ldots, \Gamma^{(2)}\left(1+\sigma_{1 n} / \sigma_{n}\right)\right\}$,

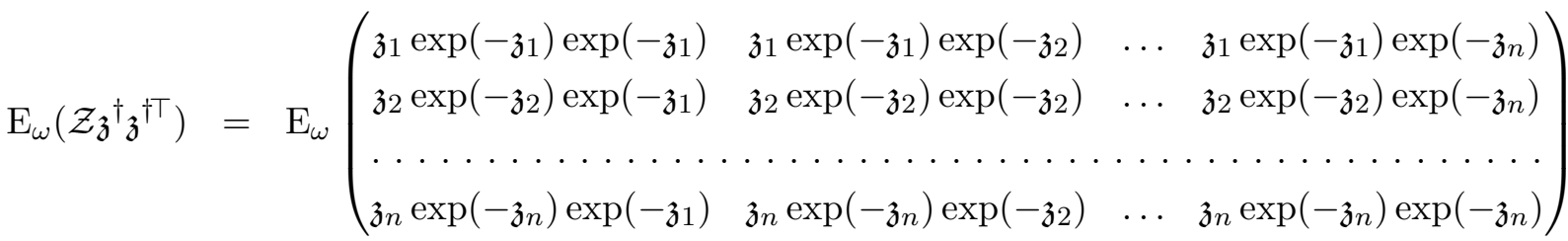

$$
\begin{aligned}
& =\left(\begin{array}{cccc}
2 \mathcal{E}-3 & (\mathcal{E}-1) & \ldots & (\mathcal{E}-1) \\
(\mathcal{E}-1) & 2 \mathcal{E}-3 & \ldots & (\mathcal{E}-1) \\
\ldots \ldots & \ldots & \ldots & \ldots \\
(\mathcal{E}-1) & (\mathcal{E}-1) & \ldots & 2 \mathcal{E}-3
\end{array}\right)=(\mathcal{E}-1) \iota \iota^{\top}+(\mathcal{E}-2) \mathcal{I} . \\
& \mathrm{E}_{\omega}\left(\mathcal{Z}_{\mathfrak{z}}^{\dagger} e_{1}^{\top}\right)
\end{aligned}
$$

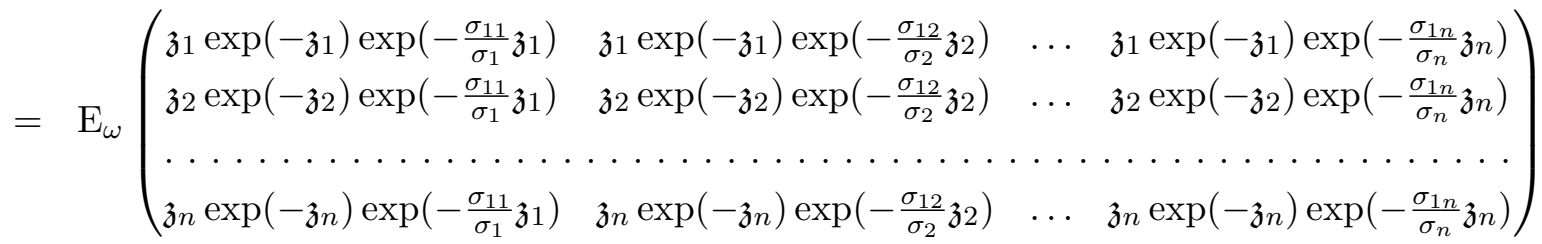

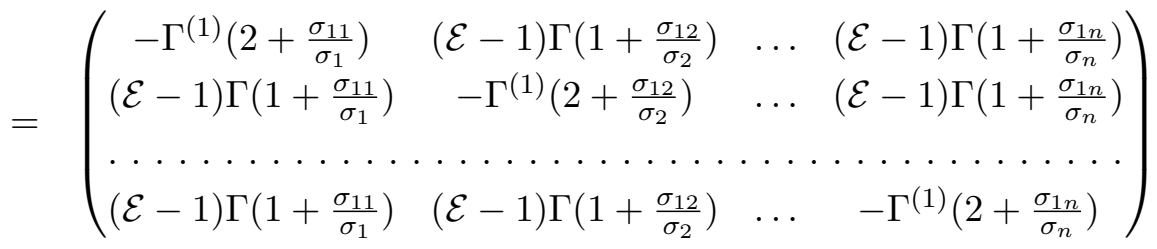

$$
\begin{aligned}
& =(\mathcal{E}-1) \iota^{\top} M-(\mathcal{E}-1) M-M-(\mathcal{I}+C) N
\end{aligned}
$$

pois, $\Gamma^{(1)}(n)=\Gamma(n-1)+(n-1) \Gamma^{(1)}(n-1)$,

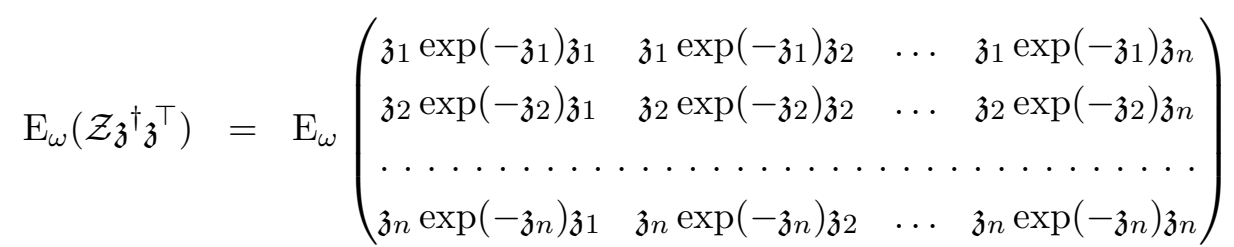

$$
\begin{aligned}
& =\left(\begin{array}{cccc}
\Gamma^{(2)}(2) & \mathcal{E}(\mathcal{E}-1) & \ldots & \mathcal{E}(\mathcal{E}-1) \\
\mathcal{E}(\mathcal{E}-1) & \Gamma^{(2)}(2) & \ldots & \mathcal{E}(\mathcal{E}-1) \\
\ldots \ldots & \ldots \ldots \ldots & \ldots & \ldots \ldots \\
\mathcal{E}(\mathcal{E}-1) & \mathcal{E}(\mathcal{E}-1) & \ldots & \Gamma^{(2)}(2)
\end{array}\right)
\end{aligned}
$$




$$
=\mathcal{E}(\mathcal{E}-1) \iota^{\top}-\mathcal{E}(\mathcal{E}-1) \mathcal{I}+\Gamma^{(2)}(2) \mathcal{I}
$$

$$
\begin{aligned}
& \mathrm{E}_{\omega}\left(\mathcal{Z}_{\mathfrak{z}}^{\dagger} e_{1}^{\top} \mathcal{Z}\right)
\end{aligned}
$$

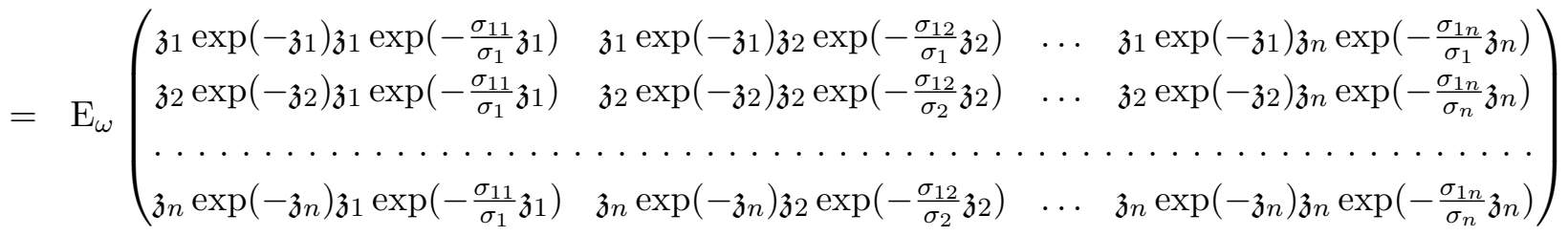

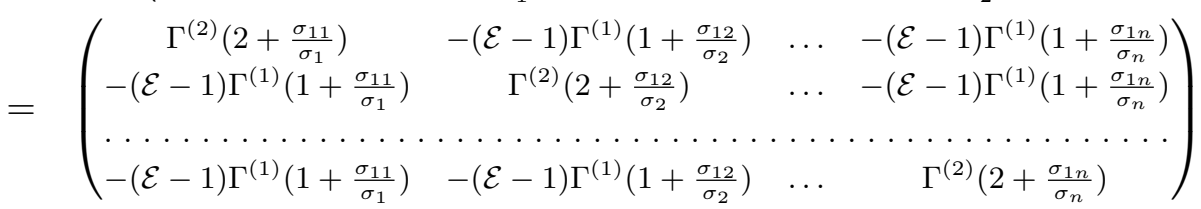

$$
\begin{aligned}
& =-(\mathcal{E}-1) \iota \iota^{\top} N+(\mathcal{E}-1) N+2 N+P+C P \text {, }
\end{aligned}
$$

pois, $\Gamma^{(2)}(n)=2 \Gamma^{(1)}(n-1)+(n-1) \Gamma^{(2)}(n-1)$. 


\section{Apêndice D}

\section{Distribuição das estatísticas}

Considere o caso particular do modelo de regressão valor extremo máximo (2.1)-(2.3) em que a função de ligação $g$ é a função identidade, o componente sistemático do parâmetro de locação é uma função linear e o parâmetro de dispersão é constante (modelo homoscedástico). Seja $y=\left(y_{1}, \ldots, y_{n}\right)^{\top}$ um vetor de variáveis independentes tais que $y_{t} \sim V E\left(\mathbf{x}_{j} \beta, \sigma\right)$ e $\theta=\left(\beta^{\top}, \sigma\right)^{\top}$ o vetor de parâmetros desconhecidos do modelo . No que segue, $\nu=\left(\beta_{1}, \ldots, \beta_{r}\right)^{\top}$ representa o parâmetro de interesse, $\nu^{c}=\left(\beta_{r+1}, \ldots, \beta_{k}\right)^{\top} \mathrm{e}$ $\psi=\left(\nu^{c \top}, \sigma\right)^{\top}$ é o parâmetro de perturbação de dimensão $s$. Note que $\beta=\left(\nu^{\top}, \nu^{c \top}\right)^{\top}$. Aqui, $\left(\widehat{\beta}^{\top}, \widehat{\sigma}^{\top}\right)=$ $\left(\widehat{\nu}^{\top}, \widehat{\psi}^{\top}\right)$ é o estimador de máxima verossimilhança de $\left(\beta^{\top}, \sigma^{\top}\right)=\left(\nu^{\top}, \psi^{\top}\right)$ e $\left(\nu_{0}^{\top}, \widetilde{\psi}^{\top}\right)=\left(\nu_{0}^{\top}, \widetilde{\nu}^{c \top}, \widetilde{\sigma}\right)$ é o estimador de máxima verossimilhança restrito de $\left(\nu^{\top}, \psi^{\top}\right)=\left(\nu_{0}^{\top}, \nu^{c \top}, \sigma\right)$ sob $\mathcal{H}_{0}$.

Resultado 1. Sob as condições definidas acima, $z_{t}=\left(y_{t}-\mu_{t}\right) / \sigma_{t} \sim V E(0,1)$, a distribuição conjunta de $\left(z_{1}, \ldots, z_{n}\right)$ e a distribuição de $W=h\left(z_{1}, \ldots, z_{n}\right), h$ função monótona e diferenciável, não dependem dos parâmetros desconhecidos.

Demonstração. Seja $Y \sim V E(\mu, \sigma)$ e $Z=(Y-\mu) / \sigma$ então $P(Z \leq z)=P((Y-\mu) / \sigma \leq z)=P(Y \leq$ $\sigma z+\mu)=\exp (-\exp (-((\sigma z+\mu)-\mu) / \sigma))=\exp (-\exp (-z))$, isto é, $Z \sim V E(0,1)$. Como $y_{1}, \ldots, y_{n}$ são independentes então $z_{1}\left(y_{1}\right), \ldots, z_{n}\left(y_{n}\right)$ são independentes e a distribuição conjunta de $z_{1}, \ldots, z_{n}$ é o produto das distribuições de $z_{t} \sim V E(0,1) \operatorname{logo}$ não depende de parâmetros. A distribuição de $W$ é função da distribuição conjunta de $z_{1}, \ldots, z_{n}$ (ver Ross, 2002, p. 282) logo não depende de parâmetros.

Resultado 2. Sob as condições definidas acima, $(\widehat{\beta}-\beta) / \sigma$ e $\widehat{\sigma} / \sigma$ são quantidades pivotais. Da Silva et al. (2008, p. 805-806)

Demonstração. No caso em que o parâmetro de dispersão é constante, a função de verossimilhança do modelo de regressão valor extremo máximo com componente sistemático da média linear é

$$
L_{y}(\beta, \sigma)=\prod_{t=1}^{n} f\left(y_{t} ; \beta, \sigma\right)=\prod_{t=1}^{n} \frac{1}{\sigma} \exp \left(-\frac{y_{t}-\mathbf{x}_{t} \beta}{\sigma}\right) \exp \left(-\exp \left(-\frac{y_{t}-\mathbf{x}_{t} \beta}{\sigma}\right)\right), \quad y_{t} \in \mathbb{R} .
$$

Seja

$$
y_{t}^{\prime}=d y_{t}+\mathbf{x}_{t} \mathbf{u}, \quad \beta^{\prime}=d \beta+\mathrm{u} \quad \text { e } \quad \sigma^{\prime}=d \sigma,
$$

$t=1, \ldots, n$, onde $d$ é um escalar positivo e u é um vetor de dimensão $k$. Seja $y^{\prime}=\left(y_{1}^{\prime}, \ldots, y_{n}^{\prime}\right)^{\top}$. Temos 
que

$$
L_{y}(\beta, \sigma)=d^{n} L_{y^{\prime}}\left(\beta^{\prime}, \sigma^{\prime}\right)
$$

Se $L_{y}(\beta, \sigma)$ é maximizada em

$$
\widehat{\beta}=\widehat{\beta}\left(y_{1}, \ldots, y_{n}\right) \quad \text { e } \quad \widehat{\sigma}=\widehat{\sigma}\left(y_{1}, \ldots, y_{n}\right)
$$

então $L_{y^{\prime}}\left(\beta^{\prime}, \sigma^{\prime}\right)$ é maximizada em

$$
\widehat{\beta}\left(y_{1}^{\prime}, \ldots, y_{n}^{\prime}\right)=d \widehat{\beta}+\mathrm{u} \quad \text { e } \quad \widehat{\sigma}\left(y_{1}^{\prime}, \ldots, y_{n}^{\prime}\right)=d \widehat{\sigma}
$$

Se $d=1 / \sigma$ e u $=-(1 / \sigma) \beta$ temos que $y^{\prime}=(y-\mu) / \sigma=z$. Assim,

$$
\widehat{\beta}\left(z_{1}, \ldots, z_{n}\right)=\frac{\widehat{\beta}-\beta}{\sigma} \text { e } \widehat{\sigma}\left(z_{1}, \ldots, z_{n}\right)=\frac{\widehat{\sigma}}{\sigma} .
$$

Então $(\widehat{\beta}-\beta) / \sigma$ e $\widehat{\sigma} / \sigma$ são funções de $z_{1}, \ldots, z_{n}$, cuja distribuição conjunta não depende dos parâmetros desconhecidos. Portanto, pelo Resultado 1 , podemos concluir que as distribuições de $(\widehat{\beta}-\beta) / \sigma$ e $\widehat{\sigma} / \sigma$ não dependem dos parâmetros desconhecidos, isto é, são quantidades pivotais.

Resultado 3. Sob as condições definidas acima, $(\widehat{\nu}-\nu) / \sigma, \widehat{\sigma} / \sigma,\left(\tilde{\nu}^{c}-\nu^{c}\right) / \sigma$ e $\tilde{\sigma} / \sigma$ são quantidades pivotais.

Demonstração.

$$
\begin{aligned}
L_{y}\left(\nu, \nu^{c}, \sigma\right) & =\prod_{t=1}^{n} f\left(y_{t} ; \nu, \nu^{c}, \sigma\right) \\
& =\prod_{t=1}^{n} \frac{1}{\sigma} \exp \left(-\frac{y_{t}-\mathbf{x}_{t}\left(\nu^{\top}, \nu^{\top c}\right)^{\top}}{\sigma}\right) \exp \left(-\exp \left(-\frac{\left.y_{t}-\mathbf{x}_{t} \nu^{\top}, \nu^{\top c}\right)^{\top}}{\sigma}\right)\right), \quad y_{t} \in \mathbb{R} .
\end{aligned}
$$

Seja

$$
y_{t}^{\prime}=d y_{t}+\mathbf{x}_{t}\left(\mathrm{u}^{\top}, \mathrm{u}^{c \top}\right)^{\top}, \quad \nu^{\prime}=d \nu+\mathrm{u}, \quad \nu^{c \prime}=d \nu^{c}+\mathrm{u}^{c} \quad \text { e } \quad \sigma^{\prime}=d \sigma
$$

$t=1, \ldots, n$, onde $d$ é um escalar positivo e u é um vetor de dimensão $r$ e u é um vetor de dimensão $k-r$. Seja $y^{\prime}=\left(y_{1}^{\prime}, \ldots, y_{n}^{\prime}\right)^{\top}$. Temos que

$$
L_{y}\left(\nu, \nu^{c}, \sigma\right)=d^{n} L_{y^{\prime}}\left(\nu^{\prime}, \nu^{c \prime}, \sigma^{\prime}\right)
$$

Se $L_{y}\left(\nu, \nu^{c}, \sigma\right)$ é maximizada em

$$
\widehat{\nu}=\widehat{\nu}\left(y_{1}, \ldots, y_{n}\right), \quad \widehat{\nu^{c}}=\widehat{\nu^{c}}\left(y_{1}, \ldots, y_{n}\right) \quad \text { e } \quad \widehat{\sigma}=\widehat{\sigma}\left(y_{1}, \ldots, y_{n}\right)
$$

então $L_{y^{\prime}}\left(\nu^{\prime}, \nu^{c \prime}, \sigma^{\prime}\right)$ é maximizada em

$$
\widehat{\nu}\left(y_{1}^{\prime}, \ldots, y_{n}^{\prime}\right)=d \widehat{\nu}+\mathrm{u}, \quad \widehat{\nu^{c}}\left(y_{1}^{\prime}, \ldots, y_{n}^{\prime}\right)=d \widehat{\nu^{c}}+\mathrm{u}^{c} \quad \text { e } \quad \widehat{\sigma}\left(y_{1}^{\prime}, \ldots, y_{n}^{\prime}\right)=d \widehat{\sigma}
$$


Se $d=1 / \sigma, \mathrm{u}=-(1 / \sigma) \nu$ e $\mathrm{u}^{c}=-(1 / \sigma) \nu^{c}$ temos que $y^{\prime}=(y-\mu) / \sigma=z$. Assim,

$$
\widehat{\nu}\left(z_{1}, \ldots, z_{n}\right)=\frac{\widehat{\nu}-\nu}{\sigma}, \quad \widehat{\nu^{c}}\left(z_{1}, \ldots, z_{n}\right)=\frac{\widehat{\nu^{c}}-\nu^{c}}{\sigma} \quad \text { e } \widehat{\sigma}\left(z_{1}, \ldots, z_{n}\right)=\frac{\widehat{\sigma}}{\sigma}
$$

Então $(\widehat{\nu}-\nu) / \sigma,\left(\widehat{\nu^{c}}-\nu^{c}\right) / \sigma$ e $\widehat{\sigma} / \sigma$ são funções de $z_{1}, \ldots, z_{n}$, cuja distribuição conjunta não depende dos parâmetros desconhecidos. Portanto, pelo Resultado 1, podemos concluir que as distribuições de $(\widehat{\nu}-\nu) / \sigma$, $\left(\widehat{\nu^{c}}-\nu^{c}\right) / \sigma$ e $\widehat{\sigma} / \sigma$ não dependem dos parâmetros desconhecidos, isto é, são quantidades pivotais.

Sob $\mathcal{H}_{0}: \nu=\nu_{0}$ onde $\nu_{0}$ é um vetor fixo de dimensão $r, L_{y}\left(\nu_{0}, \nu^{c}, \sigma\right)$ é maximizada em

$$
{\tilde{\nu^{c}}}^{c}=\tilde{\nu}^{c}\left(y_{1}, \ldots, y_{n}\right) \quad \text { e } \quad \tilde{\sigma}=\tilde{\sigma}\left(y_{1}, \ldots, y_{n}\right)
$$

então $L_{y^{\prime}}\left(\nu_{0}^{\prime}, \nu^{\prime c}, \sigma^{\prime}\right)$ é maximizada em

$$
\tilde{\nu^{c}}\left(y_{1}^{\prime}, \ldots, y_{n}^{\prime}\right)=d \tilde{\nu^{c}}+u^{c} \quad \text { e } \quad \tilde{\sigma}\left(y_{1}^{\prime}, \ldots, y_{n}^{\prime}\right)=d \tilde{\sigma}
$$

Se $d=1 / \sigma, \mathrm{u}=-(1 / \sigma) \nu$ e $\mathrm{u}^{c}=-(1 / \sigma) \nu^{c}$ temos que $y^{\prime}=(y-\mu) / \sigma=z$. Assim,

$$
\tilde{\nu^{c}}\left(z_{1}, \ldots, z_{n}\right)=\frac{\tilde{\nu^{c}}-\nu^{c}}{\sigma} \text { e } \quad \tilde{\sigma}\left(z_{1}, \ldots, z_{n}\right)=\frac{\tilde{\sigma}}{\sigma}
$$

Então $\left(\tilde{\nu}^{c}-\nu^{c}\right) / \sigma$ e $\tilde{\sigma} / \sigma$ são funções de $z_{1}, \ldots, z_{n}$, cuja distribuição conjunta não depende dos parâmetros desconhecidos. Portanto, pelo Resultado 1, podemos concluir que as distribuições de $\left(\tilde{\nu}^{c}-\nu^{c}\right) / \sigma$ e $\tilde{\sigma} / \sigma$ não dependem dos parâmetros desconhecidos, isto é, são quantidades pivotais.

As estatísticas da razão de verossimilhanças, Wald, escore e gradiente são dadas respectivamente por $w=2\left\{\ell(\widehat{\nu}, \widehat{\psi})-\ell\left(\nu_{0}, \widetilde{\psi}\right)\right\}, W=\left(\widehat{\nu}-\nu_{0}\right)^{\top}\left(\widehat{I}^{\nu \nu}\right)^{-1}\left(\widehat{\nu}-\nu_{0}\right), S_{R}=\widetilde{U}_{\nu}^{\top} \widetilde{I}^{\nu \nu} \widetilde{U}_{\nu}$ e $S_{T}=\widetilde{U}_{\nu}^{\top}\left(\widehat{\nu}-\nu_{0}\right)$, em que

$$
I^{-1}=\left[\begin{array}{ll}
I^{\nu \nu} & I^{\nu \psi} \\
I^{\psi \nu} & I^{\psi \psi}
\end{array}\right]
$$

é a inversa da informação de Fisher.

Resultado 4. Sob as condições definidas acima, a distribuição da estatística da razão de verossimilhanças não depende do parâmetro de dispersão $(\sigma) . \quad$ Da Silva et al. (2008, p. 806)

Se $\sigma_{t}=\sigma$ para $t=1, \ldots, n$ então $Z=\iota, \sigma=\sigma \mathcal{I}$ e $H=\mathcal{I}$ em que $\mathcal{I}$ é a matriz identidade. Se $\eta\left(x_{t}, \beta\right)=x_{t}^{\top} \beta$ então $X$, a matriz de derivadas de $\eta=\left(\eta_{1}, \ldots, \eta_{n}\right)^{\top}$ com respeito a $\beta^{\top}$, é constante. Se a função de ligação $g$ é a identidade então $T=\operatorname{diag}\left\{1 / g^{\prime}\left(\mu_{1}\right) \ldots, 1 / g^{\prime}\left(\mu_{n}\right)\right\}=\mathcal{I}$.

Resultado 5. Sob as condições definidas acima, a distribuição da estatística de Wald $(W)$ não depende do parâmetro de dispersão $(\sigma)$.

Demonstração. Sob as condições definidas acima, podemos escrever a informação de Fisher (2.14) como

$$
I=\frac{1}{\sigma^{2}}\left[\begin{array}{cc}
X^{\top} X & (\mathcal{E}-1) X^{\top} \iota \\
\left((\mathcal{E}-1) X^{\top} \iota\right)^{\top} & \left(1+\Gamma^{(2)}(2)\right) \iota^{\top} \iota
\end{array}\right],
$$


isto é, $I=1 / \sigma^{2} \mathcal{I}$ em que $\mathcal{I}$ é uma matriz constante. A inversa da informação de Fisher é $I^{-1}=\sigma^{2} \mathcal{I}^{-1}$. A estatística de Wald pode ser escrita como

$$
\begin{aligned}
W & =\left(\widehat{\nu}-\nu_{0}\right)^{\top}\left(\widehat{I}^{\nu \nu}\right)^{-1}\left(\widehat{\nu}-\nu_{0}\right)=\frac{\left(\widehat{\nu}-\nu_{0}\right)^{\top}}{\sigma} \sigma^{2}\left(\widehat{\sigma}^{2} \mathcal{I}^{\nu \nu}\right)^{-1} \frac{\left(\widehat{\nu}-\nu_{0}\right)}{\sigma} \\
& =\frac{\left(\widehat{\nu}-\nu_{0}\right)^{\top}}{\sigma}\left(\frac{\sigma}{\widehat{\sigma}}\right)^{2}\left(\mathcal{I}^{\nu \nu}\right)^{-1} \frac{\left(\widehat{\nu}-\nu_{0}\right)}{\sigma}
\end{aligned}
$$

Pelo Resultado 3, a estatística de Wald é um produto de funções de $z_{1}, \ldots, z_{n}$ e da matriz $\left(\mathcal{I}^{\nu \nu}\right)^{-1}$ constante. Logo, pelo Resultado 1 a distribuição de $W$ não depende de $\sigma$.

Resultado 6. Sob as condições definidas acima, a distribuição da estatística escore $S_{R}$ não depende do parâmetro de dispersão $(\sigma)$.

Demonstração. Sob as condições definidas acima, podemos escrever a função escore (6) como

$$
U=\frac{1}{\sigma}\left[\begin{array}{c}
X^{\top} T(\iota-e) \\
\iota^{\top}(\mathfrak{z}-\mathcal{Z} e-\iota)
\end{array}\right],
$$

isto é, $U=1 / \sigma \mathcal{U}$ em que $\mathcal{U}=\mathcal{U}\left(z_{1}, \ldots, z_{n}\right)$ é um vetor cuja distribuição não depende de $\sigma$, pelo Resultado 1 .

Temos que $\widetilde{\mathcal{U}}=\mathcal{U}\left(\widetilde{z}_{1}, \ldots, \widetilde{z}_{n}\right)$ e

$$
\begin{aligned}
\widetilde{z}_{t} & =\frac{y_{t}-\widetilde{\mu}_{t}}{\widetilde{\sigma}_{t}}=\frac{\sigma_{t}}{\widetilde{\sigma}_{t}} \frac{y_{t}-\mu_{t}+\mu_{t}-\widetilde{\mu}_{t}}{\sigma_{t}} \\
& =\frac{\sigma_{t}}{\widetilde{\sigma}_{t}}\left(\frac{y_{t}-\mu_{t}}{\sigma_{t}}+\frac{\mu_{t}-\widetilde{\mu}_{t}}{\sigma_{t}}\right)=\frac{\sigma_{t}}{\widetilde{\sigma}_{t}}\left(z_{t}+\frac{\mu_{t}-\widetilde{\mu}_{t}}{\sigma_{t}}\right) .
\end{aligned}
$$

isto é, $\widetilde{\mathcal{U}}=\mathcal{U}\left(\sigma_{t} / \widetilde{\sigma}_{t},\left(\mu_{t}-\widetilde{\mu}_{t}\right) / \sigma_{t}, z_{t}\right) \operatorname{com} t=1, \ldots, n$. A quantidade $\left(\mu_{t}-\widetilde{\mu}_{t}\right) / \sigma_{t}$ é função de $z_{1}, \ldots, z_{n}$, conforme Lawles (2003, p. 562), assim como $\sigma_{t} / \widetilde{\sigma}_{t}$ (Resultado 3). Logo a distribuição de $\widetilde{\mathcal{U}}$ é função de $z_{1}, \ldots, z_{n}$. A estatística escore é

$$
S_{R}=\widetilde{U}_{\nu}^{\top} \widetilde{I}^{\nu \nu} \widetilde{U}_{\nu}=\frac{1}{\widetilde{\sigma}} \widetilde{\mathcal{U}}_{\nu}^{\top} \widetilde{\sigma}^{2} \mathcal{I}^{\nu \nu} \frac{1}{\widetilde{\sigma}} \widetilde{\mathcal{U}}_{\nu}=\widetilde{\mathcal{U}}_{\nu}^{\top} \mathcal{I}^{\nu \nu} \widetilde{\mathcal{U}}_{\nu}
$$

lembrando que $I=1 / \sigma^{2} \mathcal{I}$ e $\mathcal{I}$ é constante, conforme a demonstração do Resultado 5. Logo a estatística escore é função de $z_{1}, \ldots, z_{n}$ e, pelo Resultado 1 , sua distribuição não depende de $\sigma$.

Resultado 7. Sob as condições definidas acima, a distribuição da estatística gradiente $S_{T}$ não depende do parâmetro de dispersão $(\sigma)$.

Demonstração. Sob as condições definidas acima, a estatística gradiente é

$$
S_{T}=\widetilde{U}_{\nu}^{\top}\left(\widehat{\nu}-\nu_{0}\right)=\frac{1}{\widetilde{\sigma}} \widetilde{\mathcal{U}}_{\nu}^{\top}\left(\widehat{\nu}-\nu_{0}\right)=\frac{\sigma}{\widetilde{\sigma}} \widetilde{\mathcal{U}}_{\nu}^{\top} \frac{\left(\widehat{\nu}-\nu_{0}\right)}{\sigma}
$$


lembrando que $U=1 / \sigma \mathcal{U}$ e $\widetilde{\mathcal{U}}=\widetilde{\mathcal{U}}\left(z_{1}, \ldots, z_{n}\right)$ conforme a demostração do Resultado 6. Logo $S_{T}$ é função $z_{1}, \ldots, z_{n}$ (ver Resultado 3). Logo, pelo Resultado1, a distribuição de $S_{T}$ não depende de $\sigma$.

Resultado 8. Sob as condições definidas acima, a distribuição da estatística de Skovgaard $w^{*}$ não depende do parâmetro de dispersão $(\sigma)$.

Demonstração. Sob as condições definidas acima, podemos escrever a informação observada (2.10) como

$$
\begin{gathered}
J_{\beta \beta}=\frac{1}{\sigma^{2}} X^{\top} \mathcal{Z}^{\dagger} X \\
J_{\beta \gamma}=J_{\gamma \beta}^{\top}=\frac{1}{\sigma^{2}} X^{\top}\left(\mathcal{I}-\mathcal{Z}^{\dagger}+\mathcal{Z} \mathcal{Z}^{\dagger}\right) \iota
\end{gathered}
$$

$\mathrm{e}$

$$
J_{\gamma \gamma}=\frac{1}{\sigma^{2}}\left(-\mathcal{I}+2 \mathcal{Z}-2 \mathcal{Z} \mathcal{Z}^{\dagger}+\mathcal{Z}^{2} \mathcal{Z}^{\dagger}\right) \iota
$$

isto é, $J=1 / \sigma^{2} \mathcal{J}$ em que $\mathcal{J}=\mathcal{J}\left(z_{1}, \ldots, z_{n}\right)$ é uma matriz cuja distribuição não depende de $\sigma$.

Temos

$$
\begin{aligned}
\bar{q} & =\left[\begin{array}{c}
\widehat{X}^{\top} \widehat{\sigma}^{-1} \widehat{T} C\left(\mathcal{I}-M D^{\dagger}\right) \iota \\
\widehat{Z}^{\top} \widehat{\sigma}^{-1} \widehat{H}\left(C\left(\mathcal{E} \mathcal{I}+N D^{\dagger}\right)-\mathcal{I}\right) \iota
\end{array}\right] \\
& =\left[\begin{array}{c}
\frac{1}{\widetilde{\sigma}} X^{\top}\left(\mathcal{I}-M D^{\dagger}\right) \iota \\
\frac{1}{\widehat{\sigma}} \iota^{\top}\left(\frac{\widehat{\sigma}}{\tilde{\sigma}}\left(\mathcal{E} \mathcal{I}+N D^{\dagger}\right)-\mathcal{I}\right) \iota
\end{array}\right]=\frac{1}{\widehat{\sigma}}\left[\begin{array}{c}
\frac{\widehat{\sigma}}{\sigma} \frac{\sigma}{\widetilde{\sigma}} X^{\top}\left(\mathcal{I}-M D^{\dagger}\right) \iota \\
\iota^{\top}\left(\frac{\widehat{\sigma}}{\sigma} \frac{\sigma}{\widetilde{\sigma}}\left(\mathcal{E} \mathcal{I}+N D^{\dagger}\right)-\mathcal{I}\right) \iota
\end{array}\right]
\end{aligned}
$$

e

$$
\begin{aligned}
\bar{\Upsilon} & =\left[\begin{array}{cc}
\widehat{X}^{\top} \widehat{\sigma}^{-1} \widehat{T} C M D^{\dagger} \widetilde{T} \widetilde{\sigma}^{-1} \widetilde{X} & \widehat{X}^{\top} \widehat{\sigma}^{-1} \widehat{T} C\left\{\mathrm{I}+D^{\dagger}(-M-C N+M D)\right\} \widetilde{H} \widetilde{\sigma}^{-1} \widetilde{Z} \\
-\widehat{Z}^{\top} \widehat{\sigma}^{-1} \widehat{H} C N D^{\dagger} \widetilde{T} \widetilde{\sigma}^{-1} \widetilde{X} & \widehat{Z}^{\top} \widehat{\sigma}^{-1} \widehat{H} C\left\{\mathcal{E} \mathrm{I}+D^{\dagger}(N+C P-N D)\right\} \widetilde{H} \widetilde{\sigma}^{-1} \widetilde{Z}
\end{array}\right] \\
= & {\left[\begin{array}{cc}
\frac{1}{\widehat{\sigma} \widetilde{\sigma}} X^{\top} M D^{\dagger} X & \frac{1}{\widehat{\sigma} \widetilde{\sigma}} X^{\top}\left\{\mathrm{I}+D^{\dagger}(-M-C N+M D)\right\} \iota \\
-\frac{1}{\hat{\sigma} \widetilde{\sigma}} \iota^{\top} N D^{\dagger} X & \frac{1}{\bar{\sigma} \widetilde{\sigma}} \iota^{\top}\left\{\mathcal{E} \mathrm{I}+D^{\dagger}(N+C P-N D)\right\} \iota
\end{array}\right] . }
\end{aligned}
$$

Lembrando que $C=\operatorname{diag}\left\{\widehat{\sigma}_{1} / \widetilde{\sigma}_{1}, \ldots, \widehat{\sigma}_{n} / \widetilde{\sigma}_{n}\right\}, D=\operatorname{diag}\left\{\left(\widehat{\mu}_{1}-\widetilde{\mu}_{1}\right) / \widetilde{\sigma}_{1}, \ldots,\left(\widehat{\mu}_{n}-\widetilde{\mu}_{n}\right) / \widetilde{\sigma}_{n}\right\}, D^{\dagger}=$ $\operatorname{diag}\left\{\exp \left(-\left(\widehat{\mu}_{1}-\widetilde{\mu}_{1}\right) / \widetilde{\sigma}_{1}\right), \ldots, \exp \left(-\left(\widehat{\mu}_{n}-\widetilde{\mu}_{n}\right) / \widetilde{\sigma}_{n}\right)\right\}, M=\operatorname{diag}\left\{\Gamma\left(1+\widehat{\sigma}_{1} / \widetilde{\sigma}_{1}\right), \ldots, \Gamma\left(1+\widehat{\sigma}_{n} / \widetilde{\sigma}_{n}\right)\right\}$, $N=\operatorname{diag}\left\{\Gamma^{(1)}\left(1+\widehat{\sigma}_{1} / \widetilde{\sigma}_{1}\right), \ldots, \Gamma^{(1)}\left(1+\widehat{\sigma}_{n} / \widetilde{\sigma}_{n}\right)\right\}, P=\operatorname{diag}\left\{\Gamma^{(2)}\left(1+\widehat{\sigma}_{1} / \widetilde{\sigma}_{1}\right), \ldots, \Gamma^{(2)}\left(1+\widehat{\sigma}_{n} / \widetilde{\sigma}_{n}\right)\right\} \mathrm{e}$

$$
\frac{\widehat{\mu}_{t}-\widetilde{\mu}_{t}}{\widetilde{\sigma}_{t}}=\left(\frac{\widehat{\mu}_{t}-\mu}{\sigma_{t}}+\frac{\mu-\widetilde{\mu}_{t}}{\sigma_{t}}\right) \frac{\sigma_{t}}{\widetilde{\sigma}_{t}}
$$

que é função de $z_{1}, \ldots, z_{n}$, segundo Lawles (2003, p. 562). Logo $\bar{q}=1 / \widehat{\sigma} \mathcal{Q}$, em que $\mathcal{Q}$ é um vetor função das quantidades $\widehat{\sigma} / \sigma$ e $\widetilde{\sigma} / \sigma$ então é função de $z_{1}, \ldots, z_{n}$ (ver Resultado 3 ). E ainda $\bar{\Upsilon}=1 /(\widehat{\sigma} \tilde{\sigma}) \mathcal{Y}$ em que $\mathcal{Y}=\mathcal{Y}\left(z_{1}, \ldots, z_{n}\right)$ é uma matriz função das quantidades $\widehat{\sigma} / \sigma, \widetilde{\sigma} / \sigma,\left(\widehat{\mu}_{t}-\mu\right) / \sigma$ e $\left(\widetilde{\mu}_{t}-\mu\right) / \sigma$ então é função de $z_{1}, \ldots, z_{n}$ (ver Resultado 3). 
Temos

$$
\xi=\frac{\left(|\widetilde{I}||\widehat{I}|\left|\widetilde{J}_{\psi \psi}\right|\right)^{1 / 2}}{|\bar{\Upsilon}|\left|\left[\widetilde{I} \widetilde{\Upsilon}^{-1} \widehat{J} \widehat{I}^{-1} \bar{\Upsilon}\right]_{\psi \psi}\right|^{1 / 2}} \frac{\left(\widetilde{U}^{\top} \bar{\Upsilon}^{-1} \widehat{I} \widehat{J}^{-1} \bar{\Upsilon} \widetilde{I}^{-1} \widetilde{U}\right)^{r / 2}}{w^{r / 2-1} \widetilde{U}^{\top} \bar{\Upsilon}^{-1} \bar{q}}
$$

e $U=1 / \sigma \mathcal{U}, J=1 / \sigma^{2} \mathcal{J}, I=1 / \sigma^{2} \mathcal{I}, \bar{q}=1 / \widehat{\sigma} \mathcal{Q}$ e $\bar{\Upsilon}=1 /(\widehat{\sigma} \tilde{\sigma}) \mathcal{Y} \log 0$

$$
\begin{aligned}
& \xi=\frac{\left(\left|1 / \tilde{\sigma}^{2} \widetilde{\mathcal{I}}\right|\left|1 / \widehat{\sigma}^{2} \widehat{\mathcal{I}}\right|\left|1 / \tilde{\sigma}^{2} \widetilde{\mathcal{J}}_{\psi \psi}\right|\right)^{1 / 2}}{|1 /(\hat{\sigma} \tilde{\sigma}) \mathcal{Y}|\left|\left[1 / \tilde{\sigma}^{2} \widetilde{\mathcal{I}}(1 /(\widehat{\sigma} \tilde{\sigma}) \mathcal{Y})^{-1} 1 / \widehat{\sigma}^{2} \widehat{\mathcal{J}} \widehat{\sigma}^{2} \widehat{\mathcal{I}}^{-1} 1 /(\widehat{\sigma} \tilde{\sigma}) \mathcal{Y}\right]_{\psi \psi}\right|^{1 / 2}} \times \\
& \frac{\left(1 / \tilde{\sigma} \widetilde{\mathcal{U}}^{\top}(1 /(\widehat{\sigma} \tilde{\sigma}) \mathcal{Y})^{-1} 1 / \widehat{\sigma}^{2} \widehat{\mathcal{I}}\left(1 / \widehat{\sigma}^{2} \widehat{\mathcal{J}}\right)^{-1} 1 /(\widehat{\sigma} \tilde{\sigma}) \mathcal{Y}\left(1 / \tilde{\sigma}^{2} \widetilde{\mathcal{I}}\right)^{-1} 1 / \tilde{\sigma} \widetilde{\mathcal{U}}\right)^{r / 2}}{w^{r / 2-1} 1 / \tilde{\sigma} \widetilde{\mathcal{U}}^{\top}(1 /(\widehat{\sigma} \tilde{\sigma}) \mathcal{Y})^{-1} 1 / \widehat{\sigma} \mathcal{Q}} \\
& =\frac{\left(n / \tilde{\sigma}^{2}|\widetilde{\mathcal{I}}| n / \widehat{\sigma}^{2}|\widehat{\mathcal{I}}| s / \tilde{\sigma}^{2}\left|\widetilde{\mathcal{J}}_{\psi \psi}\right|\right)^{1 / 2}}{n /(\widehat{\sigma} \tilde{\sigma})|\mathcal{Y}|\left|\left[1 / \tilde{\sigma} \tilde{\mathcal{I}}^{2} \hat{\sigma} \tilde{\sigma} \mathcal{Y}^{-1} 1 / \widehat{\sigma}^{2} \widehat{\mathcal{J}} \widehat{\sigma}^{2} \widehat{\mathcal{I}}^{-1} 1 /(\widehat{\sigma} \tilde{\sigma}) \mathcal{Y}\right]_{\psi \psi}\right|^{1 / 2}} \times \\
& \frac{\left(1 / \tilde{\sigma} \widetilde{\mathcal{U}}^{\top} \widehat{\sigma} \tilde{\sigma} \mathcal{Y}^{-1} 1 / \widehat{\sigma}^{2} \widehat{\mathcal{I}} \widehat{\sigma}^{2} \widehat{\mathcal{J}}^{-1} 1 /(\widehat{\sigma} \tilde{\sigma}) \mathcal{Y} \tilde{\sigma}^{2} \widetilde{\mathcal{I}}^{-1} 1 / \tilde{\sigma} \tilde{\mathcal{U}}\right)^{r / 2}}{w^{r / 2-1} 1 / \tilde{\sigma} \widetilde{\mathcal{U}}^{\top} \widehat{\sigma} \tilde{\sigma} \mathcal{Y}^{-1} 1 / \widehat{\sigma} \mathcal{Q}} \\
& =\frac{n /\left(\tilde{\sigma}^{2} \widehat{\sigma}\right)\left(s|\widetilde{\mathcal{I}}||\widehat{\mathcal{I}}|\left|\widetilde{\mathcal{J}}_{\psi \psi}\right|\right)^{1 / 2}}{n /(\widehat{\sigma} \tilde{\sigma})|\mathcal{Y}|\left|1 / \tilde{\sigma}^{2}\left[\widetilde{\mathcal{I}} \mathcal{Y}^{-1} \widehat{\mathcal{J}} \widehat{\mathcal{I}}^{-1} \mathcal{Y}\right]_{\psi \psi}\right|^{1 / 2}} \frac{\left(\tilde{\mathcal{U}}^{\top} \mathcal{Y}^{-1} \widehat{\mathcal{I}} \widehat{\mathcal{J}}^{-1} \mathcal{Y} \widetilde{\mathcal{I}}^{-1} \widetilde{\mathcal{U}}\right)^{r / 2}}{w^{r / 2-1} \widetilde{\mathcal{U}}^{\top} \mathcal{Y}^{-1} \mathcal{Q}} \\
& =\frac{1 / \tilde{\sigma}\left(s|\widetilde{\mathcal{I}}| \widehat{\mathcal{I}}|| \widetilde{\mathcal{J}}_{\psi \psi} \mid\right)^{1 / 2}}{|\mathcal{Y}|\left(s / \tilde{\sigma}^{2}\right)^{1 / 2}\left|\left[\widetilde{\mathcal{I}} \mathcal{Y}^{-1} \widehat{\mathcal{J}} \widehat{\mathcal{I}}^{-1} \mathcal{Y}\right]_{\psi \psi}\right|^{1 / 2}} \frac{\left(\widetilde{\mathcal{U}}^{\top} \mathcal{Y}^{-1} \widehat{\mathcal{I}} \widehat{\mathcal{J}}^{-1} \mathcal{Y} \widetilde{\mathcal{I}}^{-1} \widetilde{\mathcal{U}}\right)^{r / 2}}{w^{r / 2-1} \widetilde{\mathcal{U}}^{\top} \mathcal{Y}^{-1} \mathcal{Q}} \\
& =\frac{\left(|\widetilde{\mathcal{I}}||\widehat{\mathcal{I}}|\left|\widetilde{\mathcal{J}}_{\psi \psi}\right|\right)^{1 / 2}}{|\mathcal{Y}|\left|\left[\widetilde{\mathcal{I}} \mathcal{Y}^{-1} \widehat{\mathcal{J}} \hat{\mathcal{I}}^{-1} \mathcal{Y}\right]_{\psi \psi}\right|^{1 / 2}} \frac{\left(\widetilde{\mathcal{U}}^{\top} \mathcal{Y}^{-1} \widehat{\mathcal{I}} \widehat{\mathcal{J}}^{-1} \mathcal{Y} \widetilde{\mathcal{I}}^{-1} \widetilde{\mathcal{U}}\right)^{r / 2}}{w^{r / 2-1} \widetilde{\mathcal{U}}^{\top} \mathcal{Y}^{-1} \mathcal{Q}}
\end{aligned}
$$

que é função de $z_{1}, \ldots, z_{n}$. Logo, pelo Resultado 1 , a distribuição de $w^{*}$ não depende de $\sigma$.

Resultado 9. Sob as condições definidas acima, $\left(y_{t}-x_{t} \widehat{\beta}\right) / \widehat{\sigma}$ é uma estatística ancilar.

Demonstração. Temos que

$$
\frac{y_{t}-x_{t} \widehat{\beta}}{\widehat{\sigma}}=\frac{\sigma}{\widehat{\sigma}}\left(\frac{y_{t}-x_{t} \beta}{\sigma}\right)+\frac{\sigma}{\widehat{\sigma}}\left(\frac{x_{t} \beta-x_{t} \widehat{\beta}}{\sigma}\right)=\frac{\sigma}{\widehat{\sigma}}\left(\frac{y_{t}-x_{t} \beta}{\sigma}\right)+\frac{\sigma}{\widehat{\sigma}} x_{t}\left(\frac{\beta-\widehat{\beta}}{\sigma}\right) .
$$

Pelos Resultados 1 e 2 , a estatística $\left(y_{t}-x_{t} \widehat{\beta}\right) / \widehat{\sigma}$ é função de $z_{1}, \ldots, z_{n}$, cuja distribuição conjunta não depende dos parâmetros desconhecidos, logo sua distribuição não depende de parâmetros, isto é, é uma estatística ancilar. 


\section{Apêndice E}

\section{Ajustes da razão de verossimilhanças sinalizada}

Ajustes da razão de verossimilhanças sinalizada:

\section{E.1 Barndorff-Nielsen (1986)}

Substituindo $y_{t}$ por $\widehat{\sigma}_{t} a_{t}+\widehat{\mu}_{t}$ em (2.5) temos que

$$
\begin{gathered}
\ell_{t}(\theta ; \widehat{\theta}, a)=-\ln \sigma_{t}-\frac{\widehat{\sigma}_{t} a_{t}+\widehat{\mu}_{t}-\mu_{t}}{\sigma_{t}}-\exp \left(-\frac{\widehat{\sigma}_{t} a_{t}+\widehat{\mu}_{t}-\mu_{t}}{\sigma_{t}}\right) . \\
\frac{\partial \ell_{t}(\theta ; \widehat{\theta}, a)}{\partial \widehat{\mu}_{t}}=-\frac{1}{\sigma_{t}}-\exp \left(-\frac{\widehat{\sigma}_{t} a_{t}+\widehat{\mu}_{t}-\mu_{t}}{\sigma_{t}}\right)\left(-\frac{1}{\sigma_{t}}\right)=\frac{1}{\sigma_{t}}\left(-1+\exp \left(-\frac{y_{t}-\mu_{t}}{\sigma_{t}}\right)\right) \\
\frac{\partial \ell_{t}(\theta ; \widehat{\theta}, a)}{\partial \widehat{\sigma}_{t}}=-\frac{a_{t}}{\sigma_{t}}-\exp \left(-\frac{\widehat{\sigma}_{t} a_{t}+\widehat{\mu}_{t}-\mu_{t}}{\sigma_{t}}\right)\left(-\frac{a_{t}}{\sigma_{t}}\right)=\frac{a_{t}}{\sigma_{t}}\left(-1+\exp \left(-\frac{y_{t}-\mu_{t}}{\sigma_{t}}\right)\right)
\end{gathered}
$$

Por (A.1)

$$
\begin{gathered}
\frac{\partial^{2} \ell_{t}(\theta ; \widehat{\theta}, a)}{\partial \mu_{t} \partial \widehat{\mu}_{t}}=-\frac{1}{\sigma_{t}} \exp \left(-\frac{\widehat{\sigma}_{t} a_{t}+\widehat{\mu}_{t}-\mu_{t}}{\sigma_{t}}\right)\left(-\frac{1}{\sigma_{t}}\right) \\
=\frac{1}{\sigma_{t} 2} \exp \left(-\frac{\widehat{\sigma}_{t} a_{t}+\widehat{\mu}_{t}-\mu_{t}}{\sigma_{t}}\right)=\frac{1}{\sigma_{t}^{2}} \exp \left(-\frac{y_{t}-\mu_{t}}{\sigma_{t}}\right) \\
\frac{\partial^{2} \ell(\theta ; \widehat{\theta}, a)}{\partial \mu_{t} \partial \widehat{\sigma}_{t}}=\frac{\partial}{\partial \widehat{\sigma}_{t}}\left(\frac{1}{\sigma_{t}}-\frac{1}{\sigma_{t}} \exp \left(-\frac{\widehat{\sigma}_{t} a_{t}+\widehat{\mu}_{t}-\mu_{t}}{\sigma_{t}}\right)\right)=-\frac{1}{\sigma_{t}} \exp \left(-\frac{\widehat{\sigma}_{t} a_{t}+\widehat{\mu}_{t}-\mu_{t}}{\sigma_{t}}\right)\left(-\frac{a_{t}}{\sigma_{t}}\right) \\
=\frac{a_{t}}{\sigma_{t}^{2}} \exp \left(-\frac{\widehat{\sigma}_{t} a_{t}+\widehat{\mu}_{t}-\mu_{t}}{\sigma_{t}}\right)=\frac{a_{t}}{\sigma_{t}^{2}} \exp \left(-\frac{y_{t}-\mu_{t}}{\sigma_{t}}\right)
\end{gathered}
$$

Por (A.3)

$$
\begin{aligned}
& \frac{\partial^{2} \ell(\theta ; \widehat{\theta}, a)}{\partial \sigma_{t} \partial \widehat{\mu}_{t}} \\
= & \frac{\partial}{\partial \widehat{\mu}_{t}}\left(\frac{1}{\sigma_{t}}\left(-1+\frac{\widehat{\sigma}_{t} a_{t}+\widehat{\mu}_{t}-\mu_{t}}{\sigma_{t}}-\exp \left(-\frac{\widehat{\sigma}_{t} a_{t}+\widehat{\mu}_{t}-\mu_{t}}{\sigma_{t}}\right)\left(\frac{\widehat{\sigma}_{t} a_{t}+\widehat{\mu}_{t}-\mu_{t}}{\sigma_{t}}\right)\right)\right)
\end{aligned}
$$




$$
\begin{aligned}
= & \frac{1}{\sigma_{t}}\left(\frac{1}{\sigma_{t}}-\frac{1}{\sigma_{t}} \exp \left(-\frac{\widehat{\sigma}_{t} a_{t}+\widehat{\mu}_{t}-\mu_{t}}{\sigma_{t}}\right)-\frac{\widehat{\sigma}_{t} a_{t}+\widehat{\mu}_{t}-\mu_{t}}{\sigma_{t}} \exp \left(-\frac{\widehat{\sigma}_{t} a_{t}+\widehat{\mu}_{t}-\mu_{t}}{\sigma_{t}}\right)\left(-\frac{1}{\sigma_{t}}\right)\right) \\
= & \frac{1}{\sigma_{t}^{2}}\left(1-\exp \left(-\frac{y_{t}-\mu_{t}}{\sigma_{t}}\right)+\frac{y_{t}-\mu_{t}}{\sigma_{t}} \exp \left(-\frac{y_{t}-\mu_{t}}{\sigma_{t}}\right)\right) \\
& \frac{\partial^{2} \ell(\theta ; \widehat{\theta}, a)}{\partial \sigma_{t} \partial \widehat{\sigma}_{t}} \\
= & \frac{\partial}{\partial \widehat{\sigma}_{t}}\left(\frac{1}{\sigma_{t}}\left(-1+\frac{\widehat{\sigma}_{t} a_{t}+\widehat{\mu}_{t}-\mu_{t}}{\sigma_{t}}-\exp \left(-\frac{\widehat{\sigma}_{t} a_{t}+\widehat{\mu}_{t}-\mu_{t}}{\sigma_{t}}\right)\left(\frac{\widehat{\sigma}_{t} a_{t}+\widehat{\mu}_{t}-\mu_{t}}{\sigma_{t}}\right)\right)\right) \\
= & \frac{1}{\sigma_{t}}\left(\frac{a_{t}}{\sigma_{t}}-\frac{a_{t}}{\sigma_{t}} \exp \left(-\frac{\widehat{\sigma}_{t} a_{t}+\widehat{\mu}_{t}-\mu_{t}}{\sigma_{t}}\right)-\left(\frac{\widehat{\sigma}_{t} a_{t}+\widehat{\mu}_{t}-\mu_{t}}{\sigma_{t}}\right) \exp \left(-\frac{\widehat{\sigma}_{t} a_{t}+\widehat{\mu}_{t}-\mu_{t}}{\sigma_{t}}\right)\left(-\frac{a_{t}}{\sigma_{t}}\right)\right) \\
= & \frac{a_{t}}{\sigma_{t}^{2}}\left(1-\exp \left(-\frac{y_{t}-\mu_{t}}{\sigma_{t}}\right)+\left(\frac{y_{t}-\mu_{t}}{\sigma_{t}}\right) \exp \left(-\frac{y_{t}-\mu_{t}}{\sigma_{t}}\right)\right) \cdot
\end{aligned}
$$

Temos que

$$
\frac{\partial \ell(\theta ; \widehat{\theta}, a)}{\partial \widehat{\theta}}=\left(\frac{\partial \ell(\theta ; \widehat{\theta}, a)}{\partial \widehat{\beta}} \quad \frac{\partial \ell(\theta ; \widehat{\theta}, a)}{\partial \widehat{\gamma}}\right)
$$

Por (E.2)

$$
\frac{\partial \ell(\theta ; \widehat{\theta}, a)}{\partial \widehat{\beta}_{i}}=\sum_{t=1}^{n} \frac{\partial \ell_{t}(\theta ; \widehat{\theta}, a)}{\partial \widehat{\mu}_{t}} \frac{\partial \widehat{\mu}_{t}}{\partial \widehat{\eta}_{t}} \frac{\partial \widehat{\eta}_{t}}{\partial \widehat{\beta}_{i}}=\sum_{t=1}^{n} \frac{1}{\sigma_{t}}\left(-1+\exp \left(-\frac{y_{t}-\mu_{t}}{\sigma_{t}}\right)\right) \frac{\partial \widehat{\mu}_{t}}{\partial \widehat{\eta}_{t}} \frac{\partial \widehat{\eta}_{t}}{\partial \widehat{\beta}_{i}}
$$

e

$$
\frac{\partial \ell(\theta ; \widehat{\theta}, a)}{\partial \widehat{\beta}}=\iota^{\top} \sigma^{-1}\left(-\mathcal{I}+\mathcal{Z}^{\dagger}\right) \widehat{T} \widehat{X}
$$

Por (E.3)

$$
\frac{\partial \ell(\theta ; \widehat{\theta}, a)}{\partial \widehat{\gamma}_{i}}=\sum_{t=1}^{n} \frac{\partial \ell_{t}(\theta ; \widehat{\theta}, a)}{\partial \widehat{\sigma}} \frac{\partial \widehat{\sigma}_{t}}{\partial \widehat{\delta}_{t}} \frac{\partial \widehat{\delta}_{t}}{\partial \widehat{\gamma}_{i}}=\sum_{t=1}^{n} \frac{a_{t}}{\sigma_{t}}\left(-1+\exp \left(-\frac{y_{t}-\mu_{t}}{\sigma_{t}}\right)\right) \frac{\partial \widehat{\sigma}_{t}}{\partial \widehat{\delta}_{t}} \frac{\partial \widehat{\delta}_{t}}{\partial \widehat{\gamma}_{i}}
$$

e

$$
\frac{\partial \ell(\theta ; \widehat{\theta}, a)}{\partial \widehat{\gamma}}=\iota^{\top} \widehat{\mathcal{Z}} \sigma^{-1}\left(-\mathcal{I}+\mathcal{Z}^{\dagger}\right) \widehat{H} \widehat{Z}
$$

Então

$$
\begin{aligned}
& \frac{\partial \ell(\theta ; \widehat{\theta}, a)}{\partial \widehat{\theta}}=\iota^{\top} \sigma^{-1}\left(-\mathcal{I}+\mathcal{Z}^{\dagger}\right)(\widehat{T} \widehat{X} \quad \widehat{\mathcal{Z}} \widehat{H} \widehat{Z}), \\
& l_{; \widehat{\theta}}(\widehat{\theta})=\left.\frac{\partial \ell(\theta ; \widehat{\theta}, a)}{\partial \widehat{\theta}}\right|_{\theta=\widehat{\theta}}=\iota^{\top} \widehat{\sigma}^{-1}\left(-\mathcal{I}+\widehat{\mathcal{Z}}^{\dagger}\right)(\widehat{T} \widehat{X} \quad \widehat{\mathcal{Z}} \widehat{H} \widehat{Z}), \\
& l_{; \widehat{\theta}}(\widetilde{\theta})=\left.\frac{\partial \ell(\theta ; \widehat{\theta}, a)}{\partial \widehat{\theta}}\right|_{\theta=\widetilde{\theta}}=\iota^{\top} \widetilde{\sigma}^{-1}\left(-\mathcal{I}+\widetilde{\mathcal{Z}}^{\dagger}\right)(\widehat{T} \widehat{X} \quad \widehat{\mathcal{Z}} \widehat{H} \widehat{Z})
\end{aligned}
$$

e

$$
l_{; \widehat{\theta}}(\widehat{\theta})-l_{; \widehat{\theta}}(\widetilde{\theta})=\iota^{\top}\left(\widehat{\sigma}^{-1}\left(-\mathcal{I}+\widehat{\mathcal{Z}}^{\dagger}\right)-\widetilde{\sigma}^{-1}\left(-\mathcal{I}+\widetilde{\mathcal{Z}}^{\dagger}\right)\right)(\widehat{T} \widehat{X} \quad \widehat{\mathcal{Z}} \widehat{H} \widehat{Z})
$$


Temos que

$$
\frac{\partial^{2} \ell(\theta ; \widehat{\theta}, a)}{\partial \theta \partial \widehat{\theta}}=\left(\begin{array}{ll}
\frac{\partial^{2} \ell(\theta ; \widehat{\theta}, a)}{\partial \beta \partial \widehat{\beta}} & \frac{\partial^{2} \ell(\theta ; \widehat{\theta}, a)}{\partial \beta \partial \widehat{\gamma}} \\
\frac{\partial^{2} \ell(\theta ; \widehat{\theta}, a)}{\partial \gamma \partial \widehat{\beta}} & \frac{\partial^{2} \ell(\theta ; \widehat{\theta}, a)}{\partial \gamma \partial \widehat{\gamma}}
\end{array}\right)
$$

Por (E.4)

$$
\begin{aligned}
\frac{\partial^{2} \ell(\theta ; \widehat{\theta}, a)}{\partial \beta_{i} \partial \widehat{\beta}_{j}} & =\sum_{t=1}^{n} \frac{\partial}{\partial \widehat{\beta}_{j}}\left(\frac{\partial \ell_{t}(\theta ; \widehat{\theta}, a)}{\partial \beta_{i}}\right)=\sum_{t=1}^{n} \frac{\partial}{\partial \widehat{\beta}_{j}}\left(\frac{\partial \ell_{t}(\theta ; \widehat{\theta}, a)}{\partial \mu_{t}} \frac{\partial \mu_{t}}{\partial \eta_{t}} \frac{\partial \eta_{t}}{\partial \beta_{i}}\right) \\
& =\sum_{t=1}^{n}\left(\frac{\partial \ell_{t}(\theta ; \widehat{\theta}, a)}{\partial \mu_{t} \partial \widehat{\mu}_{t}} \frac{\partial \widehat{\mu}_{t}}{\partial \widehat{\eta}_{t}} \frac{\partial \widehat{\eta}_{t}}{\partial \widehat{\beta}_{j}}\right) \frac{\partial \mu_{t}}{\partial \eta_{t}} \frac{\partial \eta_{t}}{\partial \beta_{i}} \\
& =\sum_{t=1}^{n}\left(\frac{1}{\sigma_{t}^{2}} \exp \left(-\frac{y_{t}-\mu_{t}}{\sigma_{t}}\right) \frac{\partial \widehat{\mu}_{t}}{\partial \widehat{\eta}_{t}} \frac{\partial \widehat{\eta}_{t}}{\partial \widehat{\beta}_{j}}\right) \frac{\partial \mu_{t}}{\partial \eta_{t}} \frac{\partial \eta_{t}}{\partial \beta_{i}}
\end{aligned}
$$

$\mathrm{e}$

$$
\frac{\partial^{2} \ell(\theta ; \widehat{\theta}, a)}{\partial \beta \partial \widehat{\beta}}=X^{\top} \mathcal{Z}^{\dagger} T \sigma^{-2} \widehat{T} \widehat{X}
$$

Por (E.5)

$$
\begin{aligned}
\frac{\partial^{2} \ell(\theta ; \widehat{\theta}, a)}{\partial \beta_{i} \partial \widehat{\gamma}_{j}} & =\sum_{t=1}^{n} \frac{\partial}{\partial \widehat{\gamma}_{j}}\left(\frac{\partial \ell_{t}(\theta ; \widehat{\theta}, a)}{\partial \beta_{i}}\right)=\sum_{t=1}^{n} \frac{\partial}{\partial \widehat{\gamma}_{j}}\left(\frac{\partial \ell_{t}(\theta ; \widehat{\theta}, a)}{\partial \mu_{t}} \frac{\partial \mu_{t}}{\partial \eta_{t}} \frac{\partial \eta_{t}}{\partial \beta_{i}}\right) \\
& =\sum_{t=1}^{n}\left(\frac{\partial^{2} \ell_{t}(\theta ; \widehat{\theta}, a)}{\partial \mu_{t} \partial \widehat{\sigma}} \frac{\partial \widehat{\sigma}}{\partial \widehat{\delta}_{t}} \frac{\partial \widehat{\delta}_{t}}{\partial \widehat{\gamma}_{j}}\right) \frac{\partial \mu_{t}}{\partial \eta_{t}} \frac{\partial \eta_{t}}{\partial \beta_{i}} \\
& =\sum_{t=1}^{n}\left(\frac{a_{t}}{\sigma_{t}^{2}} \exp \left(-\frac{y_{t}-\mu_{t}}{\sigma_{t}}\right) \frac{\partial \widehat{\sigma}}{\partial \widehat{\delta}_{t}} \frac{\partial \widehat{\delta}_{t}}{\partial \widehat{\gamma}_{j}}\right) \frac{\partial \mu_{t}}{\partial \eta_{t}} \frac{\partial \eta_{t}}{\partial \beta_{i}}
\end{aligned}
$$

e

$$
\frac{\partial^{2} \ell(\theta ; \widehat{\theta}, a)}{\partial \beta \partial \widehat{\gamma}}=X^{\top} \mathcal{Z}^{\dagger} T \sigma^{-2} \widehat{\mathcal{Z}} \widehat{H} \widehat{Z}
$$

Por (E.6)

$$
\begin{aligned}
\frac{\partial^{2} \ell(\theta ; \widehat{\theta}, a)}{\partial \gamma_{i} \partial \widehat{\beta}_{j}} & =\sum_{t=1}^{n} \frac{\partial}{\partial \widehat{\beta}_{j}}\left(\frac{\partial \ell_{t}(\theta ; \widehat{\theta}, a)}{\partial \gamma_{i}}\right)=\sum_{t=1}^{n} \frac{\partial}{\partial \widehat{\beta}_{j}}\left(\frac{\partial \ell_{t}(\theta ; \widehat{\theta}, a)}{\partial \sigma} \frac{\partial \sigma_{t}}{\partial \delta_{t}} \frac{\partial \delta_{t}}{\partial \gamma_{i}}\right) \\
& =\sum_{t=1}^{n}\left(\frac{\partial \ell_{t}(\theta ; \widehat{\theta}, a)}{\partial \sigma \partial \widehat{\mu}_{t}} \frac{\partial \widehat{\mu}_{t}}{\partial \widehat{\eta}_{t}} \frac{\partial \widehat{\eta}_{t}}{\partial \widehat{\beta}_{j}}\right) \frac{\partial \sigma_{t}}{\partial \delta_{t}} \frac{\partial \delta_{t}}{\partial \gamma_{i}} \\
& =\sum_{t=1}^{n}\left(\frac{1}{\sigma_{t}^{2}}\left(1-\exp \left(-\frac{y_{t}-\mu_{t}}{\sigma_{t}}\right)+\frac{y_{t}-\mu_{t}}{\sigma_{t}} \exp \left(-\frac{y_{t}-\mu_{t}}{\sigma_{t}}\right)\right) \frac{\partial \widehat{\mu}_{t}}{\partial \widehat{\eta}_{t}} \frac{\partial \widehat{\eta}_{t}}{\partial \widehat{\beta}_{j}}\right) \frac{\partial \sigma_{t}}{\partial \delta_{t}} \frac{\partial \delta_{t}}{\partial \gamma_{i}}
\end{aligned}
$$

e

$$
\frac{\partial^{2} \ell(\theta ; \widehat{\theta}, a)}{\partial \gamma \partial \widehat{\beta}}=Z^{\top}\left(\mathcal{I}-\mathcal{Z}^{\dagger}+\mathcal{Z} \mathcal{Z}^{\dagger}\right) H \sigma^{-2} \widehat{T} \widehat{X}
$$


Por (E.7)

$$
\begin{aligned}
\frac{\partial^{2} \ell(\theta ; \widehat{\theta}, a)}{\partial \gamma_{i} \partial \widehat{\gamma}_{j}} & =\sum_{t=1}^{n} \frac{\partial}{\partial \widehat{\gamma}_{j}}\left(\frac{\partial \ell_{t}(\theta ; \widehat{\theta}, a)}{\partial \gamma_{i}}\right)=\sum_{t=1}^{n} \frac{\partial}{\partial \widehat{\gamma}_{j}}\left(\frac{\partial \ell_{t}(\theta ; \widehat{\theta}, a)}{\partial \sigma} \frac{\partial \sigma_{t}}{\partial \delta_{t}} \frac{\partial \delta_{t}}{\partial \gamma_{i}}\right) \\
& =\sum_{t=1}^{n}\left(\frac{\partial \ell_{t}(\theta ; \widehat{\theta}, a)}{\partial \sigma \partial \widehat{\sigma}} \frac{\partial \widehat{\sigma}}{\partial \widehat{\delta}_{t}} \frac{\partial \widehat{\delta}_{t}}{\partial \widehat{\gamma}_{j}}\right) \frac{\partial \sigma_{t}}{\partial \delta_{t}} \frac{\partial \delta_{t}}{\partial \gamma_{i}} \\
& =\sum_{t=1}^{n}\left(\frac{a_{t}}{\sigma_{t}^{2}}\left(1-\exp \left(-\frac{y_{t}-\mu_{t}}{\sigma_{t}}\right)+\left(\frac{y_{t}-\mu_{t}}{\sigma_{t}}\right) \exp \left(-\frac{y_{t}-\mu_{t}}{\sigma_{t}}\right)\right) \frac{\partial \widehat{\sigma}}{\partial \widehat{\delta}_{t}} \frac{\partial \widehat{\delta}_{t}}{\partial \widehat{\gamma}_{j}}\right) \frac{\partial \sigma_{t}}{\partial \delta_{t}} \frac{\partial \delta_{t}}{\partial \gamma_{i}}
\end{aligned}
$$

e

$$
\frac{\partial^{2} \ell(\theta ; \widehat{\theta}, a)}{\partial \gamma \partial \widehat{\gamma}}=Z^{\top}\left(\mathcal{I}-\mathcal{Z}^{\dagger}+\mathcal{Z} \mathcal{Z}^{\dagger}\right) H \sigma^{-2} \widehat{\mathcal{Z}} \widehat{H} \widehat{Z}
$$

Então

$$
\frac{\partial^{2} \ell(\theta ; \widehat{\theta}, a)}{\partial \theta \partial \widehat{\theta}}=\left(\begin{array}{c}
X^{\top} \mathcal{Z}^{\dagger} T \sigma^{-2} \\
Z^{\top}\left(\mathcal{I}-\mathcal{Z}^{\dagger}+\mathcal{Z} \mathcal{Z}^{\dagger}\right) H \sigma^{-2}
\end{array}\right)\left(\begin{array}{ll}
\widehat{T} \widehat{X} & \widehat{\mathcal{Z}} \widehat{H} \widehat{Z}
\end{array}\right)
$$

Lembrando que no modelo de regressão linear temos que $Z=\iota, \sigma=\operatorname{diag}\{\sigma, \ldots, \sigma\}, g$ e $h$ são a função identidade, logo, temos que $T=\mathcal{I}$ e $H=\mathcal{I}$. Assim podemos escrever

$$
l_{; \widehat{\theta}}(\widehat{\theta})-l_{; \widehat{\theta}}(\widetilde{\theta})=\iota^{\top}\left(\widehat{\sigma}^{-1}\left(-\mathcal{I}+\widehat{\mathcal{Z}}^{\dagger}\right)-\widetilde{\sigma}^{-1}\left(-\mathcal{I}+\widetilde{\mathcal{Z}}^{\dagger}\right)\right)(\widehat{X} \quad \widehat{\mathcal{Z}} \iota)
$$

e

$$
\frac{\partial^{2} \ell(\theta ; \widehat{\theta}, a)}{\partial \theta \partial \widehat{\theta}}=\sigma^{-2}\left(\begin{array}{c}
X^{\top} \mathcal{Z}^{\dagger} \\
\iota^{\top}\left(\mathcal{I}-\mathcal{Z}^{\dagger}+\mathcal{Z} \mathcal{Z}^{\dagger}\right)
\end{array}\right)\left(\begin{array}{ll}
\widehat{X} & \widehat{\mathcal{Z}} \iota
\end{array}\right)
$$

\section{E.2 DiCiccio \& Martin (1993)}

Temos que

$$
\begin{gathered}
\frac{\partial^{2} \mu_{t}}{\partial \eta_{t} \partial \sigma_{t}}=\frac{\partial^{2} \sigma_{t}}{\partial \delta_{t} \partial \mu_{t}}=0 \\
\frac{\partial \ell(\vartheta)}{\partial \beta_{r}}=\frac{\partial \ell\left(\beta_{r}, \kappa, \tau\right)}{\partial \beta_{r}}=\sum_{t=1}^{n} \frac{\partial \ell_{t}\left(\mu_{t}, \sigma_{t}\right)}{\partial \beta_{r}}=\sum_{t=1}^{n}\left\{\frac{\partial \ell_{t}\left(\mu_{t}, \sigma_{t}\right)}{\partial \mu_{t}} \frac{\partial \mu_{t}}{\partial \eta_{t}} \frac{\partial \eta_{t}}{\partial \beta_{r}}+\frac{\partial \ell_{t}\left(\mu_{t}, \sigma_{t}\right)}{\partial \sigma_{t}} \frac{\partial \sigma_{t}}{\partial \delta_{t}} \frac{\partial \delta_{t}}{\partial \beta_{r}}\right\}, \\
\frac{\partial^{2} \ell\left(\beta_{r}, \kappa, \tau\right)}{\partial^{2} \beta_{r}}=\sum_{t=1}^{n}\left\{\frac{\partial^{2} \ell_{t}\left(\mu_{t}, \sigma_{t}\right)}{\partial \beta_{r}^{2}}\right\}=\sum_{t=1}^{n}\left\{\left(\frac{\partial^{2} \ell_{t}\left(\mu_{t}, \sigma_{t}\right)}{\partial \mu_{t}^{2}}\left(\frac{\partial \mu_{t}}{\partial \eta_{t}}\right)^{2}+\frac{\partial \ell_{t}\left(\mu_{t}, \sigma_{t}\right)}{\partial \mu_{t}} \frac{\partial^{2} \mu_{t}}{\partial \eta_{t}^{2}}\right)\left(\frac{\partial \eta_{t}}{\partial \beta_{r}}\right)^{2}\right. \\
+2 \frac{\partial^{2} \ell_{t}\left(\mu_{t}, \sigma_{t}\right)}{\partial \mu_{t} \partial \sigma_{t}} \frac{\partial \sigma_{t}}{\partial \delta_{t}} \frac{\partial \delta_{t}}{\partial \beta_{r}} \frac{\partial \mu_{t}}{\partial \eta_{t}} \frac{\partial \eta_{t}}{\partial \beta_{r}}+\frac{\partial \ell_{t}\left(\mu_{t}, \sigma_{t}\right)}{\partial \mu_{t}} \frac{\partial \mu_{t}}{\partial \eta_{t}} \frac{\partial^{2} \eta_{t}}{\partial \beta_{r}^{2}} \\
\left.+\left(\frac{\partial^{2} \ell_{t}\left(\mu_{t}, \sigma_{t}\right)}{\partial \sigma_{t}^{2}}\left(\frac{\partial \sigma_{t}}{\partial \delta_{t}}\right)^{2}+\frac{\partial \ell_{t}\left(\mu_{t}, \sigma_{t}\right)}{\partial \sigma_{t}} \frac{\partial^{2} \sigma_{t}}{\partial \delta_{t}^{2}}\right)\left(\frac{\partial \delta_{t}}{\partial \beta_{r}}\right)^{2}+\frac{\partial \ell_{t}\left(\mu_{t}, \sigma_{t}\right)}{\partial \sigma_{t}} \frac{\partial \sigma_{t}}{\partial \delta_{t}} \frac{\partial^{2} \delta_{t}}{\partial \beta_{r}^{2}}\right\}
\end{gathered}
$$




$$
\begin{gathered}
\frac{\partial^{2} \ell\left(\beta_{r}, \kappa, \tau\right)}{\partial \beta_{r} \partial \kappa_{i}}=\sum_{t=1}^{n}\left\{\frac{\partial^{2} \ell_{t}\left(\mu_{t}, \sigma_{t}\right)}{\partial \beta_{r} \partial \kappa_{i}}\right\}=\sum_{t=1}^{n}\left\{\left[\frac{\partial^{2} \ell_{t}\left(\mu_{t}, \sigma_{t}\right)}{\partial \mu_{t}^{2}}\left(\frac{\partial \mu_{t}}{\partial \eta_{t}}\right)^{2}+\frac{\partial \ell_{t}\left(\mu_{t}, \sigma_{t}\right)}{\partial \mu_{t}} \frac{\partial^{2} \mu_{t}}{\partial \eta_{t}^{2}}\right] \frac{\partial \eta_{t}}{\partial \kappa_{i}} \frac{\partial \eta_{t}}{\partial \beta_{r}}\right. \\
\left.+\frac{\partial \ell_{t}\left(\mu_{t}, \sigma_{t}\right)}{\partial \mu_{t}} \frac{\partial \mu_{t}}{\partial \eta_{t}} \frac{\partial^{2} \eta_{t}}{\partial \beta_{r} \partial \kappa_{i}}+\frac{\partial^{2} \ell_{t}\left(\mu_{t}, \sigma_{t}\right)}{\partial \sigma_{t} \partial \mu_{t}} \frac{\partial \mu_{t}}{\partial \eta_{t}} \frac{\partial \sigma_{t}}{\partial \delta_{t}} \frac{\partial \eta_{t}}{\partial \kappa_{i}} \frac{\partial \delta_{t}}{\partial \beta_{r}}+\frac{\partial \ell_{t}\left(\mu_{t}, \sigma_{t}\right)}{\partial \sigma_{t}} \frac{\partial \sigma_{t}}{\partial \delta_{t}} \frac{\partial^{2} \delta_{t}}{\partial \beta_{r} \partial \kappa_{i}}\right\} \quad \text { (E.11) } \\
\frac{\partial^{2} \ell\left(\beta_{r}, \kappa, \tau\right)}{\partial \beta_{r} \partial \tau_{i}}=\sum_{t=1}^{n}\left\{\frac{\partial^{2} \ell_{t}\left(\mu_{t}, \sigma_{t}\right)}{\partial \beta_{r} \partial \tau_{i}}\right\}=\sum_{t=1}^{n}\left\{\frac{\partial^{2} \ell_{t}\left(\mu_{t}, \sigma_{t}\right)}{\partial \mu_{t} \partial \sigma} \frac{\partial \sigma_{t}}{\partial \delta_{t}} \frac{\partial \mu_{t}}{\partial \eta_{t}} \frac{\partial \delta_{t}}{\partial \tau_{i}} \frac{\partial \eta_{t}}{\partial \beta_{r}}+\frac{\partial \ell_{t}\left(\mu_{t}, \sigma_{t}\right)}{\partial \mu_{t}} \frac{\partial \mu_{t}}{\partial \eta_{t}} \frac{\partial^{2} \eta_{t}}{\partial \beta_{r} \partial \tau_{i}}\right. \\
\left.+\left[\frac{\partial^{2} \ell_{t}\left(\mu_{t}, \sigma_{t}\right)}{\partial \sigma_{t}^{2}}\left(\frac{\partial \sigma_{t}}{\partial \delta_{t}}\right)^{2}+\frac{\partial \ell_{t}\left(\mu_{t}, \sigma_{t}\right)}{\partial \sigma_{t}} \frac{\partial^{2} \sigma_{t}}{\partial \delta_{t}^{2}}\right] \frac{\partial \delta_{t}}{\partial \tau_{i}} \frac{\partial \delta_{t}}{\partial \beta_{r}}+\frac{\partial \ell_{t}\left(\mu_{t}, \sigma_{t}\right)}{\partial \sigma_{t}} \frac{\partial \sigma_{t}}{\partial \delta_{t}} \frac{\partial^{2} \delta_{t}}{\partial \beta_{r} \partial \tau_{i}}\right\}
\end{gathered}
$$

Considerando o modelo linear (2.1) com componentes sistemáticos dados por

$$
g\left(\mu_{t}\right)=\eta_{t}=\eta\left(x_{t}, \beta\right)=\mathbf{x}_{t} \cdot \beta, \quad h\left(\sigma_{t}\right)=\delta_{t}=\delta\left(z_{t}, \gamma\right)=\mathbf{z}_{t} \cdot \gamma
$$

$\eta_{t}^{*}(\vartheta)=\left(x_{t r}-\mathbf{x}_{t(r)} A^{\top}\right) \beta_{r}+\mathbf{x}_{t(r)} \kappa \mathrm{e} \delta_{t}^{*}(\vartheta)=\mathbf{z}_{t} \cdot \tau-\beta_{r} \mathbf{z}_{t} \cdot B^{\top}$, segue que

$$
\frac{\partial \eta_{t}^{*}}{\partial \tau_{i}}=\frac{\partial \delta_{t}^{*}}{\partial \kappa_{i}}=\frac{\partial^{2} \eta_{t}^{*}}{\partial \beta_{r}^{2}}=\frac{\partial^{2} \eta_{t}^{*}}{\partial \beta_{r} \partial \kappa_{i}}=\frac{\partial^{2} \eta_{t}^{*}}{\partial \beta_{r} \partial \tau_{i}}=\frac{\partial^{2} \delta_{t}^{*}}{\partial \beta_{r}^{2}}=\frac{\partial^{2} \delta_{t}^{*}}{\partial \beta_{r} \partial \kappa_{i}}=\frac{\partial^{2} \delta_{t}^{*}}{\partial \beta_{r} \partial \tau_{i}}=0
$$

Por (A.1), (A.3), (E.8), (E.9), (3.4) e (3.5)

$$
\begin{aligned}
\frac{\partial \ell^{*}\left(\beta_{r}, \kappa, \tau\right)}{\partial \beta_{r}}= & \sum_{t=1}^{n}\left\{\left(\frac{1}{\sigma_{t}}-\frac{1}{\sigma_{t}} \exp \left(-\frac{y_{t}-\mu_{t}}{\sigma_{t}}\right)\right)\left(\frac{1}{g^{\prime}\left(\mu_{t}\right)}\right)\left(x_{t r}-\mathbf{x}_{t(r)} A^{\top}\right)\right. \\
& \left.+\left(\frac{1}{\sigma_{t}}\left(-1+\frac{y_{t}-\mu_{t}}{\sigma_{t}}-\exp \left(-\frac{y_{t}-\mu_{t}}{\sigma_{t}}\right)\left(\frac{y_{t}-\mu_{t}}{\sigma_{t}}\right)\right)\right)\left(\frac{1}{h^{\prime}\left(\sigma_{t}\right)}\right)\left(-\mathbf{z}_{t} \cdot B^{\top}\right)\right\}
\end{aligned}
$$

tem a seguinte forma matricial

$$
\ell_{\beta_{r}}^{*}=\iota^{\top}\left(\sigma^{-1} T\left(\mathcal{I}-\mathcal{Z}^{\dagger}\right)\left(\mathbf{x}_{\cdot r}-X_{(r)} A^{\top}\right)-\sigma^{-1} H\left(-\mathcal{I}+\mathcal{Z}-\mathcal{Z} \mathcal{Z}^{\dagger}\right) Z B^{\top}\right) .
$$

Por (A.1), (A.2), (A.3), (A.4), (A.5), (A.6), (A.7), (E.8), (E.10), (E.13), (3.4) e (3.5) temos que

$$
\begin{aligned}
& \frac{\partial^{2} \ell^{*}\left(\beta_{r}, \kappa, \tau\right)}{\partial^{2} \beta_{r}}=\sum_{t=1}^{n}\{ \\
& {\left[\left(-\frac{1}{\sigma_{t}^{2}} \exp \left(-\frac{y_{t}-\mu_{t}}{\sigma_{t}}\right)\right)\left(\frac{1}{g^{\prime}\left(\mu_{t}\right)}\right)^{2}+\left(\frac{1}{\sigma_{t}}-\frac{1}{\sigma_{t}} \exp \left(-\frac{y_{t}-\mu_{t}}{\sigma_{t}}\right)\right)\left(-\frac{g^{\prime \prime}\left(\mu_{t}\right)}{g^{\prime}\left(\mu_{t}\right)^{3}}\right)\right]} \\
& \left(x_{t r}-\mathbf{x}_{t(r)} A^{\top}\right)^{2} \\
& -2 \frac{1}{\sigma_{t}^{2}}\left(1-\exp \left(-\frac{y_{t}-\mu_{t}}{\sigma_{t}}\right)+\left(\frac{y_{t}-\mu_{t}}{\sigma_{t}}\right) \exp \left(-\frac{y_{t}-\mu_{t}}{\sigma_{t}}\right)\right)\left(\frac{1}{g^{\prime}\left(\mu_{t}\right)}\right)\left(\frac{1}{h^{\prime}\left(\sigma_{t}\right)}\right) \\
& \left(x_{t r}-\mathbf{x}_{t(r)} A^{\top}\right)\left(-\mathbf{z}_{t} \cdot B^{\top}\right)
\end{aligned}
$$




$$
\begin{aligned}
& +\left[\frac{1}{\sigma_{t}^{2}}\left(1-2\left(\frac{y_{t}-\mu_{t}}{\sigma_{t}}\right)+2 \exp \left(-\frac{y_{t}-\mu_{t}}{\sigma_{t}}\right)\left(\frac{y_{t}-\mu_{t}}{\sigma_{t}}\right)-\exp \left(-\frac{y_{t}-\mu_{t}}{\sigma_{t}}\right)\left(\frac{y_{t}-\mu_{t}}{\sigma_{t}}\right)^{2}\right)\right. \\
& \left(\frac{1}{h^{\prime}\left(\sigma_{t}\right)}\right)^{2} \\
& \left.\left.+\frac{1}{\sigma_{t}}\left(-1+\frac{y_{t}-\mu_{t}}{\sigma_{t}}-\exp \left(-\frac{y_{t}-\mu_{t}}{\sigma_{t}}\right)\left(\frac{y_{t}-\mu_{t}}{\sigma_{t}}\right)\right)\left(-\frac{h^{\prime \prime}\left(\sigma_{t}\right)}{h^{\prime}\left(\sigma_{t}\right)^{3}}\right)\right]\left(-\mathbf{z}_{t} \cdot B^{\top}\right)^{2}\right\} .
\end{aligned}
$$

Logo, na forma matricial,

$$
\begin{aligned}
J_{\beta_{r} \beta_{r}}^{*}= & -\frac{\partial^{2} \ell\left(\beta_{r}, \kappa, \tau\right)}{\partial^{2} \beta_{r}} \\
= & \left(\mathbf{x} \cdot r-X_{(r)}^{\top} A^{\top}\right)^{\top} \sigma^{-1} T\left(\mathcal{Z}^{\dagger} \sigma^{-1}+\left(\mathcal{I}-\mathcal{Z}^{\dagger}\right) S T\right) T\left(\mathbf{x} \cdot r-X_{(r)}^{\top} A^{\top}\right) \\
& +2\left(\mathbf{x}_{\cdot r}-X_{(r)}^{\top} A^{\top}\right)^{\top} \sigma^{-1} T\left(\mathcal{I}-\mathcal{Z}^{\dagger}+\mathcal{Z} \mathcal{Z}^{\dagger}\right) H \sigma^{-1}\left(-Z B^{\top}\right) \\
& +\left(-Z B^{\top}\right)^{\top} \sigma^{-1} H\left(\left(-\mathcal{I}+2 \mathcal{Z}-2 \mathcal{Z} \mathcal{Z}^{\dagger}+\mathcal{Z}^{2} \mathcal{Z}^{\dagger}\right) \sigma^{-1}+\left(-\mathcal{I}+\mathcal{Z}-\mathcal{Z} \mathcal{Z}^{\dagger}\right) Q H\right) H\left(-Z B^{\top}\right)
\end{aligned}
$$

Por (A.1), (A.2), (A.5), (A.6), (E.8), (E.11), (E.13), (3.4) e (3.5) temos que

$$
\begin{aligned}
& \frac{\partial^{2} \ell^{*}\left(\beta_{r}, \kappa, \tau\right)}{\partial \beta_{r} \partial \kappa}=\sum_{t=1}^{n}\left\{\left(-\frac{1}{\sigma_{t}^{2}} \exp \left(-\frac{y_{t}-\mu_{t}}{\sigma_{t}}\right)\right)\left(\frac{1}{g^{\prime}\left(\mu_{t}\right)}\right)+\left(\frac{1}{\sigma_{t}}-\frac{1}{\sigma_{t}} \exp \left(-\frac{y_{t}-\mu_{t}}{\sigma_{t}}\right)\right)\right. \\
& \left(-\frac{g^{\prime \prime}\left(\mu_{t}\right)}{g^{\prime}\left(\mu_{t}\right)^{2}}\right)\left(\frac{1}{g^{\prime}\left(\mu_{t}\right)}\right)\left(x_{t r}-\mathbf{x}_{t(r)} A^{\top}\right) x_{t i} \\
& \left.-\frac{1}{\sigma_{t}^{2}}\left(1-\exp \left(-\frac{y_{t}-\mu_{t}}{\sigma_{t}}\right)+\left(\frac{y_{t}-\mu_{t}}{\sigma_{t}}\right) \exp \left(-\frac{y_{t}-\mu_{t}}{\sigma_{t}}\right)\right)\left(\frac{1}{g^{\prime}\left(\mu_{t}\right)}\right)\left(\frac{1}{h^{\prime}\left(\sigma_{t}\right)}\right) x_{t i}\left(-\mathbf{z}_{t} \cdot B^{\top}\right)\right\} .
\end{aligned}
$$

Logo, na forma matricial,

$$
\begin{aligned}
J_{\beta_{r} \kappa}^{*}=J_{\kappa \beta_{r}}^{* \top}=-\frac{\partial^{2} \ell\left(\beta_{r}, \kappa, \tau\right)}{\partial \beta_{r} \partial \kappa}= & \left(\mathbf{x}_{\cdot r}-X_{(r)} A^{\top}\right)^{\top} \sigma^{-1} T\left(\mathcal{Z}^{\dagger} \sigma^{-1}+\left(\mathcal{I}-\mathcal{Z}^{\dagger}\right) S T\right) T X_{(r)} \\
& +\left(-Z B^{\top}\right)^{\top} \sigma^{-1} T\left(\mathcal{I}-\mathcal{Z}^{\dagger}+\mathcal{Z} \mathcal{Z}^{\dagger}\right) H \sigma^{-1} X_{(r)} .
\end{aligned}
$$

Por (A.3), (A.4), (A.5), (A.7), (E.8), (E.12), (E.13), (3.4) e (3.5) temos que

$$
\begin{aligned}
& \frac{\partial^{2} \ell^{*}\left(\beta_{r}, \kappa, \tau\right)}{\partial \beta_{r} \partial \tau}=\sum_{t=1}^{n}\left\{-\frac{1}{\sigma_{t}^{2}}\left(1-\exp \left(-\frac{y_{t}-\mu_{t}}{\sigma_{t}}\right)+\left(\frac{y_{t}-\mu_{t}}{\sigma_{t}}\right) \exp \left(-\frac{y_{t}-\mu_{t}}{\sigma_{t}}\right)\right)\right. \\
& \left(\frac{1}{g^{\prime}\left(\mu_{t}\right)}\right)\left(\frac{1}{h^{\prime}\left(\sigma_{t}\right)}\right) z_{t i}\left(x_{t r}-\mathbf{x}_{t(r)} A^{\top}\right) \\
& +\left(\frac{1}{\sigma_{t}^{2}}\left(1-2\left(\frac{y_{t}-\mu_{t}}{\sigma_{t}}\right)+2 \exp \left(-\frac{y_{t}-\mu_{t}}{\sigma_{t}}\right)\left(\frac{y_{t}-\mu_{t}}{\sigma_{t}}\right)-\exp \left(-\frac{y_{t}-\mu_{t}}{\sigma_{t}}\right)\left(\frac{y_{t}-\mu_{t}}{\sigma_{t}}\right)^{2}\right)\right. \\
& \left(\frac{1}{h^{\prime}\left(\sigma_{t}\right)}\right)^{2}
\end{aligned}
$$




$$
\left.\left.+\frac{1}{\sigma_{t}}\left(-1+\frac{y_{t}-\mu_{t}}{\sigma_{t}}-\exp \left(-\frac{y_{t}-\mu_{t}}{\sigma_{t}}\right)\left(\frac{y_{t}-\mu_{t}}{\sigma_{t}}\right)\right)\left(-\frac{h^{\prime \prime}\left(\sigma_{t}\right)}{h^{\prime}\left(\sigma_{t}\right)^{3}}\right)\right)\left(-\mathbf{z}_{t} \cdot B^{\top}\right) z_{t i}\right\}
$$

Logo, na forma matricial,

$$
\begin{aligned}
J_{\beta_{r} \tau}^{*}=J_{\tau \beta_{r}}^{* \top}= & -\frac{\partial^{2} \ell\left(\beta_{r}, \kappa, \tau\right)}{\partial \beta_{r} \partial \tau}=\left(\mathbf{x}_{\cdot r}-X_{(r)} A^{\top}\right)^{\top} \sigma^{-1} T\left(\mathcal{I}-\mathcal{Z}^{\dagger}+\mathcal{Z} \mathcal{Z}^{\dagger}\right) H \sigma^{-1} Z \\
& +\left(-Z B^{\top}\right)^{\top} \sigma^{-1} H\left(\left(-\mathcal{I}+2 \mathcal{Z}-2 \mathcal{Z} \mathcal{Z}^{\dagger}+\mathcal{Z}^{2} \mathcal{Z}^{\dagger}\right) \sigma^{-1}+\left(-\mathcal{I}+\mathcal{Z}-\mathcal{Z} \mathcal{Z}^{\dagger}\right) Q H\right) H Z .
\end{aligned}
$$

Analogamente,

$$
\begin{aligned}
& J_{\kappa \kappa}^{*}=X_{(r)}^{\top} \sigma^{-1} T\left(\mathcal{Z}^{\dagger} \sigma^{-1}+\left(\mathcal{I}-\mathcal{Z}^{\dagger}\right) S T\right) T X_{(r)}, \quad J_{\kappa \tau}^{*}=J_{\tau \kappa}^{* \top}=X_{(r)}^{\top} \sigma^{-1} T\left(\mathcal{I}-\mathcal{Z}^{\dagger}+\mathcal{Z Z}^{\dagger}\right) H \sigma^{-1} Z, \\
& J_{\tau \tau}^{*}=Z^{\top} \sigma^{-1} H\left(\left(-\mathcal{I}+2 \mathcal{Z}-2 \mathcal{Z} \mathcal{Z}^{\dagger}+\mathcal{Z}^{2} \mathcal{Z}^{\dagger}\right) \sigma^{-1}+\left(-\mathcal{I}+\mathcal{Z}-\mathcal{Z Z}^{\dagger}\right) Q H\right) H Z, \\
& I_{\beta_{r} \beta_{r}=}\left(\mathbf{x} \cdot r-X_{(r)} A^{\top}\right)^{\top} \sigma^{-1} T^{2} \sigma^{-1}\left(\mathbf{x} \cdot r-X_{(r)} A^{\top}\right)-2(\mathcal{E}-1)\left(\mathbf{x} \cdot r-X_{(r)} A^{\top}\right)^{\top} \sigma^{-1} T H \sigma^{-1} Z B^{\top} \\
&+\left(1+\Gamma^{(2)}(2)\right)\left(Z B^{\top}\right)^{\top} \sigma^{-1} H^{2} \sigma^{-1} Z B^{\top} .
\end{aligned}
$$

Podemos escrever

$$
\begin{gathered}
\ell_{\beta_{r}}^{*}=\iota^{\top}\left(\sigma^{-1} T\left(\mathcal{I}-\mathcal{Z}^{\dagger}\right) \mathbf{v}_{1}+\sigma^{-1} H\left(-\mathcal{I}+\mathcal{Z}-\mathcal{Z}^{\dagger}\right) \mathbf{v}_{2}\right), \\
J_{\beta_{r} \beta_{r}}^{*}=\mathbf{v}_{1}^{\top} V_{\beta \beta} \mathbf{v}_{1}+2 \mathbf{v}_{1}^{\top} V_{\beta \gamma} \mathbf{v}_{2}+\mathbf{v}_{2}^{\top} V_{\gamma \gamma} \mathbf{v}_{2}, \quad J_{\beta_{r} \kappa}^{*}=J_{\kappa \beta_{r}}^{* \top}=\mathbf{v}_{1}^{\top} V_{\beta \beta} X_{(r)}+\mathbf{v}_{2}^{\top} V_{\beta \gamma} X_{(r)}, \\
J_{\beta_{r} \tau}^{*}=J_{\tau \beta_{r}}^{* \top}=\mathbf{v}_{1}^{\top} V_{\beta \gamma} Z+\mathbf{v}_{2}^{\top} V_{\gamma \gamma} Z, \quad J_{\kappa \kappa}^{*}=X_{(r)}^{\top} V_{\beta \beta} X_{(r)}, \\
J_{\kappa \tau}^{*}=J_{\tau \kappa}^{* \top}=X_{(r)}^{\top} V_{\beta \gamma} Z, \quad J_{\tau \tau}^{*}=Z^{\top} V_{\gamma \gamma} Z
\end{gathered}
$$

e

$$
I_{\beta_{r} \beta_{r}}^{*}=\mathbf{v}_{1}^{\top} \sigma^{-1} T^{2} \sigma^{-1} \mathbf{v}_{1}+2(\mathcal{E}-1) \mathbf{v}_{1}^{\top} \sigma^{-1} T H \sigma^{-1} \mathbf{v}_{2}+\left(1+\Gamma^{(2)}(2)\right) \mathbf{v}_{2}^{\top} \sigma^{-1} H^{2} \sigma^{-1} \mathbf{v}_{2} .
$$

Considerando o modelo não linear (2.1) homoscedástico com componentes sistemáticos da locação e dispersão dados, respectivamente, por

$$
\begin{gathered}
g\left(\mu_{t}\right)=\mu_{t}=\eta_{t}=\eta\left(x_{t}, \beta\right)=\beta_{0}+\beta_{1} x_{t 1}+\exp \left(\beta_{2} x_{t 2}\right), \\
h\left(\sigma_{t}\right)=\sigma_{t}=\delta_{t}=\delta\left(z_{t}, \gamma\right)=\gamma_{0} .
\end{gathered}
$$

Sendo $\eta_{t}^{*}(\vartheta)=\mathbf{x}_{t(2)}\left(\kappa-\beta_{2} A^{\top}\right)+\exp \left(\beta_{2} x_{t 2}\right)=\mathbf{x}_{t(2)} \kappa-\beta_{2} \mathbf{x}_{t(2)} A^{\top}+\exp \left(\beta_{2} x_{t 2}\right)$ e $\delta_{t}^{*}(\vartheta)=\mathbf{z}_{t} \cdot(\tau-$ $\left.\beta_{2} B^{\top}\right)=\mathbf{z}_{t} \cdot \tau-\beta_{2} \mathbf{z}_{t} \cdot B^{\top}$, segue que

$$
\frac{\partial \eta_{t}^{*}}{\partial \tau_{i}}=\frac{\partial \delta_{t}^{*}}{\partial \kappa_{i}}=\frac{\partial^{2} \eta_{t}^{*}}{\partial \beta_{2} \partial \kappa_{i}}=\frac{\partial^{2} \eta_{t}^{*}}{\partial \beta_{2} \partial \tau_{i}}=\frac{\partial^{2} \delta_{t}^{*}}{\partial \beta_{2}^{2}}=\frac{\partial^{2} \delta_{t}^{*}}{\partial \beta_{2} \partial \kappa_{i}}=\frac{\partial^{2} \delta_{t}^{*}}{\partial \beta_{2} \partial \tau_{i}}=0
$$


Por (A.1), (A.2), (A.3), (A.4), (A.5), (A.6), (A.7), (E.8), (E.14), (E.10), (3.6), (3.7) e (3.8) temos que

$$
J_{\beta_{r} \beta_{r}}^{*}=\mathbf{v}_{1}^{\top} V_{\beta \beta} \mathbf{v}_{1}^{\top}+2 \mathbf{v}_{1}^{\top} V_{\beta \gamma} \mathbf{v}_{2}-\iota^{\top}\left(\mathcal{I}-\mathcal{Z}^{\dagger}\right) T \sigma^{-1} \mathbf{v}_{3}+\mathbf{v}_{2}^{\top} V_{\gamma \gamma} \mathbf{v}_{2}
$$

em que $\mathbf{v}_{1}=\left(x_{12} \exp \left(\beta_{2} x_{12}\right)-\mathbf{x}_{1(2)} A^{\top}, \ldots, x_{n 2} \exp \left(\beta_{2} x_{n 2}\right)-\mathbf{x}_{n(2)} A^{\top}\right)^{\top}, \mathbf{v}_{2}=-Z B^{\top}, \mathbf{v}_{3}=$ $\left(x_{12}^{2} \exp \left(\beta_{2} x_{12}\right), \ldots, x_{n 2}^{2} \exp \left(\beta_{2} x_{n 2}\right)\right)^{\top}$ e $Z=\iota$.

\section{E.3 Severini (1999)}

Temos, por (2.5), (A.1) e (A.3), que

$$
\begin{aligned}
& \widehat{q}=\sum_{t=1}^{n}\left(\ell_{t}(\widehat{\theta})-\ell_{t}(\widetilde{\theta})\right) \frac{\partial \ell_{t}(\widehat{\theta})}{\partial \theta^{\top}}=\left(\begin{array}{c}
\sum_{t=1}^{n}\left(\ell_{t}(\widehat{\theta})-\ell_{t}(\widetilde{\theta})\right) \frac{\partial \ell_{t}(\widehat{\theta})}{\partial \mu_{t}} \frac{\partial \mu_{t}(\widehat{\theta})}{\partial \eta_{t}} \frac{\partial \eta_{t}(\widehat{\theta})}{\partial \beta_{1}} \\
\ldots \\
\sum_{t=1}^{n}\left(\ell_{t}(\widehat{\theta})-\ell_{t}(\widetilde{\theta})\right) \frac{\partial \ell_{t}(\widehat{\theta})}{\partial \mu_{t}} \frac{\partial \mu_{t}(\widehat{\theta})}{\partial \eta_{t}} \frac{\partial \eta_{t}(\widehat{\theta})}{\partial \beta_{k}} \\
\sum_{t=1}^{n}\left(\ell_{t}(\widehat{\theta})-\ell_{t}(\widetilde{\theta})\right) \frac{\partial \ell_{t}(\widehat{\theta})}{\partial \sigma_{t}} \frac{\partial \sigma_{t}(\widehat{\theta})}{\partial \delta_{t}} \frac{\partial \delta_{t}(\widehat{\theta})}{\partial \gamma_{1}} \\
\ldots \\
\sum_{t=1}^{n}\left(\ell_{t}(\widehat{\theta})-\ell_{t}(\widetilde{\theta})\right) \frac{\partial \ell_{t}(\widehat{\theta})}{\partial \sigma_{t}} \frac{\partial \sigma_{t}(\widehat{\theta})}{\partial \delta_{t}} \frac{\partial \delta_{t}(\widehat{\theta})}{\partial \gamma_{m}}
\end{array}\right) \\
& =\left(\begin{array}{c}
\widehat{X} \widehat{\sigma}^{-1} \widehat{T}(\widehat{\mathfrak{L}}-\widetilde{\mathfrak{L}})\left(\iota-\widehat{\mathfrak{z}}^{\dagger}\right) \\
\widehat{Z} \widehat{\sigma}^{-1} \widehat{H}(\widehat{\mathfrak{L}}-\widetilde{\mathfrak{L}})\left(-\iota+\mathfrak{z}-\mathcal{Z}_{\mathfrak{z}}^{\dagger}\right)
\end{array}\right)
\end{aligned}
$$

e

$$
\begin{aligned}
& \widehat{\Upsilon}=\sum_{t=1}^{n} \frac{\partial \ell_{t}(\widetilde{\theta})}{\partial \theta} \frac{\partial \ell_{t}(\widehat{\theta})}{\partial \theta^{\top}}=\sum_{t=1}^{n}\left(\frac{\partial \ell_{t}(\widetilde{\theta})}{\partial \beta_{1}}, \ldots, \frac{\partial \ell_{t}(\widetilde{\theta})}{\partial \beta_{k}} \frac{\partial \ell_{t}(\widetilde{\theta})}{\partial \gamma_{1}}, \ldots, \frac{\partial \ell_{t}(\widetilde{\theta})}{\partial \gamma_{m}}\right)\left(\begin{array}{c}
\frac{\partial \ell_{t}(\widehat{\theta})}{\partial \beta_{1}} \\
\cdots \\
\frac{\partial \ell_{t}(\widehat{\theta})}{\partial \beta_{k}} \\
\frac{\partial \ell_{t}(\widehat{\theta})}{\partial \gamma_{1}} \\
\cdots \\
\frac{\partial \ell_{t}(\widehat{\theta})}{\partial \gamma_{m}}
\end{array}\right)=\left[\begin{array}{cc}
\widehat{\Upsilon}_{\beta \beta} & \widehat{\Upsilon}_{\beta \gamma} \\
\widehat{\Upsilon}_{\gamma \beta} & \widehat{\Upsilon}_{\gamma \gamma}
\end{array}\right] \\
& \widehat{\Upsilon}_{\beta \beta ; i, j}=\sum_{t=1}^{n}\left(\frac{\partial \ell_{t}(\tilde{\theta})}{\partial \beta_{i}} \frac{\partial \ell_{t}(\widehat{\theta})}{\partial \beta_{j}}\right)=\sum_{t=1}^{n}\left(\frac{\partial \ell_{t}(\widetilde{\theta})}{\partial \mu_{t}} \frac{\partial \mu_{t}(\widetilde{\theta})}{\partial \eta_{t}} \frac{\partial \eta_{t}(\widetilde{\theta})}{\partial \beta_{1}}\right)\left(\frac{\partial \ell_{t}(\widehat{\theta})}{\partial \mu_{t}} \frac{\partial \mu_{t}(\widehat{\theta})}{\partial \eta_{t}} \frac{\partial \eta_{t}(\widehat{\theta})}{\partial \beta_{1}}\right) \\
& =\sum_{t=1}^{n}\left(\frac{1}{\widetilde{\sigma}_{t}}-\frac{1}{\widetilde{\sigma}_{t}} \exp \left(-\frac{y_{t}-\widetilde{\mu}_{t}}{\widetilde{\sigma}_{t}}\right)\right) \frac{\partial \mu_{t}(\tilde{\theta})}{\partial \eta_{t}} \frac{\partial \eta_{t}(\widetilde{\theta})}{\partial \beta_{i}}\left(\frac{1}{\widehat{\sigma}_{t}}-\frac{1}{\widehat{\sigma}_{t}} \exp \left(-\frac{y_{t}-\widehat{\mu}_{t}}{\widehat{\sigma}_{t}}\right)\right) \frac{\partial \mu_{t}(\widehat{\theta})}{\partial \eta_{t}} \frac{\partial \eta_{t}(\widehat{\theta})}{\partial \beta_{j}}
\end{aligned}
$$

então

$$
\widehat{\Upsilon}_{\beta \beta}=\widehat{X}^{\top} \widehat{\sigma}^{-1} \widehat{T}\left(\mathcal{I}-\widehat{\mathcal{Z}}^{\dagger}\right)\left(\mathcal{I}-\widetilde{\mathcal{Z}}^{\dagger}\right) \widetilde{\sigma}^{-1} \widetilde{T} \widetilde{X}
$$

De forma análoga podemos obter $\widehat{\Upsilon}_{\beta \gamma}, \widehat{\Upsilon}_{\gamma \beta}$ e $\widehat{\Upsilon}_{\gamma \gamma}$. 


\section{E.4 Fraser, Reid \& Wu (1999)}

Temos, por (2.4) e (2.5), que

$$
\begin{gathered}
\frac{\partial \ell(\theta)}{\partial y_{t}}=-\frac{1}{\sigma_{t}}+\frac{1}{\sigma_{t}} \exp \left(-\frac{y_{t}-\mu_{t}}{\sigma_{t}}\right)=\frac{1}{\sigma_{t}}\left(-1+\exp \left(-\frac{y_{t}-\mu_{t}}{\sigma_{t}}\right)\right), \\
\frac{\partial^{2} \ell(\theta)}{\partial y_{t} \partial \mu_{t}}=\frac{1}{\sigma_{t}^{2}} \exp \left(-\frac{y_{t}-\mu_{t}}{\sigma_{t}}\right)
\end{gathered}
$$

$\mathrm{e}$

$$
\frac{\partial^{2} \ell(\theta)}{\partial y_{t} \partial \sigma_{t}}=\frac{1}{\sigma_{t}^{2}}-\frac{1}{\sigma_{t}^{2}} \exp \left(-\frac{y_{t}-\mu_{t}}{\sigma_{t}}\right)+\frac{1}{\sigma_{t}} \exp \left(-\frac{y_{t}-\mu_{t}}{\sigma_{t}}\right)\left(\frac{y_{t}-\mu_{t}}{\sigma_{t}^{2}}\right)
$$

Então

$$
\begin{aligned}
\ell_{; y}(\theta) & =\frac{\partial \ell(\theta)}{\partial y}=\left(\frac{\partial \ell(\theta)}{\partial y_{1}}, \ldots, \frac{\partial \ell(\theta)}{\partial y_{n}}\right) \\
& =\left(\frac{1}{\sigma_{1}}\left(-1+\exp \left(-\frac{y_{1}-\mu_{1}}{\sigma_{1}}\right)\right), \ldots, \frac{1}{\sigma_{n}}\left(-1+\exp \left(-\frac{y_{n}-\mu_{n}}{\sigma_{n}}\right)\right)\right)=\iota \sigma^{-1}\left(-\mathcal{I}+\mathcal{Z}^{\dagger}\right),
\end{aligned}
$$

$\mathrm{e}$

$$
\ell_{\theta ; y}(\theta)=\frac{\partial \ell_{y}(\theta)}{\partial \theta^{\top}}=\left(\begin{array}{cccc}
\frac{\partial^{2} \ell(\theta)}{\partial y_{1} \partial \mu_{1}} \frac{\partial \mu_{1}}{\partial \eta_{1}} \frac{\partial \eta_{1}}{\partial \beta_{1}} & \ldots & \frac{\partial^{2} \ell(\theta)}{\partial y_{n} \partial \mu_{1}} \frac{\partial \mu_{1}}{\partial \eta_{1}} \frac{\partial \eta_{1}}{\partial \beta_{1}} \\
\ldots \ldots & \ldots & \ldots & \ldots \ldots \ldots \\
\frac{\partial^{2} \ell(\theta)}{\partial y_{1} \partial \mu_{1}} \frac{\partial \mu_{1}}{\partial \eta_{1}} \frac{\partial \eta_{1}}{\partial \beta_{k}} & \ldots & \frac{\partial^{2} \ell(\theta)}{\partial y_{n} \partial \sigma_{1}} \frac{\partial \mu_{1}}{\partial \eta_{1}} \frac{\partial \eta_{1}}{\partial \beta_{k}} \\
\frac{\partial^{2} \ell(\theta)}{\partial y_{1} \partial \sigma_{1}} \frac{\partial \sigma_{1}}{\partial \delta_{1}} \frac{\partial \delta_{1}}{\partial \gamma_{1}} & \ldots & \frac{\partial^{2} \ell(\theta)}{\partial y_{n} \partial \sigma_{1}} \frac{\partial \sigma_{1}}{\partial \delta_{1}} \frac{\partial \delta_{1}}{\partial \gamma_{1}} \\
\ldots \ldots \ldots & \ldots \ldots & \ldots & \ldots \ldots \ldots \ldots \\
\frac{\partial^{2} \ell(\theta)}{\partial y_{1} \partial \sigma_{1}} \frac{\partial \sigma_{1}}{\partial \delta_{1}} \frac{\partial \delta_{1}}{\partial \gamma_{m}} & \ldots & \frac{\partial^{2} \ell(\theta)}{\partial y_{n} \partial \sigma_{1}} \frac{\partial \sigma_{1}}{\partial \delta_{1}} \frac{\partial \delta_{1}}{\partial \gamma_{m}}
\end{array}\right)=\left(\begin{array}{c}
X^{\top} T \sigma^{-2} \mathcal{Z}^{\dagger} \\
Z^{\top} H \sigma^{-2}\left(\mathcal{I}-\mathcal{Z}^{\dagger}+\mathcal{Z}^{\dagger}\right)
\end{array}\right)
$$

que é uma matriz $(k+m) \times n$.

A função de distribuição acumulada é dada por

$$
F(y)=\exp \left(-\exp \left(-\frac{y_{t}-\mu_{t}}{\sigma_{t}}\right)\right)
$$

e a função densidade de probabilidade é dada por (2.1) logo

$$
\frac{\partial F\left(y_{t}, \theta\right)}{\partial \beta_{i}}=-\exp \left(-\exp \left(-\frac{y_{t}-\mu_{t}}{\sigma_{t}}\right)\right) \exp \left(-\frac{y_{t}-\mu_{t}}{\sigma_{t}}\right) \frac{1}{\sigma_{t}} \frac{\partial \mu_{t}}{\partial \eta_{t}} \frac{\partial \eta_{t}}{\beta_{i}}=-f\left(y_{t}, \theta\right) \frac{\partial \mu_{t}}{\partial \eta_{t}} \frac{\partial \eta_{t}}{\beta_{i}}
$$

$\mathrm{e}$

$$
\frac{\partial F\left(y_{t}, \theta\right)}{\partial \gamma_{i}}=-\exp \left(-\exp \left(-\frac{y_{t}-\mu_{t}}{\sigma_{t}}\right)\right) \exp \left(-\frac{y_{t}-\mu_{t}}{\sigma_{t}}\right)\left(\frac{y_{t}-\mu_{t}}{\sigma_{t}^{2}}\right) \frac{\partial \sigma_{t}}{\partial \delta_{t}} \frac{\partial \delta_{t}}{\gamma_{i}}
$$




$$
=-f\left(y_{t}, \theta\right)\left(\frac{y_{t}-\mu_{t}}{\sigma_{t}}\right) \frac{\partial \sigma_{t}}{\partial \delta_{t}} \frac{\partial \delta_{t}}{\gamma_{i}}
$$

portanto

$$
\widehat{V}=\left(-\frac{\frac{\partial F\left(y_{t}, \widehat{\theta}\right)}{\partial \theta_{i}}}{f\left(y_{t}, \widehat{\theta}\right)}\right)=\left(\begin{array}{ll}
\widehat{T} \widehat{X} & \widehat{H} \widehat{\mathcal{Z}} \widehat{Z}
\end{array}\right)
$$

é uma matriz $n \times(k+m)$. Por (E.15), (E.16) e (E.17) temos que

$$
\begin{aligned}
& \tilde{\ell}_{; \widehat{\theta}}(\widehat{\theta})-\widetilde{\ell}_{; \widehat{\theta}}(\widetilde{\theta})=\ell_{; y}(\widehat{\theta}) \widehat{V}\left(\ell_{\theta ; y}(\widehat{\theta}) \widehat{V}\right)^{-1} \widehat{J}-\ell_{; y}(\widetilde{\theta}) \widehat{V}\left(\ell_{\theta ; y}(\widehat{\theta}) \widehat{V}\right)^{-1} \widehat{J} \\
& =\left(\ell_{; y}(\widehat{\theta})-\ell_{; y}(\widetilde{\theta})\right) \widehat{V}\left(\ell_{\theta ; y}(\widehat{\theta}) \widehat{V}\right)^{-1} \widehat{J}=\iota\left(\widehat{\sigma}^{-1}\left(-\mathcal{I}+\widehat{\mathcal{Z}}^{\dagger}\right)-\widetilde{\sigma}^{-1}\left(-\mathcal{I}+\widetilde{\mathcal{Z}}^{\dagger}\right)\right)(\widehat{T} \widehat{X} \quad \widehat{H} \widehat{\mathcal{Z}} \widehat{Z}) \\
& \left(\left(\begin{array}{c}
\widehat{X}^{\top} \widehat{T} \widehat{\sigma}^{-2} \widehat{\mathcal{Z}}^{\dagger} \\
\widehat{Z}^{\top} \widehat{H} \widehat{\sigma}^{-2}\left(\mathcal{I}-\widehat{\mathcal{Z}}^{\dagger}+\widehat{\mathcal{Z}} \widehat{\mathcal{Z}}^{\dagger}\right)
\end{array}\right)\left(\begin{array}{ll}
\widehat{T} \widehat{X} & \widehat{H} \widehat{\mathcal{Z}} \widehat{Z})
\end{array}\right)\right)^{-1} \widehat{J}, \\
& \tilde{\ell}_{\theta ; \hat{\theta}}(\widetilde{\theta})=\ell_{\theta ; y}(\widetilde{\theta}) \widehat{V}\left(\ell_{\theta ; y}(\widehat{\theta}) \widehat{V}\right)^{-1} \widehat{J}=
\end{aligned}
$$

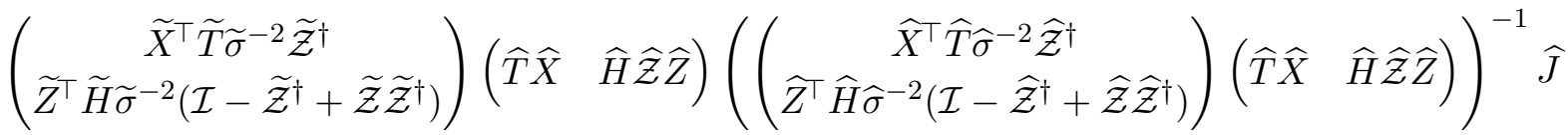

e $\widetilde{\ell}_{\psi ; \widehat{\theta}}(\widetilde{\theta})$ é obtida de $\widetilde{\ell}_{\theta ; \widetilde{\theta}}(\widetilde{\theta})$ excluindo-se a linha da matriz relativa ao parâmetro do teste. Temos

$$
\begin{aligned}
& \widetilde{U}=\frac{\left|\left(\begin{array}{c}
\tilde{\ell}_{; \widehat{\theta}}(\widehat{\theta})-\tilde{\ell}_{; \widehat{\theta}}(\widetilde{\theta}) \\
\tilde{\ell}_{\psi ; \widehat{\theta}}(\tilde{\theta})
\end{array}\right)\right|}{\left|\widetilde{J}_{\psi \psi}\right|^{1 / 2}|\widehat{J}|^{1 / 2}}= \\
& \left|\left(\begin{array}{c}
\iota\left(\widehat{\sigma}^{-1}\left(-\mathcal{I}+\widehat{\mathcal{Z}}^{\dagger}\right)-\widetilde{\sigma}^{-1}\left(-\mathcal{I}+\widetilde{\mathcal{Z}}^{\dagger}\right)\right) \\
\widetilde{A}_{\psi}
\end{array}\right)\left(\begin{array}{ll}
\widehat{T} \widehat{X} & \widehat{H} \widehat{\mathcal{Z}} \widehat{Z}
\end{array}\right)\left(\begin{array}{ll}
\widehat{A}(\widehat{T} \widehat{X} & \widehat{H} \widehat{\mathcal{Z}} \widehat{Z})
\end{array}\right)^{-1}\right|\left|\widetilde{J}_{\psi \psi}\right|^{-1 / 2}|\widehat{J}|^{1 / 2}
\end{aligned}
$$

em que $A=\left(\begin{array}{c}X^{\top} T \sigma^{-2} \mathcal{Z}^{\dagger} \\ Z^{\top} H \sigma^{-2}\left(\mathcal{I}-\mathcal{Z}^{\dagger}+\mathcal{Z} \mathcal{Z}^{\dagger}\right)\end{array}\right)$. 


\section{Apêndice F}

\section{Generalizações da distribuição valor extremo máximo ou Gum- bel}

\section{F.1 Distribuição valor extremo generalizada}

Seja $X$ uma variável aleatória com distribuição $\operatorname{GEV}(\mu, \sigma, \alpha)$. A função densidade da distribuição GEV, derivada da função de distribuição acumulada (1.2), é

$$
f_{G E V}(x)=\exp \left\{-\left[1+\alpha\left(\frac{x-\mu}{\sigma}\right)\right]^{-1 / \alpha}\right\}\left[1+\alpha\left(\frac{x-\mu}{\sigma}\right)\right]^{-1 / \alpha-1}\left(\frac{1}{\sigma}\right)
$$

definida em $\{x: 1+\alpha(x-\mu) / \sigma>0\}$, em que $-\infty<\mu<\infty, \sigma>0$ e $-\infty<\alpha<\infty$. Se $\alpha>0$ então $x>(\mu-\sigma / \alpha)$ e a esperança de $X$ é

$$
\begin{aligned}
\mathrm{E}(X) & =\int_{(\mu-\sigma / \alpha)}^{\infty} x \exp \left\{-\left[1+\alpha\left(\frac{x-\mu}{\sigma}\right)\right]^{-1 / \alpha}\right\}\left[1+\alpha\left(\frac{x-\mu}{\sigma}\right)\right]^{-1 / \alpha-1}\left(\frac{1}{\sigma}\right) d x \\
& =\int_{0}^{\infty}\left(\mu-\frac{\sigma}{\alpha}+\frac{\sigma y^{-\alpha}}{\alpha}\right) \exp \{-y\} d y=\left(\mu-\frac{\sigma}{\alpha}\right)+\frac{\sigma}{\alpha} \Gamma(1-\alpha),
\end{aligned}
$$

considerando $y=\left[1+\alpha\left(\frac{x-\mu}{\sigma}\right)\right]^{-1 / \alpha}$ e $\int_{0}^{\infty} x^{\nu-1} \exp (-\mu x) d x=1 / \mu^{\nu} \Gamma(\nu)$ se $\operatorname{Re} \mu>0$ e $\operatorname{Re} \nu>0$ (Gradshteyn \& Ryzhik, 2000, equação 3.381.4). Analogamente, se $\alpha<0$ a esperança é a mesma. Se $\alpha=0$

$$
\mathrm{E}(X)=\lim _{\alpha \rightarrow 0}\left(\mu-\frac{\sigma}{\alpha}(1-\Gamma(1-\alpha))\right)=\mu-\sigma \lim _{\alpha \rightarrow 0} \frac{1-\Gamma(1-\alpha)}{\alpha}=\mu-\sigma \Gamma^{\prime}(1)=\mu+\sigma \mathcal{E},
$$

em que $\mathcal{E}=-\Gamma^{\prime}(1)$ é a constante de Euler; $\mathcal{E} \approx 0,5772$. Usando as mesmas substituições acima, obtemos

$$
\begin{aligned}
& E\left((X-E(X))^{n}\right) \\
= & \int_{(\mu-\sigma / \alpha)}^{\infty}(x-E(X))^{n} \exp \left\{-\left[1+\alpha\left(\frac{x-\mu}{\sigma}\right)\right]^{-1 / \alpha}\right\}\left[1+\alpha\left(\frac{x-\mu}{\sigma}\right)\right]^{-1 / \alpha-1}\left(\frac{1}{\sigma}\right) d x \\
= & \int_{\infty}^{0}\left(\mu+\frac{\sigma\left(y^{-\alpha}-1\right)}{\alpha}-\left(\left(\mu-\frac{\sigma}{\alpha}\right)+\frac{\sigma}{\alpha} \Gamma(1-\alpha)\right)\right)^{n} \exp \{-y\}(-d y) \\
= & \int_{0}^{\infty}\left(\frac{\sigma}{\alpha}\left(y^{-\alpha}-\Gamma(1-\alpha)\right)\right)^{n} \exp \{-y\} d y,
\end{aligned}
$$




$$
\begin{gathered}
E\left((X-E(X))^{2}\right)=\int_{0}^{\infty}\left(\frac{\sigma}{\alpha}\left(y^{-\alpha}-\Gamma(1-\alpha)\right)\right)^{2} \exp \{-y\} d y \\
=\left(\frac{\sigma}{\alpha}\right)^{2} \int_{0}^{\infty}\left(y^{-2 \alpha}-2 \Gamma(1-\alpha) y^{-\alpha}+\Gamma^{2}(1-\alpha)\right) \exp \{-y\} d y=\left(\frac{\sigma}{\alpha}\right)^{2}\left(\Gamma(1-2 \alpha)-\Gamma^{2}(1-\alpha)\right) \\
E\left((X-E(X))^{3}\right)=\left(\frac{\sigma}{\alpha}\right)^{3}\left(\Gamma(1-3 \alpha)-3 \Gamma(1-2 \alpha) \Gamma(1-\alpha)+2 \Gamma^{3}(1-\alpha)\right) \\
E\left((X-E(X))^{4}\right)=\left(\frac{\sigma}{\alpha}\right)^{4}\left(\Gamma(1-4 \alpha)-4 \Gamma(1-3 \alpha) \Gamma(1-\alpha)+6 \Gamma(1-2 \alpha) \Gamma^{2}(1-\alpha)-3 \Gamma^{4}(1-\alpha)\right) .
\end{gathered}
$$

Os coeficientes de assimetria e de curtose são dados, respectivamente, por

$$
\gamma_{1, G E V}=\frac{E\left((X-E(X))^{3}\right)}{\left(E\left((X-E(X))^{2}\right)\right)^{3 / 2}}=\frac{\left(\Gamma(1-3 \alpha)-3 \Gamma(1-2 \alpha) \Gamma(1-\alpha)+2 \Gamma^{3}(1-\alpha)\right)}{\left(\left(\Gamma(1-2 \alpha)-\Gamma^{2}(1-\alpha)\right)\right)^{3 / 2}}
$$

e

$$
\gamma_{2, G E V}=\frac{E\left((X-E(X))^{4}\right)}{\left(E\left((X-E(X))^{2}\right)\right)^{2}}=\frac{\left(\Gamma(1-4 \alpha)-4 \Gamma(1-3 \alpha) \Gamma(1-\alpha)+6 \Gamma(1-2 \alpha) \Gamma^{2}(1-\alpha)-3 \Gamma^{4}(1-\alpha)\right)}{\left(\left(\Gamma(1-2 \alpha)-\Gamma^{2}(1-\alpha)\right)\right)^{2}} .
$$

Analogamente obtém-se, para $\alpha<0$,

$$
\gamma_{1, G E V}=-\frac{\left(\Gamma(1-3 \alpha)-3 \Gamma(1-2 \alpha) \Gamma(1-\alpha)+2 \Gamma^{3}(1-\alpha)\right)}{\left(\left(\Gamma(1-2 \alpha)-\Gamma^{2}(1-\alpha)\right)\right)^{3 / 2}}
$$

e $\gamma_{2}$ como anteriormente.

Se $\alpha=0$

$$
\begin{aligned}
& \operatorname{var}(X)=\lim _{\alpha \rightarrow 0}\left(\frac{\sigma}{\alpha}\right)^{2}\left(\Gamma(1-2 \alpha)-\Gamma^{2}(1-\alpha)\right)=\sigma^{2} \lim _{\alpha \rightarrow 0}\left(\frac{\Gamma(1-2 \alpha)-\Gamma^{2}(1-\alpha)}{\alpha^{2}}\right) \\
= & \sigma^{2} \lim _{\alpha \rightarrow 0}\left(\frac{2 \Gamma^{\prime}(1-2 \alpha)-2 \Gamma(1-\alpha) \Gamma^{\prime}(1-\alpha)}{2 \alpha}\right) \\
= & \sigma^{2} \lim _{\alpha \rightarrow 0}\left(\frac{4 \Gamma^{\prime \prime}(1-2 \alpha)-2\left(\left(\Gamma^{\prime}(1-\alpha)\right)^{2}+\Gamma(1-\alpha) \Gamma^{\prime \prime}(1-\alpha)\right)}{2}\right) \\
= & \left.\sigma^{2}\left(2 \Gamma^{\prime \prime}(1)-\left(\left(\Gamma^{\prime}(1)\right)^{2}+\Gamma^{\prime \prime}(1)\right)\right)=\sigma^{2}\left(\Gamma^{\prime \prime}(1)-\Gamma^{\prime}(1)\right)^{2}\right)=\sigma^{2} \psi^{\prime}(1)=\sigma^{2} \pi^{2} / 6,
\end{aligned}
$$

em que $\psi(x)=d \ln \Gamma(x) / d x$ é a função psi, $\psi^{\prime}(1)=\pi^{2} / 6$ (Gradshteyn \& Ryzhik, 2000, equação 8.366.8) e a terceira e quarta igualdades são obtidas usando a regra de L'Hopital.

Se $\alpha>0$ então $x>(\mu-\sigma / \alpha)$ e a mediana $m$ de $X$ é dada por

$$
\frac{1}{2}=\int_{(\mu-\sigma / \alpha)}^{m} \exp \left\{-\left[1+\alpha\left(\frac{x-\mu}{\sigma}\right)\right]^{-1 / \alpha}\right\}\left[1+\alpha\left(\frac{x-\mu}{\sigma}\right)\right]^{-1 / \alpha-1}\left(\frac{1}{\sigma}\right) d x
$$




$$
=\int_{\infty}^{\left[1+\alpha\left(\frac{m-\mu}{\sigma}\right)\right]^{-1 / \alpha}} \exp \{-y\}(-d y)=\exp \left\{-\left[1+\alpha\left(\frac{m-\mu}{\sigma}\right)\right]^{-1 / \alpha}\right\},
$$

considerando a tansformação $y=\left[1+\alpha\left(\frac{x-\mu}{\sigma}\right)\right]^{-1 / \alpha}$. Portanto,

$$
m=\mu+\sigma\left((\ln 2)^{-\alpha}-1\right) / \alpha .
$$

Analogamente, se $\alpha<0$ a mediana é a mesma. Se $\alpha=0$

$$
m=\lim _{\alpha \rightarrow 0}\left(\mu+\sigma\left(\ln (2)^{-\alpha}-1\right) / \alpha\right)=\mu-\sigma \ln \ln 2,
$$

pois, $\ln x=\lim _{\epsilon \rightarrow 0}\left(\left(x^{\epsilon}-1\right) / \epsilon\right)$ (Gradshteyn \& Ryzhik, 2000, equação 1.512.4).

Quando $\alpha=0$, a distribuição $G E V(\mu, \sigma, \alpha)$ é a distribuição valor extremo máximo ou Gumbel e $X \sim V E_{\max }(\mu, \sigma)=\operatorname{Gumbel}(\mu, \sigma) \operatorname{com}$ função densidade de probabilidade (2.1), então

$$
\begin{aligned}
E\left((X-E(X))^{n}\right) & =\int_{-\infty}^{\infty}(x-(\mu+\mathcal{E} \sigma))^{n} \frac{1}{\sigma} \exp \left(-\exp \left(-\frac{x-\mu}{\sigma}\right)\right) \exp \left(-\frac{x-\mu}{\sigma}\right) d x \\
& =\int_{0}^{\infty}(-\sigma)^{n}(\ln y+\mathcal{E})^{n} \exp (-y) d y
\end{aligned}
$$

usando a transformação $y=\exp (-(x-\mu) / \sigma)$. Temos que a função gama é definida por $\Gamma(z)=$ $\int_{0}^{\infty} t^{z-1} \exp (-t) d t$, sua $n$-ésima derivada $\Gamma^{(n)}(1)=\int_{0}^{\infty}(\ln t)^{n} \exp (-t) d t \mathrm{e}(a+b)^{n}=\sum_{i=0}^{n}\left(\begin{array}{l}n \\ i\end{array}\right) a^{(n-i)} b^{i}$ então obtemos

$$
E\left((X-E(X))^{3}\right)=-\sigma^{3}\left(\Gamma^{(3)}(1)+3 \mathcal{E} \Gamma^{(2)}(1)+3 \mathcal{E}^{2} \Gamma^{(1)}(1)+\mathcal{E}^{3}\right)
$$

$\mathrm{e}$

$$
E\left((X-E(X))^{4}\right)=\sigma^{4}\left(\Gamma^{(4)}(1)+4 \mathcal{E} \Gamma^{(3)}(1)+6 \mathcal{E}^{2} \Gamma^{(2)}(1)+4 \mathcal{E}^{3} \Gamma^{(1)}(1)+\mathcal{E}^{4}\right) .
$$

Os coeficientes de assimetria e de curtose podem ser obtidos, com o software Mathematica (Wolfram Research, 2012), como segue

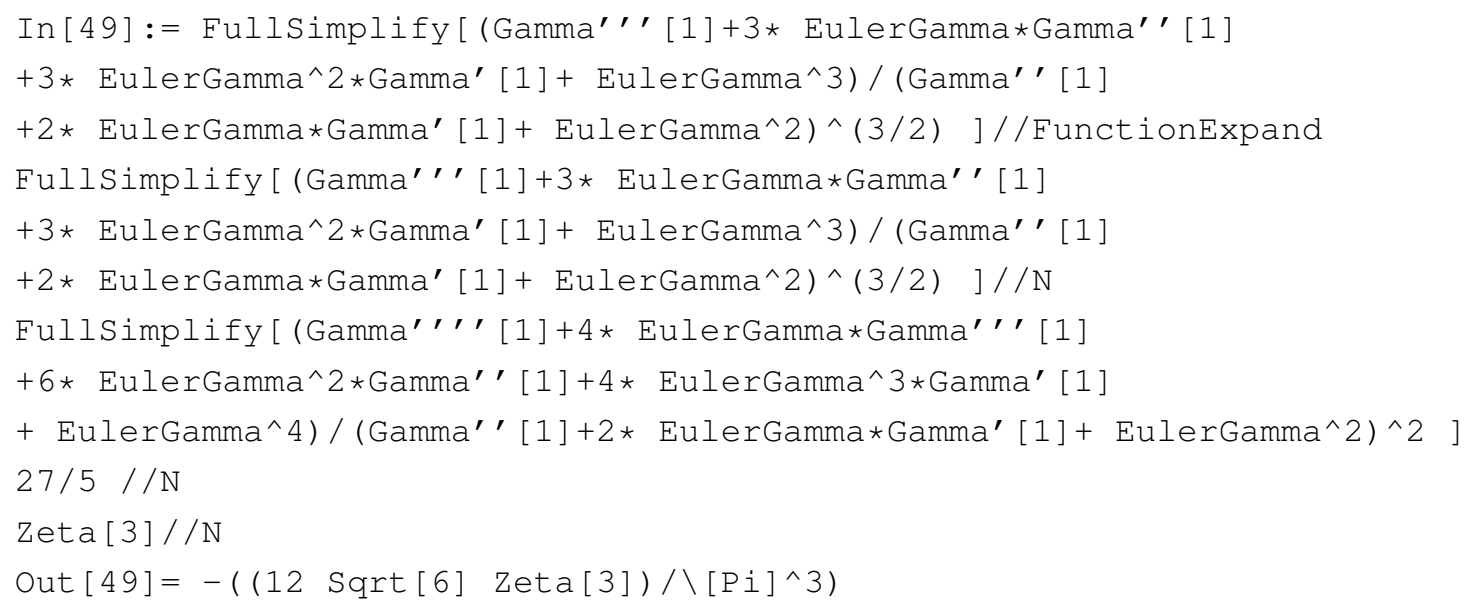


Out $[50]=-1.13955$

Out $[51]=27 / 5$

Out $[52]=5.4$

Out $[53]=1.20206$

Segue que a assimetria e a curtose da Gumbel não dependem de parâmetros e são constantes iguais a $(12 \sqrt{(6)} \zeta(3)) / \pi^{3} \approx 1,139547$ e 5,4, respectivamente, em que $\zeta(s)=\sum_{k=1}^{\infty} k^{-s}$, Re $s>1$ é a função zeta de Riemann e $\zeta(3) \approx 1,2$.

\section{F.2 Distribuição Gumbel exponencializada}

Podemos obter os coeficientes de assimetria e de curtose da distribuição $E G u(\mu, \sigma, \alpha)$ com a seguinte rotina no software Mathematica (Wolfram Research, 2012)

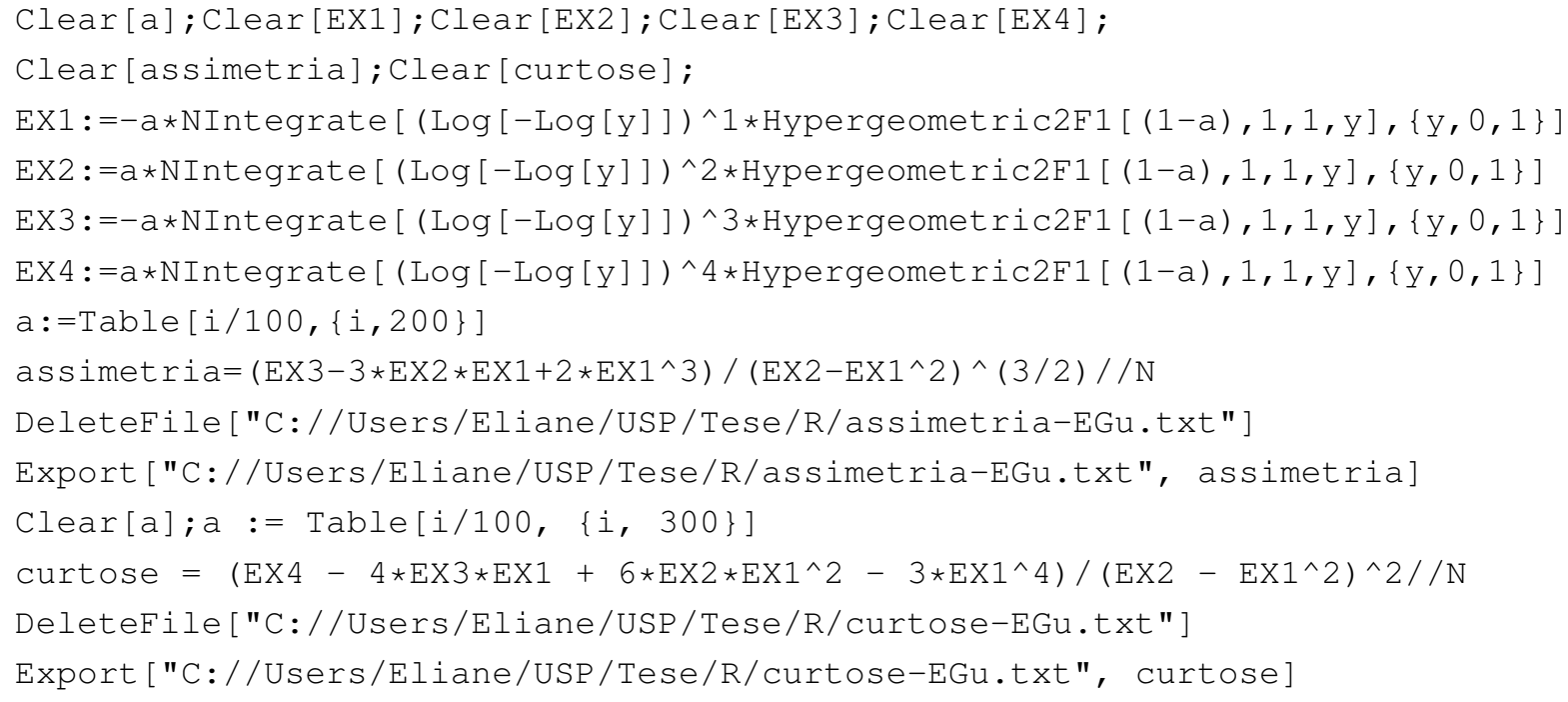

\section{F.3 Distribuição valor extremo transmutada}

O quantil $x_{p}$ é tal que $F\left(x_{p}\right)=p$, logo

$$
p=(1+\alpha) \exp \left[-\exp \left(-\frac{x_{p}-\mu}{\sigma}\right)\right]-\alpha \exp \left[-2 \exp \left(-\frac{x_{p}-\mu}{\sigma}\right)\right]
$$

ou

$$
\alpha\left(\exp \left[-\exp \left(-\frac{x_{p}-\mu}{\sigma}\right)\right]\right)^{2}-(1+\alpha) \exp \left[-\exp \left(-\frac{x_{p}-\mu}{\sigma}\right)\right]+p=0
$$

$\log 0$

$$
\exp \left[-\exp \left(-\frac{x_{p}-\mu}{\sigma}\right)\right]=\frac{1+\alpha \pm \sqrt{(1+\alpha)^{2}-4 \alpha p}}{2 \alpha}
$$

$\mathrm{e}$

$$
x_{p}=\mu-\sigma \ln \left(-\ln \left(\frac{1+\alpha-\sqrt{(1+\alpha)^{2}-4 \alpha p}}{2 \alpha}\right)\right) .
$$

Conforme Aryal \& Tsokos (2009, p. 1404) temos que

$$
E\left(X^{n}\right)=\left.\sum_{k=0}^{n}(-1)^{k}\left(\begin{array}{l}
n \\
k
\end{array}\right) \sigma^{k} \mu^{n-k}\left[(1+\alpha) \frac{\partial^{k}}{\partial \nu^{k}} \Gamma(\nu)-2 \alpha \frac{\partial^{k}}{\partial \nu^{k}}\left[2^{-\nu} \Gamma(\nu)\right]\right]\right|_{\nu=1}
$$




$$
\begin{aligned}
& \frac{\partial}{\partial \nu}\left[2^{-\nu} \Gamma(\nu)\right]=\frac{\partial 2^{-\nu}}{\partial \nu} \Gamma(\nu)+2^{-\nu} \Gamma^{\prime}(\nu)=2^{-\nu}\left(-\ln 2 \Gamma(\nu)+\Gamma^{\prime}(\nu)\right), \\
& \frac{\partial^{2}}{\partial \nu^{2}}\left[2^{-\nu} \Gamma(\nu)\right]=2^{-\nu}\left(\ln ^{2} 2 \Gamma(\nu)-2 \ln 2 \Gamma^{\prime}(\nu)+\Gamma^{(2)}(\nu)\right) \\
& \frac{\partial^{3}}{\partial \nu^{3}}\left[2^{-\nu} \Gamma(\nu)\right]=2^{-\nu}\left(-\ln ^{3} 2 \Gamma(\nu)+3 \ln ^{2} 2 \Gamma^{\prime}(\nu)-3 \ln 2 \Gamma^{(2)}(\nu)+\Gamma^{(3)}(\nu)\right) \\
& \frac{\partial^{4}}{\partial \nu^{4}}\left[2^{-\nu} \Gamma(\nu)\right]=2^{-\nu}\left(\ln ^{4} 2 \Gamma(\nu)-4 \ln ^{3} 2 \Gamma^{\prime}(\nu)+6 \ln 2 \Gamma^{(2)}(\nu)-4 \ln 2 \Gamma^{(3)}(\nu)+\Gamma^{(4)}(\nu)\right) \\
& \left.\frac{\partial}{\partial \nu}\left[2^{-\nu} \Gamma(\nu)\right]\right|_{\nu=1}=1 / 2\left(-\ln 2+\Gamma^{\prime}(1)\right) \\
& \left.\frac{\partial^{2}}{\partial \nu^{2}}\left[2^{-\nu} \Gamma(\nu)\right]\right|_{\nu=1}=1 / 2\left(\ln ^{2} 2-2 \ln 2 \Gamma^{\prime}(1)+\Gamma^{(2)}(1)\right) \\
& \left.\frac{\partial^{3}}{\partial \nu^{3}}\left[2^{-\nu} \Gamma(\nu)\right]\right|_{\nu=1}=1 / 2\left(-\ln ^{3} 2+3 \ln ^{2} 2 \Gamma^{\prime}(1)-3 \ln 2 \Gamma^{(2)}(1)+\Gamma^{(3)}(1)\right) \\
& \left.\frac{\partial^{4}}{\partial \nu^{4}}\left[2^{-\nu} \Gamma(\nu)\right]\right|_{\nu=1}=1 / 2\left(\ln ^{4} 2-4 \ln ^{3} 2 \Gamma^{\prime}(1)+6 \ln 2 \Gamma^{(2)}(1)-4 \ln 2 \Gamma^{(3)}(1)+\Gamma^{(4)}(1)\right) \\
& E(X)=\left.\sum_{k=0}^{1}(-1)^{k}\left(\begin{array}{l}
1 \\
k
\end{array}\right) \sigma^{k} \mu^{1-k}\left[(1+\alpha) \frac{\partial^{k}}{\partial \nu^{k}} \Gamma(\nu)-2 \alpha \frac{\partial^{k}}{\partial \nu^{k}}\left[2^{-\nu} \Gamma(\nu)\right]\right]\right|_{\nu=1} \\
& =\mu[(1+\alpha) \Gamma(1)-2 \alpha[1 / 2 \Gamma(1)]]-\sigma\left[(1+\alpha) \Gamma^{\prime}(1)-2 \alpha 1 / 2\left(-\ln 2+\Gamma^{\prime}(1)\right)\right] \\
& =\mu-\sigma \Gamma^{\prime}(1)-\alpha(\sigma \ln 2) \\
& =\mu+\sigma \mathcal{E}-\alpha \sigma \ln 2 \\
& E\left(X^{2}\right)=\left.\sum_{k=0}^{2}(-1)^{k}\left(\begin{array}{l}
2 \\
k
\end{array}\right) \sigma^{k} \mu^{2-k}\left[(1+\alpha) \frac{\partial^{k}}{\partial \nu^{k}} \Gamma(\nu)-2 \alpha \frac{\partial^{k}}{\partial \nu^{k}}\left[2^{-\nu} \Gamma(\nu)\right]\right]\right|_{\nu=1} \\
& =\mu^{2}[(1+\alpha) \Gamma(1)-2 \alpha[1 / 2 \Gamma(1)]]-2 \mu \sigma\left[(1+\alpha) \Gamma^{\prime}(1)-2 \alpha 1 / 2\left(-\ln 2+\Gamma^{\prime}(1)\right)\right] \\
& +\sigma^{2}\left[(1+\alpha) \Gamma^{(2)}(1)-2 \alpha 1 / 2\left(\ln ^{2} 2-2 \ln 2 \Gamma^{\prime}(1)+\Gamma^{(2)}(1)\right)\right] \\
& =\mu^{2}-2 \mu \sigma\left[\Gamma^{\prime}(1)+\alpha \ln 2\right]+\sigma^{2}\left[\Gamma^{(2)}(1)-\alpha\left(\ln ^{2} 2-2 \ln 2 \Gamma^{\prime}(1)\right]\right. \\
& =\mu^{2}-2 \mu \sigma \Gamma^{\prime}(1)+\sigma^{2} \Gamma^{(2)}(1)-\alpha\left(2 \mu \sigma \ln 2+\sigma^{2}\left(\ln ^{2} 2-2 \ln 2 \Gamma^{\prime}(1)\right)\right) \\
& \begin{aligned}
E\left(X^{3}\right)= & \left.\sum_{k=0}^{3}(-1)^{k}\left(\begin{array}{l}
3 \\
k
\end{array}\right) \sigma^{k} \mu^{3-k}\left[(1+\alpha) \frac{\partial^{k}}{\partial \nu^{k}} \Gamma(\nu)-2 \alpha \frac{\partial^{k}}{\partial \nu^{k}}\left[2^{-\nu} \Gamma(\nu)\right]\right]\right|_{\nu=1} \\
= & \mu^{3}[(1+\alpha) \Gamma(1)-2 \alpha[1 / 2 \Gamma(1)]]-3 \mu^{2} \sigma\left[(1+\alpha) \Gamma^{\prime}(1)-2 \alpha 1 / 2\left(-\ln 2+\Gamma^{\prime}(1)\right)\right] \\
& +3 \mu \sigma^{2}\left[(1+\alpha) \Gamma^{(2)}(1)-2 \alpha 1 / 2\left(\ln ^{2} 2-2 \ln 2 \Gamma^{\prime}(1)+\Gamma^{(2)}(1)\right)\right] \\
& -\sigma^{3}\left[(1+\alpha) \Gamma^{(3)}(1)-2 \alpha 1 / 2\left(-\ln ^{3} 2+3 \ln ^{2} 2 \Gamma^{\prime}(1)-3 \ln 2 \Gamma^{(2)}(1)+\Gamma^{(3)}(1)\right)\right] \\
= & \mu^{3}-3 \mu^{2} \sigma\left[\Gamma^{\prime}(1)+\alpha \ln 2\right]+3 \mu \sigma^{2}\left[\Gamma^{(2)}(1)-\alpha\left(\ln ^{2} 2-2 \ln 2 \Gamma^{\prime}(1)\right)\right] \\
& -\sigma^{3}\left[\Gamma^{(3)}(1)-\alpha\left(-\ln ^{3} 2+3 \ln ^{2} 2 \Gamma^{\prime}(1)-3 \ln 2 \Gamma^{(2)}(1)\right)\right]
\end{aligned}
\end{aligned}
$$




$$
\begin{aligned}
= & \mu^{3}-3 \mu^{2} \sigma \Gamma^{\prime}(1)-3 \mu^{2} \sigma \alpha \ln 2+3 \mu \sigma^{2} \Gamma^{(2)}(1)-3 \mu \sigma^{2} \alpha\left(\ln ^{2} 2-2 \ln 2 \Gamma^{\prime}(1)\right) \\
& -\sigma^{3} \Gamma^{(3)}(1)+\sigma^{3} \alpha\left(-\ln ^{3} 2+3 \ln ^{2} 2 \Gamma^{\prime}(1)-3 \ln 2 \Gamma^{(2)}(1)\right) \\
= & \mu^{3}-3 \mu^{2} \sigma \Gamma^{\prime}(1)+3 \mu \sigma^{2} \Gamma^{(2)}(1)-\sigma^{3} \Gamma^{(3)}(1)-\alpha\left(3 \mu^{2} \sigma \ln 2+3 \mu \sigma^{2}\left(\ln ^{2} 2-2 \ln 2 \Gamma^{\prime}(1)\right)\right. \\
& \left.-\sigma^{3}\left(-\ln ^{3} 2+3 \ln ^{2} 2 \Gamma^{\prime}(1)-3 \ln 2 \Gamma^{(2)}(1)\right)\right) \\
E\left(X^{4}\right)= & \left.\sum_{k=}^{4}(-1)^{k}\left(\begin{array}{l}
4 \\
k
\end{array}\right) \sigma^{k} \mu^{4-k}\left[(1+\alpha) \frac{\partial^{k}}{\partial \nu^{k}} \Gamma(\nu)-2 \alpha \frac{\partial^{k}}{\partial \nu^{k}}\left[2^{-\nu} \Gamma(\nu)\right]\right]\right|_{\nu=1} \\
= & \mu^{4}[(1+\alpha) \Gamma(1)-2 \alpha[1 / 2 \Gamma(1)]]-4 \mu^{3} \sigma\left[(1+\alpha) \Gamma^{\prime}(1)-2 \alpha 1 / 2\left(-\ln 2+\Gamma^{\prime}(1)\right)\right] \\
& +6 \mu^{2} \sigma^{2}\left[(1+\alpha) \Gamma^{(2)}(1)-2 \alpha 1 / 2\left(\ln ^{2} 2-2 \ln 2 \Gamma^{\prime}(1)+\Gamma^{(2)}(1)\right)\right] \\
& \left.-4 \mu \sigma^{3}\left[(1+\alpha) \Gamma^{(3)}(1)-2 \alpha 1 / 2\left(-\ln ^{3} 2+3 \ln 22 \Gamma^{\prime}(1)-3 \ln 2 \Gamma^{(2)}(1)+\Gamma^{(3)}(1)\right)\right)\right] \\
& \sigma^{4}\left[(1+\alpha) \Gamma^{(4)}(1)-2 \alpha 1 / 2\left(\left(\ln ^{4} 2-4 \ln 32 \Gamma^{\prime}(1)+6 \ln 2 \Gamma^{(2)}(1)-4 \ln 2 \Gamma^{(3)}(1)+\Gamma^{(4)}(1)\right)\right)\right] \\
= & \mu^{4}-4 \mu^{3} \sigma\left[\Gamma^{\prime}(1)+\alpha \ln ^{2} 2\right] \\
& +6 \mu^{2} \sigma^{2}\left[\Gamma^{(2)}(1)-\alpha\left(\ln ^{2} 2-2 \ln 2 \Gamma^{\prime}(1)\right)\right] \\
& -4 \mu \sigma^{3}\left[\Gamma^{(3)}(1)-\alpha\left(-\ln ^{3} 2+3 \ln 2 \Gamma^{\prime}(1)-3 \ln 2 \Gamma^{(2)}(1)\right)\right] \\
& \sigma^{4}\left[\Gamma^{(4)}(1)-\alpha\left(\left(\ln ^{4} 2-4 \ln ^{3} 2 \Gamma^{\prime}(1)+6 \ln 2 \Gamma^{(2)}(1)-4 \ln 2 \Gamma^{(3)}(1)\right)\right)\right] \\
= & \mu^{4}-4 \mu^{3} \sigma \Gamma^{\prime}(1)+6 \mu^{2} \sigma^{2} \Gamma^{(2)}(1)-4 \mu \sigma^{3} \Gamma^{(3)}(1)+\sigma^{4} \Gamma^{(4)}(1) \\
& -\alpha\left[4 \mu^{3} \sigma \ln ^{2} 2+6 \mu^{2} \sigma^{2}\left(\ln ^{2} 2-2 \ln 2 \Gamma^{\prime}(1)\right)\right. \\
& -4 \mu \sigma^{3}\left(-\ln 32+3 \ln ^{2} 2 \Gamma^{\prime}(1)-3 \ln 2 \Gamma^{(2)}(1)\right) \\
& \left.+\sigma^{4}\left(\left(\ln ^{4} 2-4 \ln ^{3} 2 \Gamma^{\prime}(1)+6 \ln 2 \Gamma^{(2)}(1)-4 \ln 2 \Gamma^{(3)}(1)\right)\right)\right] .
\end{aligned}
$$

Obtemos o coeficiente de assimetria e o excesso do coeficiente de curtose da distribuição $T E V(\mu, \sigma, \alpha)$ com o software Mathematica (Wolfram Research, 2012):

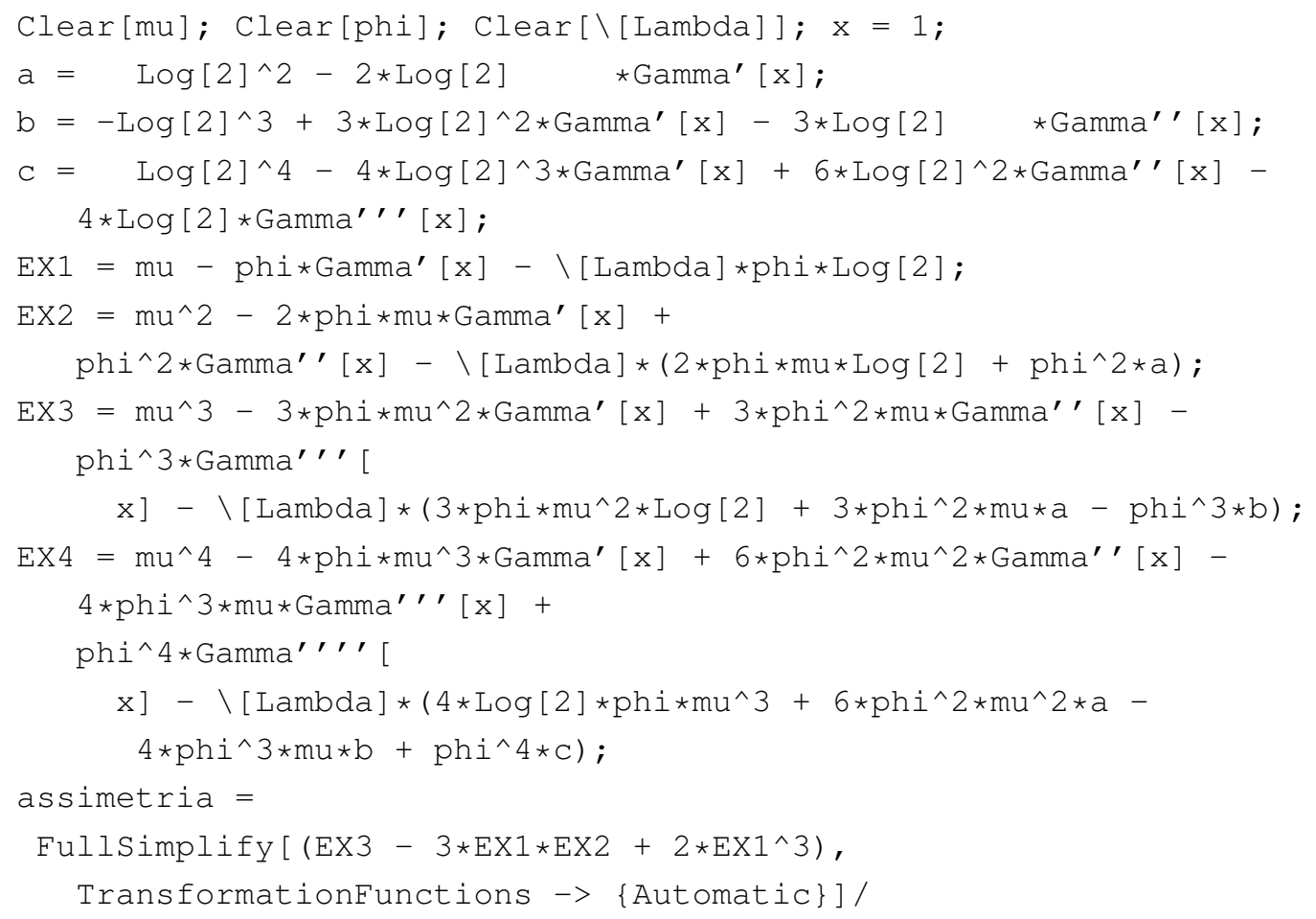




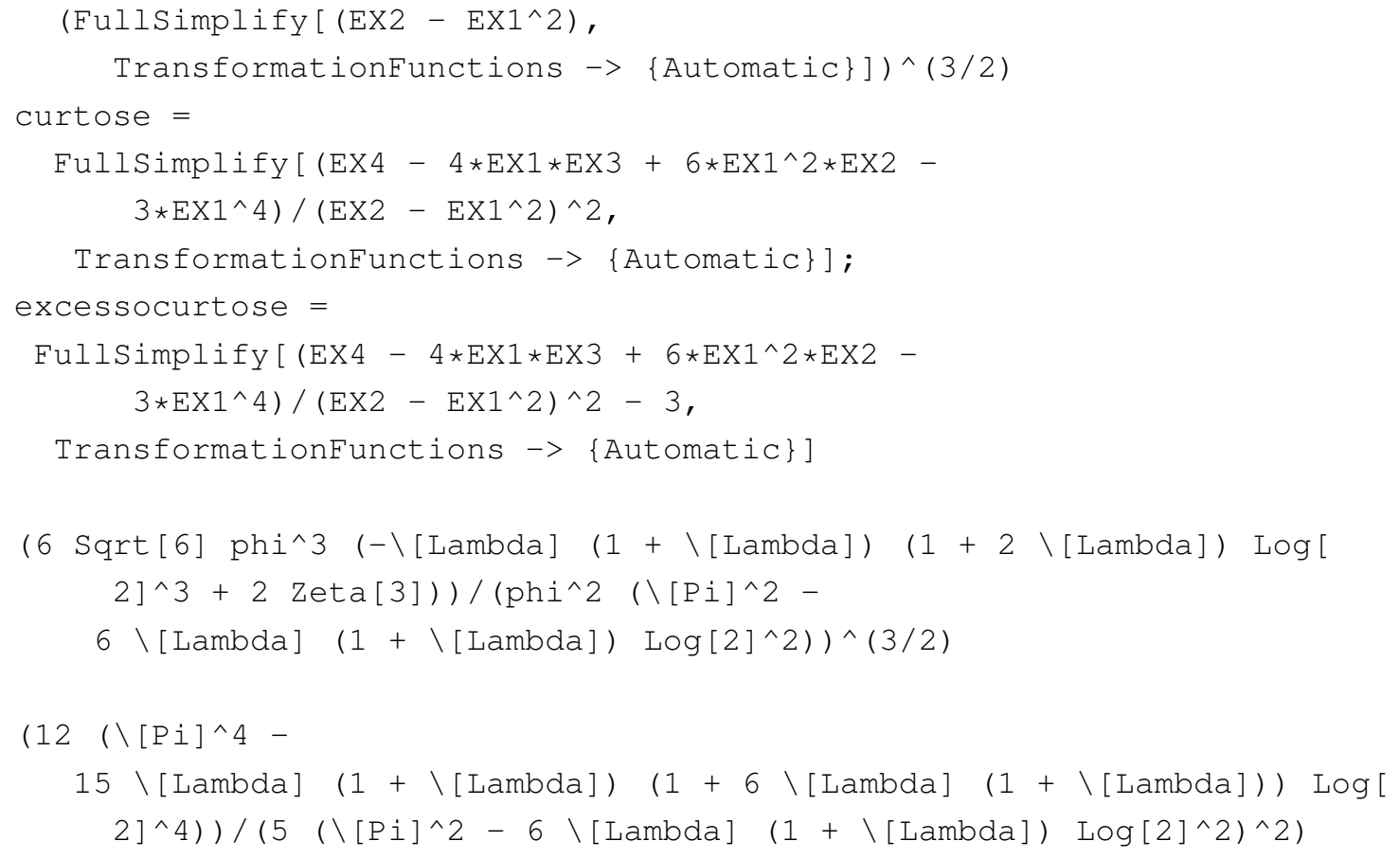

Então

$$
\begin{aligned}
\gamma_{1, T E V} & =\frac{6 \sqrt{(6) \sigma^{3}\left(2 \zeta(3)-(\ln 2)^{3} \alpha(1+\alpha)(1+2 \alpha)\right)}}{\left(\sigma^{2}\left(\pi^{2}-6(\ln 2)^{2} \alpha(1+\alpha)\right)\right)^{3 / 2}} \\
& =\frac{12 \sqrt{(6) \zeta(3)}}{\pi^{3}}\left(\frac{1-\left((\ln 2)^{3} / 2 \zeta(3)\right) \alpha(1+\alpha)(1+2 \alpha)}{\left(1-6(\ln 2 / \pi)^{2} \alpha(1+\alpha)\right)^{3 / 2}}\right) \\
& =\gamma_{1, E V} \frac{1-\left((\ln 2)^{3} / 2 \zeta(3)\right) \alpha(1+\alpha)(1+2 \alpha)}{\left(1-6(\ln 2 / \pi)^{2} \alpha(1+\alpha)\right)^{3 / 2}}
\end{aligned}
$$

$\mathrm{e}$

$$
\begin{aligned}
\gamma_{2, T E V}-3 & =\frac{12}{5}\left(\frac{\pi^{4}-15(\ln 2)^{4} \alpha(1+\alpha)(1+6 \alpha(1+\alpha))}{\left(\pi^{2}-6(\ln 2)^{2} \alpha(1+\alpha)\right)^{2}}\right) \\
& =\left(\gamma_{2, E V}-3\right) \frac{1-15(\ln 2 / \pi)^{4} \alpha(1+\alpha)(1+6 \alpha(1+\alpha))}{\left(1-6(\ln 2 / \pi)^{2} \alpha(1+\alpha)\right)^{2}}
\end{aligned}
$$

Os mínimos e máximos locais dos coeficientes de assimetria e de curtose para $-1 \leq \alpha \leq 1$ foram obtidos com o software Mathematica (Wolfram Research, 2012):

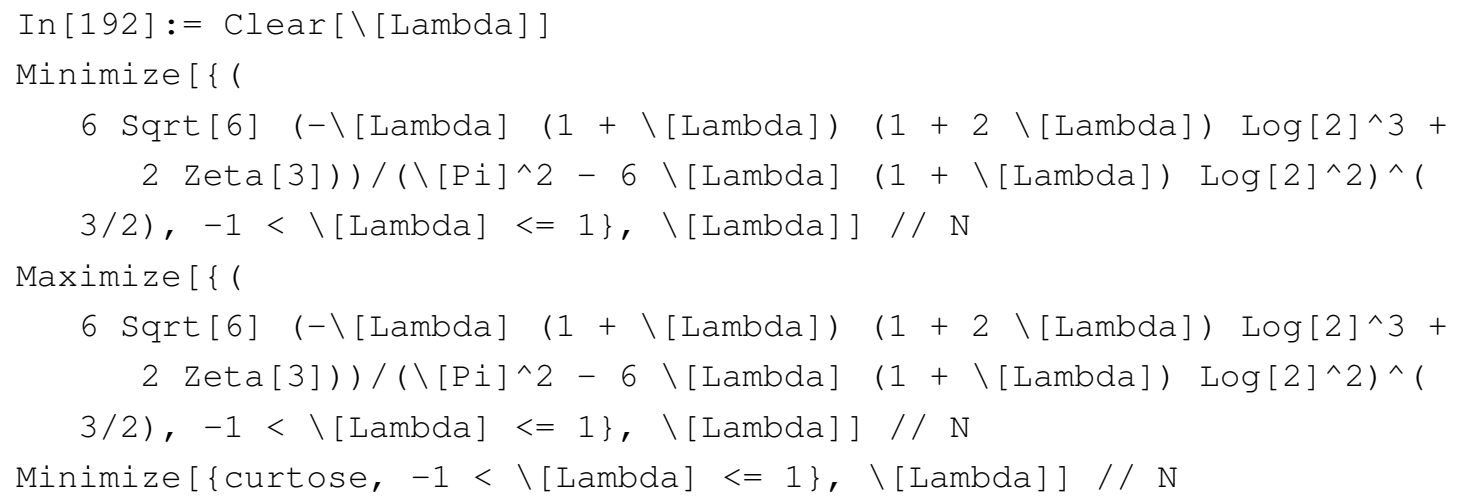




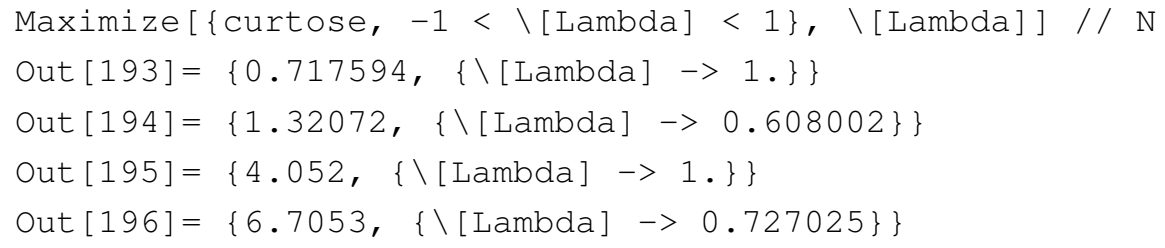

\section{F.4 Distribuição valor extremo máximo tipo I ou Gumbel generalizada}

Seja $X \sim G G u 3(\mu, \sigma, \alpha)$

$$
\begin{aligned}
\mathrm{E}(X)= & \int_{-\infty}^{\infty} x \frac{1}{\sigma}\left(1+\frac{1}{\alpha} \exp \left(-\frac{x-\mu}{\sigma}\right)\right)^{-\alpha-1} \exp \left(-\frac{x-\mu}{\sigma}\right) d x \\
= & \int_{0}^{\infty}(\mu-\sigma \ln y)\left(1+\frac{1}{\alpha} y\right)^{-\alpha-1} d y \\
= & \mu-\sigma(-\mathcal{E}+\ln (\alpha)-\psi(\alpha)), \\
E\left((X-E(X))^{n}\right)= & \int_{-\infty}^{\infty}(x-E(X))^{n} \frac{1}{\sigma}\left(1+\frac{1}{\alpha} \exp \left(-\frac{x-\mu}{\sigma}\right)\right)^{-\alpha-1} \exp \left(-\frac{x-\mu}{\sigma}\right) d x \\
= & \int_{0}^{\infty}(-\sigma \ln y+\sigma(-\mathcal{E}+\ln (\alpha)-\psi(\alpha)))^{n}\left(1+\frac{1}{\alpha} y\right)^{-\alpha-1} d y \\
= & \sigma^{n} \int_{0}^{\infty}(-\mathcal{E}+\ln (\alpha)-\psi(\alpha)-\ln y)^{n}\left(1+\frac{1}{\alpha} y\right)^{-\alpha-1} d y, \\
& \operatorname{var}(X)=E\left((X-E(X))^{2}\right)=\sigma^{2}\left(\pi^{2} / 6+\psi^{\prime}(\alpha)\right), \\
\gamma_{1, \mathrm{GGu} 3}= & \frac{E\left((X-E(X))^{3}\right)}{\left[E\left((X-E(X))^{2}\right)\right]^{3 / 2}}=\frac{\psi^{\prime \prime}(\alpha)+2 \zeta(3)}{\left(\pi^{2} / 6+\psi^{\prime}(\alpha)\right)^{3 / 2}}=\frac{\psi^{\prime \prime}(\alpha)-\psi^{\prime \prime}(1)}{\left(\psi^{\prime}(1)+\psi^{\prime}(\alpha)\right)^{3 / 2},}
\end{aligned}
$$

e

$$
\begin{aligned}
\gamma_{2, \mathrm{GGu} 3} & =\frac{E\left((X-E(X))^{4}\right)}{\left[E\left((X-E(X))^{2}\right)\right]^{2}}=\frac{3 \pi^{4} / 20+\pi^{2} \psi^{\prime}(\alpha)+3\left(\psi^{\prime}(\alpha)\right)^{2}+\psi^{\prime \prime \prime}(\alpha)}{\left(\pi^{2} / 6+\psi^{\prime}(\alpha)\right)^{2}} \\
& =\frac{3 \pi^{4}(1 / 20-1 / 36)+3\left(\pi^{2} / 6+\psi^{\prime}(\alpha)\right)^{2}+\psi^{\prime \prime \prime}(\alpha)}{\left(\pi^{2} / 6+\psi^{\prime}(\alpha)\right)^{2}}=\frac{\psi^{\prime \prime \prime}(1)+\psi^{\prime \prime \prime}(\alpha)}{\left(\psi^{\prime}(1)+\psi^{\prime}(\alpha)\right)^{2}}+3
\end{aligned}
$$

usando a transformação $y=\exp (-(x-\mu) / \sigma)$ e os resultados apresentados abaixo, obtidos com o software Mathematica Wolfram Research (2012).

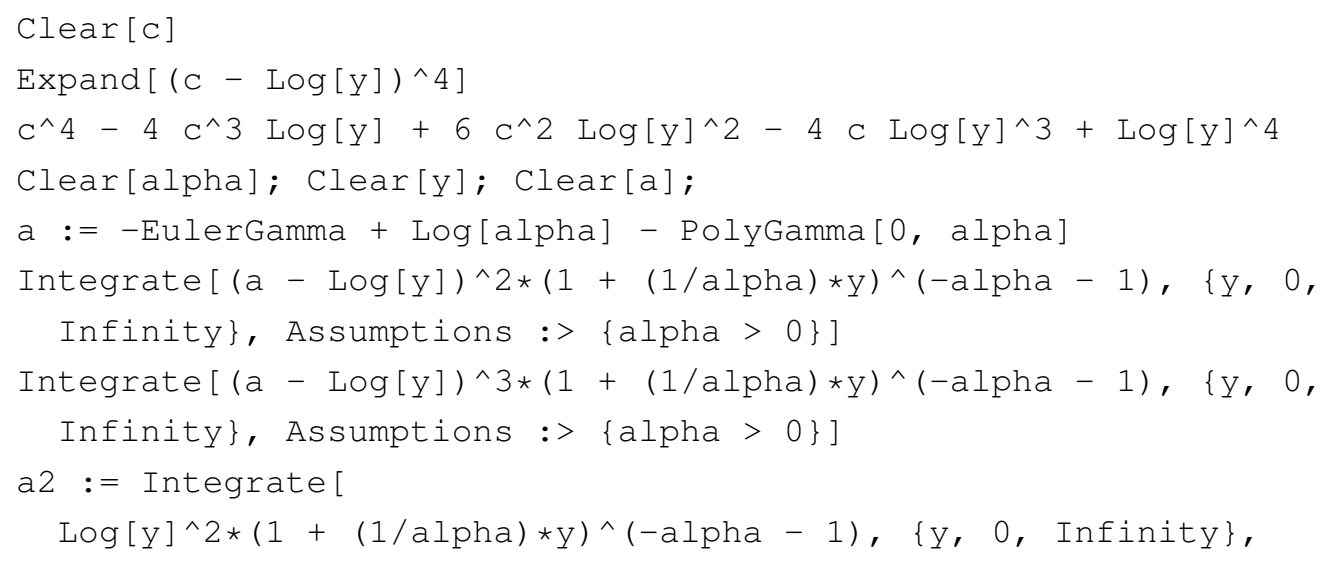




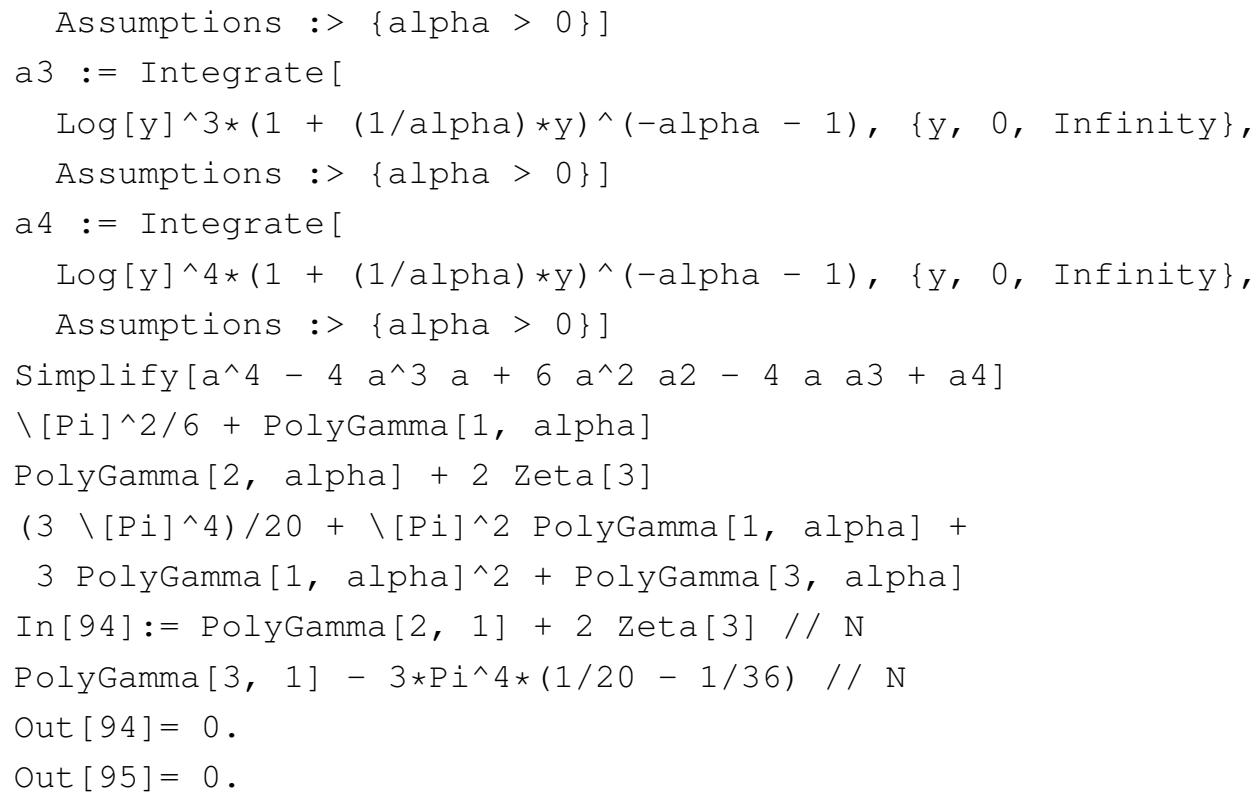

Os limites dos coeficientes de assimetria e de curtose são obtidos com o software Mathematica Wolfram Research (2012) como segue:

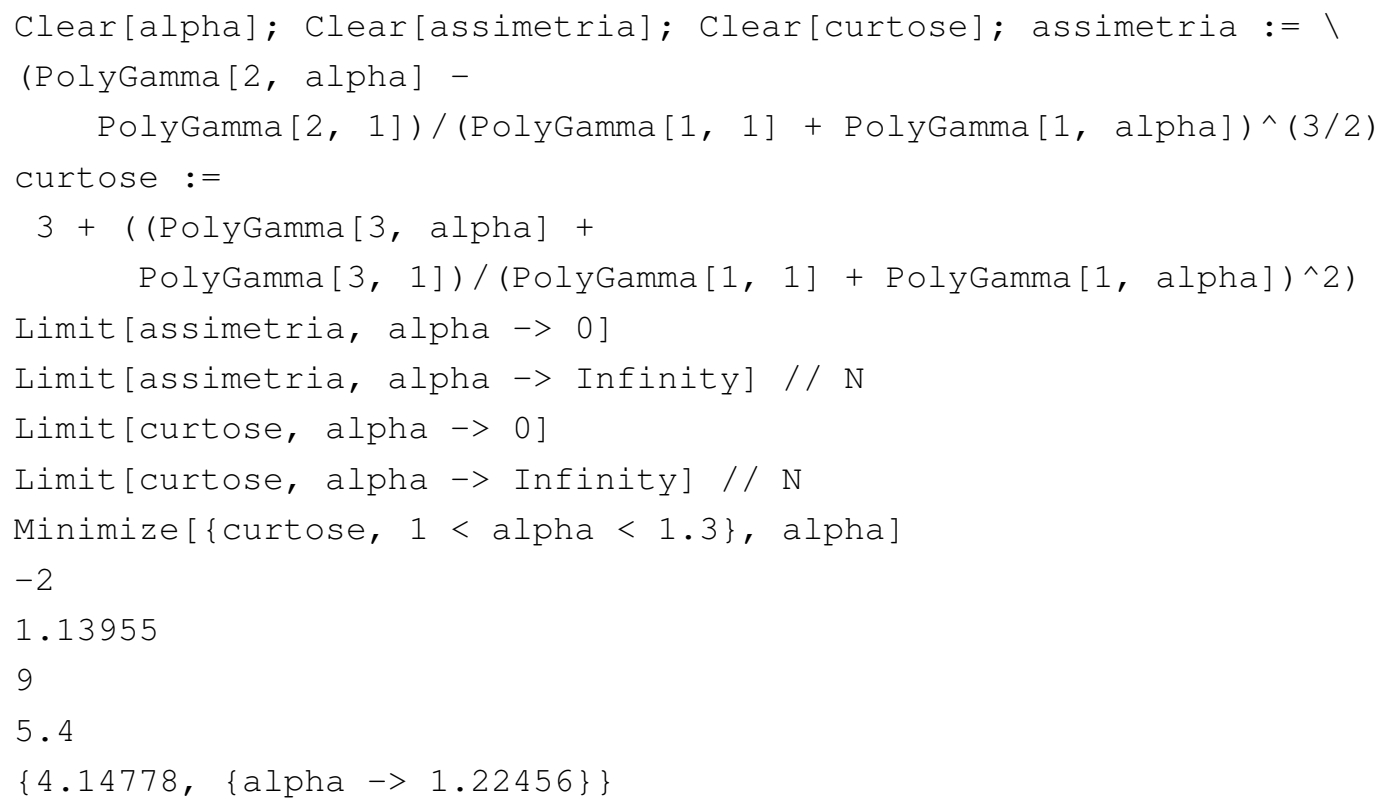

\section{F.5 Distribuição exponencial-gama com três parâmetros}

Seja $X \sim \operatorname{Exp} \operatorname{Gama3}(\mu, \sigma, \alpha)$, a função de distribuição acumulada é dada por

$$
\begin{aligned}
F_{\text {ExpGama3 }}(x) & =\int_{-\infty}^{x} \frac{1}{\sigma} \frac{1}{\Gamma(\alpha)} \exp \left(-\alpha \frac{y-\mu}{\sigma}\right) \exp \left(-\exp \left(-\frac{y-\mu}{\sigma}\right)\right) d y \\
& =\frac{1}{\Gamma(\alpha)} \int_{\exp (-(x-\mu) / \sigma)}^{\infty} w^{\alpha-1} \exp (-w) d w \\
& =\frac{\Gamma(\alpha, \exp (-(x-\mu) / \sigma))}{\Gamma(\alpha)},
\end{aligned}
$$


pois

In $[73]:=$ Clear $[$ alpha $]$

Clear $[\mathrm{c}]$

Integrate $\left[\mathrm{w}^{\wedge}(a)\right.$ pha -1$) \operatorname{Exp}[-\mathrm{w}],\{w, c, \operatorname{Infinity}\}$, Assumptions :> \{alpha $>0\}]$

Out $[75]=$ Gamma [alpha, c $]$

A esperança, variância e coeficientes de assimetria e de curtose são dadas por

$$
\begin{aligned}
\mathrm{E}(X)= & \int_{-\infty}^{\infty} x \frac{1}{\sigma} \frac{1}{\Gamma(\alpha)} \exp \left(-\alpha \frac{x-\mu}{\sigma}\right) \exp \left(-\exp \left(-\frac{x-\mu}{\sigma}\right)\right) d x \\
= & \frac{1}{\Gamma(\alpha)} \int_{0}^{\infty}(\mu-\sigma \ln y) y^{\alpha-1} \exp (-y) d y \\
= & \mu-\sigma \psi(\alpha), \\
E\left((X-E(X))^{n}\right)= & \int_{-\infty}^{\infty}(x-E(X))^{n} \frac{1}{\sigma} \frac{1}{\Gamma(\alpha)} \exp \left(-\alpha \frac{x-\mu}{\sigma}\right) \exp \left(-\exp \left(-\frac{x-\mu}{\sigma}\right)\right) d x \\
= & \sigma^{n} \frac{1}{\Gamma(\alpha)} \int_{0}^{\infty}(\psi(\alpha)-\ln y)^{n} y^{\alpha-1} \exp (-y) d y \\
& \operatorname{var}(X)=E\left((X-E(X))^{2}\right)=\sigma^{2} \psi^{\prime}(\alpha), \\
& \gamma_{1, \text { ExpGama3 }}=\frac{E\left((X-E(X))^{3}\right)}{\left[E\left((X-E(X))^{2}\right)\right]^{3 / 2}}=\frac{-\psi^{\prime \prime}(\alpha)}{\psi^{\prime}(\alpha)^{3 / 2}},
\end{aligned}
$$

$\mathrm{e}$

$$
\gamma_{2, \text { ExpGama3 } 3}=\frac{E\left((X-E(X))^{4}\right)}{\left[E\left((X-E(X))^{2}\right)\right]^{2}}=\frac{\psi^{\prime \prime \prime}(\alpha)}{\psi^{\prime}(\alpha)^{2}}+3,
$$

usando a transformação $y=\exp (-(x-\mu) / \sigma)$ e os resultados apresentados abaixo, obtidos com o software Mathematica Wolfram Research (2012).

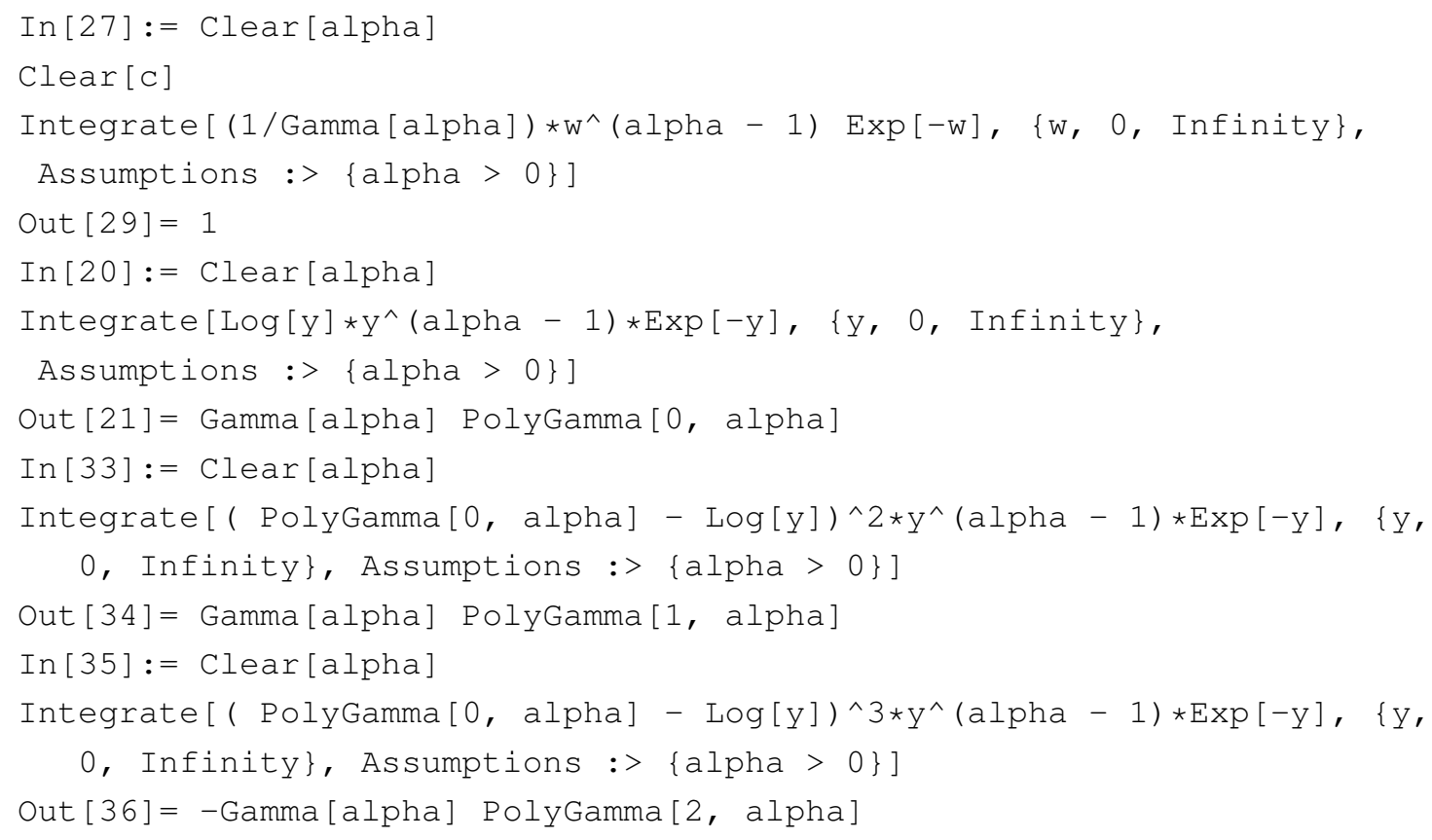




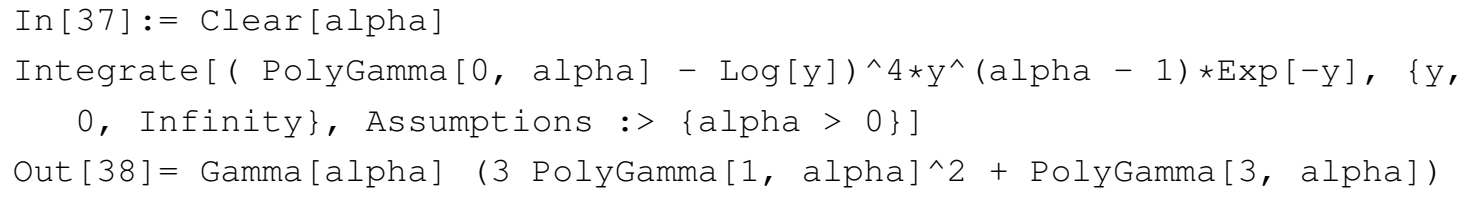

Os limites dos coeficientes de assimetria e de curtose são obtidos com o software Mathematica Wolfram Research (2012) como segue:

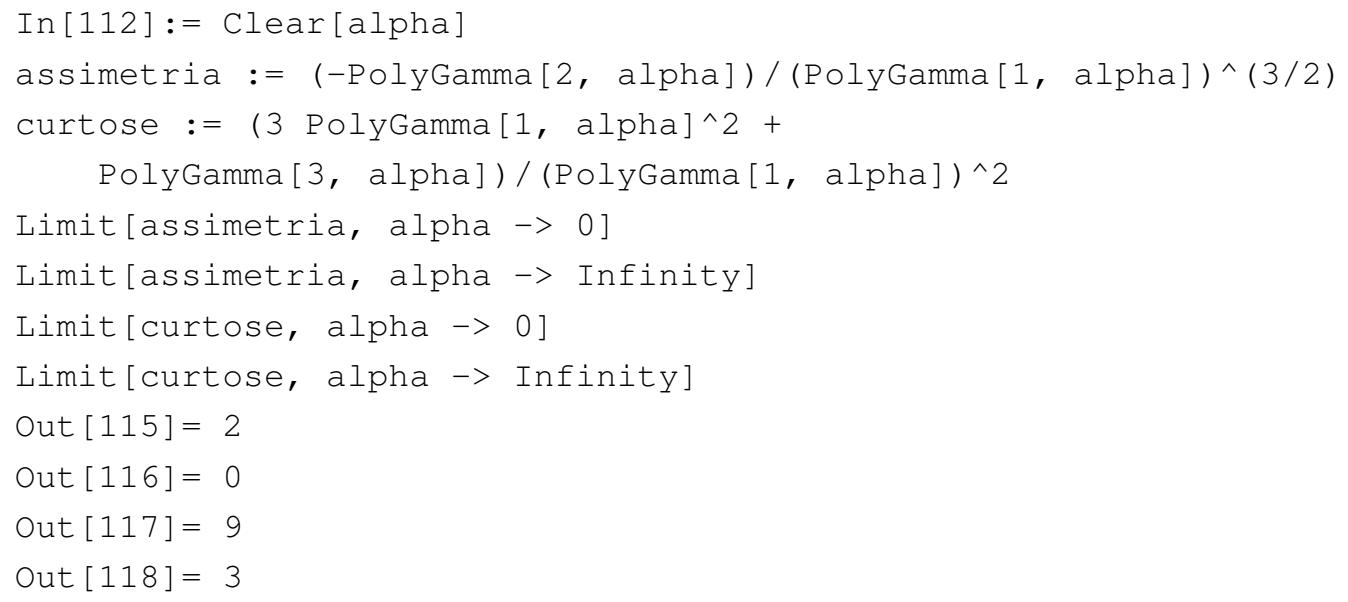

\section{F.6 Distribuição exponencial-gama}

Seja $X \sim \operatorname{Exp} \operatorname{Gama}(\mu, \sigma, \alpha, \beta)$, a função de distribuição acumulada é dada por

$$
\begin{aligned}
& F_{E x p G a m a}(x ; \mu, \sigma, \alpha, \beta)=\int_{-\infty}^{x} \frac{\alpha^{\beta}}{\sigma \Gamma(\beta)} \exp \left(-\alpha \exp \left(-\frac{t-\mu}{\sigma}\right)\right) \exp \left(-\beta \frac{t-\mu}{\sigma}\right) d t \\
= & \int_{\infty}^{\alpha \exp (-(x-\mu) / \sigma)} \frac{\alpha^{\beta}}{\sigma \Gamma(\beta)} \exp (-y)\left(\frac{y}{\alpha}\right)^{\beta}\left(-\frac{\sigma}{y} d y\right)=\frac{1}{\Gamma(\beta)} \int_{\alpha \exp (-(x-\mu) / \sigma)}^{\infty} \exp (-y) y^{\beta-1} d y \\
= & \frac{\Gamma\left(\beta, \alpha \exp \left(-\frac{x-\mu}{\sigma}\right)\right)}{\Gamma(\beta)}
\end{aligned}
$$

em que consideramos a transformação $y=\alpha \exp (-(t-\mu) / \sigma)$ e $\Gamma(a, x)=\int_{x}^{\infty} \exp (-t) t^{a-1} d t$ é a função gama incompleta (ver Grashteyn \& Ryzhik (2000) equação 8.350.2).

\section{F.7 Distribuição logística generalizada tipo IV}

A função de distribuição acumulada é dada por

$$
\begin{aligned}
F(x) & =\int_{-\infty}^{x}\left(\frac{\alpha}{\beta}\right)^{\alpha} \frac{1}{\sigma B(\alpha, \beta)} \frac{[\exp (-(y-\mu) / \sigma)]^{\alpha}}{[1+(\alpha / \beta) \exp (-(y-\mu) / \sigma)]^{\alpha+\beta}} d y \\
& =\int_{-\infty}^{(x-\mu) / \sigma}\left(\frac{\alpha}{\beta}\right)^{\alpha} \frac{1}{B(\alpha, \beta)} \frac{[\exp (-z)]^{\alpha}}{[1+(\alpha / \beta) \exp (-z)]^{\alpha+\beta}} d z \\
& =\left(\frac{1}{\alpha(1+(\alpha / \beta) \exp (-(x-\mu) / \sigma))}\right)^{\beta}\left(\alpha+\beta \exp ((x-\mu) / \sigma)^{\beta}\right. \\
& =\frac{1}{\beta B(\alpha, \beta)}(\beta / \alpha) \exp ((x-\mu) / \sigma)^{\beta}{ }_{2} F_{1}(\beta, \alpha+\beta ; 1+\beta ;-(\beta / \alpha) \exp ((x-\mu) / \sigma)) \frac{1}{\beta B(\alpha, \beta)}
\end{aligned}
$$


de acordo com os resultados apresentados abaixo, obtidos com o software Mathematica Wolfram Research (2012).

$$
\begin{aligned}
& \text { In [12]:= Clear[z]; Clear[alpha];Clear[beta] } \\
& \text { Integrate [(1/Beta[alpha, beta])*(alpha/beta)^ } \\
& \text { alpha* }\left(\operatorname{Exp}[-z]^{\wedge}\right. \\
& \text { alpha) /( }(1+(\text { alpha/beta }) * \operatorname{Exp}[-z])^{\wedge}(\text { alpha }+ \\
& \text { beta)), }\{z,- \text { Infinity, y\}, Assumptions :> \{alpha }>0 \text {, beta }>0\} \text { ] } \\
& \text { Out }[15]=\left(\left(\text { alpha }\left(1+\left(\text { alpha } E^{\wedge}-y\right) / \text { beta }\right)\right)^{\wedge} \text {-beta }(1 /(\right. \\
& \text { alpha + beta } \left.\left.E^{\wedge} \mathrm{Y}\right)\right)^{\wedge} a l p h a\left(a l p h a+\text { beta } E^{\wedge} \mathrm{Y}\right) \wedge(a l p h a+\text { beta) } \\
& \text { Hypergeometric2F1[beta, alpha + beta, } \\
& \left.\left.\left.\left.1 \text { + beta, -((beta } E^{\wedge} y\right) / a l p h a\right)\right]\right) /(\text { beta Beta[alpha, beta]) } \\
& M_{G L I V}(t)=\int_{-\infty}^{\infty} \exp (t y) \frac{1}{B(\alpha, \beta)} \frac{[(\alpha / \beta) \exp (-y)]^{\alpha}}{[1+(\alpha / \beta) \exp (-y)]^{\alpha+\beta}} d y \\
& =\frac{1}{B(\alpha, \beta)}\left(\frac{\beta}{\alpha}\right)^{-t} \int_{-\infty}^{\infty} \frac{[(\alpha / \beta) \exp (-y)]^{\alpha-t}}{[1+(\alpha / \beta) \exp (-y)]^{\alpha+\beta}} d y \\
& =\frac{1}{B(\alpha, \beta)}\left(\frac{\beta}{\alpha}\right)^{-t} \int_{0}^{\infty} \frac{w^{\alpha-t-1}}{[1+w]^{\alpha+\beta}} d w \\
& =\frac{1}{B(\alpha, \beta)}\left(\frac{\beta}{\alpha}\right)^{-t} B(\alpha-t, \beta+t) \\
& =\left(\frac{\beta}{\alpha}\right)^{-t} \frac{\Gamma(\alpha-t) \Gamma(\beta+t)}{\Gamma(\alpha) \Gamma(\beta)} \quad-\beta<t<\alpha,
\end{aligned}
$$

pois $B(x, y)=\int_{0}^{\infty}\left(t^{x-1} /(1+t)^{x+y}\right) d t$ e $B(x, y)=(\Gamma(x) \Gamma(y)) / \Gamma(x+y)$. Logo,

$$
\begin{gathered}
\mathrm{E}(Y)=\frac{d M(t)}{d t}(0)=\psi^{\prime}(\beta)-\psi^{\prime}(\alpha)-\ln \frac{\beta}{\alpha}, \\
\operatorname{var}(Y)=\left(\psi^{\prime}(\beta)+\psi^{\prime}(\alpha)\right), \\
\gamma_{1, G L I V}(Y)=\frac{\psi^{\prime \prime}(\beta)-\psi^{\prime \prime}(\alpha)}{\left(\psi^{\prime}(\beta)+\psi^{\prime}(\alpha)\right)^{3 / 2}}
\end{gathered}
$$

$\mathrm{e}$

$$
\gamma_{2, G L I V}(Y)=\frac{\psi^{\prime \prime \prime}(\beta)+\psi^{\prime \prime \prime}(\alpha)}{\left(\psi^{\prime}(\beta)+\psi^{\prime}(\alpha)\right)^{2}}+3
$$

usando as propriedades da assimetria e curtose dadas em (4.8) e e os resultados apresentados abaixo, obtidos com o software Mathematica Wolfram Research (2012).

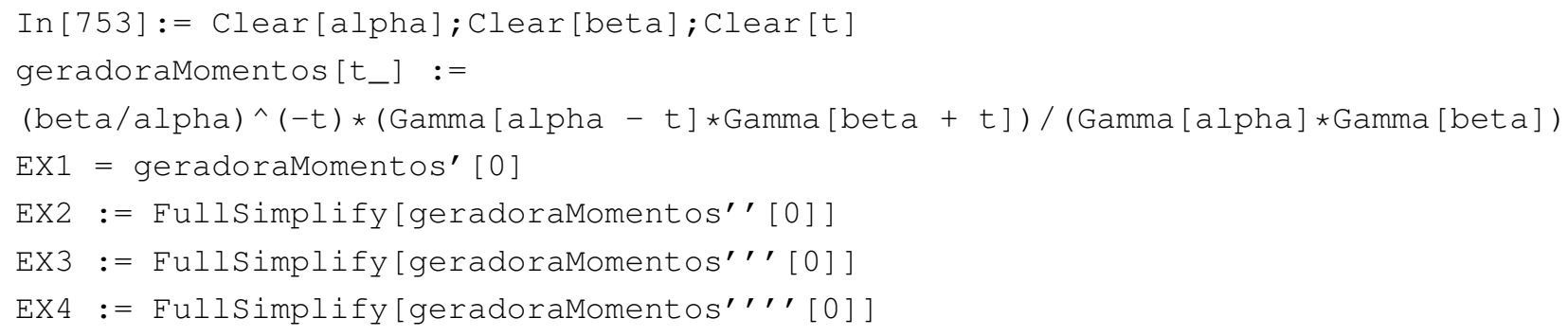




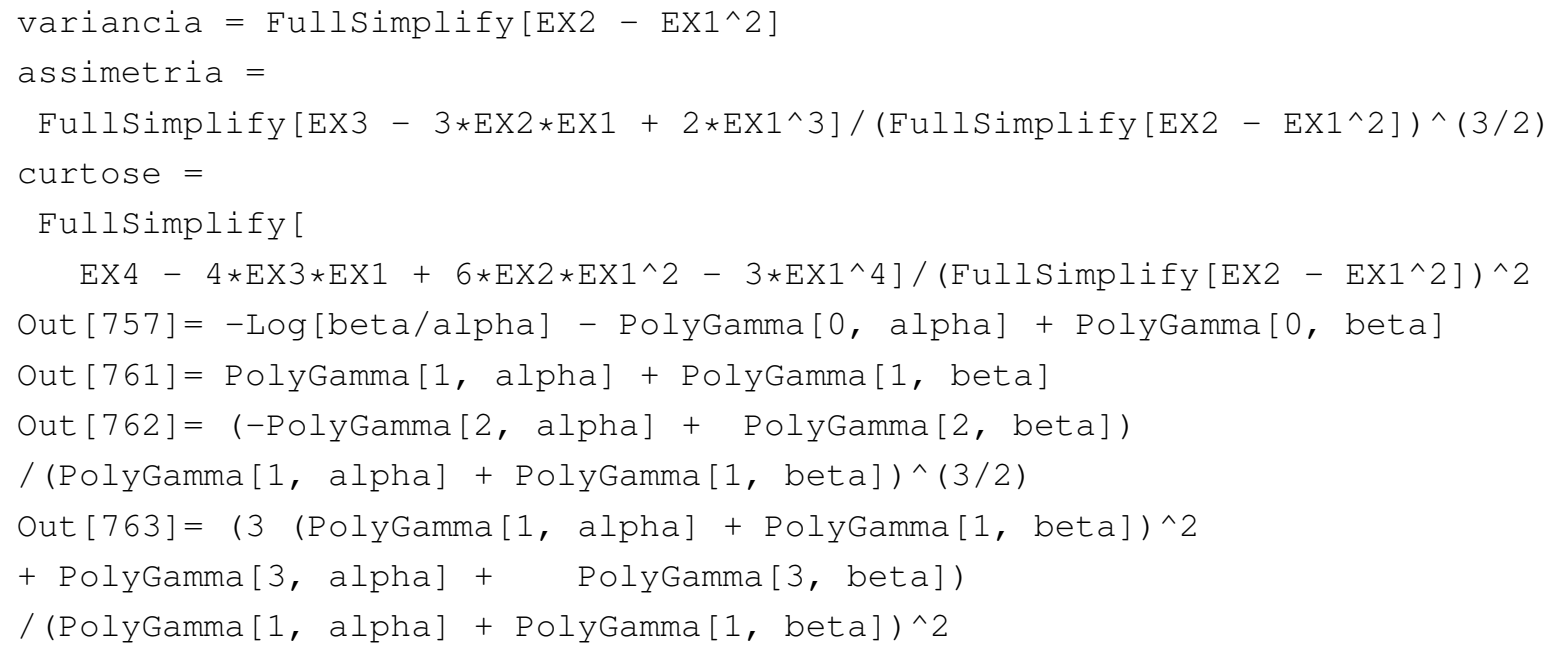

Então, se $X=\mu+\sigma Y \sim G L I V(\mu, \sigma, \alpha, \beta)$, a esperança, variância e coeficientes de assimetria e de curtose são dadas por

e

$$
\begin{gathered}
\mathrm{E}(X)=\mu+\sigma\left(\psi(\beta)-\psi(\alpha)-\ln \frac{\beta}{\alpha}\right), \\
\operatorname{var}(X)=\sigma^{2}\left(\psi^{\prime}(\beta)+\psi^{\prime}(\alpha)\right), \\
\gamma_{1, G L I V}(X)=\frac{\psi^{\prime \prime}(\beta)-\psi^{\prime \prime}(\alpha)}{\left(\psi^{\prime}(\beta)+\psi^{\prime}(\alpha)\right)^{3 / 2}}
\end{gathered}
$$

$$
\gamma_{2, G L I V}(X)=\frac{\psi^{\prime \prime \prime}(\alpha)+\psi^{\prime \prime \prime}(\beta)}{\left(\psi^{\prime}(\beta)+\psi^{\prime}(\alpha)\right)^{2}}+3
$$

Os limites dos coeficientes de assimetria e de curtose são obtidos com o software Mathematica Wolfram Research (2012) como segue:

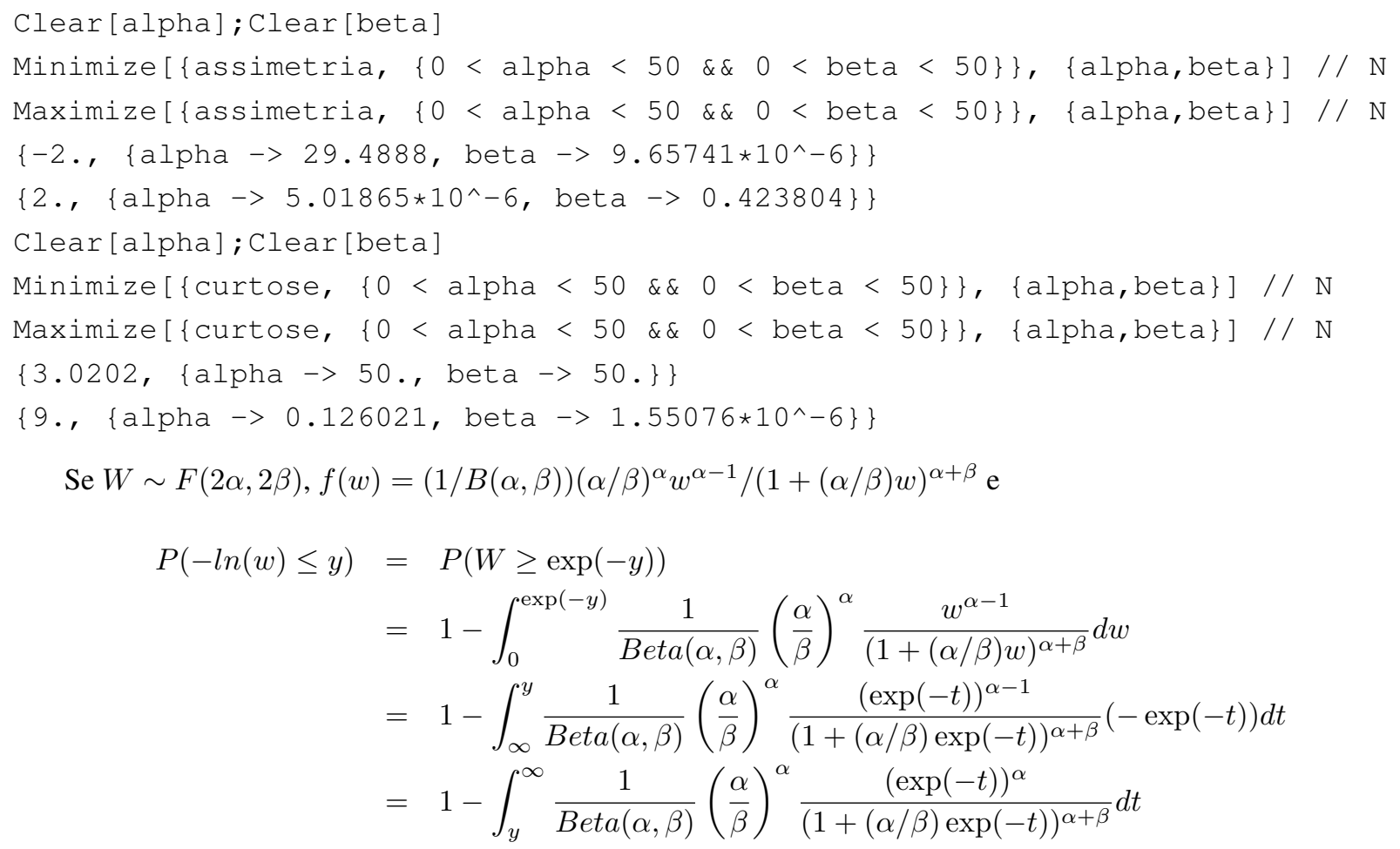




$$
=\int_{-\infty}^{y} \frac{1}{\operatorname{Beta}(\alpha, \beta)}\left(\frac{\alpha}{\beta}\right)^{\alpha} \frac{(\exp (-t))^{\alpha}}{(1+(\alpha / \beta) \exp (-t))^{\alpha+\beta}} d t
$$

então $-\ln W \sim G L I V(0,1, \alpha, \beta)$ e $\mu-\sigma \ln W \sim G L I V(\mu, \sigma, \alpha, \beta)$.

\section{F.8 Mistura de dois componentes de distribuição valor extremo máximo}

Seja $X \sim T C E V\left(\mu_{1}, \sigma_{1}, \mu_{2}, \sigma_{2}, \alpha\right)$, os coeficientes de assimetria e de curtose, assim como seus valores mínimos e máximos, podem ser obtidos com os comandos do software Mathematica Wolfram Research (2012) conforme os resultados apresentados abaixo.

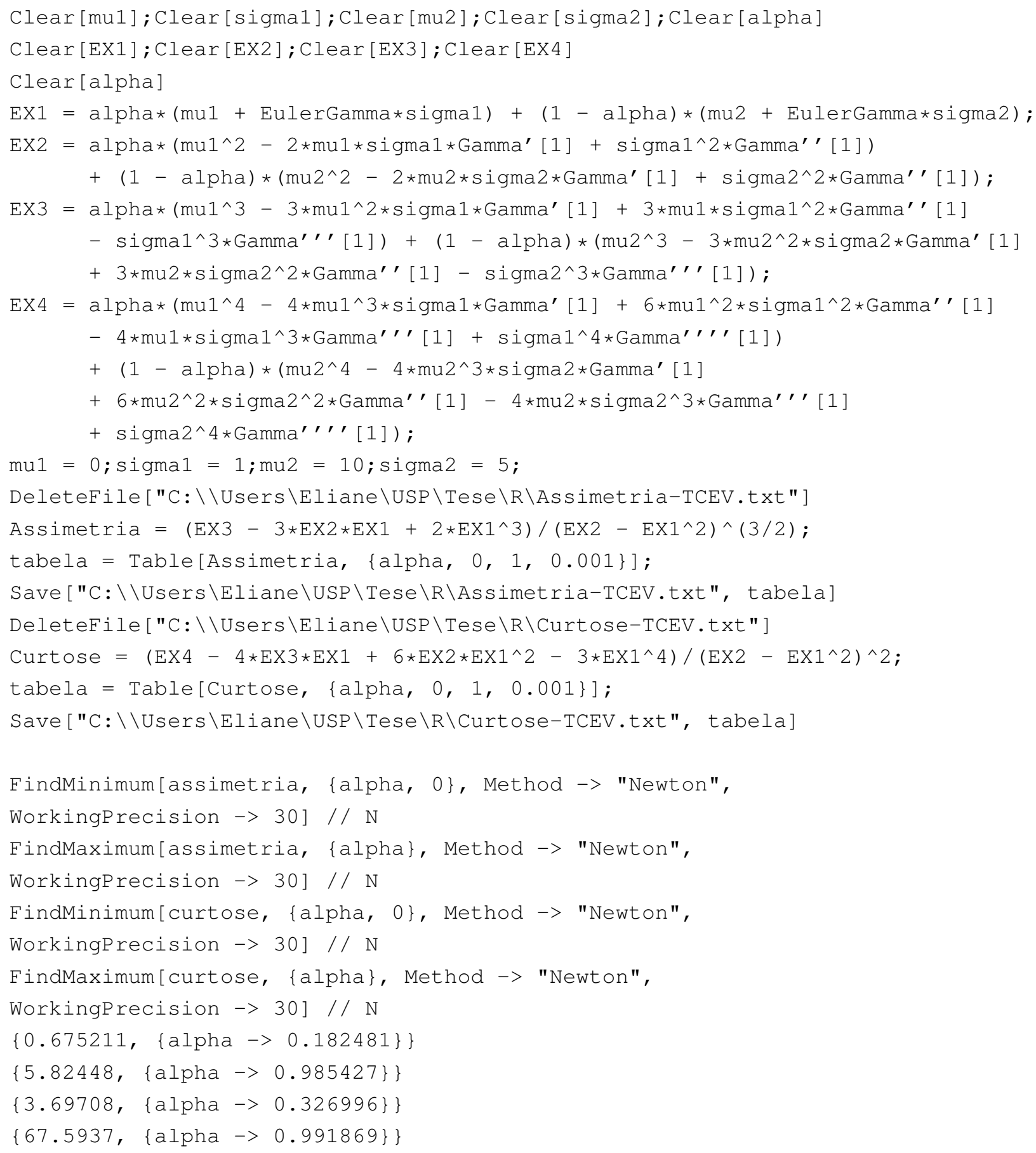




\section{Apêndice G}

\section{Índice da cauda}

Temos que

$$
\begin{gathered}
\lim _{t \rightarrow \infty} \frac{\exp \left(-\left[1+\alpha\left(\frac{t x-\mu}{\sigma}\right)\right]^{-1 / \alpha}\right)}{\exp \left(-\left[1+\alpha\left(\frac{t-\mu}{\sigma}\right)\right]^{-1 / \alpha}\right)}=1, \quad \text { se } \quad \alpha>0 \\
\lim _{t \rightarrow \infty} \frac{\exp (-\exp (-(t x-\mu) / \sigma))}{\exp (-\exp (-(t-\mu) / \sigma))}=1,
\end{gathered}
$$

$\mathrm{e}$

$$
\lim _{t \rightarrow \infty} \frac{\exp (-(t x-\mu) / \sigma)}{\exp (-(t-\mu) / \sigma)}=\left\{\begin{array}{ccc}
\infty & \text { se } & 0<x<1 \\
0 & \text { se } & x>1
\end{array}\right.
$$

conforme obtido com o software Mathematica Wolfram Research (2012):

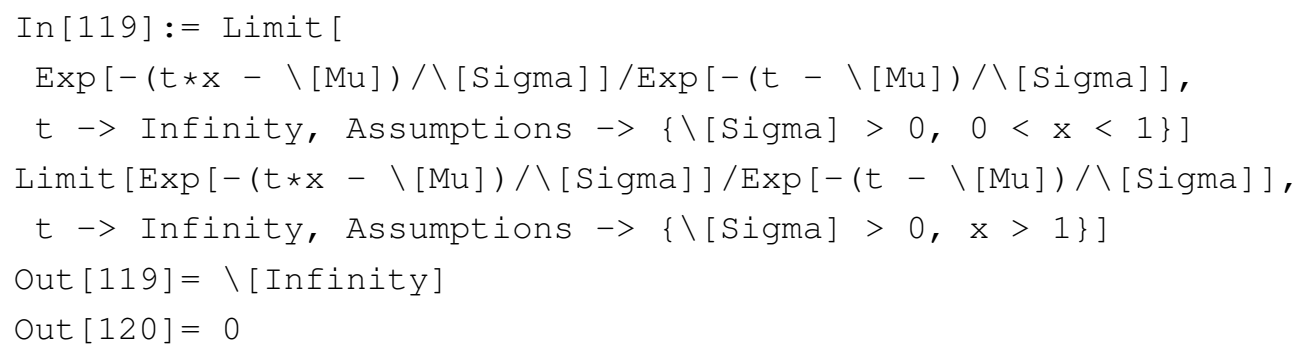

Apresentamos, a seguir, o índice de variação regular e/ou o índice da cauda de algumas distribuições que são generalizações da distribuição valor extremo máximo ou Gumbel.

Usando a regra de L'Hopital, temos que

$$
\lim _{t \rightarrow \infty} \frac{\bar{F}(t x)}{\bar{F}(t)}=\lim _{t \rightarrow \infty} \frac{1-F(t x)}{1-F(t)}=\lim _{t \rightarrow \infty} \frac{x f(t x)}{f(t)}=x \lim _{t \rightarrow \infty} \frac{f(t x)}{f(t)}
$$

\section{G.1 Distribuição valor extremo generalizada}

Seja $X \sim G E V(\mu, \sigma, \alpha)$ tal que $\alpha>0$, por (G.1), temos que

$$
\begin{aligned}
\lim _{t \rightarrow \infty} \frac{\bar{F}_{G E V}(t x)}{\bar{F}_{G E V}(t)} & =x \lim _{t \rightarrow \infty} \frac{\frac{1}{\sigma} \exp \left(-\left[1+\alpha\left(\frac{t x-\mu}{\sigma}\right)\right]^{-1 / \alpha}\right)\left[1+\alpha\left(\frac{t x-\mu}{\sigma}\right)\right]^{-1 / \alpha-1}}{\frac{1}{\sigma} \exp \left(-\left[1+\alpha\left(\frac{t-\mu}{\sigma}\right)\right]^{-1 / \alpha}\right)\left[1+\alpha\left(\frac{t-\mu}{\sigma}\right)\right]^{-1 / \alpha-1}} \\
& =x 1 x^{-1 / \alpha-1}=x^{-1 / \alpha},
\end{aligned}
$$


isto é, a distribuição valor extremo generalizada tipo II ou família Fréchet é de variação regular com índice de variação $-1 / \alpha$ e índice da cauda $\alpha$. Se $\alpha=0$, por (G.2) e (G.3), temos que

$$
\begin{aligned}
\lim _{t \rightarrow \infty} \frac{\bar{F}_{E V}(t x)}{\bar{F}_{E V}(t)} & =x \lim _{t \rightarrow \infty} \frac{(1 / \sigma) \exp (-\exp (-(t x-\mu) / \sigma)) \exp (-(t x-\mu) / \sigma)}{(1 / \sigma) \exp (-\exp (-(t-\mu) / \sigma)) \exp (-(t-\mu) / \sigma)} \\
& =\left\{\begin{array}{lll}
\infty & \text { se } & 0<x<1 \\
1 & \text { se } & x=1 \\
0 & \text { se } & x>1 .
\end{array}\right.
\end{aligned}
$$

isto é, a distribuição valor extremo máximo ou Gumbel é de variação rápida ou variação regular com índice de variação $-\infty$.

\section{G.2 Distribuição Gumbel exponencializada}

Seja $X \sim E G u(\mu, \sigma, \alpha)$ tal que $\alpha>0$, por (G.2) e (G.3), temos que

$$
\begin{aligned}
\lim _{t \rightarrow \infty} \frac{\bar{F}_{E G u}(t x)}{\bar{F}_{E G u}(t)} & =\lim _{t \rightarrow \infty} \frac{1-\left(1-\left[1-\exp \left(-\exp \left(-\frac{t x-\mu}{\sigma}\right)\right)\right]^{\alpha}\right)}{1-\left(1-\left[1-\exp \left(-\exp \left(-\frac{t-\mu}{\sigma}\right)\right)\right]^{\alpha}\right)} \\
& =\lim _{t \rightarrow \infty}\left(\frac{-\exp \left(-\exp \left(-\frac{t x-\mu}{\sigma}\right)\right)\left(-\exp \left(-\frac{t x-\mu}{\sigma}\right)\right) \frac{-x}{\sigma}}{-\exp \left(-\exp \left(-\frac{t-\mu}{\sigma}\right)\right)\left(-\exp \left(-\frac{t-\mu}{\sigma}\right)\right) \frac{-1}{\sigma}}\right)^{\alpha} \\
& =\left\{\begin{array}{ccc}
\infty & \text { se } & 0<x<1 \\
1 & \text { se } & x=1 \\
0 & \text { se } & x>1 .
\end{array}\right.
\end{aligned}
$$

isto é, a distribuição Gumbel exponencializada é de variação rápida ou variação regular com índice de variação $-\infty$.

\section{G.3 Distribuição valor extremo transmutada}

Seja $X \sim T E V(\mu, \sigma, \alpha)$ tal que $-1<\alpha \leq 1$, por (G.2) e (G.3) temos que

$$
\begin{aligned}
& \lim _{t \rightarrow \infty} \frac{\bar{F}_{T E V}(t x)}{\bar{F}_{T E V}(t)}=\lim _{t \rightarrow \infty} \frac{x \frac{1}{\sigma} \exp \left[-\frac{t x-\mu}{\sigma}-\exp \left(-\frac{t x-\mu}{\sigma}\right)\right]\left[1+\alpha-2 \alpha \exp \left(-\exp \left(-\frac{t x-\mu}{\sigma}\right)\right)\right]}{\frac{1}{\sigma} \exp \left[-\frac{t-\mu}{\sigma}-\exp \left(-\frac{t-\mu}{\sigma}\right)\right]\left[1+\alpha-2 \alpha \exp \left(-\exp \left(-\frac{t-\mu}{\sigma}\right)\right)\right]} \\
= & \lim _{t \rightarrow \infty} x \frac{\exp \left[-\frac{t x-\mu}{\sigma}\right]}{\exp \left[-\frac{t-\mu}{\sigma}\right]} \lim _{t \rightarrow \infty} \frac{\exp \left[-\exp \left(-\frac{t x-\mu}{\sigma}\right)\right]}{\exp \left[-\exp \left(-\frac{t-\mu}{\sigma}\right)\right]} \lim _{t \rightarrow \infty} \frac{\left[1+\alpha-2 \alpha \exp \left(-\exp \left(-\frac{t x-\mu}{\sigma}\right)\right)\right]}{\left[1+\alpha-2 \alpha \exp \left(-\exp \left(-\frac{t-\mu}{\sigma}\right)\right)\right]} \\
= & \left\{\begin{array}{lll}
\infty & \text { se } & 0<x<1 \\
1 & \text { se } & x=1 \\
0 & \text { se } & x>1 .
\end{array}\right.
\end{aligned}
$$

isto é, a distribuição valor extremo transmutada é de variação rápida ou variação regular com índice de variação $-\infty$. 


\section{G.4 Distribuição valor extremo máximo tipo I ou Gumbel generalizada com três parâme-} tros

Seja $X \sim G G u 3(\mu, \sigma, \alpha)$ tal que $\alpha>0$, por (G.3) temos que

$$
\lim _{t \rightarrow \infty} \frac{\bar{F}_{G G u 3}(t x)}{\bar{F}_{G G u 3}(t)}=\lim _{t \rightarrow \infty} x \frac{\frac{1}{\sigma}\left(1+\frac{1}{\alpha} \exp \left(-\frac{t x-\mu}{\sigma}\right)\right)^{-\alpha-1} \exp \left(-\frac{t x-\mu}{\sigma}\right)}{\frac{1}{\sigma}\left(1+\frac{1}{\alpha} \exp \left(-\frac{t-\mu}{\sigma}\right)\right)^{-\alpha-1} \exp \left(-\frac{t-\mu}{\sigma}\right)}=\left\{\begin{array}{ccc}
\infty & \text { se } & 0<x<1 \\
1 & \text { se } & x=1 \\
0 & \text { se } & x>1
\end{array}\right.
$$

isto é, a distribuição Gumbel generalizada com três parâmetros é de variação rápida ou variação regular com índice de variação $-\infty$.

\section{G.5 Distribuição exponencial-gama com três parâmetros}

Seja $X \sim \operatorname{Exp} \operatorname{Gama} 3(\mu, \sigma, \alpha)$ tal que $\alpha>0$, por (G.2) e (G.3) temos que

$$
\lim _{t \rightarrow \infty} \frac{\bar{F}_{\text {ExpGama3 }}(t x)}{\bar{F}_{\text {ExpGama3 }}(t)}=\lim _{t \rightarrow \infty} x \frac{\frac{1}{\Gamma(\alpha)} \frac{1}{\sigma} \exp \left(-\exp \left(-\frac{t x-\mu}{\sigma}\right)\right) \exp \left(-\alpha \frac{t x-\mu}{\sigma}\right)}{\frac{1}{\Gamma(\alpha)} \frac{1}{\sigma} \exp \left(-\exp \left(-\frac{t-\mu}{\sigma}\right)\right) \exp \left(-\alpha \frac{t-\mu}{\sigma}\right)}=\left\{\begin{array}{ccc}
\infty & \text { se } & 0<x<1 \\
1 & \text { se } & x=1 \\
0 & \text { se } & x>1 .
\end{array}\right.
$$

isto é, a distribuição exponencial-gama com três parâmetros é de variação rápida ou variação regular com índice de variação $-\infty$.

\section{G.6 Distribuição logística generalizada tipo IV}

Seja $X \sim G L I V(\mu, \sigma, \alpha, \beta)$, por (G.3) temos que

$$
\begin{aligned}
& \lim _{t \rightarrow \infty} \frac{\bar{F}_{G L I V}(t x)}{\bar{F}_{G L I V}(t)}=x \lim _{t \rightarrow \infty} \frac{\left(\frac{\alpha}{\beta}\right)^{\alpha} \frac{1}{\sigma B(\alpha, \beta)} \frac{[\exp (-(t x-\mu) / \sigma)]^{\alpha}}{\left(\frac{\alpha}{\beta}\right)^{\alpha} \frac{1}{\sigma B(\alpha, \beta)} \frac{[\exp (-(t x-\mu) / \sigma)]^{\alpha+\beta}}{\left[1+\frac{\alpha}{\beta} \exp (-(t-\mu) / \sigma)\right]^{\alpha+\beta}}}}{=x \lim _{t \rightarrow \infty} \frac{[\exp (-(t x-\mu) / \sigma)]^{\alpha}}{[\exp (-(t-\mu) / \sigma)]^{\alpha}} \frac{\left[1+\frac{\alpha}{\beta} \exp (-(t-\mu) / \sigma)\right]^{\alpha+\beta}}{\left[1+\frac{\alpha}{\beta} \exp (-(t x-\mu) / \sigma)\right]^{\alpha+\beta}}} \\
& =\left\{\begin{array}{ccc}
\infty & \text { se } & 0<x<1 \\
1 & \text { se } & x=1 \\
0 & \text { se } & x>1,
\end{array}\right.
\end{aligned}
$$

isto é, a distribuição logística generalizada tipo IV é de variação rápida ou variação regular com índice de variação $-\infty$.

\section{G.7 Mistura de dois componentes de distribuição valor extremo máximo}

Seja $X \sim T C E V\left(\mu, \sigma, \alpha, \mu^{*}, \sigma^{*}\right)$, tal que $0<\alpha<0,5$ temos que

$$
\lim _{t \rightarrow \infty} \frac{\bar{F}_{T C E V}(t x)}{\bar{F}_{T C E V}(t)}
$$




$$
\begin{aligned}
& =\lim _{t \rightarrow \infty} x \frac{\frac{(1+\alpha)}{\sigma} \exp \left(-\frac{t x-\mu}{\sigma}\right) \exp \left(-\exp \left(-\frac{t x-\mu}{\sigma}\right)\right)-\frac{\alpha}{\sigma^{*}} \exp \left(-\frac{t x-\mu^{*}}{\sigma^{*}}\right) \exp \left(-\exp \left(-\frac{t x-\mu^{*}}{\sigma^{*}}\right)\right)}{\frac{(1+\alpha)}{\sigma} \exp \left(-\frac{t-\mu}{\sigma}\right) \exp \left(-\exp \left(-\frac{t-\mu}{\sigma}\right)\right)-\frac{\alpha}{\sigma^{*}} \exp \left(-\frac{t-\mu^{*}}{\sigma^{*}}\right) \exp \left(-\exp \left(-\frac{t-\mu^{*}}{\sigma^{*}}\right)\right)} \\
& =\left\{\begin{array}{llc}
\infty & \text { se } & 0<x<1 \\
1 & \text { se } & x=1 \\
0 & \text { se } & x>1,
\end{array}\right.
\end{aligned}
$$

conforme obtido com o software Mathematica Wolfram Research (2012):

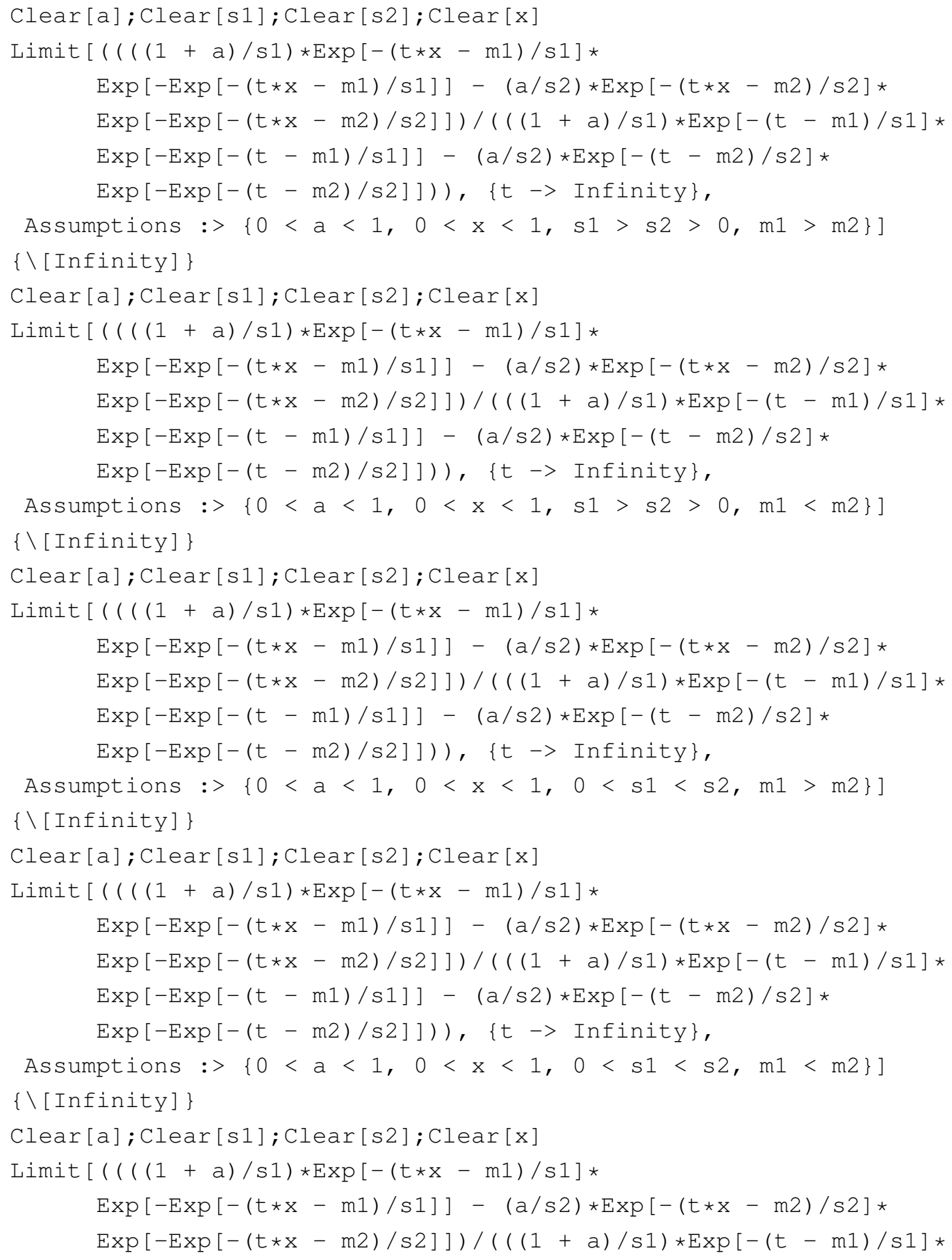




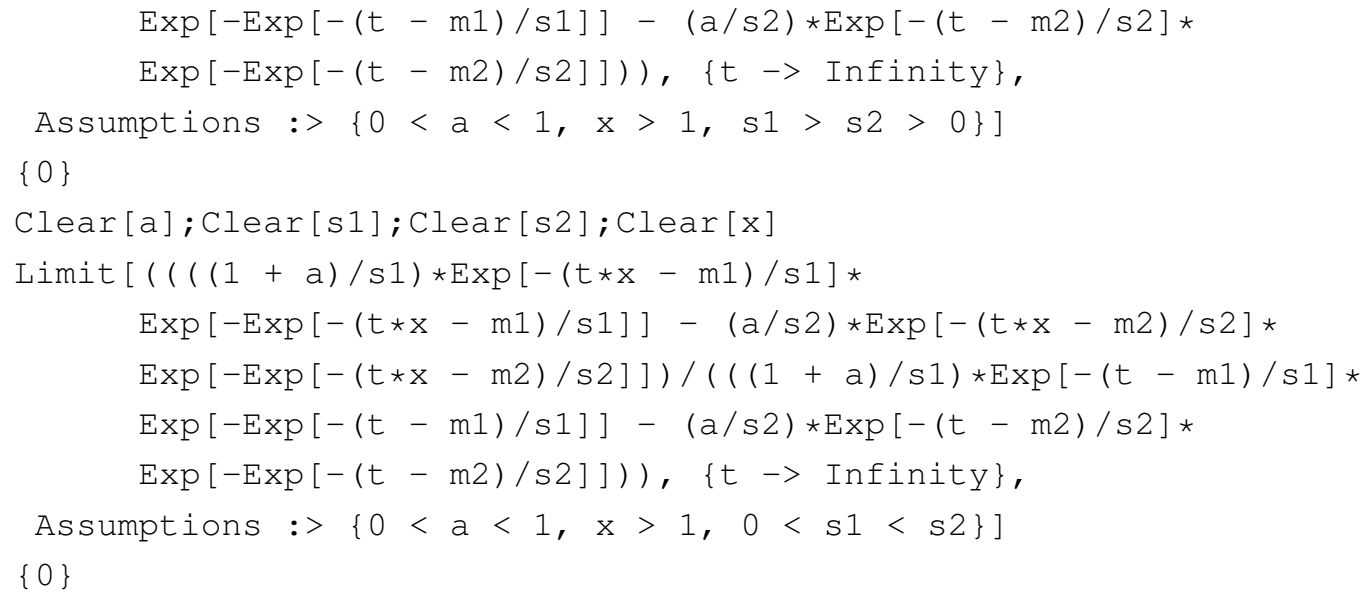

isto é, a mistura de dois componentes de distribuição valor extremo máximo é de variação rápida ou variação regular com índice de variação $-\infty$. 


\section{Referências Bibliográficas}

Adeyemi, S., \& Ojo, M. O. (2003). A generalization of the Gumbel Distribution. Kragujevac J. Math, 25, 19-29.

Aitkin, M. \& Rubin, D. B. (1985). Estimation and hypothesis testing in finite mixture models. Journal of the Royal Statistical Society B,47, 67-75.

Akaike, H. (1973). Information theory and an extension of the maximum likelihood principle. Second International Symposium on Information Theory, 267-281. Budapest: Akademinai Kiado.

Akaike, H. (1974). A new look at the statistical model identification. IEEE Transactions on Automatic Control, 19, 6 , 716-723.

Anderson, T. W., \& Darling, D. A. (1952). Asymptotic theory of certain 'goodness of fit' criteria based on stochastic processes. The Annals of Mathematical Statistics, 23, 2, 193-212.

Arellano-Valle, R. B., Branco, M. D. \& Genton, M. G. (2006). A unified view on skewed distributions arising from selections. The Canadian Journal of Statistics , 34, 4, 581-601.

Aryal, G.R., Tsokos, C.P. (2009). On transmuted extreme value distribution with application. Nonliner Analysis, 71, e1401-e1407.

Atkinson, A. C. (1985). Plots, Transformations and Regression: An Introduction to Graphical Methods of Diagnostic Regression Analysis. New York: Oxford University Press.

Balakrishnan, N. \& Leung, M. Y. (1988) Orders statistics from the type i generalized logistic distribution Communications in Statistcs - Simulation and Computation, 17, 1, 25-50.

Barndorff-Nielsen, O.E. (1983). On a Formula for the Distribution of the Maximum Likelihood Estimator. Biometrika, 70, 343-365.

Barndorff-Nielsen, O.E. (1986). Inference on full or partial parameters, based on the standardized signed log likelihood ratio. Biometrika, 73, 307-322.

Barndorff-Nielsen, O. E., Blæsild, P., \& Eriksen, P. S. (1989). Decomposition and Invariance of Measures, and Statistical Transformation Models. Heidelberg:Springer.

Barndorff-Nielsen, O.E. (1991). Modified signed log likelihood ratio. Biometrika, 78, 557-563.

Barreto-Souza, W.,Vasconcellos, K.L.P. (2011). Bias and skewness in a general extreme-value regression model. Computational Statistics \& Data Analysis 55, 3, 1379-1393.

Beirlant, J., Goegebeur, Y., Segers, J., Teugels, J. (2004). Statistics of Extremes: Theory and Applications. UK Chichester: John Wiley \& Sons. 
Branko, M., Tsokos, C.P. (2009). Ordinary, Bayes, empirical Bayes, and non-parametric reliability analysis for the Gumbel failure model. Nonlinear Analysis: Theory, Methods \& Applications, 71, 12, e1426-e1436.

Bruxer, J., Thompson, A., \& Eng, P. (2008). St. Clair River Hydrodynamic Modelling Using RMA2 Phase 1 Report. Environment Canada.

Castillo, E.,Hadi, A. S., Balakrishnan, N. \& Sarabia, J. M. (2005). Extreme value and related models with applications in engeneering and science. New Jersey: John Wiley \& Sons.

Chan, P.S., Ng, H.K.T., Balakrishnan, N., Zhou, Q. (2008). Point and interval estimation for extreme-value regression model under type-II censoring. Computational Statistics and Data Analysis, 52, 4040-4058.

Coles, S. (2001). An Introduction to Statistical Modeling of Extremes. London: Springer-Verlag.

Cordeiro, G. M. (1987). On the corrections to the likelihood ratio statistics. Biometrika, 74, 265-74.

Cordeiro, G.M. \& Ferrari, S.L.P. (1991). A modified score test statistic having chi-squared distribution to order $n^{-1}$. Biometrika, 78, 573-582.

Cordeiro, G. M., Nadarajah, S., \& Ortega, E. M. (2012). The Kumaraswamy Gumbel distribution. Statistical Methods \& Applications, 21, 2, 139-168.

Cordeiro, G.M., Ortega, E.M.M. \& da Cunha, D.C.C. (2013). The Exponentiated Generalized Class of Distributions. Journal of Data Science, 11, 1-27.

Cordeiro, G.M., Paula, G.A. (1989). Improved likelihood ratio statistics for exponential family nonlinear models. Biometrika, 76, 93-100.

Cordeiro, G.M., Udo, M.C.T. (2008). Bias correction in generalized nonlinear models with dispersion covariates. Communications in Statistics. Theory and Methods, 37, 2219-2225.

Cox, D. R., \& Reid, N. (1987). Parameter orthogonality and approximate conditional inference. Journal of the Royal Statistical Society. Series B (Methodological) , 40, 1-39.

Cox, D.R. \& Reid, N. (1993). A note on the calculation of adjusted profile likelihood. Journal of the Royal Statistical Society. Series B, 55, 467-471.

Cribari-Neto, F. \& Ferrari, S.L.P. (1995). Second order asymptotics for score tests in generalised linear models. Biometrika, 82, 426-432.

Danielsson, J., Jorgensen, B. N., Sarma, M., \& de Vries, C. G. (2006). Comparing downside risk measures for heavy tailed distributions. Economics letters, 92(2), 202-208.

DiCiccio, T. J. (1984). On parameter transformations and interval estimation. Biometrika, 71, 3, 477-485.

DiCiccio, T. J. \& Martin, M. A., (1993). Simple modifications for signed roots of likelihood ratio statistics. Journal of the Royal Statistical Society, 55, 305-316.

Doornik, J. A. (2009). An Object-Oriented Matrix Language - Ox 6. London: Timberlake Consultants Press.

Dubey, S. D. (1969). A new derivation of the logistic distribution. Naval Research Logistics Quarterly, 16, 37-40. 
Faivre, R. \& Masle, J. (1988). Modeling potential growth of tillers in winter wheat. Acta Ecologica, 9, 179-196.

Ferrari, S.L.P. \& Cysneiros, A.H.M.A. (2008). Skovgaard's adjustment to likelihood ratio tests in exponential family nonlinear models. Statistics and Probability Letters, 78, 3047-3055

Ferrari, S.L.P. \& Pinheiro, E.C. (2011). Improved likelihood inference in beta regression. Journal of Statistical Computation and Simulation, 81, 4 , 431-443.

Ferreira da Silva, M., Ferrari, S.L.P. \& Cribari-Neto, F. (2008). Improved likelihood inference for the shape parameter in Weibull regression. Journal of Statistical Computation and Simulation, 78,9, 789-811.

Fraser, D.A.S., Reid, N. \& Wu, J. (1999). A simple general formula for tail probabilities for frequentist and bayesian inference. Biometrika, 86, 249-264.

Fréchet, M. (1927). Sur la loi de probabilité de l'écart maximum. Ann. Soc. Polon. Math Cracovie, 6, 32, 93-116.

Fisher, R. A. \& Tippett, L. H. C. (1928, April). Limiting forms of the frequency distribution of the largest or smallest member of a sample. In Mathematical Proceedings of the Cambridge Philosophical Society, 24, 2, 180-190. Cambridge University Press.

Gnedenko,B. (1943) Sur la distribuition limite du terme maximum d'une série aléatoire. Ann. Math., 44, 423-453. Breakthroughs in Statistics, Vol.I (1992), eds. S. Kotz and N.L. Johnson, Springer-Verlag, pp. 195-225. Traduzido e reimpresso em:

Gokarna, R.A. \& Tsokos, C.P. (2009) On the transmuted extreme value distribution with application. Nonlinear Analysis: Theory, Methods \& Applications, 71, 12, e1401-e1407

Gradshteyn, I.S. \& Ryzhik, I.M. (2000). Table os Integrals, Series and Products. Massachusetts : Academic Press.

Gumbel, E. J. (1935). Les valeurs extrêmes des distributions statistiques. Annales de l'Institut Henri Poincaré, 5, 2, 115-158. Presses universitaires de France.

Gumbel, E. J. (1937). Les intervalles extrêmes entre les émissions radioactives. II. J. Phys. Radium, 8, 11, 446-452.

Gumbel, E. J. (1937). La durée extrême de la vie humaine. Actualités Scientifique et Industrielles, 520. Hermann et cie.

Gumbel, E. J. (1941). The return period of flood flows. The Annals of Mathematical Statistics, 12, 2, 163-190.

Gumbel, E. J. (1944). On the plotting of flood discharges. Transactions American Geophysical Union, 24, 699-719.

Gumbel, E. J. (1945). Floods estimated by probability methods. Engineering News-Record, 134, 97-101.

Gumbel, E. J. (1948). The Statistical Forecast of Floods. Ohio Water Resources Board, 15, 1-21.

Gumbel, E.J. (1958). Statistics of Extremes. 2nd ed. New York: Columbia University Press.

Haan, L. de (1970). On Regular Variation and Its Application to the Weak Convergence of Sample Extremes. Mathematical Centre Tracts 32. Amsterdam: Mathematics Centre.

Hald, A. (1952). Statistical Theory with Engineering Applications. 7th ed. Canada:John Wiley \& Sons. 
Hand, D.J., Daly, F., Lunn, A.D., McConway, K.J. \& Ostrowsky, E. (1996) A Handbook of Small Data Sets. 2nd ed. London: Chapman \& Hall.

Huang, Guang-Hua. (2005). Model Identifiability Encyclopedia of Statistics in Behavioral Science, 3, 1249-1251. Chichester: John Wiley \& Sons. http://onlinelibrary.wiley.com/doi/10.1002/0470013192. bsa399/pdf.

Hubert, M. \& Vadervieren, E. (2008). An adjusted boxplot for skewed distributions. Computational Statistics \& Data Analysis, 52, 5186-5201.

Huet, S., Bouvier, A., Poursat, M.-A. \& Jolivet, E. (2004). Statistical Tools for Nonlinear Regression. 2nd ed. New York: Springer-Verlag.

Hurvich, C. M., \& Tsai, C. L. (1989). Regression and Time Series Model Selection in Small Samples. Biometrika, 76, 2, 297-307.

Hurvich, C. M., \& Tsai, C. L. (1995). Model selection for extended quasi-likelihood models in small samples. Biometrics, 51, 1077-1084.

Jenkinson, A. F. (1955). The frequency distribution of the annual maximum (or minimum) values of meteorological elements. Quarterly Journal of the Royal Meteorological Society, 81, 348, 158-171.

Jensen, J. L. (1986). Similar tests and the standardized log likelihood ratio statistic. Biometrika , 73, 3, 567-572.

Jensen, J. L. (1987). Standardized log-likelihood ratio statistics for mixtures of discrete and continuous observations. The Annals of Statistics, 314-324.

Kolmogorov, A. (1933). Sulla determinazione empirica di una legge di distribuzione. Giornale dell'Istituto Italiano degli Attuari , 4, 1-11.

Kotz, S. \& Nadajarah, S. (2000). Extreme Value Distributions : Theory and Applications. London: Imperial College Press.

Kotz, S. \& Vicari, D. (2005). Survey of developments in the theory of continuous skewed distributions. METRON International Journal of Statistics.

Kullback, S. (1959). Information Theory and Statistics. New York: Wiley.

Labeyrie, J. (1991). Time scales and statistical uncertainties in the prediction of extreme environmental conditions. Reliability Engineering \& System Safety, 32, 3, 243-266.

Lawley, D. (1956). A general method for approximating to the distribution of likelihood ratio criteria. Biometrika, 43, 295-303.

Lawless, J.F. (2003). Statistical Models and Methods of Lifetime Data. 2nd ed. New Jersey: John Wiley \& Sons.

Lehmann, E.L., Casella, E. (1998). Theory of Point Estimation. 2nd ed. New York: Springer-Verlag.

Lemonte, A.J. (2010). Estatística gradiente e refinamento de métodos assintóticos no modelo Birnbaum-Saunders. Tese de doutorado, IME - Universidade de São Paulo. 
Lemonte, A.J. \& Ferrari, S.L.P. (2011). Size and power properties of some tests in the Birnbaum-Saunders regression model. Computational Statistics and Data Analysis, 55, 1109-1117.

Lemonte, A.J. \& Ferrari, S.L.P. (2012). The local power of the gradient test. Annals of the Institute of Statistical Mathematics, 64, 2, 373-381.

Lien, J. W. (2011) Slot Machine Stopping Decisions: Evidence for Prospect Theory Preferences?

Luceño, A. (2005). Fitting the generalized Pareto distribution to data using maximum goodness-of-fit estimators. Computational Staatiscs \& Data Analysis, 51, 904-917.

Markovich, N. (2007). Nonparametric Analysis of Univariate Heavy-Tailed Data: Research and Practice Wiley Series in Probability and Statistics, 311-318.

McCullagh, P. (1984). Local sufficiency. Biometrika , 71, 2, 233-244.

Melo, T.F.N., \& Ferrari, S.L.P. (2010). A modified signed likelihood ratio test in elliptical structural models. AStA Advances in Statistical Analysis, 94, 1, 75-87.

von Mises, R. (1936). La distribution de la plus grande de $n$ valeurs. Rev. Math. Union Interbalcanique, 1, 141-160. Reproduzido em: Selected Papers of Richard von Mises., II, 271-294. Providence, RI.: American Mathematical Society.

Nadarajah, S. \& Kotz, S. (2004). The beta Gumbel distribution. Mathematical Problems in Engineering, 4, 323-332.

Nadarajah, S. (2006). The exponentiated Gumbel distribution with climate application. Environmetrics, 17, 13-23.

Nelder, J.A. \& Wedderburn, R.W.M. (1972). Generalized linear models. Journal of the Royal Statistical Society A, 135, 370-384.

Nocedal, J. \& Wright, S.J. (1999). Numerical Optimization. New York: Springer-Verlag.

Ojo, M. O. (2001). Some relationships between the generalized Gumbel and other distributions. Kragujevac J. Math., 23, 101-106.

Paula, G.A., Rojas O.V. (1997). On restricted hypotheses in extreme value regression models. Computational Statistics \& Data Analysis, 25, 2, 143-157.

Pescim, R. R.,Cordeiro, G. M., Demétrio, C. G. B., Ortega, E. M., Nadarajah, S. (2012). The new class of Kummer beta generalized distributions. SORT-Statistics and Operations Research Transactions, 36(2), 153-180.

Prentice,R.L. (1975). Discrimination Among Some Parametric Models. Biometrika, 62, 3, 607-614.

Prentice,R.L. (1976). A Generalization of the Probit and Logit Methods for Dose Response Curves. Biometricsa, 32, 4, 761-768.

Press, W.H., Teulosky, S.A., Vetterling, W.T. \& Flannery, B.P, (1992). Numerical Recipes in C: The Art of Scientific Computing. 2nd ed. London: Prentice Hall .

R Core Team (2013). R: A Language and Environment for Statistical Computing. The R Foundation for Statistical Computing, Vienna, Austria. http://www.R-project.org. 
Resnick, S. (2007). Heavy-Tail Phenomena: Probabilistic and Statistical Modeling. Springer.

Ripley, B. D. (1977). Modelling spatial patterns (with discussion). Journal of the Royal Statistical Society-Series B,39, 172-212.

Ross, S. (2002). A First Course in Probability. 6th ed. New Jersey : Prentice Hall.

Rossi, F., Fiorentino, M., \& Versace, P. (1984). Two-component extreme value distribution for flood frequency analysis. Water Resources Research, 20, 7, 847-856.

Sanford, L. P. (1997). Turbulent mixing in experimental ecosystem studies. Marine Ecology Progress Series, 161, 265-293.

Shaw, W. T., \& Buckley, I. R. (2007). The Alchemy of Probability Distributions: Beyond Gram-Charlier \& CornishFisher Expansions, and Skew-Normal or Kurtotic-Normal Distributions. Submitted, Feb, 7.

Severini, T.A. (1999). An empirical adjustment to the likelihood ratio statistic. Biometrika, 86, 235-247.

Severini, T.A. (2000). Likelihood Methods in Statistics. Oxford: Oxford University Press.

Skovgaard, I.M. (1996). An explicit large-deviance approximation to one-parameter tests. Bernoulli, 2, 145-165.

Skovgaard, I.M. (2001). Likelihood asymptotics. Scandinavian Journal of Statistics, 28, 3-32.

Smirnov, N. (1939). On the estimation of the discrepancy between empirical curves of distribution for two independent samples. Bulletin Mathématique de l'Université de Moscou, 2, 2.

Smith, R.L. \& Naylor, J.C. (1987). A comparison of maximum likelihood and Bayesian estimators for the threeparameter Weibull distribution. Applied Statistics, 36, 358-369.

Schwarz, G. (1978). Estimating the Dimension of a Model. The Annals of Statistics, 6, 2, 461-464.

Terrell, G. R. (2002). The gradient statistic. Computing Science and Statistics, 34, 206-215.

Wei, B.C. (1998). Exponential Family Nonlinear Models. Singapore: Springer-Verlag.

Weibull, W (1939). A Statistical Theory of the Strength of Material. Ingeniörs Vetenskaps Akademiens Handlingar, 151.

Wolfram Research, Inc. (2012) Mathematica Edition: Version 9.0. Champaign, Illinois: Wolfram Research, Inc.

Wu, J., \& Jiang, G. (2007). Confidence intervals of effect size for paired studies. Biometrical Journal , 49, 5, 765-773.

Wu, J., Jiang, G., \& Wei, W. (2006). Confidence intervals of effect size in randomized comparative parallel-group studies. Statistics in medicine , 25, 4, 639-651.

Wu, J. \& Wong, A. C. (2004). Improved interval estimation for the two-parameter Birnbaum-Saunders distribution. Computational Statistics \& Data Analysis , 47, 4, 809-821.

Wu, J., Wong, A. C. M. \& Wei, W. (2006). Interval estimation of the mean response in a log-regression model. Statistics in medicine, 25, 12, 2125-2135. 